\title{
Triggered ventricular arrhythmias in the hypertrophied heart : the role of electrophysiological and functional adaptations
}

Citation for published version (APA):

de Groot, S. H. M. A. (1997). Triggered ventricular arrhythmias in the hypertrophied heart : the role of electrophysiological and functional adaptations. [Doctoral Thesis, Maastricht University]. Universiteit Maastricht. https://doi.org/10.26481/dis.19980123sg

Document status and date:

Published: 01/01/1997

DOI:

10.26481/dis.19980123sg

Document Version:

Publisher's PDF, also known as Version of record

Please check the document version of this publication:

- A submitted manuscript is the version of the article upon submission and before peer-review. There can be important differences between the submitted version and the official published version of record.

People interested in the research are advised to contact the author for the final version of the publication, or visit the DOI to the publisher's website.

- The final author version and the galley proof are versions of the publication after peer review.

- The final published version features the final layout of the paper including the volume, issue and page numbers.

Link to publication

\footnotetext{
General rights rights.

- You may freely distribute the URL identifying the publication in the public portal. please follow below link for the End User Agreement:

www.umlib.nl/taverne-license

Take down policy

If you believe that this document breaches copyright please contact us at:

repository@maastrichtuniversity.nl

providing details and we will investigate your claim.
}

Copyright and moral rights for the publications made accessible in the public portal are retained by the authors and/or other copyright owners and it is a condition of accessing publications that users recognise and abide by the legal requirements associated with these

- Users may download and print one copy of any publication from the public portal for the purpose of private study or research.

- You may not further distribute the material or use it for any profit-making activity or commercial gain

If the publication is distributed under the terms of Article $25 \mathrm{fa}$ of the Dutch Copyright Act, indicated by the "Taverne" license above, 


\section{Triggered ventricular arrhythmias in the hypertrophied heart}

The role of electrophysiological and functional adaptations

\section{PROEFSCHRIFT}

ter verkrijging van de graad van doctor aan de Universiteit Maastricht, op gezag van de Rector Magnificus, Prof. dr. A.C. Nieuwenhuijzen Kruseman, volgens het besluit van het College van Decanen, in het openbaar te verdedigen op vrijdag 23 januari 1998 om 12.00 uur

door

Sofia Hendrika Maria Alberta de Groot geboren te Boxmeer 
ISBN 90-5681-025-1.

(C) S.H.M.A. de Groot, Maastricht 1997 Omslag: Claudia Volders Lay-out: Harmke de Groot

Druk: Unigraphic, Universiteit Maastricht 


\section{Promotor:}

Prof. dr. H.J.J. Wellens

\section{Co-promotor:}

Dr. M.A. Vos

\section{Beoordelingscommissie:}

Prof. dr. H.A.J. Struijker Boudier (voorzitter)

Prof. dr. M.A. Allessie

Prof. dr. E. Carmeliet (CARIM; Katholieke Universiteit Leuven, Leuven, België)

Prof. dr. H.E.D.J. ter Keurs (University of Calgary, Calgary, Canada)

Prof. dr. M.R. Rosen (Columbia University, New York, U.S.A.)

De uitgave van dit proefschrift werd mede mogelijk gemaakt door financiële steun van de Stichting RESCAR te Maastricht.

Financiële steun werd verder onder andere geleverd door: BARD-Electrophysiology, Byk Nederland, Pharmacia \& Upjohn, Roche Pharmaceuticals 
Met dank aan mijn ouders Voor Maurits 



\section{Contents}

\section{Chapter 1}

Arrhythmogenesis in (de)compensated cardiac hypertrophy. Review of the literature and introduction to the thesis

\section{Chapter 2}

Combining monophasic action potential recordings with pacing to demonstrate delayed afterdepolarizations and triggered arrhythmias in the intact heart: Value of the diastolic slope

\section{Chapter 3}

The dynamic behavior of the diastolic slope of the monophasic action potential can be related to the occurrence and maintenance of delayed afterdepolarization dependent arrhythmias in the canine heart

51

\section{Chapter 4}

Dualistic effects of ryanodine on ouabain induced delayed afterdepolarization dependent ventricular tachycardia

73

\section{Chapter 5}

Enhanced susceptibility for acquired Torsade de Pointes arrhythmias in the dog with chronic, complete atrio-ventricular-block is related to cardiac hypertrophy and electrical remodeling

95

\section{Chapter 6}

Adaptations to preserve cardiac function predispose the hypertrophied canine heart for delayed afterdepolarization dependent arrhythmias 


\section{Chapter 7}

Hypertrophy in the canine heart leads to an attenuated force-frequency relation with no signs of left or right ventricular pump failure

\section{Chapter 8}

General discussion

Summary

Samenvatting

Dankwoord 205

Publications 209

Curriculum Vitae 213 


\section{Chapter 1}

\section{Arrhythmogenesis in (de)compensated cardiac hypertrophy \\ Review of the literature and introduction to the thesis}




\subsection{Clinical background}

\section{Ventricular arrhythmias and sudden death in congestive heart failure}

During the past decades, congestive heart failure has become one of the most important health problems in cardiovascular medicine. As more people with ischemic ${ }^{\mathbb{1}}$ or nonischemic heart disease are saved from a premature death, more patients will develop heart failure. ${ }^{2}$ Additionally, aging of the population has contributed to the increased incidence of heart failure. In their fifties, only $1 \%$ of persons are affected by heart failure, while this number increases to $10 \%$ of persons in their eighties. ${ }^{2}$ Consequently, the number of hospitalizations for heart failure is increasing. In The Netherlands, the hospital admittance rate for heart failure increased by $69 \%$ in the period 1980-1992, and the cost of health care for patients suffering from heart failure was estimated to be $1 \%$ of the total cost of national health care. ${ }^{3}$

Heart failure has a bad prognosis, with mortality being most often caused by 1) sudden arrhythmic death and 2) death resulting from progressive pump failure. ${ }^{2,4,5}$ The relation between total one year mortality and sudden death according to NYHA (New York Heart Association) functional class is illustrated in figure 1 (adapted from references ${ }^{4,6-10}$ ). Although total mortality is low among patients with mild to moderate heart failure (class I and II), the proportion of patients dying suddenly is large; $50-70 \%$ of all death are sudden in class I and II. $4,7,10$ Patients in class III and IV have a worse overall prognosis with a mortality of $40-60 \%$. However, in these classes, only $30-40 \%$ of total deaths seems to be caused by sudden death, ${ }^{6-9}$ because mortality is caused by progressive deterioration of myocardial function. ${ }^{5}$

Since ventricular hypertrophy accompanies or precedes heart failure in $75 \%$ of patients ${ }^{5}$ and because of the high incidence of sudden cardiac death in patients with a reasonable functional class, attention has been shifted to cardiac hypertrophy itself as a possible predisposing factor for ventricular arrhythmias and sudden arrhythmic death.

\section{Ventricular arrhythmias and sudden death in compensated cardiac hypertrophy}

While congestive heart failure represents a decompensated or deadapted state, cardiac hypertrophy is an adaptive mechanism of the heart to mechanical overload of any cause. The prevalence of left ventricular hypertrophy in the total adult population, diagnosed by ECG or echocardiography, is $10-20 \%$ and increases with age. ${ }^{11}$ Among men and women under 30 years the incidence of echocardiographic left ventricular hypertrophy 


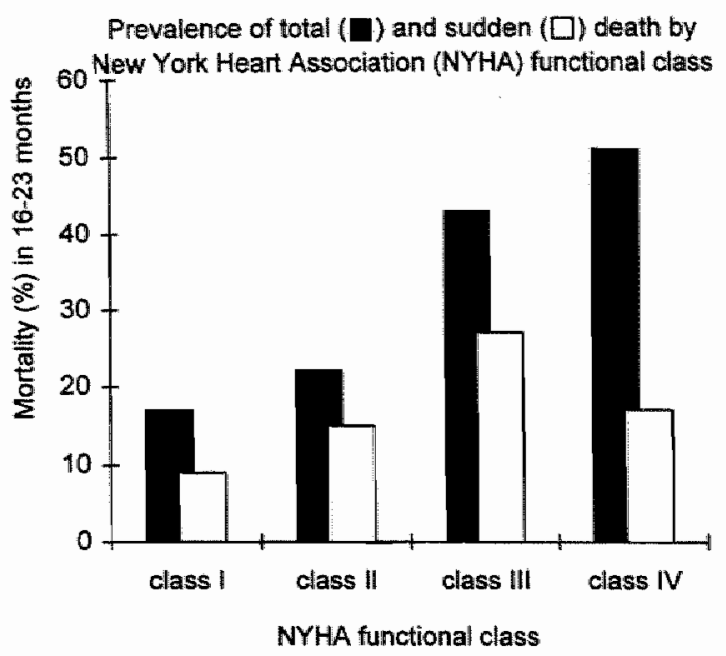

Figure 1: Prevalence of total and sudden death by New York Heart Association (NYHA) functional class and 16 to 23 month mortality, adapted from different references $(4,6-10)$.

is $8 \%$ and $5 \%$ respectively, whereas after the age of 70 , these values increase to $33 \%$ and $49 \% .^{12}$

Left ventricular hypertrophy has been associated with 1) an increased susceptibility to ventricular arrhythmias ${ }^{13-16}$ and 2) an increased risk of sudden arthythmic death. ${ }^{17,18}$ The risk to develop lethal arrhythmias in hypertrophied hearts seems to exceed the risk attributable to coexisting factors, like ischemia ${ }^{18}$ or decompensation ${ }^{16,17}$ However, the exact relation between ventricular arrhythmias, sudden death and functional status has not been studied properly.

Many studies have suggested a relation between the incidence and severity of ventricular arrhythmias registered with Holter and the severity (NYHA class) of heart failure. ${ }^{19,20}$ While there exists a positive relation between the Holter data and total mortality, this relation is not present for Holter and sudden death, indicating additional factors. ${ }^{21}$ Additionally, in most studies on ventricular arrhythmias, information regarding cardiac function is lacking. It is difficult to discriminate between compensated and decompensated hypertrophy, or between the transition from hypertrophy to heart failure. Especially in humans, the determination of the disease state is complicated, because of concomitant 
Table 1: Overview of the canine models used to induce cardiac hypertrophy and/or heart failure, according to experimental technique

\section{Experimental technique}

CHF

Hypertrophy

References

1) Pressure loading

$\begin{array}{llll}\text { Aortic banding } & \mathrm{N} / \mathrm{Y}^{*} & \mathrm{LV} & 24-27,31 \\ \text { Experimental hypertension } & \mathrm{N} & \mathrm{LV} & 28,30 \\ \text { Pulmonary artery banding } & \mathrm{N} & \mathrm{RV} & 29\end{array}$

2) Volume loading

$\begin{array}{llll}\text { Mitral regurgitation } & \mathrm{Y} & \mathrm{LV} & 31,32 \\ \text { Tricuspid regurgitation } & \mathrm{Y} & \mathrm{RV} & 33 \\ \text { Aorto-to-vena cava fistula } & \mathrm{N} & \mathrm{LV} / \mathrm{RV} & 34,35,51 \\ \text { Complete heart block } & \mathrm{N} / \mathrm{Y}^{*} & \mathrm{LV} / \mathrm{RV} & 36-41\end{array}$

3) Rapid pacing

4) Myocardial ischemia/infarction

Coronary micro-embalization DC-shock

$\begin{array}{ccc}\text { Y } & \text { LV } & 46,47 \\ N & \text { LV } & 48,49\end{array}$

5) Intoxications

Norepinephrine

* depending on reference LV 50

CHF: congestive heart failure, $L V$ : left ventricle, $R V$ : right ventricle; $Y$ : present; $N$ : not present 
pharmacological treatment leading to regression of heart failure symptoms. Furthermore, cardiac function can be abnormal in response to stress (exercise), while normal during baseline (rest) circumstances. ${ }^{22,23}$ Studies that tested the response of the hypertrophied heart to increasing demands by increasing the heart rate with pacing or by studying post-extrasystolic potentiation, ${ }^{22,23}$ have not related their observations to susceptibility to (lethal) arrhythmias.

Insight in the different disease stages could be important to develop appropriate therapies and to determine the timing of the interventions. To unravel the complex nature of the disease process, animal models of hypertrophy with or without subsequent development of heart failure could provide useful alternatives to clinical studies.

\subsection{Canine models to study (de)adaptations in cardiac hypertrophy}

This overview is limited to the description of adult dog models leading to 1) hypertrophy, 2) hypertrophy with heart failure symptoms, and 3) heart failure without hypertrophy. This limitation is made because of the applicability of clinically used techniques in large animals. In addition the electrophysiology of the canine heart has been extensively described.

\section{Characteristics of the dog models}

At present, several approaches have been used to induce hypertrophy and/or heart failure with involvement of the left, the right or both ventricles. These include: 1) pressure loading, $24-31$ 2) volume loading, , $^{31-41,51}$ 3) rapid pacing, ${ }^{35,42-45}$ 4) myocardial necrosis, $^{46-49}$ and 5) intoxications ${ }^{50}$ (table 1). Not all different approaches are equally effective in inducing hypertrophy and/or heart failure (tables 1 and 2).

While heart failure symptoms, like pulmonary congestion, are induced by rapid pacing, intracoronary micro-embolization, mitral regurgitation and tricuspid regurgitation, they are less inducible when hypertension, aorto-to-vena cava fistula, norepinephrine infusion and DC-shock are applied (table 1). Aortic bandage only induces heart failure in a sub-population of dogs, especially in those with the most severely hypertrophied hearts. ${ }^{24,26}$ In our model, the dog with complete atrioventricular-block, both the presence $^{37}$ and the absence ${ }^{36,38,40,41}$ of heart failure symptoms have been described. Similarly, not all models are equally effective in inducing hypertrophy; both the presence 
Table 2: Amount of left and/or right ventricular hypertrophy induced in the different cantine modes

Experimental technique

Duration

Weight increase

References

LV RV

1) Pressure loading

\begin{tabular}{|c|c|c|c|}
\hline Aontic banding & 3 months & $+86 \%$ & \\
\hline Aortic banding, moderate & 10-12 months & $+47 \%$ & $=$ \\
\hline Aortic banding, severe & 11-13 months & $+87 \%$ & $=$ \\
\hline Aortic banding & $1-1.5$ years & $+63 \%$ & $=$ \\
\hline Experimental hypertension & $1-12$ weeks & $+29 \%$ & \\
\hline Experimental hypertension & $7-9$ weeks & $+36 \%$ & \\
\hline Pulmonary artery banding & 2.40 weeks & $+29 \%$ & $+100 \%$ \\
\hline
\end{tabular}

2) Volume loading

$\begin{array}{lllll}\text { Aorto-to-vena cava shunt } & 324 \text { days } & +40 \% & +68 \% & 51 \\ \text { Aorto-to-vena cava shunt } & 3-4 \text { weeks } & +22 \% & +12 \% & 106 \\ \text { Mitral regurgitation } & 3 \text { months } & +25 \% & & 31 \\ \text { Mitral regurgitation } & 3 \text { months } & +21 \% & & 32 \\ \text { atrioventricular block } & 4-36 \text { months } & +23 \% & +44 \% & 38 \\ \text { atrioventricular block } & 2 \text { weeks } & +18 \% & +35 \% & 41 \\ & 10 \text { weeks } & +21 \% & +71 \% & 41\end{array}$

3) Rapid pacing

Rapid pacing

4 weeks

$=$

Rapid pacing

24 days

$=$

4) Intoxitions

Norepinephrine

6-63 weeks

$+14 \%$

$=$

50

5) Miscllneous

Tricuspid regurgitation \& 8 weeks

$=$

$+50 \%$ 33 pulmonary artery banding = means no change, $L V$ : left ventricle, $R V$ : right ventricle 
or the absence and the amount of hypertrophy differ considerably. Also, the time periods used to characterize the models are variable (table 2). The largest increase in left ventricular mass seems to result from pressure loading of the left ventricle, with aortic banding resulting in more weight increase than hypertension (table 2). ${ }^{24,26-28,30}$ Volume loading results in a $20-25 \%$ increase in left ventricular mass, more or less independent of the method used (table 2). ${ }^{31,32,38,40,41}$ Besides inducing left ventricular hypertrophy, volume loading can also induce right ventricular hypertrophy (table 2). ${ }^{38,40,41}$ In contrast, in the rapid-pacing model, heart failure occurs without any preceding or accompanying hypertrophy. $35,42-45$

In most models, several hemodynamic, neurohumoral and structural adaptations have been found (table 3 ). The pacing induced heart failure model and the repetitive microembolization heart failure model are the most extensively described models, ${ }^{35,42-47}$ while limited information is available about the adaptation process of the dogs with experimental hypertension (table 3 ). ${ }^{28,30}$ Repetitive coronary micro-embolization results in a sustained depression of left ventricular function and a dilated hypertrophied left ventricle. ${ }^{46,47}$ After discontinuation of micro-embolization, heart failure sustains. ${ }^{46,47}$ The development of heart failure in this model is accompanied by large increases in plasma levels of neurohumoral parameters. At histological examination, increased collagen volume and decreased capillary density are found (table 3 ). ${ }^{46}$ In contrast, the collagen volume ${ }^{35}$ and capillary density, ${ }^{51}$ in the aorto-caval fistula model of volume overload induced hypertrophy remain normal, clearly illustrating the existence of different adaptation processes in the different models.

\section{Sensitivity to ventricular arrhythmias and sudden arrhythmic death}

Of the described canine models of hypertrophy and/or heart failure, none has been developed specifically to study the presence or absence of an increased susceptibility to (lethal) arrhythmias. However, in a few studies a relation has been sought between the pathological conditions and the propensity to develop arrhythmias.

Using Holter recordings, a high incidence of spontaneously occurring arrhythmias was found in the dog with congestive heart failure, independent whether it was caused by rapid pacing or by repetitive micro-embolizations ${ }^{52,53}$ Besides the spontaneous occurrence of arrhythmias, also the inducibility of arrhythmias by programmed electrical stimulation was tested. In both models, programmed electrical stimulation resulted 
Table 3: Hemodynamic, structural and neurohumorall characterization of the canine models used to induce left wentricular hypentrophy and or heart failure

\begin{tabular}{|c|c|c|c|}
\hline $\begin{array}{l}\text { Method } \\
\text { Duration } \\
\text { References }\end{array}$ & $\begin{array}{l}\text { Aortic bandage } \\
(1-1.5 \text { years })\end{array}$ & $\begin{array}{l}\text { Hypertension } \\
(7-9 \text { weeks) } \\
28 ; 30\end{array}$ & $\begin{array}{l}\text { AV-shumt } \\
(4-10 \text { weeks) } \\
34,35,51,107\end{array}$ \\
\hline Heart rate & $=$ & $\uparrow$ & $T T$ \\
\hline \multicolumn{4}{|l|}{ Hemodynamics } \\
\hline $\begin{array}{l}\angle V E F \\
C O / C I\end{array}$ & & & $\uparrow \uparrow$ \\
\hline$\angle V E D P$ & $\uparrow$ & & $\uparrow \uparrow \uparrow$ \\
\hline LVESP & $\uparrow \uparrow$ & $\uparrow \uparrow$ & $=$ \\
\hline$+L V d P / d t$ & $=$ & & \\
\hline \multicolumn{4}{|l|}{$L V d P i d t$} \\
\hline LVEDV/LVEDD & $\Rightarrow$ & & $\uparrow$ \\
\hline \multicolumn{4}{|l|}{ RVESP } \\
\hline \multicolumn{4}{|l|}{ RVEDP } \\
\hline \multicolumn{4}{|l|}{$+R V d P / d t$} \\
\hline \multicolumn{4}{|c|}{ Neurohumoral (plasma) } \\
\hline$N E$ & & & \\
\hline$A N F$ & $\uparrow$ & & \\
\hline \multicolumn{4}{|l|}{ Renin actiwity } \\
\hline \multicolumn{4}{|l|}{ Angiotenstin $I I$} \\
\hline \multirow{2}{*}{\multicolumn{4}{|c|}{ Structural }} \\
\hline & & & \\
\hline$H / B W$ & $\uparrow \uparrow$ & Mp & $\uparrow$ \\
\hline$\angle W / B W$ & $\uparrow \uparrow$ & $\uparrow$ & $\uparrow$ \\
\hline$R W / B W$ & $=$ & & $=$ \\
\hline LVWT & & & $=$ \\
\hline \multicolumn{4}{|l|}{ RVWT } \\
\hline Capillary density & & & $=$ \\
\hline Collagen & & & $=$ \\
\hline
\end{tabular}

LVEF: left ventricular ejection fraction, $\mathrm{CO} / \mathrm{CI}$ : cardiac output or cardiac index, LVEDP: left ventricular end diastolic pressure, LVESP: left ventricular end systolic pressure, LVEDV/LVEDD: left ventricular end diastolic volume or enddiastolic diameter, LVESV: left ventricular end systolic volume, RVESP: right wentricular end systolic pressure. 
Table 3 (continued)

\begin{tabular}{|c|c|c|c|c|}
\hline $\begin{array}{l}\text { AV-block } \\
\text { (2 weeks-3 years) } \\
38,40,41\end{array}$ & $\begin{array}{l}\text { Mitral regurgitation } \\
(1-3 \text { months }) \\
25,32\end{array}$ & $\begin{array}{l}\text { Micro-embolization } \\
\text { (13 weeks) } \\
46,47\end{array}$ & $\begin{array}{l}\text { DC-shock } \\
\text { (4 months) } \\
48,49\end{array}$ & $\begin{array}{l}\text { Rapid pacing } \\
(3-5 \text { weeks) } \\
35,42-44\end{array}$ \\
\hline$\downarrow$ & $\uparrow$ & $\pi$ & $=$ & $\uparrow \uparrow$ \\
\hline$\uparrow$ & & $\downarrow \downarrow$ & $\downarrow$ & \\
\hline$=$ & $\uparrow \uparrow \uparrow$ & $\downarrow$ & $\downarrow \downarrow$ & $\downarrow \downarrow$ \\
\hline$=$ & $\uparrow \uparrow \uparrow$ & $\uparrow \uparrow \uparrow$ & & $\uparrow \uparrow \uparrow$ \\
\hline$\uparrow$ & - & $\downarrow$ & & $\downarrow \downarrow$ \\
\hline$=$ & & $\downarrow$ & & \\
\hline \multirow[t]{4}{*}{$\uparrow$} & $\uparrow \uparrow(\uparrow)$ & $\uparrow$ & $\uparrow$ & $\begin{array}{l}\uparrow \uparrow \\
\uparrow \uparrow \\
\uparrow \uparrow \uparrow \\
\downarrow \downarrow\end{array}$ \\
\hline & & $\begin{array}{l}\uparrow \uparrow \uparrow \\
\uparrow \uparrow \uparrow\end{array}$ & $\uparrow \uparrow$ & $\begin{array}{l}\uparrow \uparrow \uparrow \\
\uparrow \uparrow \uparrow\end{array}$ \\
\hline & & $\uparrow$ & $=$ & $\uparrow \uparrow \uparrow$ \\
\hline & & & & $\uparrow \uparrow \uparrow$ \\
\hline$\uparrow$ & & $\uparrow$ & $\uparrow$ & $=$ \\
\hline$\uparrow$ & $\uparrow$ & & $\uparrow$ & $=$ \\
\hline$\uparrow \uparrow$ & $=$ & & $=$ & $=$ \\
\hline$\uparrow$ & & $\uparrow$ & & $=$ \\
\hline & $=$ & $\uparrow \uparrow$ & & $\uparrow \uparrow$ \\
\hline
\end{tabular}

RVEDP: right ventricular end diastolic pressure, NE: norepinephrine, ANF: atrial natriuretic factor, H/BW: heart to bodyweight ratio, LV/BW: left ventricle to bodyweight ratio, $\mathrm{RV} / \mathrm{BW}$ : right ventricle to body weight ratio, LVWT: left ventricular wall tickness, RVWT: right ventricular wall tickness, $\uparrow$ increased, $\downarrow$ decreased, $=$ no change . 
frequently in the induction of ventricular fibrillation in dogs with heart failure in contrast to their non-failing controls, either with or without catecholamine infusion. ${ }^{53-55}$ Additionally, the hypertrophied hearts show an increased propensity to develop ventricular tachycardia, ventricular fibrillation and sudden death during myocardial ischemia. $^{28,56,57}$

In ventricular hypertrophy and heart failure, prolongation of repolarization is the most consistently found electrophysiological change. This has not only been reported in the above described models, ${ }^{30,51,58}$ but also in other animal models of (de)compensated hypertrophy ${ }^{59,60}$ and in humans with congestive heart failure. ${ }^{61}$ It has been suggested that these repolarization disturbances predispose the heart for early afterdepolarizations, related ectopic beats and Torsade de Pointes arrhythmias, polymorphic ventricular tachycardias and sudden arrhythmic death.

\subsection{Mechanisms of arrhythmias in (de)compensated cardiac hypertrophy}

\section{Mechanisms of ventricular arrhythmias}

Although the increased susceptibility of the hypertrophied and failing hearts to ventricular arrhythmias has been extensively described, the pathophysiology of these arrhythmias is not well understood, with the exception of the lengthening of repolarization. In general, cardiac arrhythmias can be based on 1) abnormalities in impulse conduction (i.e. reentry), 2) abnormalities in impulse generation (i.e. abnormal automaticity, triggered activity) or 3) a combination of both. ${ }^{62}$ Impulse generation principally involves a cellular (focal) arrhythmogenic mechanism, while impulse conduction is based on the communication between cells and structures.

\section{Reentry}

Under normal conditions, impulse propagation over the ventricle is homogenous and the refractory periods are sufficiently long to prevent re-excitation so that a propagated impulse dies out. When a propagated impulse is able to recycle or "reenter" a conduction pathway, part or all of the heart can be re-excited producing a continuous arrhythmia. ${ }^{62-}$ 65 This reentry arrhythmia requires: 1) at least two alternative pathways for impulse conduction, 2) unidirectional or transient conduction block in one pathway, and 3) slowed conduction so that conduction time in the reentry circuit exceeds the refractory period of the conducting tissue. Thus, reentry is favored by conditions that slow conduction, 
shorten the refractory period, or cause differences in various parts of the reentry circuit. Different types of reentrant excitation can be distinguished: 1) circus movement reentry, using an anatomical substrate, ${ }^{65,66}$ 2) leading circle reentry, ${ }^{64} 3$ ) anisotropic reentry, ${ }^{63}$ and 4) combinations of these types. In general, ventricular arrhythmias due to reentry can be reproducibly induced and terminated by electrical stimulation. ${ }^{66,67}$

\section{Automaticity}

Automaticity is the ability of cells to initiate spontaneous action potentials arising as a result of phase 4 depolarization. Automaticity is a normal phenomenon in cells of the sinus node and the conducting system. "Normal automaticity" is automaticity arising from cells with a normal negative resting membrane potential, in contrast to "abnormal automaticity" that arises from a reduced diastolic potential in cardiac celis that normally show a high diastolic potential (i.e. $\pm-90 \mathrm{mV}$ ) ${ }^{62}$ Arrhythmias due to abnormal automaticity are not inducible by electrical stimulation, but occur spontaneously. ${ }^{62}$ For termination of these arrhythmias, single extra-stimuli will not be effective. The response to overdrive pacing depends on the membrane potential from which the abnormal rhythm arises: from about -90 through $-50 \mathrm{mV}$ progressively less and less overdrive suppression results in response to a constant period of pacing. ${ }^{68}$ Following termination these arrhythmias will reoccur spontaneously. ${ }^{68}$ In vivo, ventricular tachycardias based on abnormal automaticity have been described to occur spontaneously 16-24 hours after occlusion of the left anterior descending artery. ${ }^{69}$

\section{Triggered activity}

Triggered activity is abnormal impulse generation caused by afterdepolarizations occurring during repolarization (early afterdepolarization), or after repolarization is completed (delayed afterdepolarization). ${ }^{70}$ These afterdepolarizations are depolarizations of the membrane potential during or after an action potential and their occurrence depends on that action potential. ${ }^{70}$ When these depolarizations are large enough to reach threshold levels, a new action potential or series of action potentials can be initiated. ${ }^{70}$ With programmed stimulation both delayed and early afterdepolarization-dependent arrhythmias can be induced. ${ }^{67,70-73}$

Delayed afterdepolarizations have been associated with intracellular calcium overload and spontaneous calcium release from the sarcoplasmic reticulum into the cytosol. This sarcoplasmic reticulum calcium release generates an aftercontraction and a transient 
inward current that is responsible for delayed afterdepolarizations. ${ }^{70,74}$ Proposed mechanisms underlying the transient inward current are a calcium activated nonspecific channel, a cloride current and the $\mathrm{Na}^{+} / \mathrm{Ca}^{2+}$-exchanger. ${ }^{75-77}$ Less consensus exists regarding the ionic mechanism underlying early afterdepolarizations. In part, this is caused by the variety of circumstances during which early afterdepolarizations can be generated, suggesting that there is not one type of early afterdepolarization. Several sarcolemma-dependent mechanisms have been implicated as the currents underlying early afterdepolarizations, including the $\mathrm{L}$ - and $\mathrm{T}$-type calcium current as well as a sodium current. ${ }^{78}$ Additionally, during beta-adrenergic receptor stimulation, a sarcoplasmic reticulum dependent mechanism has been suggested to underlie the early afterdepolarizations. ${ }^{79-81}$ In this situation, both delayed and early afterdepolarizations can be induced. $^{79-81}$

\section{Arrhythmia promoting factors in (de)compensated hypertrophy}

Since (de)compensated hypertrophy is a multi-factorial disease, several factors may predispose for the occurrence of ventricular arrhythmias. These factors can relate to 1) structural and cellular abnormalities, 2) neurohumoral abnormalities, 3) electrolyte disturbances and 4) pharmacological treatment (table 4). ${ }^{82}$ The relation of the different factors with arrhythmogenesis is complex and dynamic, especially since their importance varies in each individual situation. Additionally, some of these factors can provoke regional differences in response.

\section{Structural abnormalities}

Structural abnormalities such as scar tissue or fibrosis, provide the anatomical substrate for reentry. ${ }^{83}$ Another structural adaptation that is not necessarily homogeneous in nature, is of course hypertrophy itself. Non homogeneous hypertrophy can cause non homogeneous lengthening of cardiac repolarization (cellular adaptation, see below), which may facilitate the occurrence of reentrant arrhythmias. ${ }^{92}$

Ventricular dilatation and ischemia can result in an increased myocardial heterogeneity of myocardial repolarization and decreased myocardial refractoriness, favoring the occurrence of a reentry mechanism ${ }^{84-87}$ However, ventricular dilatation and ischemia can also favor the occurrence of triggered activity. ${ }^{84,88-91}$ Additionally, ischemia can cause abnormal automaticity. 


\section{Cellular abnormalities}

At the cellular level, prolongation of the action potential duration is the most consisting finding in cardiac hypertrophy and failure..$^{30,58-61,93}$ This prolonged duration of repolarization can predispose for the development of early afterdepolarizations that are large enough to propagate and thus evoke arrhythmias. 30,60

Additionally, in hypertrophy and heart failure, calcium handling is abnormal. ${ }^{43,93-96}$ Besides resulting in an abnormal contractile function, this could result in an intracellular calcium overload. ${ }^{95}$ Intracellular calcium overload has been associated with delayed afterdepolarization-dependent triggered activity. As stated previously, still some controversy exists with respect to the occurrence of early afterdepolarization-dependent triggered activity during calcium overload. ${ }^{79-81}$

\section{Neurohumoral and electrolyte abnormalities}

In heart failure, the level of circulating catecholamines is persistently elevated. $^{35,42,44,46,49,82,97}$ Increased circulating catecholamines can facilitate the occurrence of all of the arrhythmogenic mechanisms. Additionally, they can increase myocardial oxygen demand and increase the tendency to hypokalemia. ${ }^{85}$ The occurrence of triggered activity is promoted by hypokalemia and hypomagnesemia. ${ }^{60,98}$

\section{Pharmacological treatment}

Many of the drugs used in the treatment of patients with heart failure, may provoke arrhythmias. By promoting electrolyte disturbances, diuretics can promote arrhythmias. Positive inotropic agents, including digitalis, increase both cardiac contractility and irritability of the ventricles. Digitalis promotes the occurrence of delayed afterdepolarizations by inducing intracellular calcium loading. ${ }^{99}$ In the presence of hypokalemia, arrhythmias may occur even at therapeutic levels of digitalis. ${ }^{100}$ Treatment with class Ic anti-arrhythmic agents can result in reentrant arrhythmias, because of the rate dependent slowing of conduction, ${ }^{101}$ while anti-arrhythmic agents that prolong action potential duration can promote the occurrence of early afterdepolarizations. $30,60,71,72$

In summary, the increased propensity of the hypertrophied and failing hearts to ventricular arrhythmias has been extensively described. Regarding the involved arrhythmogenic mechanism, no uniformity exists. Especially in the intact hypertrophied heart, all possible arrhythmogenic factors, resulting in non-uniform conduction, non-uniform repolariza- 
tion and focal mechanisms are present. To evaluate the in vivo importance of cellular adaptations, like the prolongation of the repolarization and the suggested increased propensity for triggered arrhythmias, a special, clinically applicable technique and a reproducible animal model of homogeneous hypertrophy is needed.

\section{Monophasic action potentials}

One technique that could possibly be of help to more accurately assess the time course of repolarization in the intact heart is the registration of monophasic action potentials. ${ }^{102-}$ ${ }^{105}$ These are extracellularly recorded wave forms with a striking similarity to transmembrane action potentials recorded with micro-electrodes. ${ }^{102}$ Initially this technique was developed to more accurately assess the time course of repolarization (action potential duration) than possible with conventional intracardiac recordings or the ECG. In 1966, the first monophasic action potentials were obtained in patients by means of a suction electrode. However, in the eighties, a simpler and safer technique for monophasic action potential recordings using contact pressure was introduced. ${ }^{103-105}$ With the development of the monophasic action potential registration the possibility to visualize afterdepolarizations in the intact heart was created.

To evaluate the propensity of the hypertrophied canine heart to triggered arrhythmias, the monophasic action potential recording method was applied in the dog with biventricular hypertrophy due to complete atrioventricular-block. For over 15 years, we have been using this model to study ventricular arrhythmias with special emphasis on triggered arrhythmias.

\section{Goals of the thesis}

Our first goal was to be able to identify delayed afterdepolarization-dependent triggered arrhythmias in the intact heart. Therefore, the endocardial recording of monophasic action potentials was combined with pacing (chapter 2). This provided a method to visualize delayed afterdepolarization-dependent triggered arrhythmias. Secondly, the occurrence and maintenance of delayed afterdepolarization-dependent triggered arrhythmias was related to the behavior of the diastolic slope of the monophasic action potential signal (chapter 3). Thirdly, the effect of suppressing elevated levels of intracellular calcium with ryanodine was shown to be effective in suppression of delayed afterdepolarization-dependent triggered arrhythmias (chapter 4). 
Table 4: Factors promoting arrhythmias in (de)compensated hypertrophy

Non-uniform Non-uniform Focal

conduction repolarization

Structural

Scar

Fibrosis

Ischemia

Dilatation

Hypertrophy

Calcium dyshandling

$+$ $+$ $+\quad+$ $+\quad+\quad+\frac{4}{3}$ $+\quad+$ $+$

Neurohumoral

High sympathetic tone

$+$ $?$ $+$

Electrolyte disturbances

Hypokalemia

Hypomagnesemia

Pharmacological treatment

Diuretics

Digitalis

Inotropic drugs

Anti-arrhythmic drugs 
After we were able to visualize delayed afterdepolarizations in the intact heart, we aimed to obtain more information regarding the structural, neurohumoral, electrophysiological and functional adaptations that occur after the creation of atrioventricular-block (chapter 5) and related this to the induction of Torsade de Pointes arrhythmias. More recently, experiments were performed to demonstrate that functional adaptations are associated with the accurrence of delayed afterdepolarization-dependent triggered arrhythmias (chapter 6). These experiments raised questions regarding the ability of the hypertrophied heart to maintain its pump function during chronotropic stress, which was addressed in chapter 7 .

\section{References}

1. Widdershoven J, Gorgels A, Vermeer F, Dijkman L, Verstraaten G, Dassen W, Wellens H. Changing characteristics and in-hospital outcome of patients admitted with acute myocardial infarction. Observations from 1982-1994. Eur Heart $J$ 1997; 18: 1073-1080.

2. Kannel WB, Belanger AJ. Epidemiology of heart failure. Am Heart J 1991; 121: 951-957.

3. Reitsma JB, Mosterd A, Koster RW, van Capelle FJL, Grobbee DE, Tijssen JGP. Stijging van het aantal opnamen wegens hartfalen in Nederlandse ziekenhuizen in de periode 1980-1992. Ned Tijdschr Geneeskd 1994; 138: 866-871.

4. Gradman A, Deedwania P, Cody R, Massie B, Packer M, Pitt B, Goldstein S. Predictors of total mortality and sudden death in mild to moderate heart failure. JAm Coll Cardiol 1989; 14:564-570.

5. McKee PA, Castelli WP, McNamara PM, Kannel WB. The natural history of congestive heart failure: The Framingham study. New Engl J Med 1971; 285: 1441-1446.

6. Stevenson WG, Stevenson LW, Weiss J, Tillisch JH. Inducible ventricular arrhythmias and sudden death during vasodilator therapy of severe heart failure. Am Heart $J$ 1988; 116: 1447-1454.

7. Chakko CS, Gheorghiade M. Ventricular arrhythmias in severe heart failure: Incidence, significance, and effectiveness of anti-arrhythmic therapy. Am Heart J1985; 109: 497-504.

8. Wilson JR, Schwartz S, St John Sutton M, Ferraro N, Horowitz LN, Reichek N, Josephson ME. Prognosis in severe heart failure: relation to hemodynamic measurements and ventricular ectopic activity. I Am Coll Cardiol 1983; 2: 403-410.

9. The Consensus Trial Study Group. Effects of enalapril on mortality in severe congestive heart failure. New Eng J Med 1987; 316: 1429-1435.

10. Hallstrom A, Pratt CM, Greene HL, Huther M, Gottlieb S, DeMaria A, Yuong JB. Relations between heart failure, ejection fraction, arrhythmia suppression and mortality: Analysis of the cardiac arrhythmia suppression trial. $J$ Am Coll Cardiol 1995; 25: 1250-1257.

11. Levy D. Echocardiographically detected left ventricular hypertrophy: Prevalence and risk factors. Ann int Med 1988; 108: 7-13.

12. Kannel WB. Left ventricular hypertrophy as a risk factor in arterial hypertension. Eur Heart $J 1992$; 13: D82-D88.

13. Klein RC. Ventricular arthythmias in aortic valve disease: Analysis of 102 patients. Am J Cardiol 1984; 53: 1079-1083. 
14. McLemachan JM, Henderson E, Morris KI, Dargie HJ. Ventricular arrhythmias in patients with hy: pertensive left ventricular hypertrophy. New Engl J Med 1987: 31 7: 787-792.

15. Messerli FH, Ventura HO, Elizardi DJ, Dunn FG, Frohlich ED Hypertension and sudden death. Increased ectopic activity in left ventricular bypertrophy. Am Med $J 1984 ; 77: 18-22$.

16. Levy D, Anderson KM, Savage DD, Balku SA, Kannel BW, Castelli WP. Risk of ventricular arrhythmias in left ventricular hypertrophy: The Framingham heart study. Am JCardiol 1987; 60: 560.565 .

17. Kannel WB, Grodon T, Offutt D. Left ventricular hypertrophy by electrocardiogram. Prevalence, incidence and mortality in the Framingham Study. Ann Int Med 1969; 71:89-105.

18. Ghali JK, Kadakia SK, Cooper RS, Liao Y. Impact of left ventricular arrhythmias in the absence of coronary artery disease. $J$ Am Coll Cordiol 1991; 17: 1277-1282.

19. Francis GS. Development of arrhythmias in the patient with congestive heart failure: Pathophysiology, prevalence and prognosis. Am J Cardiol 1986; $57: 3-7$.

20. Packer M. Lack of relation between ventricular arrhythmias and sudden death in patients with chronic heart failure. Circulation 1992; 85: 150-156.

21. Doval HC, Nul DR, Grancelli HO, Varini SD, Soifer S, Corrado G, Dubner S, Scapin O, Perrone SV. Nonsustained ventricular tachycardia in severe heart failure. Independent marker of increased mortality due to sudden death. Circulation 1996; 94:3198-3203.

22. Liu CP, Ting CT, Lawrence W, Maughan L, Chang M-S, Kass DA. Dim inished contractile response to increased heart rate in intact human left ventricular hypertrophy. Circulation 1993; 88: 1893 1906.

23. Seed WA, Noble MIM, Walker JM, Miller GAH, Pidgeon J, Redwood D, Wanless R, Franz MR, Schoettler M, Schaeffer J. Relationships between beat-to-beat interval and the strength of contraction in the healthy and the diseased human heart. Circulation 1984; 70:799-805.

24. Gaasch WH, Zile MR., Hoshino PK, Apstein CS, Blaustein AS. Stress-shortening relations and myocardial blood flow in compensated and failing canine hearts with pressure overload hypertrophy. Cinculation 1989; 79:872-883.

25. Carabello BA, Mee RM. Collins JJ, Kloner RA, Levin D, Grossman W. Contractile function in chronic gradually developing subcoronary stenosis. Am J Physiol 1981; 240: H80-H86.

26. Hittinger L, Patrick T, Ihara T, Hasebe N, Shen YT, Kalthof B, Shannon RP. Exercise induces cardiac dysfunction in both moderate, compensated and severe hypertrophy. Circulation 1994;89: 2219-2231.

27. Hasebe N, Hittinger $\mathrm{L}$, Kohin $\mathrm{S}_{\text {, Shen }} \mathrm{YT}$, Graham RM, Vatner SF. Effects of hypertrophy on left atrial and ventricular compliance and plasma ANF levels in conscious dogs. Am JPhysiol 1995; 268: H781-H787.

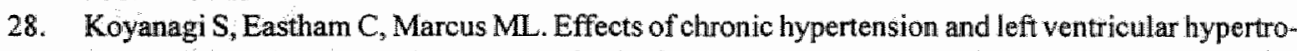
phy on the incidence of sudden cardiac death after coronary artery occlusion in conscious dogs. Cir. culation 1982; 65: 1:92-1197.

29. Laks MM, Morady F, Garner D, Swan HIC. Relation of ventricular volume, compliance, and mass in normal and pulmonary arterial banded canine heart. Cardiovasc Res 1972; 6: 187-198. 
30. Ben-David J, Zipes DP, Ayers GM, Pride HP. Canine left ventricular hypertrophy predisposes to ventricular tachycardia induction by phase 2 early afterdepolarizations after administration of BAY K 8644. I Am Coll Cardiol 1992; 20: 1576-1584.

31. Carabello BA, Zile MR, Tanaka $R$, Cooper IV $\mathrm{G}$. Left ventricular hypertrophy due to volume overload versus pressure overload. Am J Physiol 1992; 263: H1 137-H1144.

32. Urabe $Y$, Mann DL, Kent RL, Nakano K, Tomanek RJ, Carabello B, Cooper $\mathbb{I V ~ G . ~ C e l l u l a r ~ a n d ~ v e n - ~}$ tricular contractile dysfunction in experimental canine mitral regurgitation. Circ Res 1992; 70; 131 147.

33. Himura Y, Felten SY, Kashiki M, Lewandowski TJ, Delehanthy JM, Liang CS. Cardiac noradrenergic nerve terminal abnormalities in dogs with experimental congestive heart failure. Circulation 1993; 88: 1299-1309.

34. LeWinter MMy Engler RI, Karliner IS, Enhanced left ventricular shortening during chronic volume overload in conscious dogs. Am $J$ Physiol 1980; 238: H126-H133.

35. Weber KT, Pick R, Silver MA, Moe GW, Janicki JS, Zucker IH, Amstrong PW. Fibrillar collagen and remodeling of the dilated canine left ventricle. Circulation 1990;82:1387-1401.

36. Turina M, Bussman WD, Krayenbuh.l HP. Contractility of the hypertrophied canine heart in chronic volume overload. Cardiovasc Res 1969; 3:486-495.

37. Starzl TE, Gaertner RA, Baker RR. Acute complete heart block in dogs. Circulation 1955; 12: 8289.

38. Newman WH. Contractile state of hypertrophied left ventricle in long-standing volume overload. Am J Pipsiol 1978; 234: H88-H93.

39. Brockman SK. Cardiodynamics of complete heart block. Am J Cardiol 1965; 16: 72-83.

40. Wusten $B$, Flameng $W$, Schaper $W$. Cardiac function in the chronically volume-overloaded canine heart. Basic Res Cardiol 1977; 72: 172-177.

41. Wusten B, Flameng W, Winkler B, Schaper W. Role of cardiac contractility in hypertrophy from chronic volume loading. Candiovasc Res 1977; 11: 132-140.

42. Moe GW, Stopps TP, Angus C, Forster C, De Bold AJ, Amstrong PW. Alterations in serum sodium in relation to atrial natriuretic factor and other neuroendocrine variables in experimental pacing-induced heart failure. $J$ Am Coll Cardiol 1989; 13: 173-179.

43. Perreault CL, Shannon RP, Komamura K, Vatner SF, Morgan JP. Abnormalities in intracellular calcium regulation and contractile function in myocardium from dogs with pacing-induced heart failure. J Clin Invest 1992; 89: 932-938.

44. Amstrong PW, Stopps TP, Ford SE, De Bold AJ. Rapid ventricular pacing in the dog: pathophysiologic studies of heart failure. Circulation 1986; 74: 1075-1084.

45. Spinalle FG, Holzgreve HH, Mukherjee R, Arthur SR, Child MJ, Powell JR, Koster WH. Left ventricular and myocyte function after early recovery from tachycardia induced cardiomyopathy. Am J Physiol 1995; 268: H836-H847.

46. Sabbal HN, Stein PD, Kono T, Gheorghiade M, Levine TB, Jafri S, Hawkins ET, Goldstein S. A canine model of chronic heart failure produced by multiple sequential coronary microembolizations. An J Physiol 1991; 260: H1379-H1384.

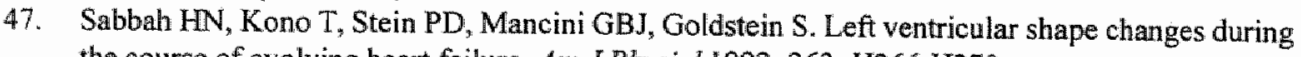
the course of evolving heart failure. Am J Physiol 1992; 263: H266- H270. 
48. McDonald KM, Mock J, D Aloia A, Parrish T, Hauer K, Francis GS, Stillman A, Cohn JN. Bradykinin antagonism inhibits the antigrow th effect of converting enzyme inhibition in the dog myocardium after discrete transmural myocardial necrosis. Circulation 1995; 91 : 2043-2048.

49. McDonald KM, Francis GS, Carlyle PF, Hauer K, Matthews J, Hunter DW, Cohn JN. Hemodynam ic, left ventricular structural and hormonal changes after discrete myocardial damage in the dog. J Am Coll Cardiol 1992; 19:460-467.

50. Laks MM, Morady $F$, Swan HJC. Myocardial hypertrophy induced by chronic infusion of subhypertensive doses of norepinephrine in the dog. Chest $1973 ; 64: 75-78$.

51. Legault $F$, Rouleau $\mathbb{L}$, Juneau $C$, Rose $C$, Rakusan $K$. Functional and morphological characteristics of compensated and decompensated cardiac hypertrophy in dogs with chronic infrarenal aortocaval fistulas. Circ Res 1990; 66: 846-859.

52. Pak PH, Nuss B, Tunin RS, Kaab S, Tomaselli GF, Marban E, Kass DA. Repolarization abnormalities, arrhythmia and sudden death in canine tachycardia-induced cardiomyopathy. $J \mathrm{~A}$ Coll Cardiol 1997; $30: 576-584$.

53. Sabbah HN, Goldberg DG, Schoels W, Kono T, Webb C, Brachmann J, Goldstein S. Spontaneous and inducible ventricular arrhythmias in a canine model of chronic heart failure: Relation to haemodynamics and sympathoadrenergic activation. Eur Heart $J 1992 ; 13 ; 1562-1572$.

54. Li HG, Jones DL, Yee R, Klein GJ Electrophysiologic substrate associated with pacing-induced heart failure in dogs: potential value of programmed stimulation in predicting sudden death. $J \mathrm{Am}$ Coll Cardiol 1992; 444-449.

55. LiHG, Jones DL, Yee R, Klein GJ. Arrhythmogenic effects of catecholamines are decreased in heart failure induced by rapid pacing in dogs. Am J Physiol 1993; 265: H1654-H1662.

56. Martins JB, Kim W, Marcus M. Chronic hypertension and left ventricular hypertrophy facilitate the induction of sustained ventricular tachycardia in dogs 3 hours after left circumflex coronary artery occlusion. I Am Coll Cardiol 1989; 14: 1365-1373.

57. Dellsperger $\mathrm{KC}$, Martins $\mathrm{JB}$, Clothier $\mathbf{\Omega}$, Marcus $\mathrm{ML}$. Incidence of sudden cardiac death associated with coronary artery occlusion in dogs with hypertension and left ventricular hypertrophy is reduced by chronic beta-adrenergic blockade. Circulation 1990; 82: 941-950.

58. Kääb S, Nuss HB, Chaimvimonvat N, ORourke B, Pak PH, Kass DA, Marban E, TomaselliGF. Ionic mechanism of action potential prolongation in ventricular myocytes from dogs with pacing. induced heart failure. Circ Res 1996; 78: 262-273.

59. Cameron JS, Myerburg RJ, Wong SS, Gaide MS, Epstein K, Alvarez TR, Gelband H, Guse PA, Bassett AL. Electrophysiologic consequences of chronically experimentally induced left ventricuLar pressure overload. J Am Coll Cardial 1983; $2: 481-487$.

60. Aronson RS, Ming Z. Cellular mechanisms of arrhythmias in the hypertrophied and failing myocardium. Circulation 1993; 87: VII76-VII83.

61. Tomaselli GF, Beuckelmann DJ, Calkins HG, Berger RD, Kessler PD, Lawrence JH, Kass D, Feldman AM, Marban E. Sudden cardiac death in heart failure, the role of abnormal repolarization. Circulation 1994; 90: 2534-2539.

62. Hoffman BF, Rosen MR. Cellular mechanisms for cardiac arrhythmias. Circ Res 1981; 49:1-15.

63. Dillon SM, Allessie MA, Ursell PC, Wit AL. Influences of anisotropic tissue structure on reentrant circuits in the epicardial border of subacute infarcts. Circ Res 1988; 63: 182-206. 
64. Allessie MA, Bonke FM, Schopman FJG. Circus movement in rabbit atrial muscle as a mechanism of tachycardia. III. The "leading circle" concept; a new model of circus movement in cardiac tissue without the involvement of anatomic obstacle. Circ Res 1977; 41:9-18.

65. Durrer D, Schoo L, Schuilenburg RM, Wellens HJ. The rolle of premature beats in the initiation and termination of supraventricular tachycardia in the Wolff-Parkinson-White syndrome. Circulation $1967 ; 34: 644-662$.

66. Wellens HJJ. Value and limitations of programmed electrical stimulation of the heart in the study and treatment of tachycardias. Circulation $1978 ; 57: 845-851$.

67. Rosen MR. Is the response to programmed electrical stimulation diagnostic of mechanisms for arrhythmias? Circulation 1986; 73: II1 18-I127.

68. Dangman KH, Hoffman BF. Studies on overdrive stimulation of canine cardiac purkinje fibers: maximal diastolic potential as a determinant of the response. $J \mathrm{Am}$ Coll Cardiol 1983;2:1183-1190.

69. Vos MA, Gorgels AP, Leunissen JD, Havenith MG, Kriek E, Smeets JL, Wellens HJ. Programmed electrical stimulation and drugs to identify two subgroups of ventricular arrhythmias occurring 1624 hours after occlusion of the left anterior descending artery. Circulation 1992; 85: 747-755.

70. Cranefield PF, Aronson R: Cardiac arrhythmias: The role of triggered activity and other mechanisms. New York., Nt Kisco, New York, Futura publishing Co., 1988

71. Vos MA, Verduyn SC, Gorgels AP, Lipcsei GC, Wellens HJ. Reproducible induction of early afterdepolarizations and torsade de pointes arrhythmias by $\mathrm{d}$-sotalol and pacing in dogs with chronic atrioventricular block. Circulation 1995; $91: 864-72$.

72. Verduyn SC, Vos MA, van der Zande J, van der Hulst FF, Wellens HJJ. Role of interventricular dispersion of repolarization in acquired Torsade de Pointes arrhythmias: Reversal by magnesium. Cardiovasc Res 1997; 34: 453-463.

73. Damiano BP, Rosen MR. Effects of pacing on triggered activity induced by early afterdepolarizations. Circulation 1984; 69: 1013-1025.

74. Daniels MC, ter Keurs HE. Suppressive effect of R 56865 on triggered propagated contractions and triggered arrhythmias in rat cardiac trabeculae. I Cardiovasc Pharmacol 1992; 20: 187-196.

75. Ehara T, Noma A, Ono K. Calcium-activated non-selective cation channel in ventricular cells isolated from adult guinea-pig hearts. $J$ Physiol (Lond) 1988; 403:117-133.

76. BenndorfK, Biskup C, Friedrich M. Voltage-dependent kinetics of Na-Ca exchange current in Ca2t=loaded cells. Am J Physiol 1993; 265: C1258-C1265.

77. Lipp P, Pott L. Transient inward current in guinea-pig atrial myocytes reflects a change of sodiumcalcium exchange current. $J$ Physiol (Lond) $1988 ; 403: 355-366$.

78. Wit AL, Rosen MR: Afterdepolarizations and triggered activity: distinction from automaticity as an arr hythmogenic mechanism. The heart and cardiovascular system. Edited by Fozzard HA, Haber E, Jennings RB, Katz AM, Morgan HE. New York, Raven Press, 1992, pp $2113-2163$

79. Voldiers PGA, Kulcsár A, Vos MA, Sipido KR, Wellens HJJ, Lazzara R, Szabo B. Similarities between early and delayed afterdepolarizations induced by isoproterenol in canine ventricular myocytes. Cardiovasc Res 1997 ; 34: 348-359.

80. Priori SG, Corr PB. Mechanisms underlying early and delayed afterdepolarizations induced by catecholamines. Am J Physiol 1990; 258: H1796-H1805.

81. Yamada $\mathrm{KA}_{i}$ Corr $\mathrm{PB}$. Effects of beta-adrenergic receptor activation on intracellular calcium and membrane potential in adult cardiac myocytes. J Cardiowasc Electrophysiol 1992; 3: 209-224. 
82. Gorgels AP, Vos MA, Smeets JL, Wellens HJ. Ventricular arthythmias in heart failure. A $m J$ Cardiol 1992; 70: 37C-43C.

83. De Biakker JMT, Van Capelle FJL, Janse MJ, Wilde AAM, Coronel R, Becker AE, Dingemans KP, Van Hemel NM, Hauer RNW. Reentry as cause of ventricular tachycardia in patients with chronic ischemic heart disease: electrophysiologic and anatomical correlations. Circulation 1988; 77 : 589 606.

84. Ferrier GR, Moffat MP, Lukas A. Possible mechanisms of ventricular arrhythmias elicited by ischemia followed by reperfusion. Studies on isolated canine ventricular tissues. Circ Res 1985; 56: 184-94.

85. Reiter MJ. Pathophysiology of ventricular arrhythmias in patients with congestive heart failure. Heart Failure 1989; 5: 155-166.

86. Dear JW, Lab MJ. Arrhythmia a in heart failure: Role of mechanically induced changes in electrophysiology. Lancet 1989; 8650: 1309-1312.

87. Janse MJ, Wit AL. Electrophysiological mechanisms of ventricular arrhythmias resulting from myocardial ischemia and infarction. Physiol Rev 1989; 69: 1049-1169.

88. Levine $\mathrm{JH}$, Guarnierni T, Kadish $\mathrm{AH}$. Changes in myocardial repolarization in patients undergoing balloon valvuloplasty for congenital pulmonary stenosis: Evidence for contraction-excitation feedback in thumans. Circulation 1988; 77: 70-77.

89. Ferrier GR. The effects of tension on acetylstrophantidine-induced transient depolarizations and aftercontractions in canine myocardial and purkinje tissue. Circ Res 1976; 38:156-162.

90. El-Sherif N, Gough WB, Zeiler RH, Mehra R. Triggered ventricular rhythms in 1-day-old myocardial infarction in the dog. Circ Res $1983 ; 52: 566-579$.

91. Le Marec $\mathrm{H}$, Dangman $\mathrm{KH}_{3}$, Danilo $\mathrm{P}$, Rosen MR. An evaluation of automaticity and triggered activity in the canine heart one to four days after myocardial infarction. Circulation 1985; 71: 122436.

92. Brugada P, Wellens HJJ. Early afterdepolarizations: Role in conduction block, prolonged repolarization-dependent re-excitation, and tachyarrhythmias in the human heart. PACE 1985;8:889-896.

93. Gwathmey JK, Copelas L, MacKinnon R, Schoen FJ, Feldman MD, Grossman W, Morgan JP. Abnormal intracellular calcium handling in myocardium from patients with end-stage heart failure. Circ Res 1987; 61: 70-76.

94. Arai $\mathrm{M}$, Matsui $\mathrm{H}$, Periasamy M. Sarcoplasmic gene expression in cardiac hypertrophy and heart failure. Circ Res 1994; 74: 555-564.

95. Nordin $\mathrm{C}$. Abnormal calcium handling and the generation of ventricular arrhythmias in congestive heart failure. Heart Failure 1989; 5: 143-154.

96. Beuckelmann DJ, Näbauer $M$ * Erdmann E. Intracellular calcium handling in isolated ventricular myocytes from patients with terminal heart failure. Circulation 1992; 85: 1046-1055.

97. Gengo PJ, Sabbah HNN, Steffen RP, Sharpe JK, Kono T, Stein PD, Goldstein S. Myocardial beta-adrenoreceptor and voltage sensitive calcium channel changes in a canine model of chronic heart failure. J Mol Cell Cardiol 1992; 24: 1361-1369.

98. Levy MN, Wiseman MN. Electrophysiologic mechanisms for ventricular arrhythmias in left ventricular dysfunction: Electrolytes, catecholamines and drugs. J Clin Pharmacol 1991; 31 : 1053 1060.

99. Smith TW. Digitalis. Mechanisms of action and clinical use. N Engl J Med 1988; 318: 358-65. 
100. Steness $\mathrm{E}_{\text {, Olesen }}$ K. C. Cardiac amythmias induced by hypokalemia and potassium loss during: mantenance digoxin therapy. Br Hear $J 1976 ; 38: 167-172$.

101. Ranger $S$, Talajuc $M_{\text {, Lemery }}$, Roy $D$, Nattel $S$. Amplification of fecainide induced ventricutar conduction slowing by exercise. Circulation 1989; 79: 1000-1006.

102. Hofman BF, Cranefreld PF, Lepeschikn $\mathrm{E}_{\text {s }}$ Surawicz B, Herthch HC. Comparison of cardiac monophasic action potentials recorded by intracelluhar and suction electrodes. Am I Physiol 1959; 196: 1297-1301.

303. Franz MR, Burhoff D, Spurgeon 1 . Weisfeldt ML, Lakatta EG. In vitro validation of a new cardiac catheter technique for recording monophasic action potentials. Eur Heart $J 1986 ; 7 ; 34-41$.

104. Franz MR. Bridging the gap between basic and elinical electrophysiology: What can be learned from monophasic action potential recordings J Cardiovasc Electrophysiol 1994; 5: 699-710.

105. Olsson SB, Blomstrom P, Blomstron LC, Wohlfart B. Endocardial monophasic action potentials. Correlations with intracellalar electrical activity. An N Y Acad Sc; 1990; 601: 119-27.

106. Omens $\mathrm{H}$, Cowell IW. Transmural distribution of myocardial tissue growth induced by volumeoverload Typertrophy in the dog. Circulation 1991; 84: 1235-1245.

107. Villarrea D, Freeman $\mathrm{RH}$, Johnson RA. Neurohumoral modulators and sodium balance in experimental heart failure. An IPlysiol 1993; 264: H1 187-H1193. 


\section{Chapter 2}

\section{Combining monophasic action potential recordings with pacing to demonstrate delayed afterdepolarizations and triggered arrhythmias in the intact heart \\ Value of the diastolic slope}

Marieke de Groot

Marc Vos

Anton Gorgels

Jet Leunissen

Bert van der Steld

Hein Wellens

Abstract presented at the American College of Cardiology 1994 (J Am Coll Cardiol 1994; 466A)

Published in Circulation 1995; 92: 2697-2704 


\section{Abstract}

Introduction: In the intact heart, methodological difficulties hamper the direct visualization of delayed afterdepolarizations responsible for triggered arrhythmias. Therefore, we tested the hypothesis that a combination of pacing and the recording of a monophasic action potential could facilitate the recognition of oubain induced delayed afterdepolarizations and triggered arrhythmias by demonstrating an increase in the diastolic baseline slope (dV/dt) of the monophasic action potential recording at the end of a pacing train.

Methods: In anesthetized dogs with chronic atrioventricular-block, a right ventricular endocardial monophasic action potential was recorded during 1) control $(n=11), 2) 15-45$ minutes after ouabain $(45 \pm 10 \mu \mathrm{g} / \mathrm{kg}, \mathrm{n}=11), 3) 10$ minutes after lidocaine $(3 \mathrm{mg} / \mathrm{kg}, \mathrm{n}=5)$ and 4) during lidocaine wash-out $(n=3)$. Pacing was performed with the monophasic action potential catheter. Additionally, the protocol was performed in 3 dogs with conducted sinus rhythm during control and ouabain circumstances.

Results: During control, the slope value was $2 \pm 2 \mathrm{mV} / \mathrm{s}$ (mean $\pm \mathrm{SD}$ ), the incidence of delayed afterdepolarizations after the stimulation train was $6 \%$ and no ventricular tachycardias were induced in dogs with atrioventricular-block. During ouabain the slope and delayed afterdepolarization incidence increased to respectively $26 \pm 14 \mathrm{mV} / \mathrm{s}$ and $74 \%$ ( $p<0.05$, for both). Ventricular tachycardias were induced frequently. Lidocaine prevented ventricular tachycardia induction by decreasing the slope and the incidence of delayed afterdepolarizations. This effect disappeared after lidocaine wash-out. During conducted sinus rhythm, similar results were found.

Conclusion: By combining pacing and monophasic action potential recordings, the diastolic slope observed on monophasic action potential recordings in ouabain-intoxicated hearts can be used as a marker for delayed afterdepolarizations and triggered arrhythmias. This finding may be helpful in identifying triggered activity in the intact heart.

\section{Introduction}

The arrhythmogenic mechanism triggered activity consists of two subgroups: early or delayed afterdepolarizations. ${ }^{1-5}$ Although many attempts have been made to identify delayed afterdepolarizations as the cause of clinical tachycardias, ${ }^{6-16}$ their relevance is still poorly understood. A direct approach could be the visualization of delayed afterdepolarizations through the recording of endocardially placed monophasic action poten- 

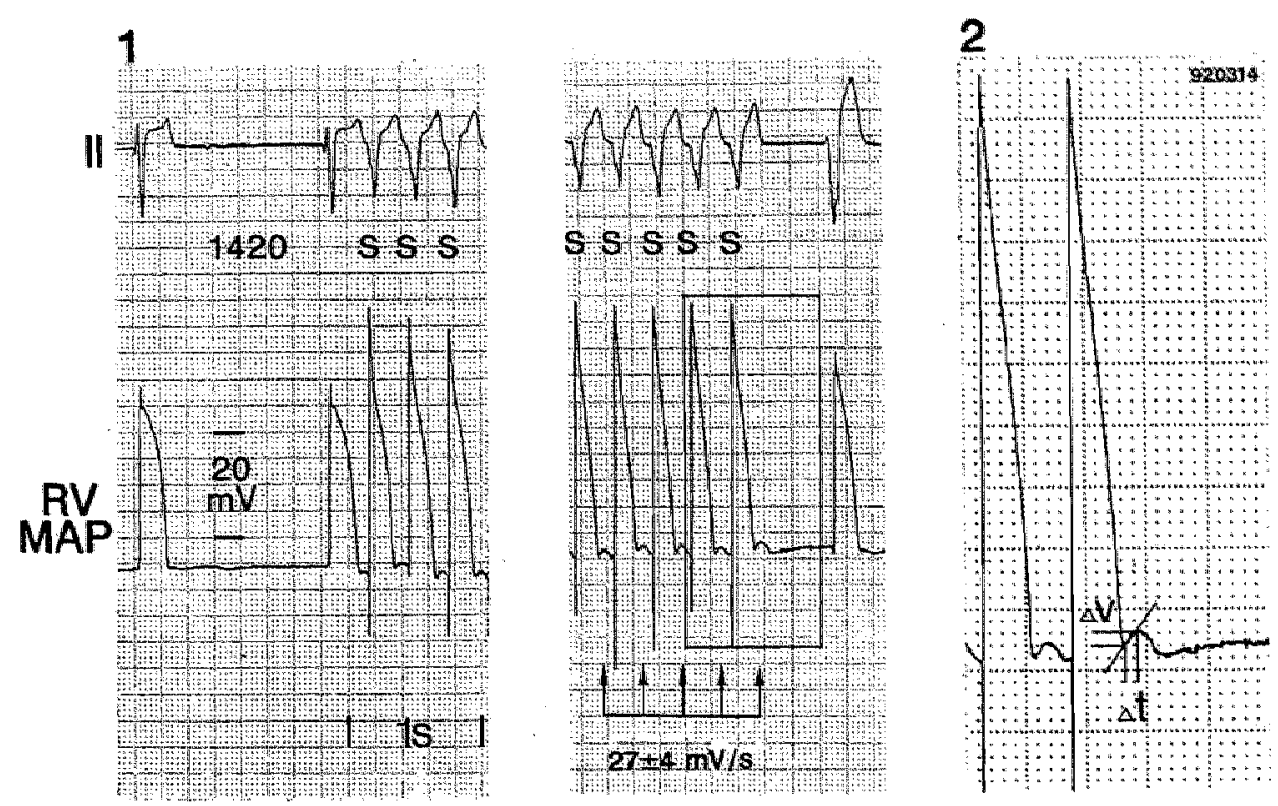

\section{$n(V s)=33 \quad V s-V s=300 m s$}

Figure 1: Determination of the diastolic slope in a monophasic action potential recording.

Illustration of the method used to determine the slope in the diastolic interval of a monophasic action potential (MAP) signal during pacing. One ECG-lead (II) and the MAP-signal, recorded endocardially in the right ventricle, are shown. Panel 1: The spontaneous idioventricular rhythm is interrupted by ventricular pacing, indicated by $S$, with an interstimulus interval (Vs-Vs) of $300 \mathrm{~ms}$ and 33 stimuli (nVs). This is a continuous registration in which the 25 beats in the middle of the pacing train are left out. During the first 30 min after ouabain administration, delayed afterdepolarizations (DADs) are clearly visible in between the paced beats and at the end of the paced train. Slope is calculated for the 5 last paced complexes. In panel 2, the indicated fragment of panel 1 is enlarged. The line drawn in this panel, indicates the slope. The difference in vertical and horizontal axis, which is used for the calculation of the slope, is represented as $\mathrm{dV}$ (change in voltage) and $\mathrm{dt}$ (change in time).

tials. This approach has been successfully applied in animals during (non-sustained) ventricular tachycardias ${ }^{17-20}$ and more recently during atrial tachycardias in patients. ${ }^{16}$ However, methodological difficulties still hamper the general use of this technique. ${ }^{21,22}$ To elucidate delayed afterdepolarizations as the substrate of tachycardia initiation, we hypothesized, based on the frequency dependence of delayed afterdepolarizations, that their occurrence is indicated by the presence of an increase in the baseline slope $(\mathrm{dV} / \mathrm{dt})$ in the monophasic action potential recordings of the last beats of pacing. This increase 
in slope has been observed in action potentials recorded in isolated tissue ${ }^{5}$ prior to induction of (triggering) delayed afterdepolarizations. Although the slope of the ascending limb of delayed afterdepolarizations has been calculated, ${ }^{23,24}$ the slope during pacing has never been quantified. If our hypothesis is correct, it might provide a tool to identify triggered activity in the intact heart, without the necessity to induce ventricular tachycardia.

\section{Methods}

\section{General}

All experiments were performed in accordance with the "Guiding principles in the care and use of animals" as approved by the American Physiological Society and under the regulations of the Committee for Experiments on Animals of the University of Limburg, Maastricht, The Netherlands.

The studies were performed in 11 anesthetized dogs (mean body weight of $25 \pm 5 \mathrm{~kg}$ ) of either sex. Complete atrioventricular-block was made to exclude the interference of conducted sinus beats on ventricular arrhythmias. ${ }^{25}$ During the atrioventricular-block operation an epicardial screw-in electrode (Bakken Research Center, Maastricht, The Netherlands) was placed on the apex of the left ventricle. The experiments were performed at least 2 weeks after this operation. Additionally, 3 anesthetized dogs with normally conducted sinus rhythm, were also studied.

Stimulation was done with a programmable stimulator having a synchronizing circuit. Six surface ECG leads and one monophasic action potential recording were simultaneously registered on a monitor and stored on an optical disc. Drugs were administered through a cannula in a cephalic vein.

\section{Anesthesia}

Experiments were performed under anesthesia and sterile conditions. Premedication $(0.2$ $\mathrm{ml} / \mathrm{kg}$ ) was given intramuscularly with a drug mixture containing $10 \mathrm{mg}$ oxycodan $\mathrm{HCl}$, $1 \mathrm{mg}$ acepromazine and $0.5 \mathrm{mg}$ atropine sulfate per $\mathrm{ml}$. An antibiotic ( $1000 \mathrm{mg}$ ampicillin, Amfipen $^{\mathrm{R}}$, Gist Brocades) was given preoperatively (IV) and postoperatively (SC). Anesthesia was induced by sodium pentobarbital ( $20 \mathrm{mg} / \mathrm{kg}$ intravenous). The dogs were artificially ventilated (Pulmomat respirator, Dräger), through a cuffed endotracheal tube with a mixture of oxygen, nitrous oxide and halothane (vapor concentration $0.5-1.0 \%$ ). Ventilation was adjusted on the basis of continuous monitoring of the carbon dioxide 
concentration of the expired air. A thermal mattress was used to maintain body temperature around $37^{\circ} \mathrm{C}$ during the experiments.

\section{Recording of monophasic action potentials}

In order to register an endocardial monophasic action potential signal, a quadripolar contact electrode ${ }^{26,27}$ (Steerable Franz ${ }^{\mathrm{TM}}$ Combination Catheter, EP technologies), was placed endocardially in the right ventricle. The 7 French monophasic action potential catheter was introduced under fluoroscopy, through the femoral or external jugular vein. The signals were amplified using a customized isolated DC-coupled differential amplifier with a $20 \mathrm{mV}$ calibration pulse. The offset of this amplifier is variable and can be adjusted to the recorded signal. Sampling was done at a rate of $1 \mathrm{kHz}$ per signal.

Monophasic action potential phases were divided according to the definitions used for the transmembrane action potential. Amplitude was defined as the difference between phase 4 and 2 of the signal. The minimally accepted amplitude of the monophasic action potential was $15 \mathrm{mV} .{ }^{17,18}$ If the monophasic action potential had a lower amplitude under baseline conditions the catheter was moved to an adjacent side. To make sure that the recording place of the catheter could be repeatedly reached throughout the experiment, the catheter was (re)placed at the same location at least 3 times before the experiment was started. This was achieved using guidance from the fluoroscopy monitor and through pacing using the monophasic action potential catheter.

To avoid artefacts during phase 4 of the action potential, ${ }^{27}$ rigorous attention was paid to record stable diastolic potentials. Furthermore, the monophasic action potential had to have a constant configuration and a smooth shape during control circumstances.

\section{Experimental protocol}

A protocol was used which we routinely employ in conscious dogs. ${ }^{19,28}$ Pacing was performed from the monophasic action potential catheter with a strength of twice diastolic threshold and a pulse width of $2 \mathrm{~ms}$ and consisted of 3 stimulation trains with an interstimulus interval of $300 \mathrm{~ms}$ and a duration of 4,10 , or 20 seconds. This resulted in 3 pacing trains in every dog, both during control ( 11 atrioventricular-block dogs and 3 sinus rhythm dogs) and during each intervention. Between these pacing trains, enough time was allowed for the heart to regain its pre-pacing rhythm. After finishing control pacing, a bolus of ouabain, in relation to body weight, ${ }^{28}$ (mean $45 \pm 10 \mu \mathrm{g} / \mathrm{kg}$ ) was intravenously administered over 1 minute. Ten minutes later this was followed by 
continuous infusion ${ }^{29}$ (mean $0.08 \pm 0.02 \mu \mathrm{g} / \mathrm{kg} / \mathrm{min}$ ) during the whole experiment. Pacing was resumed $15 \mathrm{~min}$ after the bolus of ouabain. ${ }^{28}$

Five out of the 11 atrioventricular-block dogs received an intravenous bolus of lidocaine ( $3 \mathrm{mg} / \mathrm{kg} / 2$ minutes) to reverse the ouabain effects and to exclude possible artefacts in the monophasic action potential recordings. The pacing protocol was repeated within 10 minutes after lidocaine. Finally, in 3 dogs the pacing protocol was repeated once more after a wash-out period of lidocaine.

In 5 atrioventricular-block dogs, the above described pacing protocol was expanded with 3 pacing trains having an interstimulus interval of $400 \mathrm{~ms}$, using the same durations ( 4 , 10 and 20 seconds).

\section{Data analysis}

For off-line analysis of the recorded signals, a customized ECGVIEW program was used, which contains a special module for the analysis of monophasic action potential signals on a beat to beat basis. It can calculate the amplitude, the duration and the slope ( $\mathrm{dV} / \mathrm{dt}$ ) of a selected monophasic action potential signal. Slope was calculated as the mean rate of rise $\left(\mathrm{dV} / \mathrm{dt}\right.$ ) of the ascending limb of the delayed afterdepolarization ${ }^{22,23}$ or as the mean change in diastolic potential in between the last 5 paced beats (figure 1). The slope calculation is based on the least square regression line calculation of the measured points in a selected time window. The begin and end points of this time window are manually adjusted.

To relate the diastolic baseline slope to ouabain-induced delayed afterdepolarizations and triggered arrhythmias, the following parameters were quantified: 1) absolute value of the slope during pacing, 2) occurrence of delayed afterdepolarizations directly post-pacing, 3) the amplitude of the delayed afterdepolarization, 4) induction, number and coupling interval of ectopic beats, and 5) induction of ventricular tachycardia. The presence of a delayed afterdepolarization post-pacing was defined as the occurrence of a depolarizing afterpotential with an ascending and a descending limb, that begins after the complete repolarization (phase 3 ) of the last paced monophasic action potential. ${ }^{2}$ In some cases the delayed afterdepolarization follows an initial phase of hyperpolarization. ${ }^{2}$ This hyperpolarization was excluded in the analyses of delayed afterdepolarization and in the calculation of slope, which were both determined with the phase 4 of the preceding action potential as baseline. Analyses of both the amplitude and coupling interval of delayed afterdepolarizations post-pacing were performed conform methods described elsewhere. ${ }^{23}$ Ventricular tachycardia was defined as more than 5 ectopic beats, having 


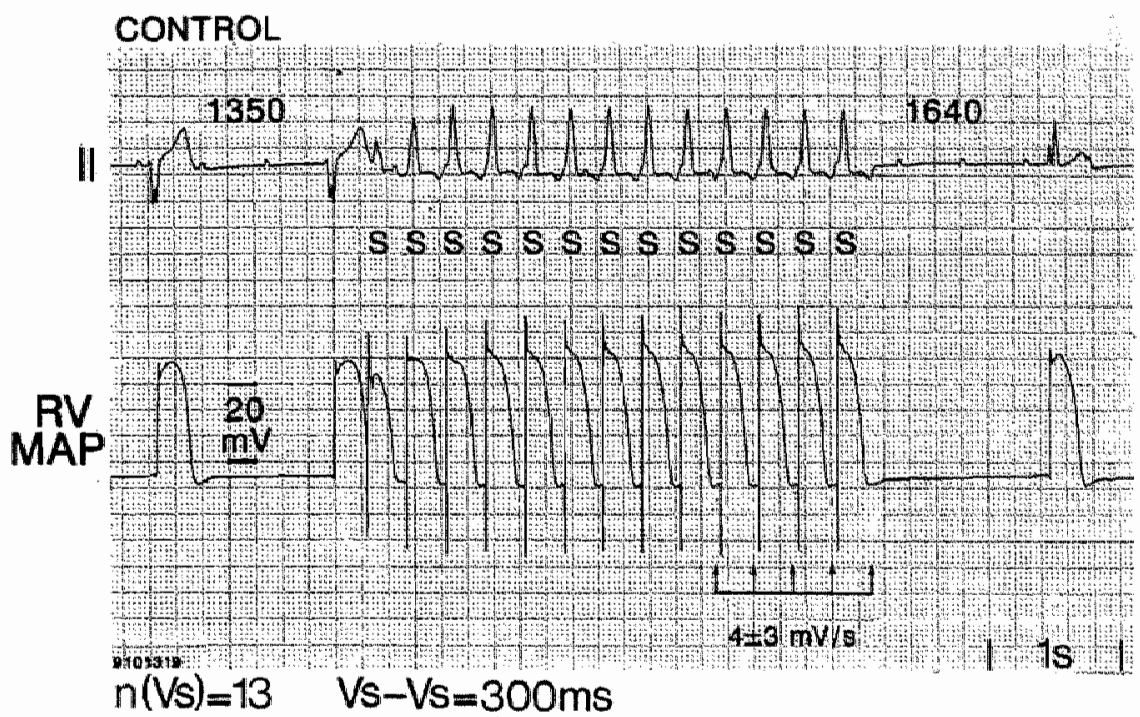

Figure 2: Pacing during control circumstances reveals a flat slope during pacing and no induction of delayed afterdepolarizations (DAD) and ectopic beats (EB) post-pacing .

In this figure, ECG lead II and the right ventricular monophasic action potential recording are shown. Under control, the idioventricular rhythm is interrupted by the start of a pacing train, consisting of 13 stimuli with an interstimulus interval of $300 \mathrm{~ms}$. The diastolic slope at the end of this pacing train remains almost flat (mean: $4 \pm 3 \mathrm{mV} / \mathrm{s}$ ) and there are no DADs induced post-pacing. The first post-pacing interval exceeds the prepacing interval (overdrive-suppression).

a coupling interval shorter than the last spontaneous prepacing idioventricular interval. ${ }^{28}$ To ensure accuracy of our analyses and guarantee uniform interpretation of the results, all experiments were analyzed twice. One of these analyses was done by an independent observer without knowledge of the experimental circumstances.

\section{Statistics}

All data are presented as mean \pm standard deviation (SD). Analysis of variance (ANOVA) followed by Bonferroni's $t$-test (for more than 2 group analysis), two-tailed Student's $t$-test for unpaired events (in case of two group comparison) and Chi-square were used to determine statistical significance. 


\section{Results}

\section{Experiments during chronic atrioventricular-block}

\section{Control versus ouabain}

An almost flat slope was present during control pacing (figure 2): the mean calculated value was $2 \pm 2 \mathrm{mV} / \mathrm{s}$, and delayed afterdepolarizations were induced in $6 \%$ of the pacing trains (table 1). These delayed afterdepolarizations had a mean amplitude of $0.4 \pm 0.1 \mathrm{mV}$. The post-pacing incidence of ectopic beats was $18 \%$, with a mean number of $2 \pm 1$ and a mean coupling interval of $517 \pm 186 \mathrm{~ms}$. Ventricular tachycardias were not induced. Ouabain administration resulted in a non-significant decrease in the spontaneous idioventricular cycle length (from $1432 \pm 377 \mathrm{~ms}$ to $1343 \pm 229 \mathrm{~ms}$ ), and a significant decrease in monophasic action potential amplitude $(32 \pm 13 \mathrm{mV}$ versus $26 \pm 13 \mathrm{mV}, \mathrm{p}<0.05)$. Slope increased to $26 \pm 14 \mathrm{mV} / \mathrm{s}(\mathrm{p}<0.05)$, and there was an increase in incidence and amplitude of delayed afterdepolarizations ( $74 \%$ and $1.0 \pm 0.6 \mathrm{mV}, \mathrm{p}<0.05$ for both, figure 3 ).

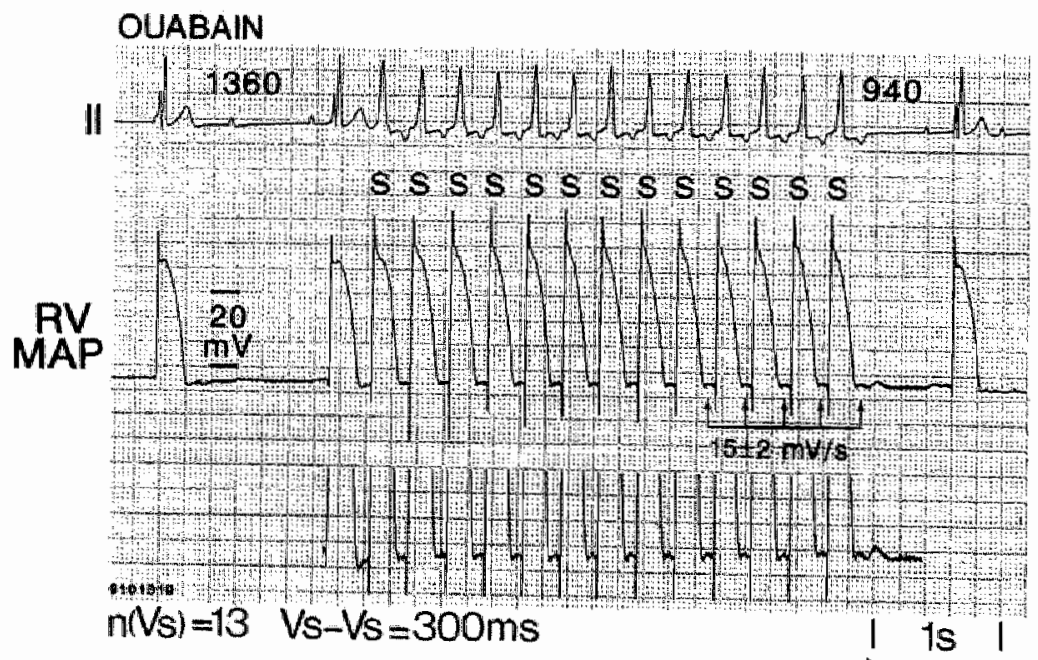

Figure 3: Induction of delayed afterdepolarizations (DAD) during and after pacing during ouabain intoxication.

The composition of this figure is identical to figure 2. After ouabain, the same pacing train in the dog that is represented in figure 2, results in an increase in baseline slope (mean: $15 \pm 2 \mathrm{mV} / \mathrm{s}$ ) and in the occurrence of two DADs and an ectopic beat post-pacing : the coupling interval of the first beat post-pacing is smaller than the prepacing cycle length of the idioventricular rhythm. The presence of the DADs and the increased slope during pacing are especially clear in the enlarged panel, which is shown under the right ventricular monophasic action potential (MAP) recording. 
Table 1: Slope, incidence and amplitude of delayed afterdepolarizations (DADs) and incidence of ectopic ventricular beats and ventricular tachycardia (VT) under the different circumstances studied

\begin{tabular}{lllll}
\hline & Control & Ouabain & Lidocaine & $\begin{array}{l}\text { Washout } \\
\text { Lidocaine }\end{array}$ \\
\hline Slope, mV/s & $2 \pm 2$ & $26 \pm 14^{*}$ & $4 \pm 2 \dagger$ & $24 \pm 7 \ddagger$ \\
DAD, \% & 6 & $74^{*}$ & $57 \dagger$ & $77 \ddagger$ \\
Amplitude DAD, mV & $0.4 \pm 0.1$ & $1.0 \pm 0.6^{*}$ & $0.7 \pm 0.4 \uparrow$ & $1.1 \pm 0.2 \ddagger$ \\
Ectopic beat, \% & 18 & $85^{*}$ & $64 \dagger$ & $100 \ddagger$ \\
VT, \% & 0 & $33^{*}$ & $0 \dagger$ & $55 \ddagger$ \\
\hline
\end{tabular}

${ }^{*} \mathrm{p}<0.05$, ouabain compared with control.

$\dagger \mathrm{p}<0.05$, lidocaine compared with ouabain.

$\$ \mathrm{p}<0.05$, washout lidocaine compared with lidocaine.

Furthermore, pacing increased the incidence of ectopic beats $(85 \%, \mathrm{p}<0.05)$ and ventricular tachycardia ( $33 \%, \mathrm{p}<0.05$, table 1). An example of the induction of ventricular tachycardia is given in figure 4, panel 1 . The number of ectopic beats increased to $23 \pm 47$, while their coupling interval decreased to $481 \pm 282 \mathrm{~ms}$. Both were not significantly different from control values.

\section{Lidocaine and lidocaine wash-out measurements}

Lidocaine decreased the monophasic action potential amplitude to $18 \pm 7 \mathrm{mV}(\mathrm{p}<0.05)$. However during the wash-out period of lidocaine the amplitude rose to $24 \pm 3 \mathrm{mV}$ $(\mathrm{p}<0.05)$. Lidocaine increased the pre-pacing idioventricular cycle length to $1861 \pm 439$ $\mathrm{ms}(\mathrm{p}<0.05$ ) which reversed during wash-out.

Lidocaine decreased the diastolic slope to $4 \pm 2 \mathrm{mV} / \mathrm{s}(\mathrm{p}<0.05)$, reduced the incidence of delayed afterdepolarizations to $57 \%(\mathrm{p}<0.05)$, reduced their mean amplitude to $0.7 \pm 0.4$ $\mathrm{mV}(\mathrm{p}<0.05)$ and completely prevented the occurrence of ventricular tachycardia ( $p<0.05$, figure 4 , panel 2 and table 1 ). The incidence of ectopic beats decreased to $64 \%$ ( $p<0.05$, compared to ouabain), the number of induced beats to $2 \pm 2$, with an increase in the coupling interval to $802 \pm 292 \mathrm{~ms}(\mathrm{p}<0.05)$.

Following wash-out of lidocaine, all parameters increased significantly: 1) slope to $24 \pm 7$ $\mathrm{mV} / \mathrm{s}, 2$ ) induced ventricular tachycardias to $55 \%, 3$ ) delayed afterdepolarizations to $77 \%$ 

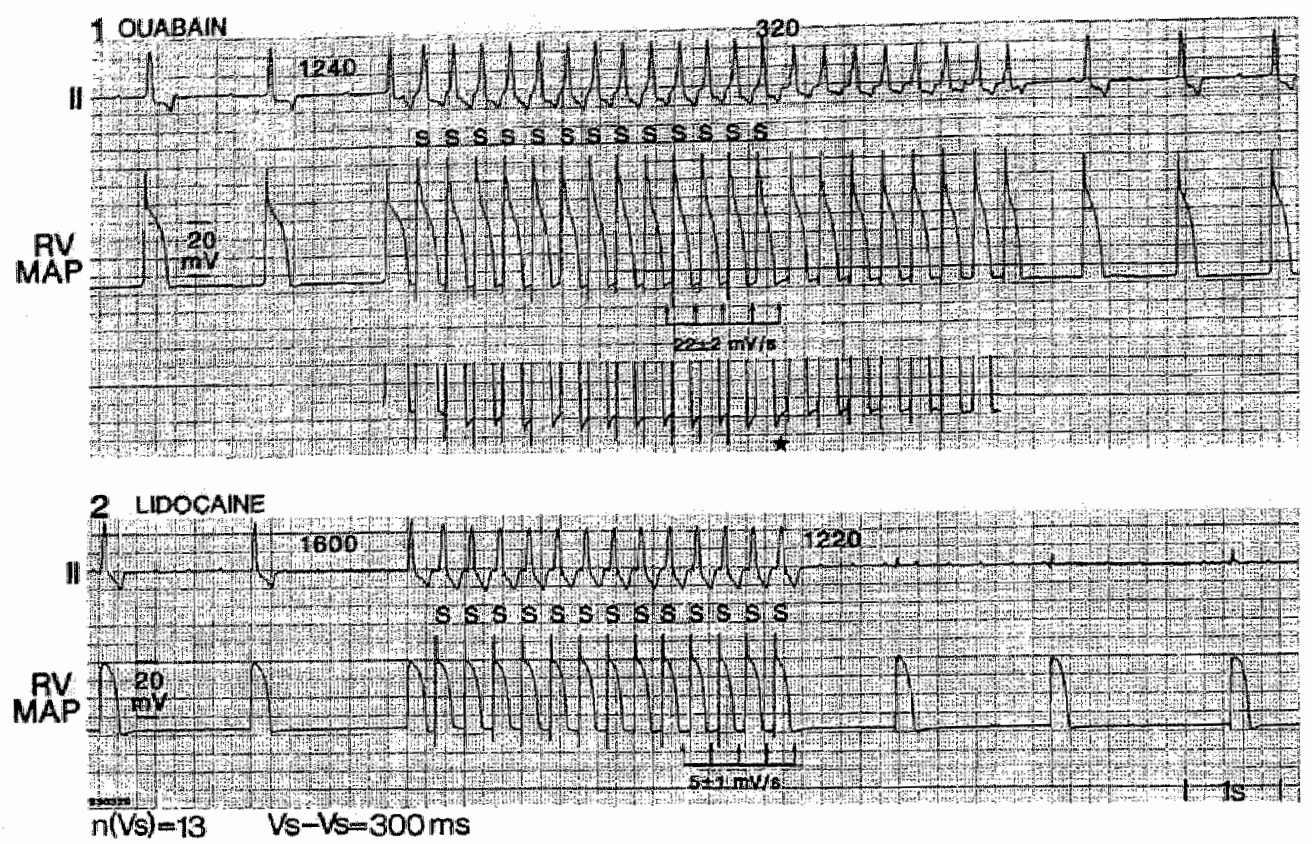

Figure 4: Prevention of induction of a ventricular tachycardia (VT) by lidocaine in the presence of ouabain.

The configuration of this figure is identical to figure 1. Panel 1: Pacing during ouabain with 13 stimuli and an interstimulus interval of $300 \mathrm{~ms}$ results in the induction of VT. During pacing the delayed afterdepolarizations (DADs) are visible in the MAP-recording. The arrows point to the 5 last paced complexes, of which the mean diastolic slope is represented. The asterisk in the enlarged panel indicates the DAD that triggers the first spontaneous beat. During VT, slope declines and VT terminates. Panel 2 : After lidocaine, repetition of the same pacing train, does not result in VT. The slope remains flat during pacing and there are no DADs visible during or after pacing.

with a mean delayed afterdepolarization-amplitude of $1.1 \pm 0.2 \mathrm{mV}$, and 4) incidence of ectopic beats to $100 \%$ (table 1 ).

\section{Interstimulus interval}

During control, the measured parameters did not differ when the two interstimulus intervals were compared in the 5 dogs in which both protocols were performed. The control slope was $2 \pm \rrbracket \mathrm{mV} / \mathrm{s}$ and no delayed afterdepolarizations or ventricular tachycardias were induced in these dogs. 

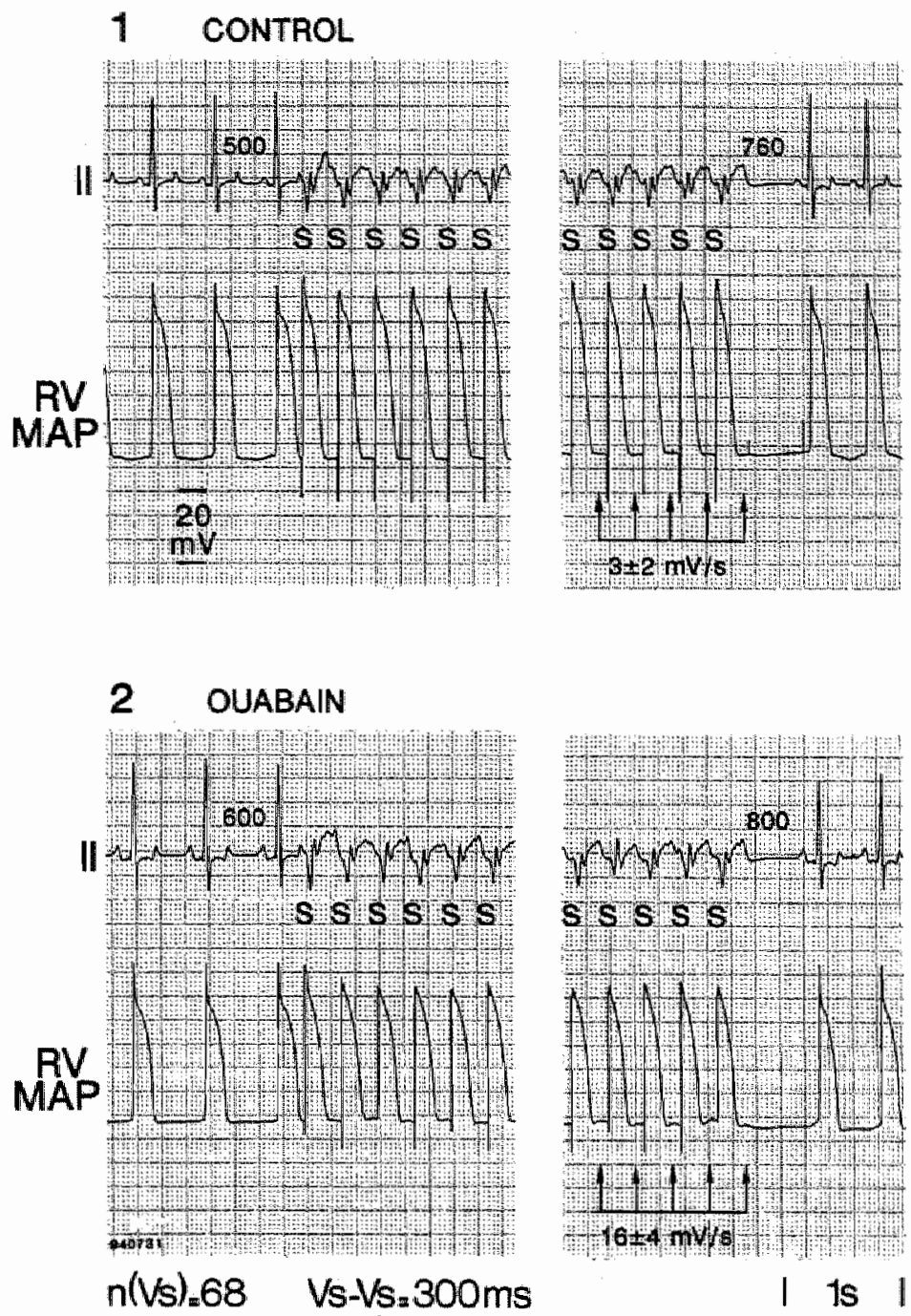

Figure 5: Increased slope and delayed afterdepolarization (DAD) induction after ouabain in dogs with normally conducted sinus rhythm.

Identical composition as figure 1. In the left part of each panel, the start of pacing is visible, while in the right part the end of pacing can be seen.

Panel 1: Pacing consisted of 68 stimuli with an interstimulus interval of 300 ms. During control circumstances, the diastolic slope remains flat and there are no DADs induced.

Panel 2: After ouabain administration, the same pacing train results in an increased slope and a DAD post-pacing. 
During ouabain, the slope, the incidence of delayed afterdepolarizations and ventricular tachycardias increased more markedly after pacing with an interstimulus interval of 300 ms (table 2). Also, the incidence of the ectopic beats after a $400 \mathrm{~ms}$ pacing train was lower, and their coupling interval was longer than after a $300 \mathrm{~ms}$ pacing train, but this did not reach statistical significance. However, the amplitude of induced delayed afterdepolarizations was higher after the $400 \mathrm{~ms}$ stimulation train.

\section{Experiments during sinus rhythm}

Also during sinus rhythm, the slope remained flat $(3 \pm 1 \mathrm{mV} / \mathrm{s})$ during control circumstances (example in figure 5, panel 1). There were no delayed afterdepolarizations induced. After ouabain, both these parameters increased significantly, slope to $17 \pm 4$ $\mathrm{mV} / \mathrm{s}$ and delayed afterdepolarization incidence to $84 \%$ (example in figure 5, panel 2). The delayed afterdepolarization coupling interval and amplitude were $241 \pm 13 \mathrm{~ms}$ and $1.0 \pm 0.5 \mathrm{mV}$ respectively. The sinus cycle length was $497 \pm 12 \mathrm{~ms}$ which slightly decreased after ouabain administration to $460 \pm 50 \mathrm{~ms}$. monophasic action potential amplitude showed a non-significant decrease after ouabain $(34 \pm 20 \mathrm{mV}$ vs $27 \pm 10 \mathrm{mV})$.

Table 2: Relation between interstimulus interval and the different parameters studied during ouabain intoxication

\begin{tabular}{lcc} 
& \multicolumn{3}{c}{$\mathbf{3 0 0}$} & $\mathbf{V s}-\mathbf{V s}, \mathbf{m s}$ & $\mathbf{4 0 0}$ \\
\hline Slope, mV/s & $20 \pm 10$ & $13 \pm 8^{*}$ \\
DADs, \% & 80 & $36^{*}$ \\
Amplitude DAD, mV & $0.7 \pm 0.4$ & $1.1 \pm 0.2^{*}$ \\
Ectopic beats, \% & 73 & 50 \\
VT, \% & 80 & $21^{*}$ \\
Cl ectopic beat, ms & $695 \pm 415$ & $924 \pm 287$ \\
\hline
\end{tabular}

$\mathrm{CI}$ : coupling interval, DAD; delayed afterdepolarization, VT ventricular tachycardia.

* $p<0.05$, interstimulus interval $(\mathrm{V} s \mathrm{~s}$ ) of 400 milliseconds compared with the interstimulus interval of 300 milliseconds. Values are for 5 dogs that were included in both pacing protocols. 


\section{Discussion}

The results of this study indicate that the diastolic slope at the end of a pacing train can 1) be a marker for the ability to induce delayed afterdepolarization-related triggered arrhythmias, 2) possibly be used as a diagnostic tool to identify delayed afterdepolarization-dependent triggered arrhythmias, and 3) be of value to quantitatively assess the effect of (pharmacological) interventions on delayed afterdepolarization-dependent triggered arrhythmias.

\section{Mechanism of arrhythmias during ouabain intoxication}

Partial inhibition of the $\mathrm{Na}^{+} / \mathrm{K}^{+}$pump by ouabain, causes an increase in $\left[\mathrm{Na}^{+}\right] \mathrm{i}$ concentration, that results in an increased $\left[\mathrm{Ca}^{2+}\right] \mathrm{i} .{ }^{3,30,31}$ This intracellular calcium overload causes the sarcoplasmic reticulum to release $\mathrm{Ca}^{2+}$ in the cytosol, eliciting a transient inward current $\left(\mathrm{Iti}_{\mathrm{ti}}\right)^{3,30-33}$ which is responsible for delayed afterdepolarizations. Proposed mechanisms underlying the $\mathrm{I}_{\mathrm{t} i}$ are a calcium-activated non-specific channel and the $\mathrm{Na}^{+} / \mathrm{Ca}^{2+}$ exchanger. ${ }^{32,33}$

\section{Identification of delayed afterdepolarization-dependent triggered activity}

The identification of delayed afterdepolarization-related triggered arrhythmias in patients is difficult, because most diagnostic methods are indirect, or not available for clinical use. Indirect diagnostic features that have been used include: 1) the behavior of delayed afterdepolarization-dependent atrial and ventricular tachycardias in relation to pacing, ${ }^{9-11,16}$ and 2) termination of delayed afterdepolarization-dependent arrhythmias with specific pharmacological probes. ${ }^{13-16,34-36}$ A more direct method that has been used in the intact heart, could be the registration of afterdepolarizations by monophasic action potential signals. ${ }^{16-20}$ However, these recordings should be interpreted with caution, ${ }^{21,22}$ since monophasic action potential recordings are not completely identical to transmembrane measurements. Moreover, the monophasic action potential technique still encounters some practical problems, such as 1) monophasic action potential stability, 2) artifact registration, and 3) selection of the recording site with regard to site of delayed afterdepolarization origin. To minimize the influence of these problems in registering delayed afterdepolarizations, the following criteria should, to our opinion, be fulfilled: 1) monophasic action potential catheters should be placed by experts, to record a stable monophasic action potential with an appropriate shape, 2) the equipment used to record and analyze monophasic action potential signals has to be of high quality, and 3) "delayed afterdepolarizations" should be suppressed by an intervention. 


\section{Model}

The model used in this study has previously been extensively tested and used in our laboratory in conscious dogs. ${ }^{19,28,34,37}$ In order to introduce the monophasic action potential catheter, general anesthesia was used. Otherwise the model remained the same. Pacing during progressive ouabain intoxication eventually leads to 1) the occurrence of ectopic beats, with 2) shortening of their coupling interval, and 3) the induction of ventricular tachycardia. In these experiments however, we did not wait till ventricular tachycardia was induced, which allowed us to study the relation between the diastolic slope and the occurrence of delayed afterdepolarizations, the induction of ectopic beats and ventricular tachycardia.

\section{The behavior of the slope under different circumstances}

The recognition of delayed afterdepolarization-dependent triggered arrhythmias without the necessity to induce sustained arrhythmias can be an important advantage when using the monophasic action potential technique in clinical practice. It is known that pacing can provoke delayed afterdepolarizations under specific conditions, because delayed afterdepolarizations are rate dependent. $4,5,17,18,23,24,28,34,37$ As described in vitro during ouabain, the slope in between stimulated beats becomes progressively more steep and the last paced beat is frequently followed by a subthreshold delayed afterdepolarization or a triggered action potential. ${ }^{4,5,17-20,23,24}$ We have studied this relation in atrioventricular block dogs. In this way, interference of the conducted sinus beats on ventricular arrhythmias is excluded. ${ }^{25}$ However, to ensure the applicability of the diastolic slope during the much faster sinus rate, the experiments were also performed in dogs with normal atrioventricular-conduction.

Our data demonstrate that in the intact globally intoxicated heart, the slope in between paced stimuli might represent the presence of delayed afterdepolarizations. During control circumstances, the slope is relatively flat although a small increase was observed during pacing. This sometimes resulted in the induction of delayed afterdepolarizations and ectopic beats, but never in the generation of a series of triggered beats or ventricular tachycardia. In conscious atrioventricular-block dogs with biventricular hypertrophy, the induction of these ectopic beats by pacing has been described previously. ${ }^{37}$

During ouabain, pacing resulted in a markedly increased slope which was accompanied by a higher incidence of delayed afterdepolarizations, ectopic beats and ventricular tachycardia. The difference in the occurrence of ventricular ectopy and occurrence of delayed afterdepolarizations ( $85 \%$ vs $74 \%$ ) might be explained by a discrepancy between 
monophasic action potential recording site and site of origin of the delayed afterdepolarization. This phenomenon will be more intensively studied in future research.

Lidocaine is known to suppress delayed afterdepolarizations and related arrhythmias in vitro $^{23,38}$ and in the intact heart. ${ }^{36}$ Although it is not a delayed afterdepolarization-specific drug, we have used lidocaine because of its practical advances (relatively short half-life, no complications). Lidocaine completely suppressed ventricular tachycardia, which was accompanied by a decrease in slope, incidence of delayed afterdepolarizations and ectopic beats. These changes disappeared after wash-out, supporting our hypothesis that the slope is a useful indicator for delayed afterdepolarizations and triggered arrhythmias. The decreased monophasic action potential amplitude during lidocaine might be related to negative inotropic effects considering the increase of monophasic action potential amplitude following lidocaine wash-out. ${ }^{39}$

In another series of experiments in which the behavior of the slope during ventricular tachycardia was studied, we have used ryanodine as specific pharmacological probe. Thirteen minutes after ryanodine $(10 \mu \mathrm{g} / \mathrm{kg} / 10$ minutes $)$, all tachycardias terminated $(n=5)$, which was accompanied by a significant decrease in slope (chapter 4$)$.

\section{Influence of paced cycle length}

If the diastolic slope is representative for delayed afterdepolarizations and triggered arrhythmias, the slope should be influenced by the interstimulus interval. $^{4,5,10,12,17,18,23,24}$ Under control circumstances no difference was seen in slope at different pacing rates. After ouabain, the slope behaved in accordance with our hypothesis being less pronounced at slower pacing rates. A relation was found between pacing rate and incidence of delayed afterdepolarizations, the occurrence of ectopic beats, and the induction of ventricular tachycardia. The only discrepancy is the amplitude of the first delayed afterdepolarization post-pacing, which is higher after the $400 \mathrm{~ms}$ stimulation train. This is however, a difference caused by analysis, since a direct comparison of the 2 dogs that showed delayed afterdepolarizations after stimulating with both frequencies, revealed a higher delayed afterdepolarization amplitude after the $300 \mathrm{~ms}$ stimulation trains.

Another possible explanation for a difference in delayed afterdepolarization amplitude, could have been the occurrence of 2 delayed afterdepolarizations after $300 \mathrm{~ms}$ pacing and 1 delayed afterdepolarization after pacing with a $400 \mathrm{~ms}$ interstimulus interval. Although the occurrence of 2 delayed afterdepolarizations after the $300 \mathrm{~ms}$ pacing trains 
was seen twice in our total group of experiments, this phenomenon was not observed in this series of 5 dogs.

\section{Limitations}

In this study, the slope was measured in a randomly placed right ventricular monophasic action potential recording, without considering the relevance of the place of origin of the delayed afterdepolarizations in relation to the registration site. Pacing was always performed from the monophasic action potential catheter which also registered the delayed afterdepolarizations. A stable monophasic action potential signal with a certain amplitude was in this study considered to be of greater importance than a search for specific sites in which delayed afterdepolarizations occur. ${ }^{40}$ We used a globally intoxicated dog heart and do not know to what extent our registration of delayed afterdepolarizations is a true manifestation of delayed afterdepolarizations at the site of origin. Other experiments are necessary to validate our approach during pathological circumstances of calcium overload, possibly occurring in more localized areas. It should also be evaluated in patients with idiopathic ventricular tachycardia, and atrial tachycardia. ${ }^{16}$

In summary, the diastolic slope of the monophasic action potential recording during pacing might represent a parameter to evaluate the presence of delayed afterdepolarizations and triggered activity in the intact heart. This could be demonstrated by measuring the diastolic slope during a simple pacing train in circumstances suspect for the occurrence of delayed afterdepolarizations, such as during ouabain intoxication. This suggests that the slope can possibly be used as a quantitative marker to evaluate interventions on delayed afterdepolarization-dependent arrhythmias in both the animal heart and in humans.

\section{Acknowledgments}

This study was supported by a grant from the Wynand N. Pon Foundation, The Netherlands. We thank the Bakken Research Center, Maastricht, The Netherlands, for supplying the electrodes and the University of Limburg Technical Service (Leon Dohmen, BSc) for building and maintaining the equipment.

\section{References}

1. Hoffman BF Rosen MR. Cellular mechanisms for cardiac arthythmias. Circ Res 1981; 49: 1-15.

2. Cranefield PF, Aronson R. Cardiac arrhythmias: The role of triggered activity and other mechanisms. Mt Kisco, New York, Futura publishing Co, 1988. 
3. Kass RS, Lederer WJ, Tsien RW, Weingart R. Role of calcium ions in transient inward currents and aftercontractions induced by strophantidin in cardiac purkinje fibers. J Physiol 1978; $28: 187.208$.

4. Rosen MR, Gel band H, Merker C, Hoffman BF. Mechanisms of digitalis toxicity. Effects of ouabain on phase four of canine purkinje fiber transmembrane potentials. Circulation 1973; 47 : 681-689.

5. Ferrier GR, Saunders JH, Mendez C. A cellular mechanism for the generation of ventricular rrhythmias by acetylstrophanthidin. Circ Res 1973; 32: 600-609.

6. Rosen MR, Visch C, Hoffman BF, Danilo P, Lovelace DE, Knoebel SB. Can accellerated atrioventricular junctional escape beats be explained by delayed afterdepolarizations? Am J Cardial 1980; 45: $1272-1284$.

7. Gorgels APM, Vos MA, Smeets JLRM, Kriek R, Brugada P, Wellens HJJ. Delayed afterdepolarizations and atrial and ventricular arrhythmias. In: Rosen MR, Janse MJ, Wit AL, eds. Cardiac electrophysiology: a textbook. New York, Mount Kisco, Futura Publishing Co., 1990; 341-354.

8. Rosen MR, Reder RF. Does triggered activity have a role in the genesis of cardiac arrhythmias? $A n m$ Intern Med 1981; 94: 794-801.

9. Bhandari AK, Hong RA, Rhahimtoola SH. Triggered activity as a mechanism of recurrent ventricular tachycardia. Br Heart $J 1988$; 59: 501-505.

10. Rosen MR. Is the response to programmed electrical stimulation diagnostic of mechanisms for arrhythmias? Circulation 1986; 73: (suppl II) 18-27.

11. Wellens HJJ, Brugada P, Vanagt EJDM, Ross DL, Bär FW. New studies with triggered automaticity.In: Harrison DC, ed. Cardiac Arrhythmias adecade of progress. Boston, G.K. Hall Medical Publishers, $1981 ; 601-610$.

12. Brugada $\mathrm{P}$, Wellens $\mathrm{HJJ}$. The role of triggered activity in clinical ventricular arrhythmias. $P A C E$ 1984; 7:. 260-271.

13. Lerman $B B$, Belardinelli $\mathbb{L}$, West A, Berne RM, DiMarca JP. Adenosine sensitive ventricular tachycardia: evidence suggesting cyclic AMP-mediated triggered activity. Circulation 1986; 74 : 270- 280 .

14. Sung RJ, Shapiro WA, Shen EN, Morady F. Effects of verapamil on ventricular tachycardias possibly caused by reentry, automaticity, and triggered activity. J Clin Invest 1983; 72: 350-360.

15. Gorgels APM, Vos MA, Leunissen JDM, Dijkman B, JLRM Smeets, Wellens HJJ. Flunarizine as a specific drug to identify triggered activity based on delayed afterdepolarizations. In: Josephson

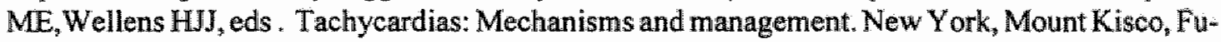
tura Publising Co Inc, 1993; 87-97.

16. Chen SA, Chiang CE, Yang CJ, Cheng CC, Wu TJ, Wang SP, Chiang BN, Chang MS, Sustained atrial tachycardia in adult patients. Electrophysiological characteristics, pharmacological response, possible mechanisms, and effects of radiofrequency ablation. Circulation 1994; 90: 1262-1278.

17. Priori $S G$, Mantica M, Schwartz PJ. Delayed afterdepolarizations elicited in vivo by left stellate ganglion stimulation. Circulation 1988; 78: 178-185.

18. Furukawa T, Kimura S, Castellanos A, Bassett AL, Myerburg RJ. In vivo induction of focal triggered ventricular arrhythmias and responses to overdrive pacing in the canine heart. Circulation $1990 ; 82: 549-559$.

19. Vos MA, Fazekas T, Gorgels APM, Leunissen HDM, Wellens HJJ. The action of MgSO 4 differs from moricizine and verapamil on ouabain-induced ventricular tachycardia in normomagnesemic conscious dogs. I Cardiovasc Pharmacol 1994; $23: 252-258$.

20. Patterson $\mathrm{E}_{\mathrm{y}}$ Szabo B, Scherlag BJ, Lazzara R. Early and delayed afterdepolarizations associated with cesium chloride induced arrhythmias in the dog. J Cardiovasc Pharmacol 1990; 15: 323-331. 
21. Olsson SB, Blomström $\mathrm{P}$, Blomström-Lundquist $\mathrm{C}$, Wohlfart $\mathrm{B}$. Endocardial monophasic action potentials, Correlations with intracellular electrical activity. Am NY Acad Sci 1990; 601: 119-127.

22. Franz MR, BurkhoffD, Yue DT, Sagawa K. Mechanically induced action potential changes and arthythmias in isolated and in situ canine hearts. Cardiovasc Res $1989 ; 23: 213-223$.

23. Rosen MR, Danilo P. Effects of tetrodotoxin, lidocaine, verapamil, and AHR-2666 on ouabain induced delayed afterdepolarizations in canine Purkinje fibers. Circ Res 1980; 46: 117-124.

24. Felzen $B$, Lotan $R$, Binah $O$. Inter-species wariations in myocardial responsiveness to cardiac glycosides: Possible relations to the thyroid status. I Mol Cell Cardiol 1989; 21: 165-174.

25. Steiner $\mathrm{CH}, \mathrm{K}$ ovalic ATHW. A simple technique for production of chronic complete heart block in dogs. J Appl Physiol 1968; 25:631-632.

26. Franz MR, Chin MC, Sharkey HR, Griffin JC, Scheinman MM. A new single catheter technique for simultaneous measurement of action potential duration and refractory period in vivo. $J \mathrm{Am} \mathrm{Coll}$ Cardiol 1990; 16: 878-886.

27. Franz MR. Method and theory of monophasic action potential recording. Prog Cardiovasc Dis $1991 ; 33: 347-368$.

28. Vos MA, Gorgels APM, Leunissen HDM, wan Deursen RTAM, Wellens HJJ. Significance of the number of stimulli to initiate ouabain-induced arrhythmias in the intact heart. Circ Res $1991 ; 68 ; 38$ 44.

29. Rhee HM, Dutta S, Marks BH. Cardiac NaK ATP-ase activity during positive inotropic and toxic actions of ouabain. Eur J Pharmacol 1976; 37: 141-153.

30. Rosen MR. Dellyyed afterdepolarizations induced by digitall is. In: Rosen MR, Janse MJ, Wit AL, eds. Cardiac electrophysiology: a textbook. New York, Mount Kisco, Futura, 1990; 271-281.

31. Smith TW. Digitalis. Mechanisms of action and clinical use. N Engl J Med 1988; 318: 358-365.

32. Berlin JR, Cannell MB, Lederer WJ. Cellular origins of the transient inward current in cardiac myocytes. Role of fluctuations and waves of elevated intracellular calcium. Circ Res 1989; 65: 115-126.

33. Fedida N, Noble D, Rankin AC, Spindler AJ. The arrhythmogenic transient inward current Iti and related contraction in isolated guinea-pig ventricular myocytes. J Physiol 1987; 392: 523-542.

34. Vos MA, Gorgels APM, Leunissen HDM, Wellens HJJ. Flunarizine allows differentiation between mechanisms of arrhythmias in the intact heart. Circulation 1990; 81:343-349.

35. Le Marec $\mathrm{H}$, Spinelli W, Rosen MR. The effects of doxorubicin on wentricular tachycardia. Circulation 1986; 74: 881-889.

36. Vos MA, Gorgels APM, Leunissen HDM, Korterink W, Wellens HJJ. Discriminative power of drugs to identify specifie arrhythmogenic mechanisms in vivo. Cardiovase Drug Ther 1991; 5 : 402. (abstract)

37. Vos MA, Gorgels APM, De Wit B, Drenth JPH, Van Deursen RTAM, Leun issen JDM, Wellens HJJ. Premature escape beats, a model for triggered activity in the intact heart? Circulation 1990; 82: 213-224.

38. Karagueuzian HS, Katzung BG. Relative inotropic and arrhythmogenic effects of five cardiac steroids in ventricular myocardium: Oscillatory afterpotentials and the role of endogenous catechoUamines. J Pharm Exp Ther 1981; $21: 348-356$.

39. Sheu SS, Lederer WJ. Lidocaine's negative inotropic and antiarrhythmic actions. Circ Res 1985; 57: 578-590.

40. Gorgels APM, De Wit B, Beekman HDM, Dassen WRM, Wellens HJJ. Effects of different modes of stimulation on the morphology of the first QRS-complex following pacing during digitalis induced ventricular tachycardia: observations in the conscious dog with chronic complete AV-block. PACE 1986; 9: 842-859. 


\title{
Chapter 3
}

The dynamic behavior of the diastolic slope of the monophasic action potential can be related to the occurrence and maintenance of delayed afterdepolarization-dependent arrhythmias in the canine heart

\author{
Marieke de Groot \\ Marc Vos \\ Anton Gorgels \\ Jet Leunissen \\ Leon Dohmen \\ Marc Hermans \\ Hein Wellens
}

Abstracts presented at the North American Society of Pacing and Electrophysiology, 1994 (PACE 1994; 17: 121) and 1995 (PACE 1995; 18: 344)

Submitted for publication 


\section{Abstract}

Introduction: We have described the diastolic slope of the monophasic action potential recording at the end of pacing as a marker for delayed afterdepolarization-dependent arrhythmias. In the present study 1) the behavior of the slope at different time points during a pacing train was quantified and related to the arrhythmic outcome (group A) and 2) termination of delayed afterdepolarization-dependent ventricular tachycardia was related to changes in the slope (group B).

Methods and results: In dogs with atrioventricular-block, a monophasic action potential was recorded during 1) ventricular pacing, before and after ouabain administration (A) and 2) 6 spontaneous and 6 lidocaine induced ventricular tachycardia terminations (B). A: During control, the slope at the end of the pacing train was $5 \pm 3 \mathrm{mV} / \mathrm{s}$ (mean $\pm \mathrm{SD}$ ), independent of the pacing duration. During ouabain, this increased to $20 \pm 13 \mathrm{mV} / \mathrm{s}$ $(p<0.05)$, varying with the duration of pacing. Slope was steeper after pacing for 4 seconds compared to 20 seconds $(26 \pm 12 \mathrm{mV} / \mathrm{s}$ vs. $16 \pm 13 \mathrm{mV} / \mathrm{s}, \mathrm{p}<0.05$ ) which corresponded with more frequent ventricular tachycardia induction.

$B$ : In spontaneously terminating ventricular tachycardias, cycle length increased from $353 \pm 54 \mathrm{~ms}$ at the start to $434 \pm 78 \mathrm{~ms}(\mathrm{p}<0.05)$ before ventricular tachycardia termination. This corresponded with a decreasing slope from $19 \pm 10 \mathrm{mV} / \mathrm{s}$ to $6 \pm 5 \mathrm{mV} / \mathrm{s}(\mathrm{p}<0.05)$. In lidocaine induced ventricular tachycardia termination, the cycle length and the slope showed an identical behavior.

Conclusions: There is a dynamic variation in the steepness of the diastolic depolarization during pacing, which depends on the duration of pacing and predicts arrhythmogenic outcome. Furthermore, a decrease in slope during delayed afterdepolarization-dependent ventricular tachycardia can be used to predict ventricular tachycardia termination.

\section{Introduction}

Triggered activity resulting from delayed afterdepolarizations has been extensively investigated in isolated tissue preparations during cardiac glycoside intoxication. ${ }^{1-4}$ Other circumstances in which delayed afterdepolarizations occur are after catecholamines ${ }^{5}$ reperfusion, ${ }^{6}$ hypokalemia, ${ }^{7}$ the (sub)acute phase of myocardial infarction, ${ }^{8}$ and in ventricular hypertrophy. ${ }^{9}$ In spite of all this experimental information, clinical identification of delayed afterdepolarizations as the mechanism responsible for initiation and/or perpetuation of arrhythmias remains difficult. A diagnostic method that has demonstrated the involvement of delayed afterdepolarizations in the initiation of cardiac 
glycoside induced ventricular arrhythmias in animals, is the monophasic action potential technique. ${ }^{10-13}$ Recently, ${ }^{12}$ we have demonstrated that the diastolic slope of the monophasic action potential at the end of a pacing train can be used to quantify the ability to initiate delayed afterdepolarization-dependent triggered activity post-pacing. It was the purpose of this study: 1) to determine the exact (dynamic) behavior of the slope (delayed afterdepolarizations) measured at different time points during pacing and to relate this to arrhythmogenic outcome of pacing, and 2) to elucidate whether the slope can also be used to obtain information in relation to ventricular tachycardia behavior: continuation or termination.

\section{Methods}

\section{General}

All experiments were performed in accordance with the "Guiding principles in the care and use of animals" as approved by the American Physiological Society and under the regulations of the Committee for Experiments on Animals of the University of Limburg, Maastricht, The Netherlands.

The studies were performed in 17 anesthetized dogs (mean body weight of $25 \pm 3 \mathrm{~kg}$, table 1) of either sex. Complete atrioventricular-block was made to exclude the interference of conducted sinus beats on ventricular arrhythmias. ${ }^{14}$ During the atrioventricular-block operation an epicardial screw-in electrode (Bakken Research Center, Maastricht, The Netherlands) was placed on the apex of the left ventricle. The experiments were performed $7 \pm 4$ weeks after the atrioventricular-block operation (table 1).

Experiments were performed under anesthesia and sterile conditions. Premedication $(0.2$ $\mathrm{ml} / \mathrm{kg}$ ) was given intramuscularly with a drug mixture containing $10 \mathrm{mg}$ oxycodan $\mathrm{HCl}$, $1 \mathrm{mg}$ acepromazine and $0.5 \mathrm{mg}$ atropine sulfate per $\mathrm{ml}$. An antibiotic ( $1000 \mathrm{mg}$ ampicillin, Pentrexyl ${ }^{\mathrm{R}}$, Bristol Myers Squibb) was given preoperatively (IV) and postoperatively (IM). Anesthesia was induced by sodium pentobarbital ( $20 \mathrm{mg} / \mathrm{kg} \mathrm{IV})$. The dogs were artificially ventilated (Pulmomat respirator, Dräger), through a cuffed endotracheal tube with a mixture of oxygen, nitrous oxide and halothane (vapor concentration $0.5-1.0 \%$ ). Ventilation was adjusted on the basis of continuous monitoring of the carbon dioxide concentration of the expired air. A thermal mattress was used to maintain body temperature around $37^{\circ} \mathrm{C}$ during the experiments.

Stimulation was done with a programmable stimulator having a synchronizing circuit. Six surface ECG leads and a single site monophasic action potential recording were 
simultaneously registered on a monitor and stored on an optical disc. Drugs were administered through a cannula in a cephalic vein.

\section{Recording of monophasic action potentials}

In order to register endocardial monophasic action potential signals, a quadripolar contact electrode ${ }^{15}$ (Steerable Franz ${ }^{\mathrm{TM}}$ Combination Catheter, EP technologies), was placed endocardially in the right ventricle through the femoral or external jugular vein, under fluoroscopic guidance. The monophasic action potential signals were amplified using a custom build DC-coupled differential amplifier with a $20 \mathrm{mV}$ calibration pulse. The offset of this amplifier is variable and can be adjusted to the recorded signal. The monophasic action potential signals are sampled at a rate of $1 \mathrm{kHz}$ per signal. For a detailed description of the methodology, we refer to a previous publication. ${ }^{12}$ In short, the monophasic action potential signals were checked for stability and had a minimally accepted amplitude of $15 \mathrm{mV}$ before the experiment started. ${ }^{12,15}$ Additionally, rigorous attention was paid to record stable diastolic potentials and monophasic action potentials with a constant configuration and shape during control circumstances. ${ }^{12}$ Prior to each pacing protocol, the amplitude of the monophasic action potential signal was checked. If the amplitude was found to be below $15 \mathrm{mV}$, the contact pressure of the catheter tip was slightly increased, while maintaining the position and configuration of the signal. In protocol $\mathrm{B}$, the monophasic action potentials had to have a constant configuration and shape both before and after ventricular tachycardia termination to be included in the analyses. To illustrate the stability of the monophasic action potential recordings, the amplitude and the diastolic slope of the monophasic action potential signals at the start and the end of each quantified experimental period are given in table 1 . These values are obtained during the idioventricular rhythm.

\section{Experimental protocol}

During both protocols, ouabain was administered as a bolus (bolus $45 \pm 5 \mu \mathrm{g} / \mathrm{kg}$ in 1 minute), followed after 10 minutes by continuous infusion (range 0.072 to $0.090 \mu \mathrm{g} / \mathrm{kg}$ per minute). The behavior of the slope during pacing (group A, 9 dogs) was studied during 3 stimulation trains with an interstimulus interval of $300 \mathrm{~ms}$ and a duration of 4 , 10 and 20 seconds $(13,33$, and 68 stimuli). Pacing was performed from the monophasic 
Table 1: Overwiew of the dogs used and the stability of the monophasic action potential recordings

\begin{tabular}{|c|c|c|c|c|c|c|c|c|}
\hline Dog & Weight & AVB & Group A & Group B & $\begin{array}{l}\text { A MAP } \\
\text { start }\end{array}$ & $\begin{array}{l}\text { A MAP } \\
\text { end }\end{array}$ & $\begin{array}{l}\text { Slope } \\
\text { Start }\end{array}$ & $\begin{array}{l}\text { Slope } \\
\text { end }\end{array}$ \\
\hline 1 & 22 & 2 & 1 & & 24 & 17 & 1.5 & 3.6 \\
\hline 2 & 29 & 3 & 1 & & 21 & 17 & 4.1 & 2.7 \\
\hline 3 & 30 & 9 & 1 & & 28 & 24 & 1.9 & 3.4 \\
\hline 4 & 25 & 7 & 1 & & 30 & 22 & 4.0 & 7.6 \\
\hline 5 & 25 & 4 & 1 & & 20 & 15 & 1.2 & 0.3 \\
\hline 6 & 26 & 9 & 1 & 1 & 35 & 30 & 2.7 & 5.3 \\
\hline 7 & 21 & 8 & 1 & 1 & 27 & 25 & 1.5 & 2.9 \\
\hline 8 & 24 & 9 & 1 & 1 & 28 & 26 & 6.2 & 1.7 \\
\hline 9 & 26 & 3 & 1 & 1 & 24 & 19 & 3.9 & 4.8 \\
\hline 10 & 23 & 2 & & 1 & 26 & 22 & 4.9 & 5.9 \\
\hline 11 & 24 & 8 & & 1 & 20 & 18 & 2.3 & 5.2 \\
\hline 12 & 24 & 3 & & 1 & 24 & 20 & 3.3 & 4.3 \\
\hline 13 & 20 & 20 & & 1 & 18 & 15 & 2.1 & 3.2 \\
\hline 14 & 24 & 7 & & $\mathbb{1}$ & 25 & 22 & 1.1 & 3.2 \\
\hline 15 & 23 & 3 & No VT & & & & & \\
\hline 16 & 33 & 8 & No VT & & & & & \\
\hline 17 & 22 & 6 & No VT & & & & & \\
\hline Mean & 25 & 7 & & & 25 & 21 & 3 & 4 \\
\hline SD & 3 & 4 & & & 5 & 4 & 2 & 2 \\
\hline
\end{tabular}

Weight: in kg, AVB: weeks atrioventricular block, A MAP: amplitude of the monophasic action potential signal, Group A: Behavior of slope during pacing, Group B: Behavior of slope during VT and before termination, "Start" and "End" of the quantified experimental period 
action potential catheter with a strength of $1 \mathrm{~mA}$ and a pulse width of $2 \mathrm{~ms}$. This protocol was performed during control and repeated once 15-30 minutes after ouabain. In 6 dogs, the described pacing protocol was expanded with 3 stimulation trains with an interstimulus interval of $400 \mathrm{~ms}$ and identical durations $(10,25$, and 50 stimuli).

In group B, 12 dogs were used of which 4 dogs were also used in group $\mathrm{A}$ (table 1). ventricular tachycardia was induced using the same stimulation protocol. Either the monophasic action potential catheter or the left ventricular epicardial screw electrode was used to stimulate the heart. Pacing from the left ventricular epicardial electrode was done at twice diastolic threshold and a pulse width of $2 \mathrm{~ms}$. Pacing was performed during control, resumed 15 minutes after ouabain, and repeated every 15 minutes until ventricular tachycardia induction. In 3/12 dogs, the combination of ouabain administration and pacing did not result in ventricular tachycardia induction (table 1) within the 2 hours time span which was considered to be appropriate. ${ }^{16}$ In the remaining 9 dogs, 6 spontaneously terminating ventricular tachycardias with a duration of at least 10 seconds and 6 ventricular tachycardias with a duration of more than 5 minutes were induced. The latter ventricular tachycardias were terminated with lidocaine intravenously ( $3 \mathrm{mg} / \mathrm{kg}$ in 2 minutes).

\section{Data analysis}

Using a software program, ${ }^{12}$ the data were analyzed off line. Cycle length of the idioventricular rate, amplitude of the monophasic action potential and duration of the action potential at $100 \%$ repolarization were measured before the start of each pacing train. To relate the diastolic slope to induction, perpetuation and/or termination of ouabain induced ventricular tachycardias, the following parameters were calculated: 1 ) the baseline slope $(\mathrm{mV} / \mathrm{s})$ of the diastolic interval of the monophasic action potential recordings, 2) the presence of delayed afterdepolarizations, 3) induction of ectopic beats and ventricular tachycardia, and 4) ventricular tachycardia cycle length (ms). Slope was calculated as the mean rate of rise (dV/dt) of the ascending $\lim b$ of the delayed afterdepolarization, or as the mean change in diastolic potential in between the paced or tachycardia beats ${ }^{12}$. If hyperpolarization was present, this was excluded in the calculation of the slope. ${ }^{12}$ A delayed afterdepolarization was defined as a depolarizing afterpotential that begins after the complete repolarization. ${ }^{3,12}$ ventricular tachycardia was defined as the occurrence of more than 5 ectopic beats post-pacing, having a coupling interval shorter than the last spontaneous prepacing idioventricular interval. ${ }^{16}$ If the first beat after pacing had a longer coupling interval, it was defined overdrive suppressed. ${ }^{16}$ 


\section{Dynamic behavior of slope during pacing}

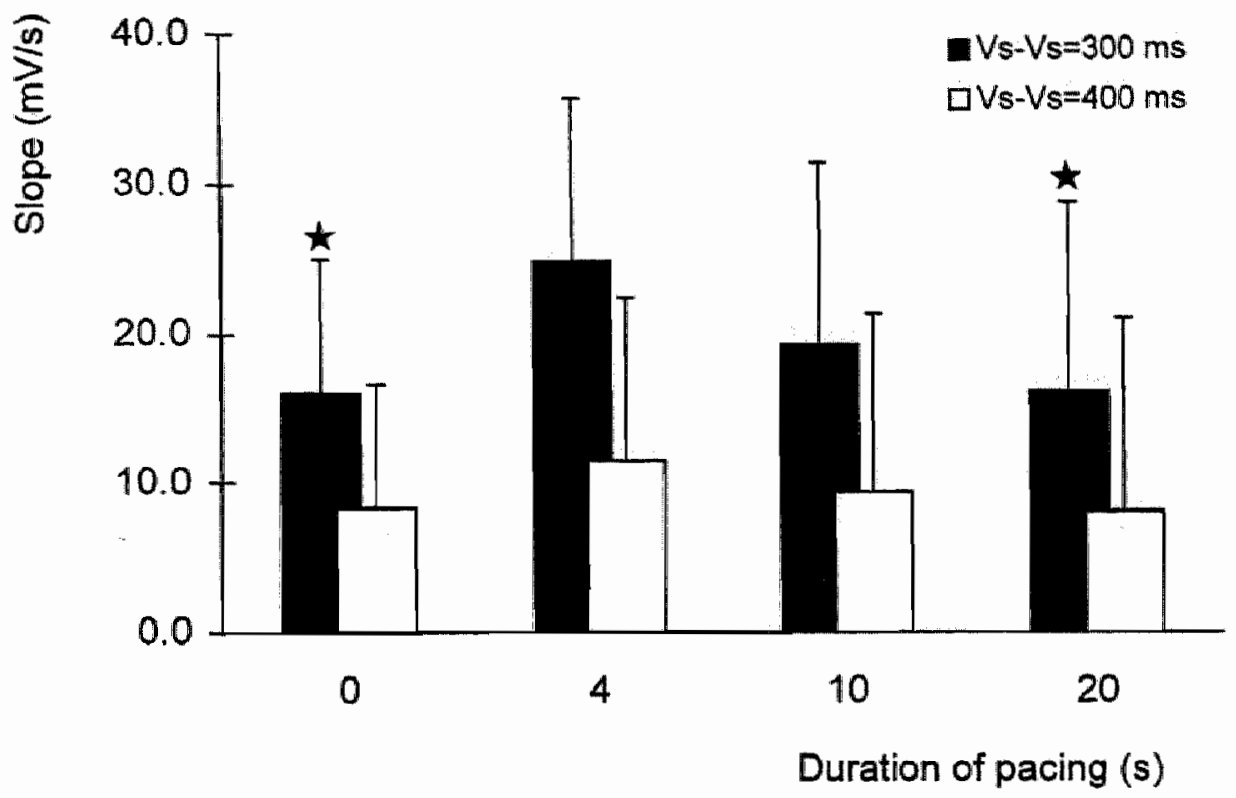

Figure 1: In this bar chart the behavior of the diastolic slope ( $Y$-axis) during pacing measured at different durations is illustrated for both the 300 and the $400 \mathrm{~ms}$ interstimulus interval. At the $X$-axis, the duration of pacing is indicated in seconds. Both paced interstimulus intervals reveal the same pattern of slope behavior during pacing: after an initial increase, slope declines and returns to starting values. In each bar, the number of averaged pacing trains is given. The asterisk ( $\left.{ }^{*}\right)$ indicates significance when compared to a pacing duration of 4 seconds, i.e. beats $9-13$ for the $300 \mathrm{~ms}$. For statistical significance (repeated measures ANOVA), only identical pacing trains were compared.

To analyze the behavior of the slope during pacing and prior to arrhythmia induction (group A), slope was calculated for the first 5 paced beats (1-5), beats 9-13, beats 29-33, and beats $64-68$, when analyzing the $300 \mathrm{~ms}$ pacing protocol. The $400 \mathrm{~ms}$ pacing protocol was analyzed at the same time points, i.e. beats $1-5$, beats $6-10$, beats $21-25$, and beats 46-50.

The behavior of slope during ventricular tachycardia perpetuation and termination (group B) was quantified by calculating the mean slope of 5 beats at different time points. These points were normalized to the duration of the ventricular tachycardia $(0 \%, 25 \%, 50 \%$, $75 \%, 100 \%$ ). Only sustained ventricular tachycardias with a duration of more than 10 
seconds were analyzed. For observations during lidocaine infusion (given after 5 minutes sustained ventricular tachycardia), ventricular tachycardia duration was set at $0 \%$ at the start of the infusion, while at termination ventricular tachycardia duration equals $100 \%$ in both groups. At the same time points ( $0 \%, 25 \%, 50 \%, 75 \%, 100 \%)$, the cycle length of the ventricular tachycardia was determined. Termination of ventricular tachycardia was defined either as complete termination or slowing down of the rhythm to cycle lengths of more than $600 \mathrm{~ms}$.

\section{Statistics}

All data are presented as mean \pm standard deviation (SD). To determine statistical significance, repeated measures analyses of variance (ANOVA) followed by Bonferroni's $t$-test, two-tailed Student's $t$-test for paired events (in case of two group comparison), and Chi-square were used.

\section{Results}

\section{Group A: Behavior of slope during pacing}

Under control conditions, the slope was flat at the beginning $(4 \pm 3 \mathrm{mV} / \mathrm{s}$, mean $\pm \mathrm{SD}$ of all pacing trains) and remained flat to the end of pacing $(5 \pm 3 \mathrm{mV} / \mathrm{s}$, mean $\pm \mathrm{SD}$ of all pacing trains) which was followed by overdrive suppression in $85 \%$ of all pacing trains. Overdrive suppression was most pronounced after pacing for 20 seconds $(3960 \pm 1633$ ms versus $2072 \pm 712 \mathrm{~ms}$ after pacing for 4 seconds, $\mathrm{p}<0.05$ ). The other $15 \%$ of the pacing trains were followed by a single ectopic ventricular beat.

Ouabain administration resulted in an insignificant decrease of the cycle length of the idioventricular rhythm (from $1644 \pm 413$ to $1443 \pm 330 \mathrm{~ms}$ ), a shortening of action potential duration (from $303 \pm 43$ to $273 \pm 39 \mathrm{~ms}, \mathrm{p}<0.05$ ) and a decrease of monophasic action potential amplitude $(26 \pm 5$ versus $22 \pm 5 \mathrm{mV}$, $\mathrm{p}<0.05$, table 1$)$. During ouabain, the slope at the start and at the end of pacing was significantly higher when compared to control ( $16 \pm 9$ and $20 \pm 13 \mathrm{mV} / \mathrm{s}, \mathrm{p}<0.05$, mean $\pm \mathrm{SD}$ of all pacing trains) and now ectopic beats were induced after $89 \%$ of pacing trains ( $p<0.05$ ). Comparing the different durations of pacing, it was noticed that the slope was steeper at the end of the pacing train after pacing for 4 seconds when compared to 20 seconds (respectively $26 \pm 12 \mathrm{mV} / \mathrm{s}$ versus $16 \pm 13$ $\mathrm{mV} / \mathrm{s}, \mathrm{p}<0.05$, table 2 , third column). The increased slope was associated with more frequent induction of ventricular tachycardia (table 2). When all pacing trains were compared at identical time points, the slope increased from $16 \pm 9 \mathrm{mV} / \mathrm{s}$ at beats $1-5$ 

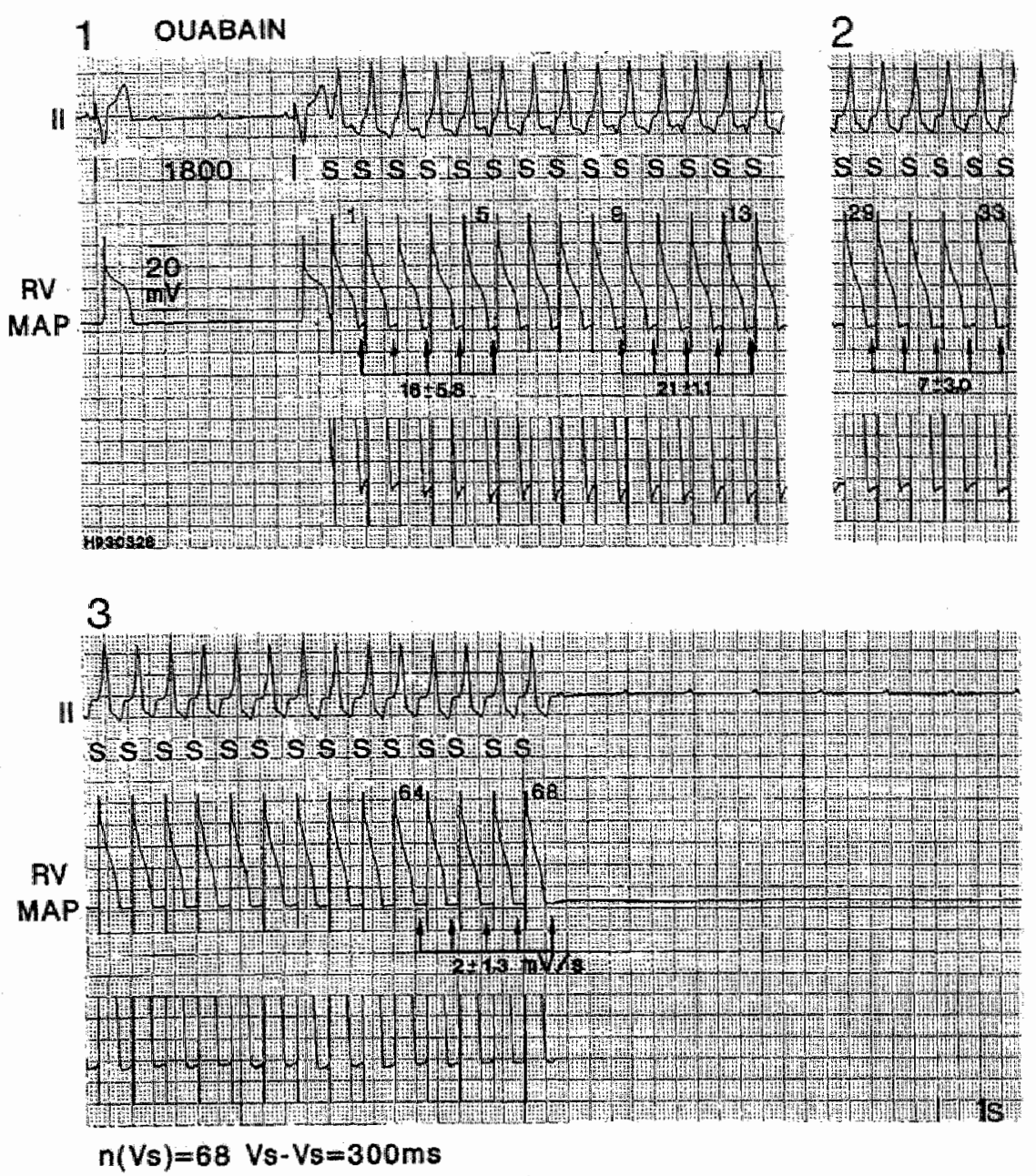

Figure 2: An individual example of the behavior of slope during a specific pacing train in the presence of ouabain is shown using ECG llead II and the monophasic action potential recording (MAP) at paper speed of $25 \mathrm{~mm} / \mathrm{second}$. In panel 1 , the idioventricular rhythm is interrupted by the start of a pacing train, with an interstimulus interval of $300 \mathrm{~ms}$ consisting of 68 stimuli (S). After 14 beats, the panel is interrupted. Continuation of pacing is shown for beats 29-33 in panel 2. Panel 3 shows the end of the pacing train (beats $64-68$ ), which is not followed by arrhythmia induction but by overdrive suppression. During the first 13 beats, slope increases from $16 \pm 5.8$ to $21 \pm 1.1 \mathrm{mV} / \mathrm{s}$, while at beats $29-33$ slope has decreased to $7 \pm 3.0 \mathrm{mV} / \mathrm{s}$ and at termination of pacing slope is flat. This biphasic behavior of the slope is especially clear in the enlarged panel, which is shown under the MAP' recording. 

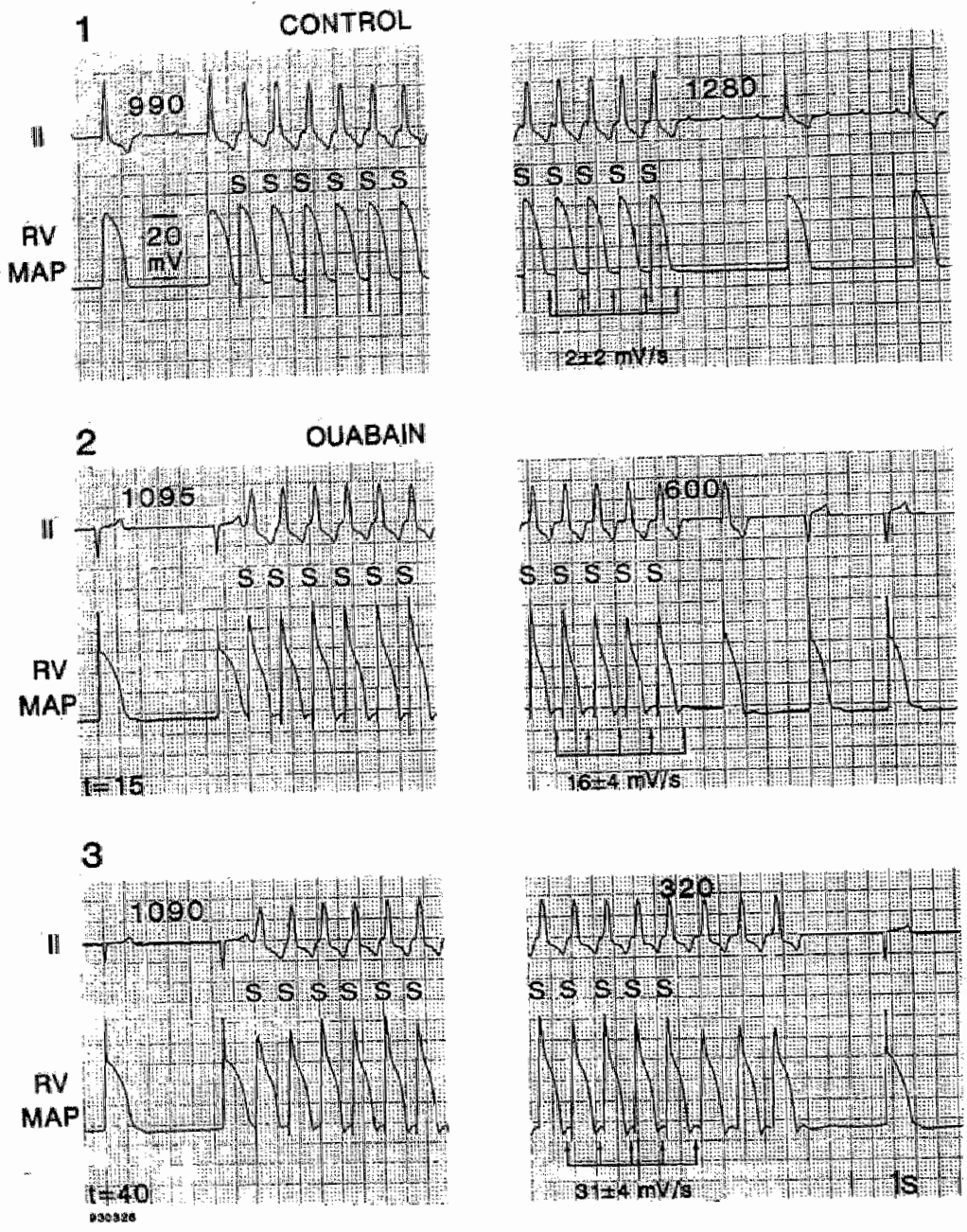

$\mathrm{n}(\mathrm{Vs})=33 \mathrm{Vs}-\mathrm{Vs}=300 \mathrm{~ms}$

Figure 3: Illustration of the time dependency of ouabain intoxication, using lead. II of the ECG and a right ventricular monophasic action potential (RV MAP) recording. Panel 1 shows the internuption of the idioventricular rate during control circumstances by the start of a pacing train ( 33 stimuli (S), interval 300 $\mathrm{ms}$ ). The panel is interrupted after 6 beats and continuation of pacing is shown at the right side of the panel. Pacing during control results in a flat slope and does not result in the induction of an ectopic beat. In panel 2, the same pacing protocol is repeated 15 minutes after ouabain, leading to an increased slope and the induction of a single ectopic beat. In panel 3, 40 minutes after ouabain, the same pacing protacol results in a steeper slope at the end of pacing and results in the induction of 3 shortly coupled ectopic beats, which are triggered. 
(duration 0 seconds), to the maximum of $25 \pm 11 \mathrm{mV} / \mathrm{s}$ (duration of 4 seconds, $\mathrm{p}<0.05$ ) at beats $9-13$. Thereafter, the slope decreased (figure 1). This dynamic behavior of the slope for a specific pacing train is illustrated in figure 2, where the slope is low at the end of pacing fails to induce an arrhythmia and is followed by overdrive suppression.

In the 6 dogs in which the pacing protocol was expanded with the $400 \mathrm{~ms}$ interstimulus interval, the measured parameters behaved similarly. The control slope was flat both at the start and the end of pacing, remaining similar for all durations ( $4 \pm 2$ and $3 \pm 3 \mathrm{mV} / \mathrm{s}$, mean \pm SD of all pacing trains). All pacing trains were followed by overdrive suppression,

Table 2: The height of the diastolic slope of the monophasic action potential measured at different durations of pacing and related to the arrhythmogenic outcome of the pacing train during ouabain

\begin{tabular}{llllc}
\hline Duration of pacing & Slope start & Slope end & VT & Number pacing train \\
\hline 04 seconds & $17 \pm 10 \mathrm{mV} / \mathrm{s}$ & $26 \pm 12 \mathrm{mV} / \mathrm{s}$ & $44 \%$ & 9 \\
10 seconds & $16 \pm 10 \mathrm{mV} / \mathrm{s}$ & $19 \pm 13 \mathrm{mV} / \mathrm{s}$ & $33 \%$ & 9 \\
20 seconds & $16 \pm 08 \mathrm{mV} / \mathrm{s}$ & $16 \pm 13 \mathrm{mV} / \mathrm{s}^{*}$ & $22 \% \%^{*}$ & 9 \\
\hline
\end{tabular}

VT $=$ ventricular tachycardia

$* p<0.05$ when compared to pacing for 4 seconds, using repeated measures ANOVA

All 3 durations of pacing were performed in 9 dogs.

which was most pronounced after pacing for 20 seconds. During ouabain, the slope at the start and the end of pacing both increased significantly $(8 \pm 5$ and $10 \pm 8 \mathrm{mV} / \mathrm{s}$, mean \pm SD of all pacing trains, $\mathrm{p}<0.05$ for both), however this increase was less pronounced when compared to $300 \mathrm{~ms}$ pacing and coincided with a less frequent induction of ventricular tachycardia. Although it did not reach statistical significance, it again was noticed that the slope was higher after pacing for 4 seconds when compared to 20 seconds (respectively $8 \pm 5 \mathrm{mV} / \mathrm{s}$ versus $12 \pm 7 \mathrm{mV} / \mathrm{s}$, figure 1 ). As during $300 \mathrm{~ms}$ pacing, this was accompanied by a higher incidence of ventricular tachycardia induction after the shortest pacing trains ( $14 \%$ compared to $0 \%$ ). 
Behavior of slope and CL prior to termination of VT

\section{Spontaneous termination}
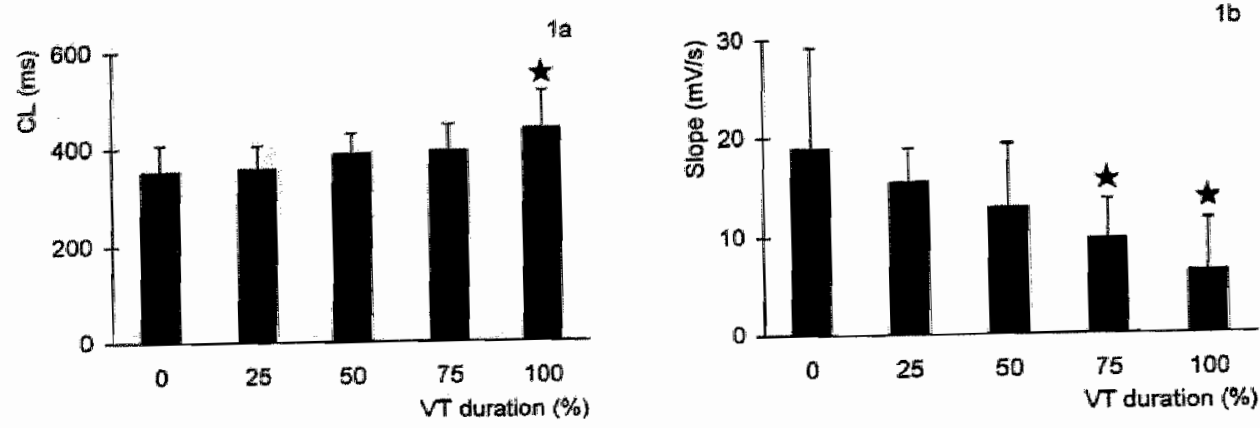

2 Lidocaine induced termination
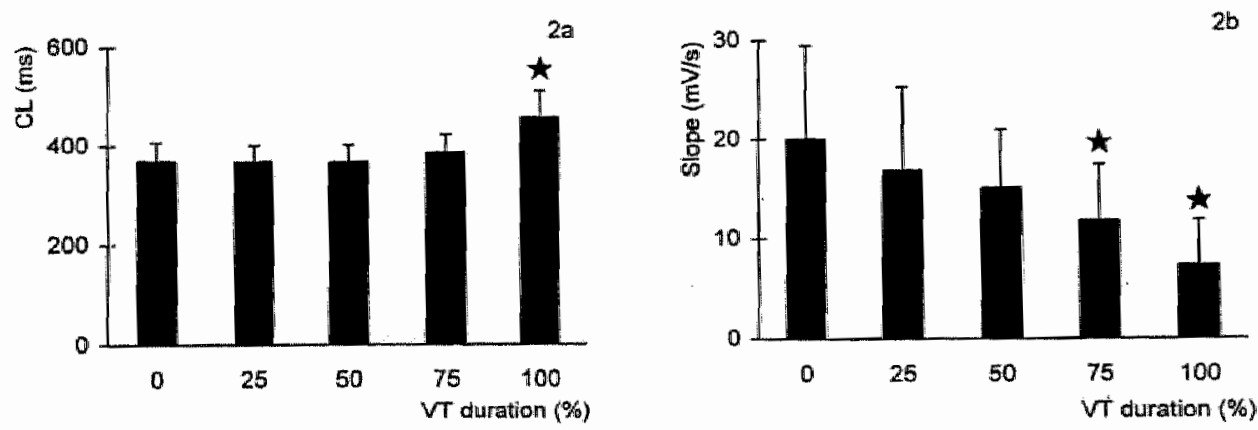

Figure 4: Histograms illustrating the behavior of ventricular tachycardia (VT) cycle length (CL) and slope prior to spontaneous termination (panel 1) and lidocaine induced termination (panel 2) of VT. On the $\mathrm{X}$-axis VT duration in percentages is indicated: $0 \%$ at the start of VT or the start of lidocaine infusion, while $100 \%$ corresponds with VT termination. There were 6 spontaneously terminating and 6 lidocaine induced VT terminations analyzed, resuiting in a mean of 6 values for each bar shown. For statistical analysis repeated measures ANOVA were used. The asterisk (*) represents significance when compared to a VT duration of $0 \%$.

Panel la shows a gradual increase in VT CL prior to spontaneous VT termination, this increase is most pronounced between a VT duration of $75 \%$ to $1.00 \%$. In panell $\mathrm{lb}$ the behavior of slope during the same period slope shows a more gradual decrease prior to termination. Panel 2 illustrates similar findings during lidocaine induced VT termination. a gradual decreasing slope (panel 2b) and a VT CL (panel 2a) that seems to react at a later time point. 

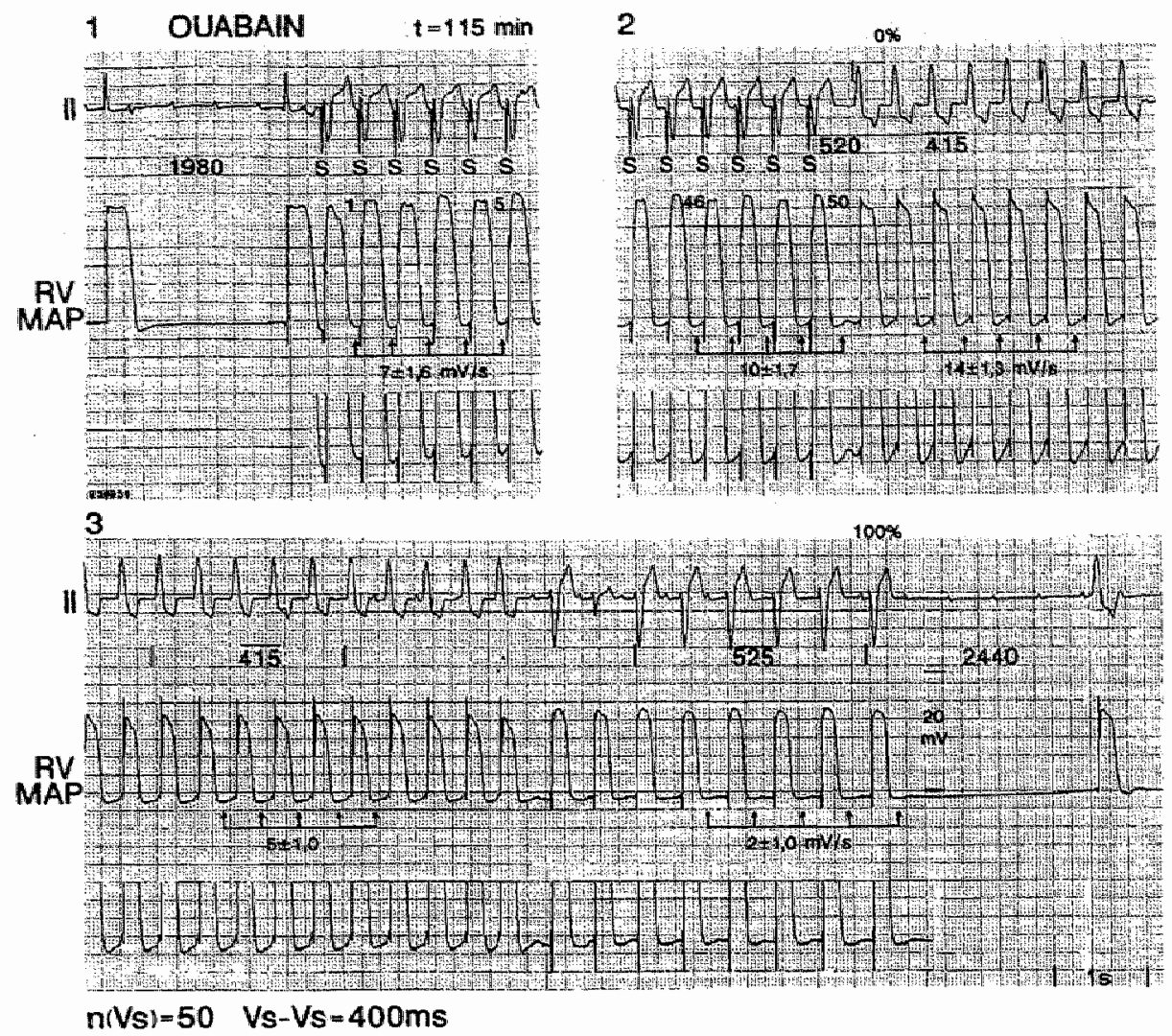

Figure 5: Spontaneous termination of ouabain induced ventricular tachycardia.

Identical composition as figure 2. Panel 1 shows the interruption of the idioventricular rate by the start of a pacing train ( 50 stimuli (S), interval $400 \mathrm{~ms}$ ) leading to induction of a ventricular tachycardia (VT) (panel 2). At the end of pacing, slope had increased from $7 \pm 1.6$ to $10 \pm 1.7 \mathrm{mV} / \mathrm{s}$. Directly after the start of VT, the slope is measured as $14 \pm 1.3 \mathrm{mV} / \mathrm{s}$ with a cycle llength (CL) of $415 \mathrm{~ms}$. In panel 3 (left), VT $\mathrm{CL}$ and slope are shown shortly before termination (panel 3, right). Decrease of the slope to $5 \pm 1.0 \mathrm{~mW} / \mathrm{s}$ results in suppression of the original VT focus, which is shortly thereafter followed by the final VT termination when slope has declined to $241.0 \mathrm{mV} / \mathrm{s}$.

\section{Group B: Behavior of the slope during ventricular tachycardia and before termination}

The time dependency of ouabain intoxication is illustrated in figure 3 for one individual dog. With progression of ouabain intoxication, the same pacing train results in steeper 
slopes and delayed afterdepolarizations with a higher amplitude, resulting in more and shorter coupled ectopic beats (figure 3 ).

The combination of ouabain and pacing resulted in the induction of ventricular tachycardias after $48 \pm 43$ minutes, who terminated spontaneously. In this period the idioventricular cycle length showed a non-significant decrease from $1482 \pm 321 \mathrm{~ms}$ to $1377 \pm 250$ $\mathrm{ms}_{\mathrm{s}}$ while the action potential duration shortened from $324 \pm 36$ to $280 \pm 28 \mathrm{~ms}(\mathrm{p}<0.05)$. After $71 \pm 57$ minutes of ouabain and pacing, ventricular tachycardias with a duration of more than 5 minutes were induced. These ventricular tachycardias were terminated with lidocaine. In this group, the idioventricular cycle length had decreased from $1514 \pm 410$ $\mathrm{ms}$ during control to $1253 \pm 446 \mathrm{~ms}(\mathrm{p}<0.05)$, while action potential duration shortened from $333 \pm 33$ to $275 \pm 40 \mathrm{~ms}(\mathrm{p}<0.05)$.

\section{Spontaneous ventricular tachycardia termination}

Delayed afterdepolarizations were seen in the monophasic action potential recordings at the moment of ventricular tachycardia induction and during all ventricular tachycardias. The 6 spontaneously terminating ventricular tachycardias had a duration of $176 \pm 227$ seconds. Ventricular tachycardia cycle length was $353 \pm 54 \mathrm{~ms}$ at initiation of ventricular tachycardia, which gradually increased towards $434 \pm 78 \mathrm{~ms}$ at termination $(\mathrm{p}<0.05$, figure 4 panel 1a). The diastolic slope decreased in this period from $19 \pm 10 \mathrm{mV} / \mathrm{s}$ at the start of ventricular tachycardia to $6 \pm 5 \mathrm{mV} / \mathrm{s}$ at termination ( $<<0.05$, figure 4 panel $1 \mathrm{~b}$ ). An example of the behavior of a spontaneously terminating ventricular tachycardia is given in figure 5 .

\section{Lidocaine induced ventricular tachycardia termination}

In 6 experiments, ouabain induced sustained ventricular tachycardia (mean duration $12 \pm 6$ minutes, figure 6) was terminated with lidocaine. At ventricular tachycardia initiation, cycle length was $415 \pm 91 \mathrm{~ms}$ and slope was $17 \pm 8 \mathrm{mV} / \mathrm{s}$. Again delayed afterdepolarizations were seen in the monophasic action potential recordings during all ventricular tachycardias. From ventricular tachycardia initiation to start of lidocaine, the ventricular tachycardia cycle length significantly decreased to $370 \pm 37 \mathrm{~ms}$ and slope increased to $20 \pm 9 \mathrm{mV} / \mathrm{s}$ (NS). Prior to ventricular tachycardia termination, ventricular tachycardia rate slowed to a cycle length of $454 \pm 54 \mathrm{~ms}(\mathrm{p}<0.05)$ and slope decreased to $7 \pm 4 \mathrm{mV} / \mathrm{s}$ ( $p<0.05$, figure 4 panel 2). Lidocaine terminated all ventricular tachycardias, at $95 \pm 39$ seconds after the start of lidocaine infusion. 


\section{Discussion}

The results of this study indicate that the diastolic slope seen in the monophasic action potential recordings varies (dynamic behavior) during pacing, and can be related to the arrhythmogenic outcome post-pacing. Furthermore, during ventricular tachycardia changes in the slope can be used to identify delayed afterdepolarization-dependent ventricular tachycardia and can be related to (drug induced) ventricular tachycardia termination.

\section{Monophasic action potential recordings}

With monophasic action potential recordings, basic electrophysiological phenomena can be registered in the intact beating heart. ${ }^{17,18}$ Although monophasic action potential recordings are not identical to transmembrane action potentials, they can be used for the detection of afterdepolarizations in vivo. ${ }^{17,18}$ However, general application of this technique for this purpose still encounters some practical problems, including monophasic action potential stability and registration of artefacts. ${ }^{18}$ Therefore, expertise, high quality equipment, and the application of (pharmacological) interventions are necessary to identify afterdepolarizations with a high degree of certainty. ${ }^{12}$ With this consideration, we have recently described the use of the monophasic action potential to identify both early and delayed afterdepolarizations in the intact dog heart. ${ }^{12,19}$ Similarly in this study, we have controlled monophasic action potential stability (table 1). Based on the interventions performed in relation to arrhythmogenic outcome, we do not consider the changes in the diastolic slope to be artificial.

\section{Model}

The animal model of ouabain induced ventricular tachycardias used in this study, has previously been studied in conscious and anesthetized dogs. ${ }^{12,16,20-22}$ The induction of these arrhythmias is facilitated by combining ouabain administration with pacing. ${ }^{16,22,23}$ At the concentration of ouabain used, it takes approximately 70 minutes before sustained ventricular tachycardias (duration longer than 5 minutes) can be induced. This progressive behavior of intoxication leads to a steeper diastolic slope during pacing, an increased delayed afterdepolarization amplitude, an increasing incidence of triggered ectopic beats with a shorter coupling interval (figure 3 ) and the initiation of ventricular tachycardia. ${ }^{16,20,21}$ It has been suggested that partial inhibition of the $\mathrm{Na}^{+} / \mathrm{K}^{+}$pump by ouabain causes an increase in $\left[\mathrm{Na}^{+}\right]$i concentration, that through the $\mathrm{Na}^{+} / \mathrm{Ca}^{2+}$ exchanger, will result in an increased $\left[\mathrm{Ca}^{2+}\right] \mathrm{i} .{ }^{24-27}$ Additionally, pacing will result in a sodium (and 
to a lesser extent calcium) accumulation that further increases this $\left[\mathrm{Ca}^{2+}\right] \mathrm{i}$ loading, which causes the sarcoplasmic reticulum to oscillatory release of $\mathrm{Ca}^{2+}$ in the cytosol, thereby eliciting an transient inward current $\left(\mathrm{I}_{\mathrm{ti}}\right)$ which is responsible for the delayed afterdepolarizations. ${ }^{24-27}$ Proposed mechanisms underlying the $\mathrm{Iti}_{\mathrm{ti}}$ are the $\mathrm{Na}^{+} / \mathrm{Ca}^{2+}$ exchanger or a calcium activated non specific channel. ${ }^{24-27}$

\section{Behavior of slope during pacing}

In this and a previous study ${ }^{12}$, the behavior of the slope at the end of the pacing train was related to the outcome: arrhythmia induction or no arrhythmia induction. For the first time, we now describe that under ouabain circumstances not sufficient to induce ventricular tachycardia (subtoxic ouabain), the height of the diastolic slope can vary
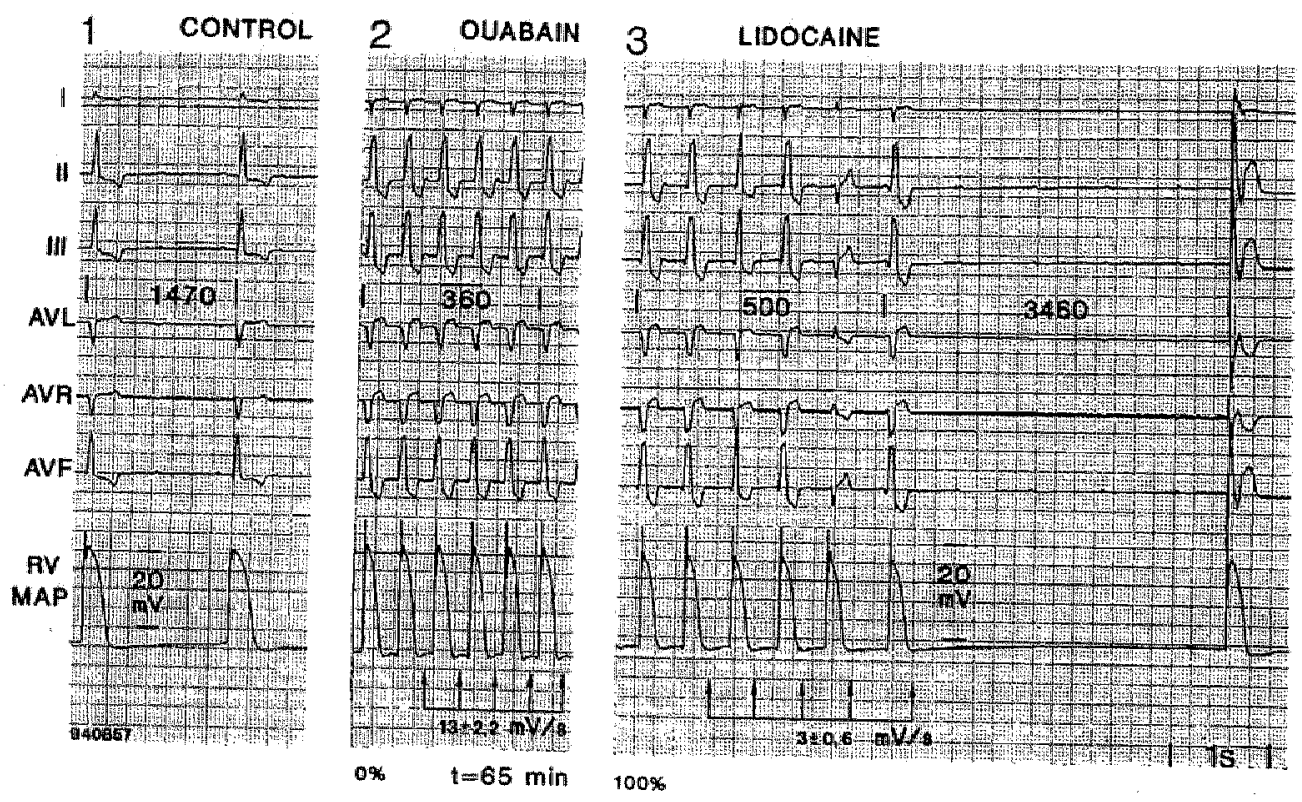

Figure 6: Lidocaine induced termination of ouabain induced ventricular tachycardia (VT). In each panel 6 ECG leads and a monophasic action potential (MAP) recording is shown at a paper speed of 25 $\mathrm{mm} / \mathrm{second}$. Panel 1 shows the control situation in which the idioventricular cycle length is $1470 \mathrm{~ms}$. The MAP shows no DADs and the slope is flat. In panel 2, a VT with a cycle length (CL) of $360 \mathrm{~ms}$ and a slope of $13 \pm 2.2 \mathrm{mV} / \mathrm{s}$ is seen, just before the lidocaine infusion was started. Lidocaine administration resulted in VT termination, as shown in panel 3. Note that the DADs and the slope, that were clearly visible in panel 2 , are completely suppressed. The first beat after termination of VT has been paced, because of temporary cessation of the rhythm. 
during ventricular pacing. The slope shows a biphasic behavior: an initial increase is followed by a decrease depending on the duration of pacing.

Under control circumstances, this behavior was not seen: the slope was and remained flat during pacing which coincided with the inability to induce arrhythmias. After 15-30 minutes of ouabain, the height of the diastolic slope during pacing varied being most pronounced after 4 seconds of pacing (when pacing with 300 and $400 \mathrm{~ms}$ interstimulus intervals). When pacing was stopped at this moment, the chances that a ventricular arrhythmia could be induced were the highest. Continuation of pacing resulted in suppression of the slope to baseline values and the inability to induce arrhythmias. This pattern of behavior was present during both interstimulus intervals used, although the maximal slope was less high during pacing with $400 \mathrm{~ms}$. This corresponds with the less frequent induction of ventricular tachycardia, which is in agreement with the tachycardia dependency of delayed afterdepolarizations. $1,2,10,11,26,28$

When we speculate about the biphasic behavior of the slope and the ability versus the inability to induce triggered beats, we consider a role of the $\mathrm{Na}^{+} / \mathrm{K}^{+}$ATP-ase as explanation plausible. Overdrive pacing of the idioventricular rhythm normally results in overdrive suppression by activation of the $\mathrm{Na}^{+} / \mathrm{K}^{+}$ATP-ase. ${ }^{29}$ The amount of overdrive suppression is dependent on the duration and rate of stimulation. ${ }^{29}$ Besides overdrive suppression, we also noted the initiation of ectopic beats under control conditions when pacing was performed for a short time (10 stimuli) with a short interstimulus interval $(200 \mathrm{~ms}),{ }^{20}$ in conscious dogs with chronic complete atrioventricular-block. Since the $\mathrm{Na}^{+} / \mathrm{K}^{+}$ATP-ase is activated with a time delay regarding onset of pacing, ${ }^{29}$ in the beginning of pacing the intracellular sodium will quickly rise. This non compensated increase in intracellular sodium can lead to an increase in intracellular calcium with the occurrence of delayed afterdepolarizations, as is suggested using the slope. When the activity of the $\mathrm{Na}^{+} / \mathrm{K}^{+}$ATP-ase catches up, the slope will start to decrease. Thus depending on its duration and/or rate, pacing can either result in the induction of delayed afterdepolarization-dependent beats or overdrive suppression. Administration of ouabain, an inhibitor of the $\mathrm{Na}^{+} / \mathrm{K}^{+}$ATP-ase, will increase the ability to induce arrhythmias and decrease the number of trains that are followed by overdrive suppression. The comparison of the frequency dependency is also in accordance. Using the same durations of pacing, the slope will increase more during $300 \mathrm{~ms}$ pacing, most probably related to a larger sodium influx in time. Similar data have been obtained but not published as such in our previous publication. ${ }^{12}$ Both during control and lidocaine circumstances, the slope was flat at the end of the pacing train, independent of the 
duration of pacing. This is in contrast to the ouabain circumstances, where the biphasic behavior was observed when the different durations of pacing were compared. Therefore, the increase in slope after ouabain indicates the presence of delayed afterdepolarizations $^{12}$ and corresponds with delayed afterdepolarization behavior described in isolated tissue preparations. ${ }^{1,2,30}$ In this setting, the reduction of slope to baseline values can be seen as an adjustment to recover from the initially induced $\mathrm{Ca}^{2+}$ overload and related delayed afterdepolarizations. Prolonged pacing will provide the time to activate the counteracting forces by stimulating the $\mathrm{Na}^{+} / \mathrm{K}^{+}$-ase. In line with this hypothesis is the observation that activation of the $\mathrm{Na}^{+} / \mathrm{K}^{+}$-ase pump sometimes even seems able to suppress sustained triggered activity in isolated tissue preparations. ${ }^{31}$

\section{Behavior of ventricular tachycardia prior to termination}

The second objective was to study the influence of delayed afterdepolarizations in the perpetuation of ventricular tachycardia, by quantifying the behavior of ventricular tachycardia cycle length and the diastolic slope of the monophasic action potential. The amplitude or slope of the delayed afterdepolarizations might be represented in the ventricular tachycardia cycle length, because diminishment of these parameters will lead to slowing of the ventricular tachycardia rate and termination. ${ }^{1-9}$

Our data show a high diastolic slope of the monophasic action potential during ventricular tachycardia, which decreases towards ventricular tachycardia termination. This corresponds with observations made in isolated tissue preparations, where, during sustained triggered activity, the delayed afterdepolarization that triggers an action potential is only visible as a steep diastolic slope. Towards termination of the spontaneous activity, delayed afterdepolarization amplitude and the diastolic slope decline. ${ }^{3,4}$

Lidocaine is known to suppress delayed afterdepolarizations and related arrhythmias in isolated tissue $\mathrm{e}^{32}$ and in the intact heart. ${ }^{33}$ Although it is not a delayed afterdepolarization specific drug, it is rapidly effective in suppressing delayed afterdepolarization amplitude $^{32}$ and is well tolerated by dogs. ${ }^{33}$ During lidocaine, the slope showed a gradual decrease from the start of lidocaine infusion to ventricular tachycardia termination. Similarly, ventricular tachycardia cycle length increased towards termination. However, there was a discrepancy in time dependence: the major increase in cycle length occurred later than the major decrease in slope, both during spontaneous and lidocaine induced ventricular tachycardia termination. Possibly this can be explained by a late switch from the first to a second delayed afterdepolarization ${ }^{26}$ by which cycle length would increase, while the slope of the first delayed afterdepolarization would remain similar. However, 
we did never observed two delayed afterdepolarizations in the monophasic action potential recordings in these experiments.

\section{Limitations}

Slope was measured in a randomly placed monophasic action potential recording, without considering the site of delayed afterdepolarization origin in relation to the site of recording. A stable monophasic action potential signal with a certain amplitude was considered to be of greater importance than a search for specific sites at which delayed afterdepolarizations occur. ${ }^{34}$ We used a globally intoxicated dog heart and do not know to what extent our registrations of delayed afterdepolarizations and slope are a true manifestation of delayed afterdepolarizations at the site of origin. Other experiments are necessary to evaluate application of our observations during (more localized) pathological circumstances of intracellular calcium overload and during ventricular tachycardias with unknown underlying mechanism.

In summary, when we assume that the diastolic slope of monophasic action potential recordings represents delayed afterdepolarizations, we conclude that 1) both induction and perpetuation of ouabain induced ventricular tachycardia are delayed afterdepolarization-dependent, 2) the height of the slope during pacing seems to be related to the arrhythmogenic outcome, 3) this slope varies depending on the duration of pacing, and 4) the slope during ventricular tachycardia might be useful as a diagnostic tool to study the effect of interventions in delayed afterdepolarization-dependent arrhythmias.

\section{Acknowledgments}

The authors would like to thank the Bakken Research Center, Maastricht, The Netherlands for supplying the electrodes and the Netherlands. Organization for Scientific Research for providing a travel grant. This study was supported by a grant from the Wynand N. Pon Foundation, Leusden, The Netherlands.

\section{References}

1. Ferrier $\mathrm{GR}$, Saunders JH, Mendez $\mathrm{C}$. A cellular mechanism for the generation of ventricular arrhythmias by acetylstrophanthidin. Circ Res 1973; 32: 600-609.

2. Rosen MR, Gelband H, Merker C, Hoffman BF. Mechanisms of digitalis toxicity. Effects of ouabain on phase four of canine Purkinje fiber transmembrane potentials. Circulation 1973; 47 : 681-689.

3. Cranefield PF, Aronson R. Cardiac arrhythmias: The role of triggered activity and other mechanisms. New York.: Nt Kisco, New York, Futura publishing Co., 1988. 
4. Le Marec H, Spinelli $W$, Rosen MR. The effects of doxorubicin on ventricular tachycardia. Circulation 1986: 74:881-889.

5. Wit AL, Cranefield PF. Triggered and automatic activity in the canine coronary sinus. Circ Res $1977 ; 41: 434-445$.

6. Ferrier GR, Moffat MP, Lukas A. Possible mechanisms of ventricular arthythmias elicited by ischemia followed by reperfusion. Studies on isolated canine wentricular tissues. Circ Res 1985; 56 : 184-194.

7. Hiraoka M, Okamoto $\mathrm{Y}$, Sano $\mathrm{T}$. Oscillatony afterpotentials in dog ventricular muscle fibers. Circ Res 1981; 48: 510-518.

8. Le Marec H, Dangman KH, Danilo P, Rosen MR. An evaluation of automaticity and triggered activity in the canine heart one to four days after myocardial infarction. Circulation 1985; 71: 12241236.

9. Aronson R.S. Afterpotentials and triggered activity in hypertrophied myocardium from rats with renal hypertension. Circ Res 1981; 48: 720-727.

10. Priori SG, Mantica M, Schwartz PJ. Delayed afterdepolarizations elicited in vivo by left stellate ganglion stimulation. Circulation 1988; 78: 178-185.

11. Furukawa $T$, Kimura $S$, Castellanos A, Bassett AL, Myerburg RJ. In wivo induction of "focal" triggered ventricular arrhythmias and responses to overdrive pacing in the canine heart. Circulation 1990; 82: 5449-559.

12. De Groot SHM, Vos MA, Gorgels APM, Leunissen IDM, van der Steld BJ, Wellens HJJ. Combining monophasic action potential recordings with pacing to demonstrate delayed afterdepolarizations and triggered arthythmiss in the intact heart. Circulation 1995; 92: 2697-2704.

13. Xu J, Hurt CM, Pelleg A. Digoxin-induced ventricular arthythmias in the guinea pig heart in vivo: Evidence for a role of endogenous catecholamines in the genesis of delayed afterdepolarizations and triggered activity. Heart and Vessels 1995; 10:119-127.

14. Steiner C, Kovalik AT. A simple technique for production of chronic complete heart block in dogs. J Appl Physial 1968; 25: 631-632.

15. Franz MR, Chin MC, Sharkey HR, Griffin JC, Scheinman MM. A new single catheter technique for simultaneous measurement of action potential duration and refractory period in vivo. $J \mathrm{Am} \mathrm{Coll}$ Candiol 1990; 16:878-886.

16. Vos MA, Gorgels AP, Leunissen JD, van Deursen RT, Wellens HJJ. Significance of the number of stimuli to initiate ouabain-induced arrhythmias in the intact heart. Circ Res 1991; 68: 38-44.

17. Zipes DP. Monophasic action potentials in the diagnosis of triggered arthythmias. Prog Cardiovasc Dis 1991; $33: 385-396$.

18. Franz MR. Bridging the gap between basic and clinical electrophysiology: What can be learned from monophasic action potential recordings. J Cardiovasc Electrophysiol 1994; 5: 699-710.

19. Vos MA, Verduyn SC, Gorgels AP, Lipcsei GC, Wellens HJ. Reproducible induction of early afterdepolarizations and torsade de pointes arrhythmias by d-sotalol and pacing in dogs with chronic atrioventricular block. Circulation 1995; 91 : 864-872.

20. Vos MA, Gorgels AP, de Wit B, Drenth JP, van Deursen RT, Leunissen JD, Wellens HJ Premature escape beats. A model for triggered activity in the intact heart? Circulation 1990; 82: 213-224.

21. Hagemeijer $F$, Lown B. Effect of heart rate on electrically induced repetitive ventricular responses in the digialized dog. Circ Res $1970 ; 27: 333-344$.

22. Gorgels AP, De Wit B, Beekman HD, Dassen WR, Wellens HJ. Triggered activity induced by pacing during digitalis intoxication observations during programmed electrical stimulation in the conscious dog with chronic complete atrioventricular block. P.ACE 1987; 10: 1309-1321. 
23. Wittenberg SM, Streuli F, Klocke FJ. Acceleration of ventricular pacemakers by transient inereases in heart rate in dogs during ouabain administration. Circ Res 1970; 26: 705-716.

24. Berlin JR, CannelI MB, Lederer WJ. Cellular origins of the transient inward current in cardiac myocytes. Role of fluctuations and waves of elevated intracellular calcium. Circ Res 1989;65: 115-126.

25. Fedida $\mathrm{D}$, Noble $\mathrm{D}$, Rankin $\mathrm{AC}$, Spindler $\mathrm{AJ}$. The arrhythmogenic transient inward current Iti and related contraction in isolated guinea-pig ventricular myocytes. I Physiol (Lond) 1987; 392: 523542.

26. Rosen MR. Delayed afterdepolarizations induced by digitalis. In: Rosen MR, Janse MJ, Wit AL, Eds. Cardiac electrophysioloy: a textbook. New York: Mount Kisco, Futura, 1990: 271-281.

27. Smith TW. Digitalis. Mechanisms of action and clinical use. N Engl J Med 1988; 318; 358-365.

28. Rosen MR, Fisch C, Hoffman BF, Danilo P, Lovelace DE, Knoebel SB. Can accelerated atrioventricular junctional escape rhythms be explained by delayed afterdepolarizations? Am J Cardiol $1980 ; 45: 1272-1284$.

29. Vassalle $\mathrm{M}$, Cummins $\mathrm{M}$, Castro $\mathrm{C}$, Stuckey JH. The relationship between overdrive suppression and overdrive excitation in ventricular pacemakers in dogs. Circ Res 1976; 38: 367-374.

30. Johnson N, Danilo P, Wit AL, Rosen MR. Characteristics of initiation and termination of catecholamine-induced triggered activity in atrial fibers of the coronary simus. Circulation 1986; 74: 1168 1179 .

31. Wit AL, Cranefield PF, Gadsby DG. Electrogenic sodium extrusion can stop triggered activity in the canine coronary sinus. Circ Res 1981; 49: 1029-1042.

32. Rosen MR, Danilo P. Effects of tetrodotoxin, lidocaine, verapamil, and AHR-2666 on Ouabain-induced delayed afterdepolarizations in canine Purkinje fibers. Circ Res 1980; 46: 117-124.

33. Vos MA, Gorgels APM, Leunissen HDM, Korterink W, Wellens HJJ. Discriminative power of drugs to identify specific arrhythmogenic mechanisms in vivo. Cardiovasc Drug Ther 1991; 5:402. (abstract)

34. Gorgels AP, De WitB, Beekman HD, Dassen WR, Wellens HJ. Effects of different modes of stimulation on the morphology of the first QRS complex following pacing during digitalis-induced ventricular tachycardia observations in the conscious dog with chronic complete atrioventricular block. PACE 1986; 9: 842-859. 



\title{
Chapter 4
}

\section{Dualistic effects of ryanodine on ouabain induced delayed afterdepolarization-dependent ventricular tachycardia}

\author{
Marieke de Groot \\ Marc Vos \\ Jet Leunissen \\ Marieke Schoenmakers \\ Theo van der Nagel \\ Hein Wellens
}

Abstract presented at the North American Society of Pacing and Electrophysiology, 1996 (PACE 1996; 19: 604)

Submitted for publication 


\section{Abstract}

Introduction: Ryanodine is a specific blocker of the sarcoplasmic reticulum $\mathrm{Ca}^{2+}$ release channel and suppresses delayed afterdepolarization-dependent ouabain induced ventricular tachycardia. In conscious dogs with complete atrioventricular-block, ryanodine $(10 \mu \mathrm{g} / \mathrm{kg})$ initially accelerated ouabain induced ventricular tachycardia, before slowing and termination occurred. To elucidate the cause of this acceleration, ryanodine was given to anesthetized atrioventricular-block dogs during 1) ouabain induced ventricular tachycardia $(\mathrm{n}=7)$ and 2) idioventricular rhythm $(\mathrm{n}=6)$.

Methods: To investigate the time dependent relation between cycle length, delayed afterdepolarizations (recorded with monophasic action potentials in both ventricles) and left ventricular pressure, we determined the diastolic slope $(\mathrm{dV} / \mathrm{dt})$ of the delayed afterdepolarization, action potential duration, and $+\mathrm{LV} \mathrm{dP} / \mathrm{dt}$. Reflex tachycardia was excluded as cause of rate acceleration by giving ryanodine after autonomic blockade ( 1 $\mathrm{mg}$ atropine and $0.3 \mathrm{mg} / \mathrm{kg}$ propranolol $)$ and by giving verapamil $(0.5 \mathrm{mg} / \mathrm{kg})$ to terminate ventricular tachycardia.

Results: Ryanodine terminated ventricular tachycardia in $7 / 7$ dogs after $12 \pm 2$ minutes. This was preceded by a decrease of ouabain induced ventricular tachycardia cycle length $368 \pm 31$ to $336 \pm 32 \mathrm{~ms}(\mathrm{p}<0.05)$ after $7 \pm 2$ minutes. In this period, action potential duration remained similar in both ventricles. Ventricular tachycardia acceleration was preceded by an increase of the $+\mathrm{LV} \mathrm{dP} / \mathrm{dt}$ and persisted during autonomic blockade. Before ouabain induced ventricular tachycardia termination, cycle length increased to $421 \pm 32 \mathrm{~ms}$ $(\mathrm{p}<0.05)$. This was accompanied by a decrease 1$)$ in slope of both monophasic action potentials and 2) in $+\mathrm{LV} \mathrm{dP/dt}$. Verapamil did not cause a rate acceleration. Without autonomic blockade, ryanodine decreased the $+\mathrm{LV} \mathrm{dP} / \mathrm{dt}$ and accelerated idioventricular rhythm (both $\mathrm{p}<0.05$ ). With autonomic blockade, the rate acceleration was prevented. Independent of autonomic blockade, ryanodine exerted an initial positive inotropic effect on $+\mathrm{LV} \mathrm{dP} / \mathrm{dt}$.

Conclusions: Ryanodine terminates delayed afterdepolarization-dependent ouabain induced ventricular tachycardia. The anti-arrhythmic effect is preceded by an agonistic effect, causing ventricular tachycardia acceleration. This effect on the ouabain induced ventricular tachycardia rate seems to be related to an effect of ryanodine on calcium release, which is also expressed by the transient increase in left ventricular contractile function. 


\section{Introduction}

The arrhythmogenic mechanism of triggered activity based on delayed afterdepolarizations has been described during a variety of circumstances, including digitalis intoxication, catecholamine excess, ischemia, reperfusion, and ventricular hypertrophy. ${ }^{1}$ The common denominator for the occurrence of delayed afterdepolarizations under these circumstances is a (too) high intracellular calcium concentration. During this condition, it is more likely for the sarcoplasmic reticulum to spontaneously release calcium, resulting in the occurrence of 1) after contractions and 2) afterdepolarizations, which when there amplitude reaches threshold will lead to ectopic activity and possibly ventricular tachycardias. ${ }^{2}$

Different antiarrhythmic drugs may prevent or suppress digitalis-induced arrhythmias. ${ }^{3-}$ ${ }^{10}$ The anti-arrhythmic action of these drugs is often accompanied by a negative inotropic effect, because they reduce the increased myocardial calcium concentration. One of these anti-arrhythmic drugs is ryanodine, which blocks the $\mathrm{Ca}^{2+}$ release channel of the sarcoplasmic reticulum. ${ }^{11,12}$ Ryanodine has been described to suppress delayed afterdepolarization-dependent tachyarrhythmias both in vivo ${ }^{7,8}$ and in vitro. ${ }^{9}$ In preliminary experiments, we studied the effect of ryanodine on ouabain induced ventricular tachycardia in conscious dogs with chronic complete atrioventricular-block and found that ryanodine initially caused an acceleration in rate, followed by slowing and termination of ventricular tachycardia. Although a pro-arrhythmic response with low plasma concentration ryanodine has been described previously for early afterdepolarization-related arrhythmias, ${ }^{13,14}$ this acceleration of delayed afterdepolarization-dependent ventricular tachycardia rate seems to be unique for ryanodine and was never observed in our atrioventricular-block, model with other anti-arrhythmic drugs where termination of the delayed afterdepolarization-dependent ventricular tachycardia is preceded by a slowing in rate. ${ }^{3-6,15}$ It was the aim of the present study to further investigate the effect of ryanodine on ouabain induced ventricular tachycardia with special emphasis on the rate acceleration. In order to elucidlate the underlying mechanism, we studied the time-dependent relation between rate, delayed afterdepolarization-slope and left ventricular pressure.

\section{Methods}

All experiments were performed in accordance with the "Guiding principles in the care and use of animals" as approved by the American Physiological Society and under the 


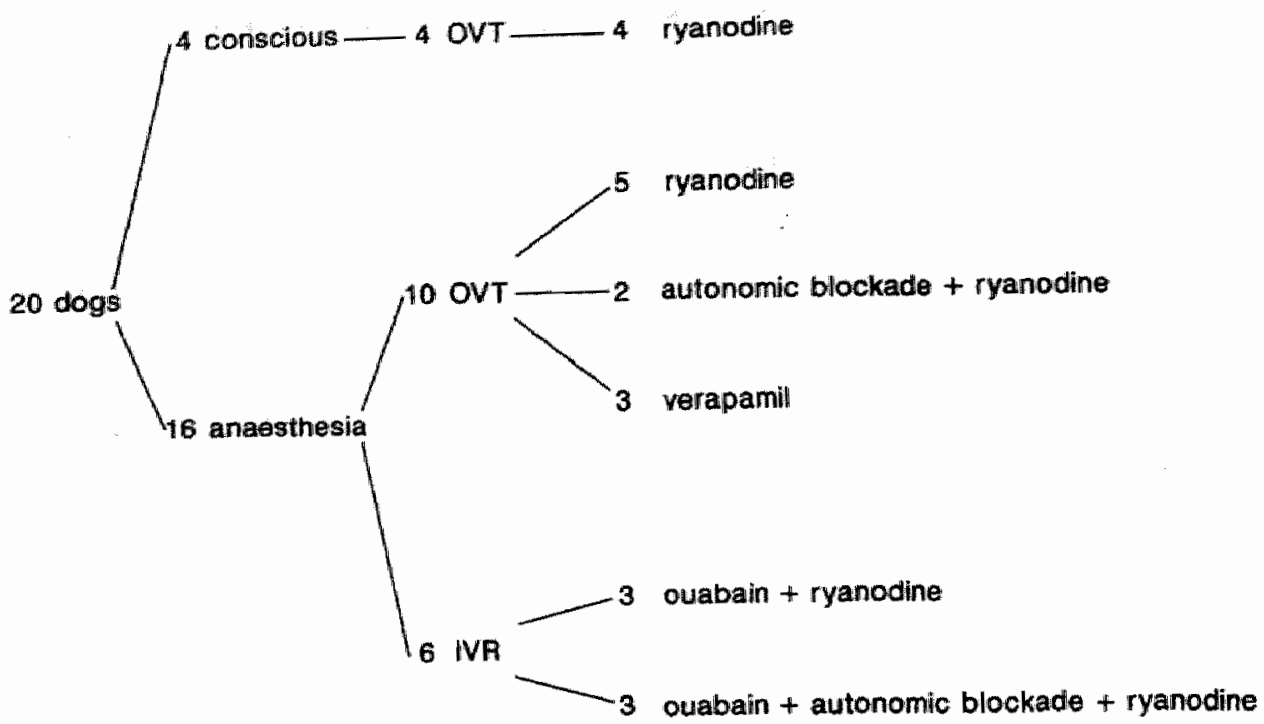

Figure 1: Flow chart of the protocol used in the 20 dogs studied. All dogs that were studied during ouabain induced ventricular tachycardia (OVT) are in protocol A, while all dogs studied during idioventricular rhythm (TVR) are in protocol $\mathrm{B}$.

Dutch Law on Animal Experimentation and the European Directive for the Protection of Vertebrate Animals used for Experimental Purposes. Additionally, all experiments were approved by the Animal Investigation Committee of the Maastricht University.

\section{Dog model of complete atrioventricular-block}

The studies were performed in 4 conscious and 16 anesthetized adult mongrel dogs (mean body weight of $27 \pm 4 \mathrm{~kg}$ ) of either sex (figure 1). Complete atrioventricular-block was made to exclude the interference of conducted sinus beats with ventricular arrhythmias. ${ }^{16,17}$ During the atrioventricular-block operation an epicardial screw in electrode was placed in the apex of the left ventricle. The experiments were performed after $6 \pm 2$ weeks after the operation, with a minimal delay of 2 weeks. During all experiments, values of RR intervals were continuously monitored using a computerized $Q R S$ detecting system. Six surface ECG leads were registered and stored on the hard disc of the 
computer. Monophasic action potential-signals and left ventricular pressure signals were simultaneously registered and stored. Drugs were administered through a cannula in a cephalic vein.

\section{Anesthesia}

In experiments using anesthetized animals, premedication $(0.2 \mathrm{ml} / \mathrm{kg})$ was given intramuscularly with a drug mixture containing $10 \mathrm{mg}$ oxycodan $\mathrm{HCl}, 1 \mathrm{mg}$ acepromazine and $0.5 \mathrm{mg}$ atropine sulfate per $\mathrm{ml}$. Experimental procedures were performed under sterile conditions. An antibiotic (1000 mg ampicillin) was given preoperatively (IV) and post-operatively (IM). Anesthesia was induced by sodium pentobarbital ( $20 \mathrm{mg} / \mathrm{kg} \mathrm{IV}$ ). The dogs were artificially ventilated (Pulmomat respirator, Dräger), through a cuffed endotracheal tube with a mixture of oxygen, nitrous oxide and halothane (vapor concentration $0.5 \%$ ). Ventilation was adjusted on the basis of continuous monitoring of the carbon dioxide concentration of the expired air. A thermal mattress was used to maintain body temperature around $37^{\circ} \mathrm{C}$ during the experiments.

\section{Induction of ventricular tachycardia (protocol A)}

Sustained ventricular tachycardia was induced by combining ventricular pacing with ouabain administration in $14 \mathrm{dogs} ; 4$ conscious dogs, lying quietly on the floor and 10 anesthetized dogs (figure 1).

Using a programmable stimulator having a synchronizing circuit, the heart was stimulated from the left ventricular epicardial electrode (twice diastolic threshold, pulse width $2 \mathrm{~ms}$ ). Pacing was performed using 3 different stimulation intervals $(200,300$ and 400 $\mathrm{ms}$ in conscious dogs and 250,300 and $400 \mathrm{~ms}$ in anesthetized dogs) and 3 different durations of pacing $(4,10$ and 20 seconds). In between pacing, enough time was allowed to regain the prepacing idioventricular rhythm. Pacing was performed during control, resumed 15 minutes after the start of ouabain administration and repeated every 15 minutes until ventricular tachycardia induction. Ouabain was administered as a bolus in relation to body weight ${ }^{15}$ ( 1 minute bolus: $46 \pm 3 \mu \mathrm{g} / \mathrm{kg}$ in conscious dogs and $48 \pm 6 \mu \mathrm{g} / \mathrm{kg}$ during anesthesia), followed after 10 minutes by a continuous infusion (range 0.072 to $0.081 \mu \mathrm{g} / \mathrm{kg} /$ minute in conscious dogs and 0.072 to $0.090 \mu \mathrm{g} / \mathrm{kg} /$ minute in anesthetized dogs).

Ventricular tachycardia was defined as a ventricular rhythm with a cycle length shorter than $600 \mathrm{~ms}$. Before starting with administration of any (anti-arrhythmic) drug, the ventricular tachycardia was observed at least 20 minutes. During the first 10 minutes 
after ventricular tachycardia induction, the rate shows a tendency to accelerate. ${ }^{18}$ By following the rate for at least 20 minutes, we ensured a stable cycle length for minimally 10 minutes before drugs were administered. Polymorphic or hemodynamically badly tolerated ventricular tachycardias were not used to study the effect of ryanodine or verapamil.

\section{Administration of ryanodine and verapamil}

Ryanodine $(10 \mu \mathrm{g} / \mathrm{kg}$ in 10 minutes) was given to terminate ouabain induced ventricular tachycardia in 4 conscious dogs. To further examine the time-dependent behavior of the rate of ventricular tachycardia in relation to delayed afterdepolarization-slope and left ventricular pressure, 7 experiments in anesthetized dogs were also performed (protocol A figure 1). Ryanodine has been described to exert a pronounced negative inotropic effect $^{7}$ which might cause a rate acceleration by a reflex mediated sympathetic mechanism. To prevent such mechanism from occurrence, autonomic blockade ${ }^{19}$ was induced 10-15 minutes before ryanodine in a number of experiments (figure 1). For this purpose, $1 \mathrm{mg}$ per 1 minute of atropine followed by $0.3 \mathrm{mg} / \mathrm{kg}$ per 3 minutes of propranolol was given. To further investigate whether anti-arrhythmic activity is accompanied by acceleration of ventricular tachycardia, verapamil $(0.5 \mathrm{mg} / \mathrm{kg}$ per 3 minutes) was given in 3 anesthetized dogs (figure 1).

\section{Hemodynamic experiments during idioventricular rhythm (protocol B)}

To characterize the relation between hemodynamics and rate after ryanodine, 6 anesthetized dogs were studied during idioventricular rhythm. Thirty minutes after the administration of a low dose of ouabain ${ }^{15}$ ( 1 minute bolus: $20 \mu \mathrm{g} / \mathrm{kg}$ ), insufficient to cause ventricular tachycardia, ryanodine $(10 \mu \mathrm{g} / \mathrm{kg}$ in 10 minutes $)$ was given.

\section{Recording of monophasic action potentials}

During ventricular tachycardia, the effect of ryanodine and verapamil on delayed afterdepolarizations was studied by recording monophasic action potential-signals in the anesthetized animals $(n=10)$. In the ryanodine experiments, endocardial monophasic action potential-signals were recorded in both ventricles with a quadripolar contact electrode $^{20}$ (Steerable Franz ${ }^{\mathrm{TM}}$ Combination Catheter, EP technologies). In the verapamil experiments, there was one monophasic action potential-signals recorded in the right ventricle. The catheters were randomly placed in the right and the left ventricle through the femoral or external jugular vein and the femoral artery respectively, with the 
aid of fluoroscopy. The monophasic action potential-signals were amplified using a custom built DC-coupled differential amplifier with a $20 \mathrm{mV}$ calibration pulse. The offset of this amplifier is variable and can be adjusted to the recorded signal. The monophasic action potential-signals are sampled at a rate of $1 \mathrm{kHz}$ per signal. For a detailed description of the methodology, we refer to a previous publication. ${ }^{21}$ The monophasic action potential-signals were checked for stability, a constant configuration and shape, and a minimally accepted amplitude of $15 \mathrm{mV}$ before the experiment started. ${ }^{20,21}$ Prior to the administration of any drug, the amplitude of the monophasic action potential-signal was checked. If the amplitude was found to be below $15 \mathrm{mV}$, the contact pressure of the signal was slightly increased, while maintaining the position and the configuration of the signal.

\section{Recording of left ventricular pressure signals}

To evaluate the effect of ryanodine, autonomic blockade and verapamil on left ventricular pressure and peak rate of pressure rise $(+\mathrm{LV} \mathrm{dP} / \mathrm{dt}$ max), the left ventricular pressure was measured with a single micro-manometer transducer catheter ( $7.5 \mathrm{~F}$, Sentron, Roden, The Netherlands). ${ }^{22,23}$ Prior to insertion, the pressure sensors were emerged in a $37^{\circ} \mathrm{C}$ $0.9 \%$ saline solution to minimize temperature drift. The Sentron interface (Type No $811-000 / \mathrm{A}$ ) was used for calibration of the pressure transducer. ${ }^{22,23}$ The pressure catheter was positioned in the left ventricle under fluoroscopic control via the carotid artery. The pressure signals were sampled at a rate of $1 \mathrm{kHz}$ per signal.

Left ventricular pressure curves were recorded 1) during ouabain induced ventricular tachycardia in the 3 dogs receiving verapamil and in 3 dogs receiving ryanodine and 2) in all dogs during idioventricular rhythm.

\section{Data analysis}

Using a software program, ${ }^{21}$ the data were analyzed off line. In protocol A, ventricular tachycardia cycle length (mean of 5 cycles) was measured every minute, from the moment of ventricular tachycardia induction until either ryanodine or verapamil was given. In the dogs receiving ryanodine, ventricular tachycardia cycle length was measured every minute from the start of ryanodine infusion $(t=0)$ until ventricular tachycardia termination. After the start of verapamil infusion, ventricular tachycardia cycle length was measured every 30 seconds. Ventricular tachycardia termination was defined as either complete termination or slowing down of the rhythm to a ventricular tachycardia cycle length of more than $600 \mathrm{~ms}$. In this protocol, the amplitude of the monophasic 

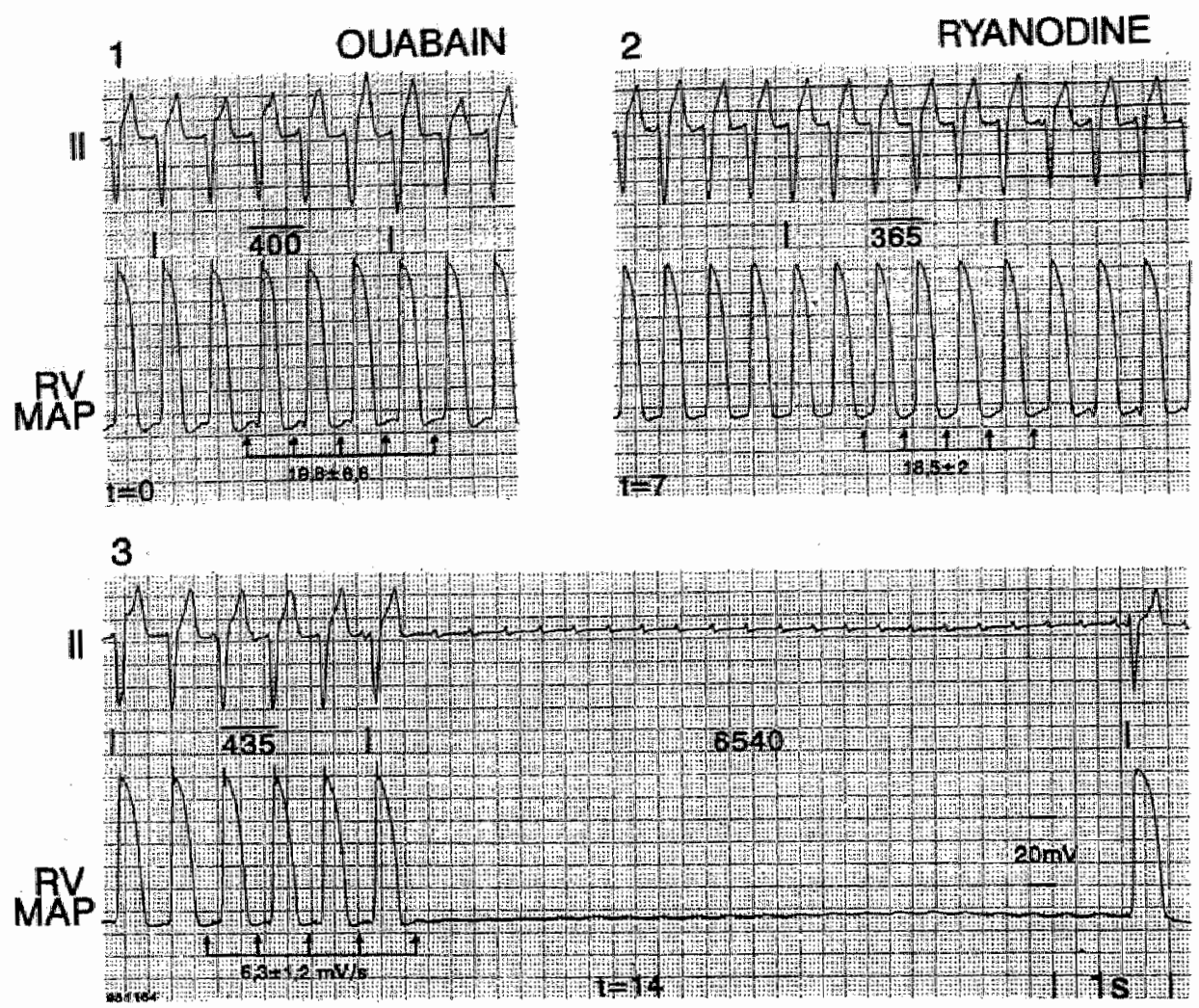

Figure 2: Termination of ouabain induced ventricular tachycardia (VT) with ryanodine in an anesthetized dog.

ECG lead II and a right ventricular (RV) monophasic action potential (MAP) recording are shown in each panel. Panel 1 shows the ouabain induced VT at the moment the ryanodine infusion is started (VT cycle length $400 \mathrm{~ms}$ ). The MAP shows a steep diastolic slope of $19.8 \pm 6.6 \mathrm{mV} / \mathrm{s}$. In panel 2, the VT is shown at the time of maximal rate acceleration (VT cycle length $365 \mathrm{~ms}$ ). At this moment, the slope in the MAP recording has already declined. Panel 3 shows VT termination 14 minutes after the start of ryanodine. Prior to termination, both the slope and the VT rate have declined.

action potential, the duration of the action potential at $100 \%$ repolarization, the diastolic interval (ventricular tachycardia cycle length - action potential duration, in ms), the slope $(\mathrm{mV} / \mathrm{s})$ of the diastolic interval of the monophasic action potential-recordings, the positive left ventriular $\mathrm{dP} / \mathrm{dt}(+\mathrm{LV} \mathrm{dP} / \mathrm{dt}: \mathrm{mm} \mathrm{Hg} / \mathrm{s})$ and left ventricular end systolic pressure $(\mathrm{mm} \mathrm{Hg})$ were measured at the same time points (every minute or every 30 seconds). Slope was calculated as the mean rate of rise (dV/dt) of the ascending limb of 
the delayed afterdepolarization, or as the mean value of diastolic rate of depolarization in between the paced or tachycardia beats. ${ }^{21}$ If hyperpolarization was present, this was excluded in the calculation of the slope. ${ }^{21}$ A delayed afterdepolarization was defined as a depolarizing afterpotential that begins after the complete repolarization. ${ }^{1,21}$

In protocol B, the cycle length of the idioventricular thythm was determined every minute from the start of the experiment until 10 minutes after ryanodine. At the same time, $+\mathrm{LV}$ $\mathrm{dP} / \mathrm{dt} \max$ and left ventricular systolic pressure were determined.

\section{Statistics}

All data are presented as mean \pm standard deviation (SD). To determine statistical significance, repeated measures analysis of variance (ANOVA) followed by Bonferroni's $t$-test was used for more than 2 group comparison. In the case of two-group comparison, paired students $t$-test was used.

\section{Results}

\section{Protocol A: experiments during ventricular tachycardia}

\section{Ryanodine administration in conscious dogs}

In the conscious dogs, ouabain administration and pacing resulted in ventricular tachycardia $99 \pm 54$ minutes after the start of ouabain. ventricular tachycardia cycle length was $358 \pm 52 \mathrm{~ms}$ at the moment of ryanodine infusion. Ryanodine terminated ventricular tachycardia in all 4 dogs, $12 \pm 1$ minutes after the start of the infusion. In this period ventricular tachycardia cycle length increased to $400 \pm 61 \mathrm{~ms}(\mathrm{p}<0.05)$. However, before slowing the tachycardia, ryanodine caused a temporary acceleration of the tachycardia rate in all 4 dogs, reaching a maximum at $6 \pm 1$ minutes after the start of the infusion (ventricular tachycardia cycle length $318 \pm 53 \mathrm{~ms}, \mathrm{p}<0.05$, compared to start of ryanodine).

\section{Ryanodine administration in anesthetized dogs}

Combining ouabain and pacing resulted in ventricular tachycardia after $48 \pm 38$ minutes. At the start of the ryanodine infusion ventricular tachycardia cycle length was $368 \pm 31$ $\mathrm{ms}(\mathrm{n}=7)$. Again, ryanodine terminated all ventricular tachycardias, $12 \pm 2$ minutes after the start of the infusion. As in the conscious dogs, ventricular tachycardia cycle length first decreased to a minimal length of $336 \pm 32 \mathrm{~ms}(p<0.05)$ after $7 \pm 2$ minutes of ryanodine infusion, before slowing of the rate and ventricular tachycardia termination occurred 


\section{Behavior of $+\mathrm{LV} d \mathrm{P} / \mathrm{dt}(-)$ and VT CL $(-\infty)$ during ryanodine after autonomic blockade}

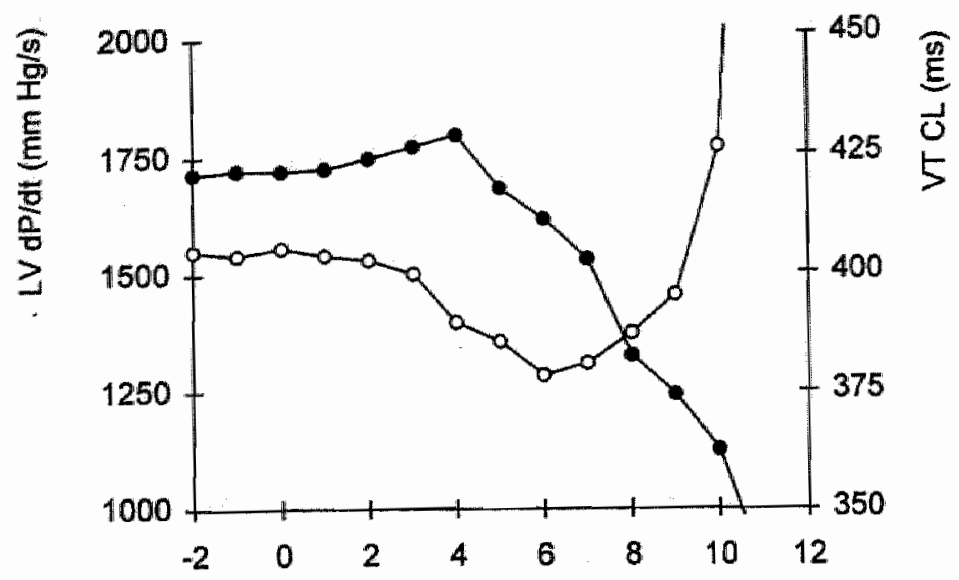

Time after start ryanodine (minutes)

Figure 3: Illustration of the behavior of $+\mathrm{LV} \mathrm{dP/dt}$ and ventricular tachycardia (VT) cycle length (CL) from the start of ryamodine infusion to VT termination.

$+\mathrm{LV} \mathrm{dP/dt}$ on the left $\mathrm{Y}$-axis, VT CL on the right $\mathrm{Y}$-axis and time after start of ryanodine on the $\mathrm{X}$-axis. As shown, after the start of the ryanodine infusion the VT rate shows acceleration followed by rate deceleration and VT termination. From the start of ryanodine to the termination of the VT the $+\mathrm{LV} d P / d t$ decreases from 1720 to $490 \mathrm{~mm} \mathrm{Hg} / \mathrm{s}$. Initially, a slight increase in $+L V \mathrm{dP} / \mathrm{dt}$ is observed, reaching its maximal value approximately 2 minutes before maximal VT acceleration is reached.

(ventricular tachycardia cycle length $421 \pm 32, \mathrm{p}<0.05$ compared to start of ryanodine, table 1, figure 2). This behavior of ventricular tachycardia cycle length was not affected by autonomic blockade 10-15 minutes before the administration of ryanodine ( $\mathrm{n}=2$ ). From the start of ryanodine infusion to maximal ventricular tachycardia acceleration, action potential duration showed no significant change (table 1). The length of the diastolic interval significantly shortened at the moment of maximal ventricular tachycardia acceleration: from $172 \pm 36$ to $147 \pm 31 \mathrm{~ms}$ for the left ventricle and from $18 \pm 23$ to $156 \pm 20 \mathrm{~ms}$ for the right ventricle ( $\mathrm{p}<0.05$ for both, table 1). Prior to ventricular tachy- 
Table 1: Ryanodine induced termination in anesthetized dogs

\begin{tabular}{lccc}
\hline & Start Ryanodine & $\begin{array}{c}\text { Accelaration } \\
\text { time }\end{array}$ & $\begin{array}{c}\text { Termination } \\
\text { time }\end{array}$ \\
\cline { 1 - 3 } Time (minutes) & 0 & $7 \pm 2$ & $12 \pm 2$ \\
CL (ms) & $368 \pm 33$ & $336 \pm 34^{*}$ & $421 \pm 75^{*}$ \\
LV APD (ms) & $196 \pm 21$ & $188 \pm 21$ & $199 \pm 17$ \\
RV APD (ms) & $186 \pm 22$ & $180 \pm 22$ & $186 \pm 25$ \\
Diastolic interval LV (ms) & $172 \pm 36$ & $147 \pm 31^{*}$ & $222 \pm 67^{* *}$ \\
Diastolic interval RV (ms) & $181 \pm 23$ & $156 \pm 20^{*}$ & $235 \pm 65^{* *}$ \\
LV slope (mV/s) & $19 \pm 12$ & $15 \pm 11$ & $8 \pm 4^{*}$ \\
RV slope (mV/s) & $22 \pm 17$ & $13 \pm 8$ & $5 \pm 4^{*}$ \\
\hline
\end{tabular}

*: $p<0.05$, compared to start of ryanodine infusion, **: $p<0.05$, compared to maximal acceleration, $\mathrm{CL}$ : cycle length in $\mathrm{ms}, \mathrm{LV}$ : left ventricle, RV: right ventricle, APD: action potential duration

cardia termination, the diastolic interval prolonged in both ventricles (table 1). The left ventricular slope was $19 \pm 12 \mathrm{mV} / \mathrm{s}$ and the right ventricular slope was $22 \pm 17 \mathrm{mV} / \mathrm{s}$ at the start of the ryanodine infusion. Just before termination, both the left and right ventricular slope decreased significantly to $8 \pm 4$ and $5 \pm 4 \mathrm{mV} / \mathrm{s}$ respectively (table 1 ). At the moment of the shortest ventricular tachycardia cycle length, slope showed no significant change in either ventricle (figure 2), although a tendency to decrease was present indicating a different time dependency of slope and rate.

$+\mathrm{LV} \mathrm{dP} / \mathrm{dt}, 2016 \pm 392 \mathrm{~mm} \mathrm{Hg} / \mathrm{s}$ at the start of ryanodine infusion, decreased to $1576 \pm 55$ $\mathrm{mm} \mathrm{Hg} / \mathrm{s}$ at the time of the maximal ventricular tachycardia rate and further decreased to $1106 \pm 225 \mathrm{~mm} \mathrm{Hg} / \mathrm{s}$. Simultaneously, left ventricular systolic pressure decreased from $116 \pm 23$ to $98 \pm 2$ and to $90 \pm 12 \mathrm{~mm} \mathrm{Hg}$ at the moment of ventricular tachycardia termination.

\section{Synchronicity of the observed changes}

The time-dependent behavior of some parameters does not seem to be synchronized; based on the assumed agonistic effect of ryanodine on sarcoplasmic reticulum calcium release, we expected to find a maximal increase in $+\mathrm{LV} \mathrm{dP} / \mathrm{dt}$ and in diastolic slope of the delayed afterdepolarization at the moment of maximal ventricular tachycardia 

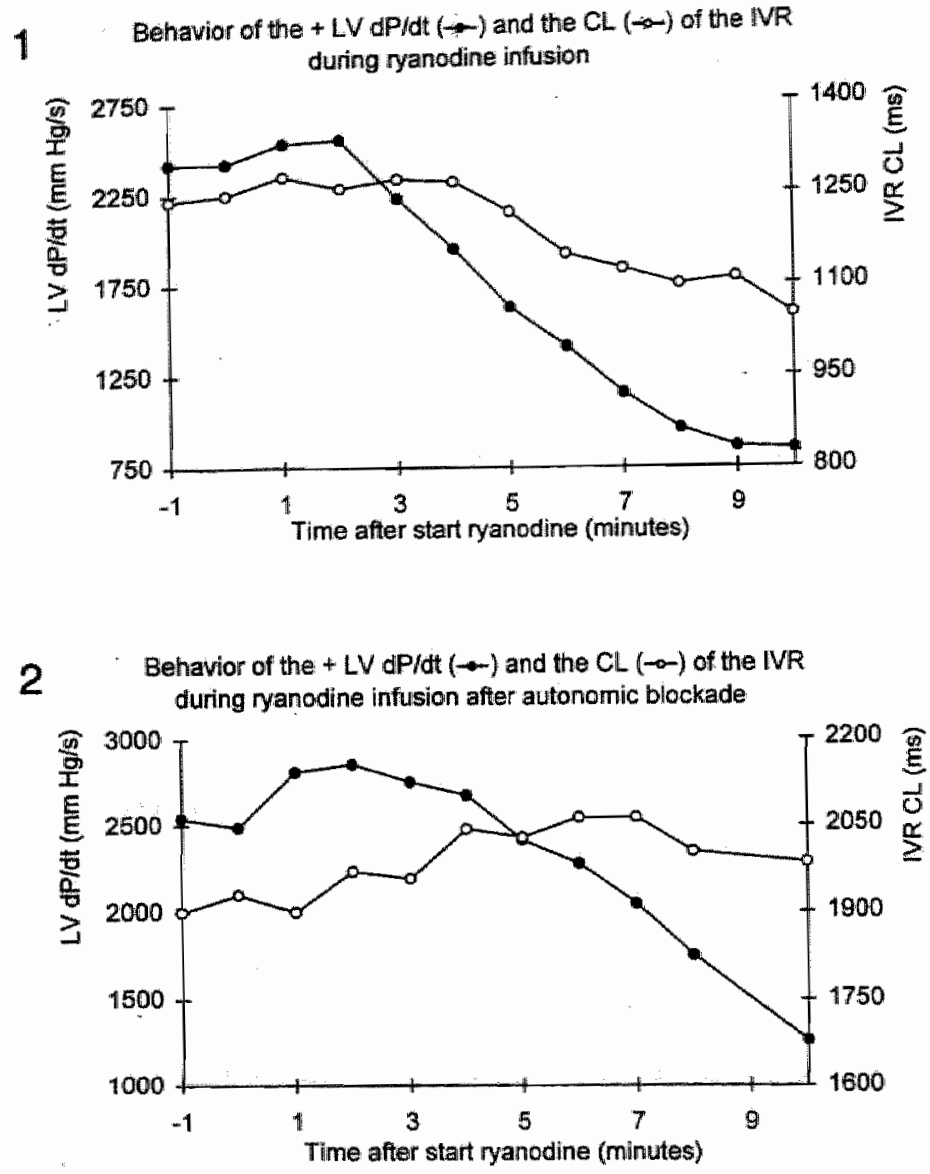

Figure 4: Behavior of the cycle length $(\mathrm{CL})$ and $+\mathrm{LV} \mathrm{dP} / \mathrm{dt}$ after ryanodine administration during idioventricular rhythm (IVR), without (panel 1) and with (panel 2) autonomic blockade.

Both panels have an identical composition as figure 3.

Panel 1: After the start of ryanodine infusion, the CL of the IVR starts to shorten after approximately 5 minutes until a CL of $1050 \mathrm{~ms}$ is reached after 10 minutes of ryanodine. During this time span, ryanodine exerts a negative inotropic effect reflected in the decreased $+\mathrm{LV} \mathrm{dP} / \mathrm{dt}$ (from 2424 to $850 \mathrm{~mm} \mathrm{Hg} / \mathrm{s}$ ). However, before a decrease in $+\mathrm{LV} \mathrm{dP} / \mathrm{dt}$ is observed, a positive inotropic effect is seen, reaching its maximum 2 minites after the start of ryanodine.

Panel 2. When ryanodine is given after autonomic blockade, the CL of the IVR does not change. However, the response of the $+\mathrm{LV} \mathrm{dP} / \mathrm{dt}$ initially shows a positive inotropic effect, before the negative inotropic action of ryanodine becomes manifest. 
acceleration. However, there was no change in the diastolic slope of the delayed afterdepolarization and a decreased $+\mathrm{LV} \mathrm{dP/dt}$. Therefore, we measured the different parameters in time. For ventricular tachycardia cycle length and $+\mathrm{LV} \mathrm{dP} / \mathrm{dt}$, this behavior is illustrated in figure 3 for one individual dog after the start of ryanodine infusion. In this example, ryanodine is given after autonomic blockade. As can be seen from this figure, the $+\mathrm{LV} \mathrm{dP} / \mathrm{dt}$ shows a tendency to increase during the first 4 minutes of ryanodine infusion. Afterwards, the $+\mathrm{LV} \mathrm{dP} / \mathrm{dt}$ declines, with a minimal value of $490 \mathrm{~mm} \mathrm{Hg} / \mathrm{s}$ for the first contraction after ventricular tachycardia termination. In the same time span, ventricular tachycardia cycle length behaves differently. During the first 4 minutes of ryanodine infusion, ventricular tachycardia cycle length decreases, with the fastest heart rates occurring after 6 minutes of ryanodine infusion. Thereafter, ventricular tachycardia cycle length starts to increase and ventricular tachycardia terminates after 10 minutes of ryanodine.

\section{Verapamil administration in anesthetized dogs}

At a ventricular tachycardia duration of $20 \pm 5$ minutes, administration of verapamil resulted in termination after $127 \pm 43$ seconds. In this time period, cycle length increased from $368 \pm 28 \mathrm{~ms}$ at the start of verapamil infusion to $532 \pm 55 \mathrm{~ms}$ at the moment of ventricular tachycardia termination $(p<0.05)$. No acceleration of ventricular tachycardia rate was seen. Synchronous with the slowing in ventricular tachycardia rate, left ventricular pressure and the diastolic slope of the delayed afterdepolarizations decreased. Left ventricular systolic pressure, $+\mathrm{LV} \mathrm{dP/dt}$ and the right ventricular slope decreased from $108 \pm 14$ to $85 \pm 17 \mathrm{~mm} \mathrm{Hg}, 2622 \pm 680$ to $1968 \pm 694 \mathrm{~mm} \mathrm{Hg} / \mathrm{s}$, and $16 \pm 5$ to $6 \pm 3 \mathrm{mV} / \mathrm{s}$ respectively $(\mathrm{p}<0.05$, for all).

\section{Protocol B: Effeet of ryanodine during idioventricular rhythm}

In all dogs $(n=6)$, the cycle length of the idioventricular rhythm remained stable during the control period $(1483 \pm 470 \mathrm{~ms})$ and after a low dose of ouabain (1398 $\pm 443 \mathrm{~ms}$ ). Ouabain non-significantly increased left ventricular systolic pressure (from $107 \pm 8$ to $115 \pm 8 \mathrm{~mm} \mathrm{Hg}$ ) and increased the $+\mathrm{LV} \mathrm{dP} / \mathrm{dt}$ from $2348 \pm 223$ to $2648 \pm 271 \mathrm{~mm} \mathrm{Hg} / \mathrm{s}$ $(\mathrm{p}<0.05)$. Autonomic blockade $(\mathrm{n}=3)$ resulted in marked slowing of the idioventricular rhythm, with an increase in cycle length from $1943 \pm 678$ to $3607 \pm 2324 \mathrm{~ms}$ in 12 minutes $(\mathrm{p}<0.05$ ). This was accompanied by a significant decrease of the $+\mathrm{LV} \mathrm{dP} / \mathrm{dt}$ from $3136 \pm 45$ to $2416 \pm 192 \mathrm{~mm} \mathrm{Hg} / \mathrm{s}$. 
Administration of ryanodine without autonomic blockade resulted ultimately (after 4-6

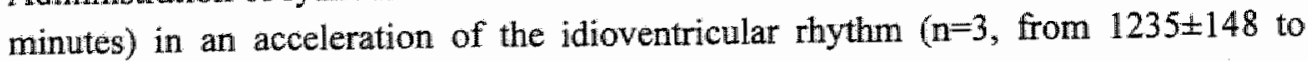
$1094 \pm 197 \mathrm{~ms}, \mathrm{p}<0.05$ ) and a decrease in $+\mathrm{LV} \mathrm{dP} / \mathrm{dt}$ (from $2446 \pm 29$ to $14.12 \pm 409 \mathrm{~mm}$ $\mathrm{Hg} / \mathrm{s}, \mathrm{p}<0.05$ ) (illustrated for an individual dog in figure 4, panel 1). By giving ryanodine after autonomic blockade, the rate acceleration was prevented, but the negative inotropic effect on cardiac contractile function was maintained (illustrated for an individual dog in figure 4, panel 2).

However, independent of the presence or absence of autonomic blockade, ryanodine administration resulted in an initial increase in left ventricular contractile function; after $105 \pm 25$ seconds of ryanodine infusion the $+\mathrm{LV} \mathrm{dP} / \mathrm{dt}$ had increased from $2366 \pm 226$ to $2617 \pm 146 \mathrm{~mm} \mathrm{Hg} / \mathrm{s}(\mathrm{p}<0.05)$. In figure 4 , this behavior of the positive left ventricular $\mathrm{dP} / \mathrm{dt}$ is illustrated for $2 \mathrm{dogs}$, one dog with and one dog without autonomic blockade.

\section{Discussion}

As expected, ryanodine terminated ouabain-induced ventricular tachycardia. To our surprise, we found that rate slowing and ventricular tachycardia termination is preceded by a rate acceleration. We provide evidence that this rate acceleration is most likely caused by an agonistic effect of ryanodine on sarcoplasmic reticulum calcium release and not by a sympathetically mediated reflex mechanism because of a negative inotropic effect of ryanodine. These observations provide direct evidence for a relation between functional status of the myocardium and delayed afterdepolarization-dependent ventricular tachycardias.

\section{Model}

The animal model of ouabain-induced ventricular tachycardias used in this study, has previously been studied in both conscious and anesthetized dogs. ${ }^{3,21,24,25}$ The induction of these arrhythmias is facilitated by combining ouabain administration with ventricular pacing. ${ }^{15,24,26}$ At subtoxic levels of ouabain intoxication, ventricular ectopic beats can be induced. With progression of intoxication, they increase in number and shorten their coupling interval until ventricular tachycardia occurs. ${ }^{15,25}$ In the canine heart, the induction of both ventricular tachycardia and ectopic beats during ouabain results from triggered activity based on delayed afterdepolarizations. ${ }^{3,21,25}$ Delayed afterdepolarizations occur in the setting of intracellular calcium overload, causing the sarcoplasmic reticulum to spontaneous oscillatory release of $\mathrm{Ca}^{2+}$ in the cytosol, thereby eliciting an transient inward current $\left(\mathbb{I}_{\mathrm{ti}}\right)$ which is responsible for the delayed afterdepolarizations. ${ }^{27-}$ 
${ }^{30}$ Proposed mechanisms underlying the $\mathrm{Iti}_{\mathrm{ti}}$ are inverse $\mathrm{Na}^{+} / \mathrm{Ca}^{2+}$ exchange, a calcium activated non specific cation current, or a calcium-induced chloride current. ${ }^{27-30} \mathrm{By}$ blockade of the sarcoplasmic calcium release channel, ryanodine will suppress delayed afterdepolarizations and cause ventricular tachycardia termination. ${ }^{31}$

\section{Conscious versus anesthetized dogs}

In the preliminary experiments using conscious dogs, ryanodine caused a ventricular tachycardia rate acceleration before slowing of the rate and ventricular tachycardia termination. This acceleration in rate had not been previously observed in our model with other anti-arrhythmic drugs like lidocaine, flunarizine, magnesium, $R 56865$, morizicine and verapamil. ${ }^{3-6}$ Ouabain induced ventricular tachycardias are delayed afterdepolarization-dependent, ${ }^{15,32}$ and delayed afterdepolarizations can be registered in vivo with monophasic action potentials, ${ }^{4,21,33}$ We first tried to elucidate the cause of this rate acceleration by studying delayed afterdepolarization behavior. In order to introduce the monophasic actionpotential -catheters, general anesthesia was required, otherwise the model remained the same. By combining an identical dosage of ouabain with pacing, similar ventricular tachycardias were induced in both the conscious and the anesthetized dogs, although ventricular tachycardias were induced slightly earlier in time during anesthesia. Despite the use of anesthetics, ryanodine administration resulted in (1) a similar shortening of ventricular tachycardia cycle length in both the conscious and anesthetized animals ( $40 \mathrm{~ms}$ in 6 minutes, versus $32 \mathrm{~ms}$ in 7 minutes respectively); (2) a similar increase in ventricular tachycardia cycle length before (3) ventricular tachycardia termination occurred at the same time after the start of ryanodine.

\section{Sympathetically mediated reflex mechanism}

Ryanodine has been described to exert a pronounced negative inotropic effect. ${ }^{7,8,34}$ This is confirmed in our experiments. Because we noticed that the negative inotropic effect was already present at the moment of maximal ventricular tachycardia acceleration, we hypothesized that ventricular tachycardia acceleration was caused by a reflex mediated sympathetic mechanism. Previously, we have noticed such an acceleration of ouabain induced ventricular tachycardia after administration of the IK-ATP activator levcromakalim which causes hypotension. ${ }^{5}$ In those experiments, propranolol administration prior to levcromakalim prevented the acceleration in ventricular tachycardia rate. ${ }^{5}$ Autonomic blockade in the ryanodine experiments did not affect the transient rate acceleration of the ouabain induced ventricular tachycardia. Additionally, in the verapamil experiments, 
no acceleration of ventricular tachycardia rate was observed, despite a negative inotropic action. These observations make a reflex mediated sympathetic cause of the rate acceleration unlikely and suggest another effect of ryanodine.

\section{Behavior of the diastolic slope of the delayed afterdepolarization}

Although monophasic action potential-recordings are not identical to transmembrane action potentials, they can be used for the detection of afterdepolarizations in vivo. ${ }^{35} \mathrm{We}$ tried to study the influence of ryanodine on delayed afterdepolarizations by quantifying the behavior of the ventricular tachycardia cycle length and the diastolic slope of the delayed afterdepolarizations. One would expect that the amplitude or steepness of the slope of the delayed afterdepolarizations is related to the ventricular tachycardia cycle length, because a decrease in these parameters leads to slowing in ventricular tachycardia rate and ventricular tachycardia termination. 1,32,36,37

For the first time, our data show a steep diastolic slope of the monophasic action potential of both the left and the right ventricle during ouabain induced ventricular tachycardia, which decreases towards ryanodine induced ventricular tachycardia termination. This corresponds with observations made in isolated tissue preparations, where, during sustained triggered activity, the delayed afterdepolarization that triggers an action potential is only visible as a steep diastolic slope. ${ }^{32}$ Towards termination of the spontaneous activity, the diastolic slope declines and the coupling interval prolongs. ${ }^{1,32}$ Probably, this effect can be attributed to blockade of the calcium release channel of the sarcoplasmic reticulum by ryanodine. ${ }^{31,34}$

Towards termination of ouabain induced ventricular tachycardia, the behavior of the delayed afterdepolarizations, quantified by the diastolic slope, corresponds in accordance with the behavior of ventricular tachycardia rate. However, this is not the case at the moment of maximal ventricular tachycardia acceleration, when the diastolic slope of the delayed afterdepolarization shows a tendency to decrease. At no time during the ryanodine infusion, we were able to detect a significant increase in the slope or the amplitude of the delayed afterdepolarization, to explain the rate acceleration. A possible explanation could be that the slope was measured in randomly placed monophasic action potential-recordings, without considering the site of delayed afterdepolarization origin in relation to the site of recording. The shift in ventricular tachycardia focus that is sometimes seen, supports this. Although we found a steep slope and delayed afterdepolarizations in both the left and right ventricular monophasic action-registrations at the start of ryanodine, we do not know to what extent our registration of delayed afterdepo- 
larizations and slope are a true manifestation of the exact site of origin. Other experiments are necessary to study this. Other possible explanations might be found in changes of maximum diastolic membrane potential, or of threshold.

\section{Left ventricular pressure}

By recording of the left ventricular pressure and by determining the positive left ventricular $\mathrm{dP} / \mathrm{dt}$, we confirmed the negative inotropic effect of ryanodine. $7,8,12,34$ Besides this negative inotropic effect, we also noticed an initial positive inotropic effect during ryanodine infusion. Because of the limited number of dogs with ouabain induced ventricular tachycardia in which a left ventricular pressure signal was recorded, we extended this number by giving ryanodine during idioventricular rhythm after a therapeutic dose of ouabain. Also in these experiments an initial positive inotropic effect was found, suggesting an initial agonistic instead of antagonistic (blocking) effect of ryanodine on sarcoplasmic reticulum calcium release.

\section{Rate acceleration during ouabain induced ventricular tachycardia versus idioventricular rhythm}

In contrast to the rate of the idioventricular rhythm, the rate of the delayed afterdepolarization-dependent ouabain induced ventricular tachycardia is influenced by alterations in sarcoplasmic reticulum calcium release. ${ }^{28}$ Therefore, we hypothesized that if ryanodine truly exerts an agonistic effect on sarcoplasmic reticulum calcium release, this would only affect the rate of the ouabain induced ventricular tachycardia and not of the idioventricular rhythm. However, ryanodine accelerated the idioventricular heart rate. Autonomic blockade prevented this acceleration, but the ryanodine induced rate acceleration during ouabain induced ventricular tachycardia was not prevented. Thus, the ryanodine induced rate acceleration during idioventricular rhythm seems to be caused indirectly by a reflex mediated sympathetic mechanism, while during ventricular tachycardia this seems a direct consequence of ryanodine infusion.

\section{Agonistic effect of ryanodine on ouabain induced ventricular tachycardias}

In summary, a direct effect of ryanodine on sarcoplasmic reticulum calcium release is suggested to play a role in the observed ventricular tachycardia rate acceleration, since 1) ventricular tachycardia acceleration persists during autonomic blockade, 2) an initial positive inotropic effect is observed before the eventual negative inotropic effect, and 3 ) the absence of a direct effect of ryanodine on the idioventricular heart rate which is not influenced by sarcoplasmic reticulum calcium handling. 
The findings in our study could be explained as follows: ryanodine given in a dose of 10 $\mu \mathrm{g} / \mathrm{kg}$ is effective in suppressing cardiac glycoside induced arrhythmias. ${ }^{7,8}$ Infusion has to be slow enough to prevent death of the animal. ${ }^{8,14}$ However, the slow rate of ryanodine infusion will cause a relatively long period with a low plasma concentration of ryanodine, which may exert an agonistic effect on $\mathrm{Ca}^{2+}$ release instead of an antagonistic effect. $7,8,34,38$ In the literature, this hypothesis seems to be supported by 1) the positive inotropic effect of low dosages of ryanodine and a negative inotropic response with high dosages of ryanodine when studied in isolated tissue preparations, $11,12,392$ ) an increase of cesium induced ectopic activity after ryanodine ${ }^{13}$ and 3) the spontaneous occurrence of Torsade de Pointes arrhythmias after d-sotalol ${ }^{14}$ at low concentrations of ryanodine. In line with this explanation the effect of ryanodine at the channel level can be mentioned. Channel behavior is affected in such a way that the conductance shifts to a substrate (antagonistic effect), but at the same time the channel stays open for longer times (agonistic effect). This finally causes emptying of the sarcoplasmic reticulum. ${ }^{38}$ Although the antagonistic effect of ryanodine on delayed afterdepolarizations and related triggered activity and cardiac contractile function has been extensively studied, ${ }^{7-9}$ we are not aware of studies which indicate that an agonistic effect of ryanodine on calcium release favors delayed afterdepolarization-dependent arrhythmias in the intact heart. Additionally, the combinated recording of both monophasic action potentials and left ventricular pressure curves to evaluate calcium related arrhythmias has not been used previously.

\section{Limitations}

In the intact dogs used in this study, we speculate on modulating intracellular calcium with both ouabain and ryanodine, but we are not able to measure calcium transients to prove changes in intracellular calcium.

The observed acceleration of ventricular tachycardia rate was not affected by autonomic blockade and seems therefore not related to a reflex mechanism. However, interpretation of these indirect data has to be performed with caution. For example, a contribution of the $\alpha$-adrenergic system to the observed effect cannot be excluded.

\section{Conclusions}

The results of this study indicate that ryanodine effectively terminates ouabain induced ventricular tachycardia in both conscious and anesthetized dogs, probably because of blockade of the sarcoplasmic calcium release channel. However, this antagonistic effect 
of ryanodine on calcium release is preceded by a agonistic effect, causing both a positive inotropic effect and a pro-arrhythmic effect on calcium related delayed afterdepolarization-dependent ouabain induced ventricular tachycardia.

\section{Acknowledgments}

This study was supported by a grant from the Wynand N. Pon Foundation, Leusden, The Netherlands. The authors would like to thank the Bakken Research Center, Maastricht, The Netherlands for supplying the electrodes and the Netherlands Organization for Scientific Research for providing a travel grant.

\section{References}

1. Cranefield PF, Aronson R. Cardiac arrhythmias: The role of triggered activity and other mechanisms. New York.: Mt Kisco, New York, Futura Publishing Co., 1988.

2. Lakatta EG. Functional implications of spontaneous sarcoplasmic reticulum $\mathrm{Ca}^{2+}$ release in the heart. Cardiovasc Res 1992; 26: 193-214.

3. Vos MA, Gorgels AP, Leunissen JD, Wellens HJ. Flunarizine allows differentiation between mechanisms of arrhythmias in the intact heart. Circulation 1990; 81: 343-349.

4. Vos MA, Fazekas T, Gorgels AP, Leunissen JD, Wellens $\mathrm{HI}$. Action of MgSO 4 differs from moricizine and verapamil on ouabain-induced ventricular tachycardia in normomagnesemic conscious dogs. J Cardlovasc Pharmacol 1994; 23:252-258.

5. Vos MA, Gorgels AP, Lipesei GC, De Groot SH, Leunissen JD, Wellens HJ. Mechanism-specific antiarrhythmic effects of the potassium channel activator levcromakalim against repolarization-dependent tachycardias. J Cardiovasc Electrophysiol 1994; 5:731-742.

6. Vos MA, van Deursen RT, Gorgels AP, Leunissen JD, Wellens HJ. R56865, an antiarrhythmic drug with class III effects that terminates ouabain induced ventricular tachycardia in an inverse rate dependent manner. Cardiovase Res $1993 ; 27: 1491-1497$.

7. Kahn M, Shiffman I, Kuhn LA, Jacobson TE. Effects of ryanodine in normal dogs and those with digitalis-induced arrhythmias. $A m J$ Card $1964 ; 14: 658-668$.

8. Hadju S, Leonard E. Action of ryanodine on mammalian cardiac muscle. Effects on contractility, and reversal of digitalis induced ventricular arrhythmias. Circ Res 1961; 9:1291-1298.

9. Marban E, Robinson SW, Wier WG. Mechanisms of arrhythmogenic delayed and early afterdepolarizations in ferret ventricular muscle. J Clin Invest 1986; 78: 1.185-1192.

10. Hashímoto K, Haruno A, Matsuzaki T, Sugiyama A, Akiyama $K$. Effects of antiarrhythmic drugs on canine ventricular arrhythmia models: which electrophysiological characteristics of drugs are related to their effectiveness. Cardiovasc Drug Ther 1991; 5: 805-818.

11. Sutko $\mathrm{L}$, Willerson JT. Ryanodine alteration of the contractile state of rat ventricular myocardium. Comparison with dog, cat, and rabbit ventricular tissues. Circ Res 1980;46:332-343.

12. Zakharov SI, Bogdanov KY, Golovina VA. Effects of ryanodine on ouabain-induced spontaneous mechanical and electrical oscillations in guinea-pig heart. $J \mathrm{Mol}$ Cell Cardiol 1991; 23:41-46.

13. D'Alonzo AJ, Hess TA, Darbenzio RB, Sewter JC. Effects of intracoronary glyburide on cesium induced arrhythmias in the anaesthetized dogs. J Cardiovasc Pharmacol 1994; $23: 446-452$. 
14. Verduyn SC, Vos MA, Gorgels AP, van der Zande J, Leunissen JD, Wellens HJ. The effect of flunarizine and ryanodine on acquired torsades de pointes arrhythmias in the intact canine heart. $J$ Cardiovasc Electrophysiol 1995; 6: 189-200.

1.5. Vos MA, Gorgels AP, Leunissen JD, van Deursen RT, Wellens HJ. Significance of the number of stimuli to initiate ouabain-induced arrhythmias in the intact heart. Circ Res $1991 ; 68: 38-44$.

16. Scherlag BJ, Kosowsky BD, Damato AN. Technique for ventricular pacing from the His bundle of the intact heart. $J$ Appi Physiol 1967; 22: 584-587.

17. Gorgels AP, De Wit B, Beekman HD, Dassen WR, Wellens HJ. Triggered activity induced by pacing during digitalis intoxication observations during programmed electrical stimulation in the conscious dog with elhronic complete atrioventricular block. PACE 1987; 10: 1309-1321.

18. Vos MA, Gorgels AP, Leun issen JD, Wellens HJJ. The in vivo response of ouabain-induced arrhythmias to pacing: Acceleration instead of termination. Am Heart $J 1990 ; 120: 604-61 \mathrm{l}$.

19. Talajic $M_{3}$ Nattel $S$. Frequency-dependent effects of calcium antagonists on atrioventricular conduction and refractoriness. Demonstration and characterization in anaesthetized dogs. Circulation 1986: 74: 1156-1167.

20. Franz MR, Chin MC, Sharkey HR, Griffin JC, Scheinman MM. A new single catheter technique for simultaneous measurement of action potential duration and refractory period in vivo. $J \mathrm{Am}$ Coll Cardiol 1990; 16: 878-886.

21. De Groot SHM, Vos MA, Gorgels APM, Leunissen JDM, Van der Steld BJ, Wellens HJJ. Combining monophasic action potential recordings with pacing to demonstrate delayed afterdepolarizations and triggered arrhythmias in the intact heart. Circulation 1995; 92: 2697-2704.

22. Aubert AE. In vivo comparison between two tip pressure transducer systems. Int J Clin Monitor Computing 1995; 12: 77-83.

23. Iledema $\mathbf{P}$, Bakkes DJF. Pressure measurement in the cardiac catheterization laboratory" avoiding essential errors. Bilthoven, The Netherlands: Viggo-Spectramed BV, 1990.

24. Gorgels $A \mathrm{AP}_{3}$ Vos MA, Brugada $\mathrm{P}$, Wellens HJJ. The clinical relevance of abnormal automaticity and triggered activity. In: Brugada $\mathrm{P}$, Wellens $\mathrm{HJJ}$, eds. Cardiac arrhytmias: Where to go from here? New York: Futura Publishing Company, Inc., 1987: 147-169.

25. Hagemeijer F, Lown B. Effect of heart rate on electrically induced repetitive ventricular responses in the digitalized dog. Circ Res 1970; 27: 333-344.

26. Wittenberg SM, Streuli F, KJocke FJ. Acceleration of ventricular pacemakers by transient increases in heart rate in dogs during ouabain administration. Circ Res $1970 ; 26: 705-716$.

27. Smith TW. Digitalis. Mechanisms of action and clinical use. $N$ Engl J Med 1988; 318:358-365.

28. Rosen MR. Cardiac arrinythmias and antiarrhythmic drugs: recent advances in our understanding of mechanism. J Cardiovasc Electrophysiol 1995; 6: 868-879.

29. Fedida D, Noble D, Rankin AC, Spindler AJ. The arrhythmogenic transient inward current Iti and related contraction in isolated guinea-pig ventricular myocytes. J Physiol (Lond) 1987; 392: 523 542.

30. Berlin JR, Cannell MB, Lederer WJ. Cellular origins of the transient inward current in cardiac myocytes. Role of fluctuations and waves of elevated intracellular calcium. Circ Res 1989; 65:115-126.

31. Marban $\mathbb{E}$, Wier WG. Ryanodine as a tool to determine the contributions of calcium entry and calcium release to the calcium transient and contraction of cardiac Purkinje fibers. Circ Res $1985 ; 56$ : $133-138$.

32. Le Marec $\mathrm{H}_{\text {; }}$ Spinelli $\mathrm{W}$, Rosen MR. The effects of doxorubicin on ventricular tachycardia. Circulation 1986; 74: 881-889. 
33. Furukawa T, Kimura S, Castellanos A, Bassett AL, Myerburg RJ. In vivo induction of "focal" triggered ventricular arrhythmias and responses to overdrive pacing in the canine heart. Circulation $1990 ; 82: 549-559$.

34. Sutko JL, Willerson JT, Templeton GH, Jones LR, Besch HR Jr. Ryanodine: its alterations of cat papillary muscle contractile state and responsiveness to inotropic interventions and a suggested mechanism of action. J Pharmacol Exp Ther 1979; 209:37-47.

35. Franz MR. Bridging the gap between basic and clinical electrophysiology: What can be learned from monophasic action potential recordings. J Cardiovasc Electrophysiol 1994; 5: 699-710.

36. Ferrier GR, Saunders JH, Mendez C. A cellular mechanism for the generation of ventricular arrhythmias by acetylstrophanthidin. Circ Res $1973 ; 32: 600-609$.

37. Rosen MR, Gelband H, Merker $C$, Hoffman BF. Mechanisms of digitalis toxicity. Effects of ouabain on phase four of canine Purkinje fiber transmembrane potentials. Circulation 1973;47: 681-689.

38. Meissmer $G$, Henderson JS. Rapid calcium release from cardiac sarcoplasmic reticulum vesicles is dependent on $\mathrm{Ca}^{2+}$ and is modulated by $\mathrm{Mg}^{2+}$, adenine nucleotide, and calmodulin.JBiol Chem $1987 ; 262: 3065-3073$.

39. Saxon ME, Kobrinski EM. Ryanodine in low concentrations is a Ca-release stimulator rather than inhibitor in rat myocardium. Gen Physiol Biophys 1988; 7: 39-49. 


\title{
Chapter 5
}

\section{Enhanced susceptibility for acquired Torsade de Pointes arrhythmias in the dog with chronic, complete atrioventricular-block is related to cardiac hypertrophy and electrical remodeling}

\author{
Marc Vos \\ Marieke de Groot \\ Cora Verduyn \\ Jolanda van der Zande \\ Jet Leunissen \\ Jack Cleutjens \\ Marc van Bilsen \\ Mat Daemen \\ Jan Schreuder \\ Maurits Allessie \\ Hein Wellens
}

Abstracts presented at the American Heart Association 1995 (Circulation 1995; 92 : 1504-1505) and at the European Society of Cardiology 1996 (Eur Heart J 1996; 17: 549) Accepted for publication in Circulation 


\section{Abstract}

Introduction: Left ventricular hypertrophy is an independent risk factor for ventricular arrhythmias and sudden death. It is known that the chronic atrioventricular-block dog develops ventricular hypertrophy. This dog model has been used by us to investigate acquired Torsade de Pointes arrhythmias. It was the purpose of this study to describe the short and long term electrical adaptations that occur in this dog after atrioventricularblock and to investigate whether these adaptations predispose the heart for Torsade de Pointes. Secondly, we wanted to gain more insight in the nature of the structural and functional adaptations involved.

Methods and Results: We determined 1) the duration of the endocardial right and left ventricular action potential duration), the $\triangle \mathrm{APD}$ (left-right ventricular action potential duration), and the presence of early afterdepolarizations at 0 (acute) and 6 (chronic) weeks of atrioventricular-block and related these electrophysiological findings to the ability to induce acquired Torsade de Pointes by pacing $(n=10), 2)$ structural parameters of the left and right ventricle of atrioventricular-block dogs $(10 \pm 2$ weeks, $n=6)$ compared to sinus rhythm dogs $(n=6), 3)$ expression of ventricular mRNA Atrial Natriuretic Factor (ANF) in atrioventricular-block and sinus-rhythm dogs, 4) plasma neurohumoral levels at different time intervals $(n=7), 5)$ left ventricular hemodynamics at 0 and 6 weeks $(n=6)$. In comparison to acute atrioventricular-block, chronic atrioventricular-block led to a) a non-homogeneous prolongation of left and right ventricular action potential duration, and b) a different sensitivity for $d$-sotalol leading to the frequent occurrence of early afterdepolarizations ( $4 / 14$ versus $9 / 18, p<0.05$ ), increased $\triangle A P D$ ( $45 \pm 30$ versus $125 \pm 65$ $\mathrm{ms}, \mathrm{p}<0.05)$ and to the ability to induce Torsade de Pointes in the majority of the dogs $(0 \%$ versus $60 \%, \mathrm{p}<0.05)$. At autopsy, the atrioventricular-block dogs showed biventricular hypertrophy. The left and right ventricular collagen fraction and the capillary to fiber ratio remained normal, while the levels of ANF mRNA were not detectable. The neurohumoral plasma levels were elevated only transiently. Left ventricular function was similar at acute and chronic atrioventricular-block.

Conclusions: The electrical remodeling occurring after chronic atrioventricular-block predispose the heart for acquired Torsade de Pointes, while the structural changes (hypertrophy) are successfully recruited to maintain cardiac function. 


\section{Introduction}

For years we have been using the chronic, complete atrioventricular-block dog to investigate mechanisms of cardiac arrhythmias with emphasis on triggered arrhythmias. ${ }^{1-5}$ Initially, we delayed the first experiment on the inducibility of ventricular tachycardias till at least two weeks after creation of atrioventricular-block to allow the animal sufficient time to adapt. In time, the chronic atrioventricular-block dog develops ventricular hypertrophy presumably on the basis of volume overload. ${ }^{6-8}$ Many studies have indicated that a link may exist between arrhythmias and hypertrophy. Epidemiological studies indicate that the incidence of sudden death is greater in patients with myocardial hypertrophy than in the normal population. The Framingham study for instance, reported almost a sixfold increase in sudden cardiac death in hypertensive man with left ventricular hypertrophy. 9,10 This higher incidence of ventricular arrhythmias is independent of other factors like coexisting coronary artery disease and/or heart failure. ${ }^{11,12}$ Alterations in electrophysiological properties of the ventricular myocardium due to this hypertrophic response may underlie this increased susceptibility.

Evidence about electrical remodeling due to hypertrophy has been derived from animal models. ${ }^{13-20}$ One of the most consisting findings in isolated hypertrophied myocardium is prolongation of the action potential, which seems independent of the cause of hypertrophy. It has been suggested that due to this lengthening of repolarization, early afterdepolarizations and triggered activity may occur in hypertrophy and one may assume that early afterdepolarization related Torsade de Pointes arrhythmias with the possibility of deterioration into ventricular fibrillation could be a possible cause of sudden death. ${ }^{18-}$ 20 Recently, we have described that interventricular dispersion of repolarization also plays a key role in the induction of acquired Torsade de Pointes ${ }^{5}$ in the dog with chronic atrioventricular-block.

Therefore, it was the purpose of this study to evaluate whether these electrophysiological adaptations could predispose the heart for ventricular tachycardias, specifically for early afterdepolarization-dependent, acquired Torsade de Pointes ${ }^{4-5}$ in this canine model. The cellular basis for the electrophysiologic and structural changes are discussed in a separate article. $^{21}$ Secondly, we were interested to gain more insight in the nature of the volume overload induced hypertrophy by the structural and functional adaptations. 


\section{Methods}

All experiments were performed in accordance with "The Guiding Principles in the Care and Use of Animals" as approved by the American Physiologicall Society (NIH publication $\# 86-23,1996$ ), under the Dutch law on animal experimentation and the European directive for the protection of vertebrate animals used for experimental and scientific purposes, and under the regulations of "The Committee for Experiments on Animals" of the University of Maastricht.

\section{General}

All experiments were performed on adult, anesthetized mongrel dogs of either sex under aseptic conditions with a mean body weight of $25 \pm 4 \mathrm{~kg}$ (range $20-41 \mathrm{~kg}$ ). For the actual experiments, a total of 25 atrioventricular-block dogs were tested and compared to 21 dogs with normal conducted sinus rhythm.

After overnight fasting, anesthesia was induced by 1) premedication i.m. (1 ml $/ 5 \mathrm{~kg}: 10$ mg oxycodon $\mathrm{HCl}, 1 \mathrm{mg}$ acepromazine, and $0.5 \mathrm{mg}$ atropine sulphate) and 2) sodium pentobarbital $(20 \mathrm{mg} / \mathrm{kg}$ i.v. Nembutal, Sanofi). The dogs were artificially ventilated (Dräger, Pulmonat) at a respiratory rate of 12-14 min using a mixture of oxygen, nitrous oxide (40/60\%) and halothane (vapor concentration $0.5-1 \%$ ). Tidal volume was adjusted $(10-25 \mathrm{ml} / \mathrm{kg})$ to maintain the end-expired carbon-dioxide concentration between $3.5-4$ $\%$. A thermal mattress was used to maintain adequate body temperature. During the experiment, the dog received $0.5-110.9 \% \mathrm{NaCl}$ through the cephalic or saphenous vein to prevent volume depletion. This line was also used to administer drugs and to take blood samples.

\section{Induction of chronic, complete atrioventricular-block}

A right sided thoracotomy was performed through the fourth or the fifth intercostal space to: 1) implant an epicardial electrode (Medtronic) at the apex of the left ventricle, and 2) create complete atrioventricular-block by injection of formaldehyde $(37 \%)$ in the atrioventricular-node ${ }^{21}$ Proper care was taken during and after the experiments, including antibiotics (1000 mg ampicillin) and analgesics $(0.015 \mathrm{mg} / \mathrm{kg}$ i.m. buprenorfine, Temgesic, Schering-Plough). Just before closing the chest, the pressure in the lungs was elevated for complete unfolding of this organ.

The majority of the dogs were tested more than once after creation of atrioventricularblock. They were daily checked by the same experienced animal technician to see whether they had appetite for water and food and whether they behaved normally in 


\section{D-SOTALOL}
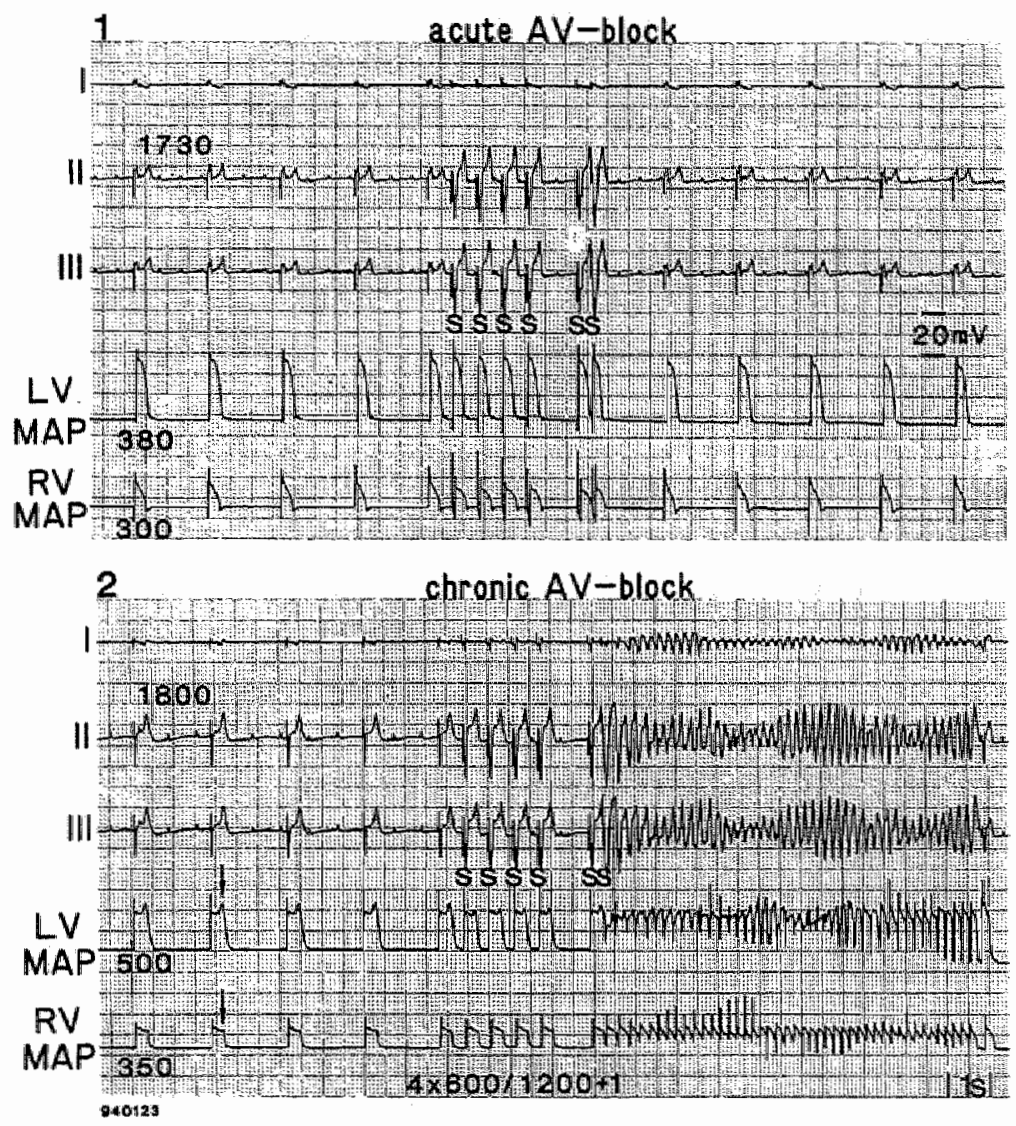

Figure 1: Difference in arrhythmogenic response after $d$-sotalol in acute and chronic atrioventricularblock (AV-block).

This figure consists of three ECG leads $(\mathrm{I}, \mathrm{I}, \mathrm{III})$ and two monophasic action potential (MAP) recordings, one in the right (RV) and one in the left ventricle (LV) at a paper speed of $10 \mathrm{~mm} / \mathrm{s}$. In panel 1 the effect of the different interventions directly after creation of AV-block is shown. After $d$-sotalol, during a paced rhythm of $1730 \mathrm{~ms}$, the duration of the LV action potential (APD) is 380 and the RV APD $300 \mathrm{~ms}$ resulting in a $\triangle A P D$ of $80 \mathrm{~ms}$. Both MAPs are smooth: no early afterdepolarizations (EADs) are present. Performance of a pacing protocol consisting of 4 beats with a cycle length of 600 followed by beat after $1200 \mathrm{~ms}$ and an extra stimulus after $350 \mathrm{~ms}$ does not result in an arrhythmia. Six weeks later (panel 2), at a spontaneous idioventricular cycle length of $1800 \mathrm{~ms}$, administration of $2 \mathrm{mg} / \mathrm{kg} d$-sotalol results in a LV APD of 500 and a RV APD of $350 \mathrm{~ms}$ causing a $\triangle A P D$ of $150 \mathrm{~ms}$. Note that both MAPs now show EADs (arrows). Performance of the same pacing mode results in a self terminating TdP. 
respect to pre-atrioventricular-block situations. Special attention was paid to the presence of signs of heart failure, like dyspnea, ascites, peripheral edema and changes in heart rate.

\section{Pacing}

Electrical stimulation was done from the left epicardial electrode with a custom built programmable stimulator (Maastricht University), that delivers unipolar, rectangular stimuli synchronous to the QRS complexes, with a pulse width of $2 \mathrm{~ms}$ and a stimulus strength of twice the diastolic threshold. As indifferent electrode a needle was placed through the skin.

\section{Serial electrophysiological data and Torsade de Pointes arrhythmias}

A total of 10 animals were instrumented to investigate the response to $d$-sotalol during spontaneous idioventricular rhythm at two time points: acute atrioventricular-block and at 6 weeks of chronic complete atrioventricular-block. At both time points, measurements were performed with a closed thorax. Quadripolar Monophasic Action Potential catheters (steerable Franz ${ }^{\text {TM }}$ Combination Catheter, EP Technologies) were introduced via the carotid artery and the external jugular vein and placed randomly at the endocardium in both ventricles under fluoroscopic guidance. Thereafter the Torsade de Pointes induction protocol was performed during a stable idioventricular rhythm (see further).

\section{Torsade de Pointes induction protocol}

A detailed description of the Torsade de Pointes induction protocol is described elsewhere. ${ }^{4}$ In short, anesthetized animals received two defibrillation patches that were attached to both sides of the chest and connected with a defibrillator. Two different pacing modes were used 1) short long short sequence, 2) An $8+1$ protocol with an interstimulus interval of $600 \mathrm{~ms}$ followed by one extrastimulus. ${ }^{4}$ During both pacing modes the coupling interval of the extrastimulus was shortened from 500 to $300 \mathrm{~ms}$ in $50 \mathrm{~ms}$ steps. After completing the pacing protocol, $d$-sotalol ( $2 \mathrm{mg} / \mathrm{kg} / 5 \mathrm{~min} \mathrm{i} . \mathrm{v}$ ) was administered. The pacing protocol was resumed 10 minutes after the start of the drug injection. A Torsade de Pointes was defined as a polymorphic ventricular tachycardia consisting of $\geq 5$ beats, which twisted around the baseline and occurring in the presence of a prolonged QT(U) duration. A dog was called inducible when Torsade de Pointes could be induced $\geq 3$ times with the same pacing mode. When Torsade de Pointes did not terminate spontaneously within 10 seconds, or when it deteriorated into ventricular fibrillation, cardioversion (60-70 J) was performed. 
Because pacing is necessary to induce Torsade de Pointes, we paid careful attention to the development of early afterdepolarizations and ectopic beats within the pacing mode in those dogs tested twice.

Variables of ventricular repolarization are heart rate dependent and it was previously reported that inducible dogs have a longer cycle length of the idioventricular rhythm than the non-inducible ones. ${ }^{14}$ Therefore, in those dogs studied twice $(n=5)$, we performed the Torsade de Pointes induction protocol after $d$-sotalol at two basic cycle lengths: 1 ) during spontaneous idioventricular rhythm and 2) at a paced cycle length identical to the cycle length at acute atrioventricular-block. When necessary continuous pacing was started 20 minutes after $d$-sotalol.

\section{Monophasic action potentials}

Monophasic action potentials were recorded to observe the occurrence of early afterdepolarizations and to measure the duration of the action potential of the left and right ventricle at $100 \%$ of repolarization. The monophasic action potential signals were amplified with a customized isolated DC-coupled differential amplifier at a frequency range $(0.04-500 \mathrm{~Hz})$ with a $20 \mathrm{mV}$ calibration pulse. The offset of this amplifier is variable and can be adjusted to the recorded signal. Monophasic action potential phases were defined according to the definitions used for transmembrane potentials. Amplitude was defined as the voltage difference between phase 4 and 2 of the signal. Besides a minimal monophasic action potential amplitude of $15 \mathrm{mV}$, a stable configuration and a smooth shape had to be present under control circumstances. Early afterdepolarizations were defined as an interruption of the smooth contour of phase 2 and/or 3 of the action potential, and were examined in both monophasic action potentials.

\section{Serial left ventricular hemodynamic measurements}

All measurements were performed with a closed thorax. For determination of the left ventricular functional status, a catheterization was performed in 6 dogs at 2 time intervals (sinus rhythm/acute atrioventricular-block and 6 weeks after atrioventricular-block) through the carotid artery. Using a solid state micro-manometer transducer catheter (Sentron, Roden, The Netherlands) left ventricular end-systolic pressure, left ventricular end-diastolic pressure and positive left ventricular rate of pressure rise $(+\mathrm{LV} \mathrm{dP} / \mathrm{dt})$ were measured during a fixed paced rhythm of $600 \mathrm{~ms}$ as a mean of 5 beats. In addition, postextrasystolic potentiation (absolute and relative increase in $+\mathrm{LV} \mathrm{dP} / \mathrm{dt}$ ) was determined at these two time points by applying an extra-stimulus from $550 \mathrm{~ms}$ down to 250 
$\mathrm{ms}$ and vice versa during $600 \mathrm{~ms}$ fixed pacing. The recovery interval after the extrastimulus was set on $600 \mathrm{~ms}^{22}$ This protocol was performed before and after a bolus of $20 \mu \mathrm{g} / \mathrm{kg}$ ouabain infused in 1 minute.

In three additional dogs, a conductance catheter (7.5F, Webster Labs) was placed in the apex of the left ventricle directly after creation of atrioventricular-block through the carotid artery. The conductance catheter was connected to a Leycom Sigma-5DF signal conditioner processor (CardioDynamics, Zoetermeer, the Netherlands) to measure left ventricular volume. The correct position of the catheter was assumed if the signals of at least the 4 most distal segments displayed a typical left ventricular volume tracing. ${ }^{23}$ Conductance catheter stroke volume was calibrated by thermodilution stroke volume (Swan Ganz through the jugular vein). Pressure-volume loops were determined at two paced cycle lengths: $600 \mathrm{~ms}$ (comparable to cycle length sinus thythm) and $1200 \mathrm{~ms}$ (comparable to idioventricular rhythm). At the completion of the all procedures, catheters were removed and the vessels were sutured.

\section{Data acquisition and analysis}

All the signals consisting of the six surface ECG leads and either 2 monophasic action potential signals or the pressure signal were simultaneously registered and stored on hard disc during the experiment. All signals were sampled with $1 \mathrm{kHz}$. Applying a custom made computer program (ECG View; Maastricht University) with a resolution of $2 \mathrm{~ms}$ and adjustable gain and time scale, the following parameters were measured: cycle length of the idioventricular rhythm, QT time (lead II), left and right ventricular action potential duration at $100 \%$ repolarization at baseline and at 10 minutes after $d$-sotalol, left ventricular end-systolic pressure, left ventricular end-diastolic pressure and $+\mathrm{LV} \mathrm{dP} / \mathrm{dt}$. All the electrophysiological data reported are the mean of 5 consecutive beats. Interventricular dispersion $(\triangle \mathrm{APD})$ was defined as the difference between the left and right ventricular action potential duration at a certain time point.

\section{Serial plasma neurohumoral data}

Venous blood samples for neurohumoral evaluation were taken while the dogs $(n=7)$ were fully anesthetized in stable hemodynamic conditions (stable Capnograph for 30 minutes). Before the atrioventricular-block operation, two control measurements were taken. Thereafter, samples were collected at $1,2,4,6$, and 8 weeks of chronic, complete atrioventricular-block. 
Fifteen $\mathrm{ml}$ of blood was taken and distributed over 4 pre-chilled tubes containing EDTA, EDTAplus (with $3.6 \mu \mathrm{Mol}$ enalaprilaat), GH (250 IE heparin and glutation), and TE (EDTA and trasylol $50 \mathrm{KIU} / \mathrm{ml}$ blood). The blood samples were immediately centrifuged (15 min, $3400 \mathrm{rpm}, 4^{\circ} \mathrm{C}$ ) and the plasma was decanted into separate tubes, frozen in a dry ice-ethyl alcohol bath and stored at $-80^{\circ} \mathrm{C}$ until the time of assay. Angiotensin II was determined by radio immunoassay following Phenyl column extraction (Amersham Int., Amersham, UK). ${ }^{24}$ Atrial natriuretic factor was determined by RIA (Nichols Institute Diagnostics, Wijchen, The Netherlands) following Sep Pak C18 column extraction. ${ }^{25}$ Aldosteron was assayed by means of a solid-phase protein binding RIA (Diagnostic Products Corporation, LA, USA). ${ }^{26}$ Norepinephrine was assessed by a sensitive fluorimetric method, in which catecholamines are concentrated from plasma by liquidliquid extraction and derivatized with a selective fluorescent agent prior to high performance liquid chromatography. ${ }^{27}$ Most plasma assays were performed in duplicate.

\section{Group comparison of the structural changes}

To assess the amount of hypertrophy due to chronic, complete atrioventricular-block, we determined heart size in two groups. In the first analysis, total heart weight, left and right ventricular weight was determined in 11 dogs in normal conducted sinus rhythm and in 9 atrioventricular-block dogs ( $20 \pm 11$ weeks). For this purpose, the hearts were excised, rinsed with water and stored in $10 \%$ formaldehyde for at least 2 weeks. For ventricular weight, we isolated the ventricles from the atria and we removed the right ventricle, taking the septum as part of the left ventricle. The measurements were corrected for differences in body weight.

In a second group of animals ( 6 dogs in sinus rhythm and 6 dogs after $10 \pm 2$ weeks of atrioventricular-block), the total heart weight was also determined, but now followed by the isolation of 6 transmural specimens from 3 left ventricle (High, Mid and Apical free wall), one septal, and 2 right ventricular (Outflow and Mid free wall) sites. These specimens were formalin fixed and embedded in paraffin. Six mm paraffin sections were stained with Sirius Red F3BA. Morphometric determinations of the collagen volume fraction in all samples (10-15 fields per section) was obtained by one investigator, while two independent observers partly checked the measurements. ${ }^{28}$

To determine the capillary to fiber ratio, 2 transmural tissue samples from right and left ventricle were taken from a $5 \mathrm{~mm}$ thick coronal section at the equator of the heart. The left ventricular tissue sample was taken in between the papillary muscles and the right ventricular tissue sample in the center of this ventricle. The samples were dehydrated in 
ethanol: $2 \times 10 \mathrm{~min}$ in $70 \%, 2 \times 10 \mathrm{~min}$ in $96 \%$ and $2 \times 15 \mathrm{~min}$ in $100 \%$ ethanol. The samples were embedded in Kulzer Technovit 8100 plastic, according to manufacturers guidelines. Cardiomyocyte and capillary basement membranes were stained on $2 \mathrm{~mm}$ thick plastic sections by the Jones silver methamine method. Capillary to fiber ratio was determined on 12 randomly taken fields in one section. Total number of cardiomyocytes and total number of capillaries were counted using a 10 by 10 grid with border correction at a magnification of $400 x^{29,30}$

\section{Group comparison of ventricular ANF mRNA levels}

Total RNA was isolated from cardiac biopsies with TRIzol reagent (Life Technologies). The biopsies were taken from the right and left ventricle of 4 atrioventricular-block dogs ( $9.5 \pm 3$ weeks), 4 dogs in sinus rhythm, and one atrioventricular-block dog with signs of biventricular heart failure. A biopsy of the left ventricle of one dog with aortic stenosis was also included. Samples of canine atrial tissue and hypertrophied rat ventricular tissue served as positive controls. Ten micrograms of RNA were size-fractionated on a denaturating gel ( $1 \%$ agarose, $6.6 \%$ formaldehyde, $1 \times$ MOPS) with $1 \times$ MOPS running buffer. ${ }^{31}$ Thereafter, the RNA was transferred to a nylon membrane (Hybond-N, Amersham) with $10 \times \mathrm{SSC}(1.5 \mathrm{M} \mathrm{NaCl}, 0.15 \mathrm{M} \mathrm{Na-citrate,} \mathrm{pH} 7.0)$ overnight by capillary transfer, and fixed to the filter by heating at $80^{\circ} \mathrm{C}$ for $10 \mathrm{~min}$ followed by crosslinking under UV-light $\left(0.3 \mathrm{~J} / \mathrm{cm}^{2}\right)$. In order to check the quality of the RNA and to correct for possible differences in transfer and loading the filter was stained with $0.04 \%$ methylene blue in $0.5 \mathrm{M}$ acetic acid ( $\mathrm{pH} \mathrm{5.2)}$ ) for $7 \mathrm{~min}$ and then destained in diethyl pyrocarbonate-treated milli-Q water until the ribosomal bands were clearly visible against a white background. A 400 bp fragment of the rat ANF cDNA was labeled with [a-32 P]dCTP (3000 Ci/mmol; DuPont NEN) by random-priming (Radprime, Life Technologies) to a specific activity of $0.5 \times 109 \mathrm{cpm} / \mathrm{mg}$ DNA. ${ }^{32}$ Filters were prehybridized for 2 hours at $58^{\circ} \mathrm{C}$ and hybridized (overnight) at $58^{\circ} \mathrm{C}$ in $6 \mathrm{x}$ SSC containing $0.1 \%$ Ficoll, $0.1 \%$ polyvinylpyrrolidine and $0.1 \%$ bovine serum albumin, $0.5 \%$ SDS and $100 \mathrm{mg} / \mathrm{ml}$ heat denatured salmon sperm DNA. Following overnight hybridization the blots were washed at the appropriate stringency to remove non-specific binding. Afterwards the blots were exposed to Hyperfilm MP (Amersham) with intensifying screens for 2 days at $-80^{\circ} \mathrm{C}$.

\section{Statistics}

Paired and unpaired Student's $t$-test, were applied to compare data obtained in pre-drug condition and after $d$-sotalol or ouabain and between 0 and 5-6 weeks of atrioventricu- 
lar-block. The Chi-square test was used when the data were presented as a proportion, while repeated measures ANOVA was used to determine statistical difference for the post-extrasystolic potentiation and the neurohumoral data. P values $<0.05$ were considered significant. All data are presented as mean \pm standard deviation (SD), unless otherwise stated.

Table 1: Electrophysiological effects of chronic complete atrioventricular-block and effect of $d$-sotalol

\begin{tabular}{|c|c|c|c|c|c|c|}
\hline & \multicolumn{2}{|c|}{ baseline } & \multicolumn{4}{|c|}{$d$-sotalol } \\
\hline & 0 wks & 5 wks & 0 wks & & 5 wks & \\
\hline CL-IVR (ms) & $1600 \pm 280$ & $1615 \pm 280$ & $1720 \pm 465$ & & $1935 \pm 425$ & \\
\hline $\mathrm{QT}(\mathrm{ms})$ & $315 \pm 25$ & $390 \pm 65^{*}$ & $385 \pm 40 \dagger$ & $+22 \%$ & $485 \pm 85^{*} *$ & $+25 \%$ \\
\hline LV APD (ms) & $295 \pm 20$ & $390 \pm 60^{*}$ & $340 \pm 40 \dagger$ & $+15 \%$ & $495 \pm 95+*$ & $+28 \%$ \\
\hline RV APD (ms) & $260 \pm 20$ & $315 \pm 40^{*}$ & $295 \pm 20 \dagger$ & $+15 \%$ & $370 \pm 55 \dagger^{*}$ & $+18 \%$ \\
\hline$\triangle \mathrm{APD}(\mathrm{ms})$ & $40 \pm 35$ & $70 \pm 30$ & $45 \pm 30 \dagger$ & $+12 \%$ & $125 \pm 65 t^{*}$ & $+80 \%$ \\
\hline EADs & $0 / 14$ & $1 / 18$ & $4 / 14$ & & $9 / 18 \dagger$ & \\
\hline $\mathrm{TdP}$ & $0 / 9$ & $1 / 10$ & $0 / 9$ & & $6 / 10^{+}$ & \\
\hline
\end{tabular}

Data are presented as mean $\pm S D$ or as ratios. The percent increase after $d$-sotalol is indicated.

wks: weeks, CL-IVR: cycle length of the idioventricular rhythm, LV: left ventricle, RV: right ventricle, APD: action potential duration, EADs: early afterdepolarizations, TdP: Torsade de Pointes, * $\mathrm{p}<0.05$ compared to baseline 0 weeks, $t: p<0.05$ baseline versus $d$-sotalol

\section{Results}

\section{Electrophysiological adaptations and induction of acquired Torsade de Pointes arrhythmias after chronic, complete atrioventricular-block}

During creation of atrioventricular-block, we lost one dog due to ventricular fibrillation. In 7 of the remaining 9 acute atrioventricular-block dogs, the criteria with respect to the quality of both monophasic action potentials were met to ensure appropriate data sampling ( $7 \times 2=14$ monophasic action potentials, table 1$)$.

In between experiments, one of these 7 dogs died. In another dog we could not record adequate monophasic action potential signals during the second experiment, i.e. five dogs could be fully analyzed at both time points. To increase the number of dogs with chronic 


\section{ACUTE COMPLETE AV-BLOCK}

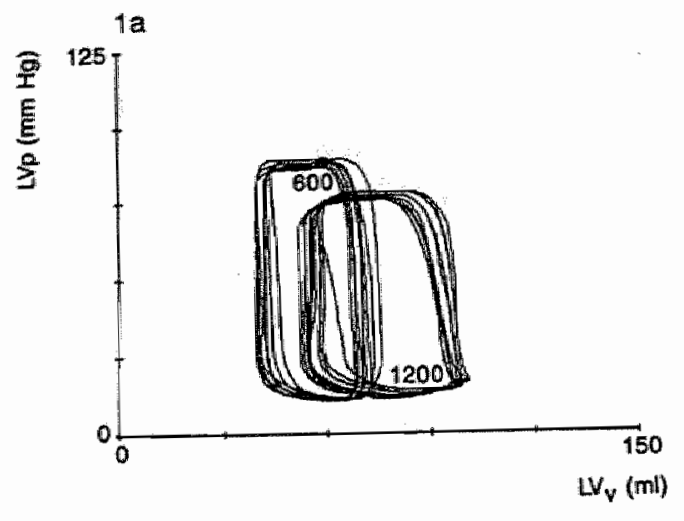

\begin{tabular}{lcc}
\hline & \multicolumn{2}{c}{ Acute AV-block } \\
\cline { 2 - 3 } Paced CL (ms) & 600 & 1200 \\
\hline ESP (mm Hg) & 88 & 75 \\
EDP (mm Hg) & 11 & 16 \\
SV (mi) & 32 & 41 \\
CO (l/min) & 3.2 & 2.1 \\
EDV (mi) & 70 & 99 \\
ESV (ml) & 38 & 58 \\
pos LV dP/dt (mm Hg/s) & 1022 & 669 \\
neg LV dP/dt (mm Hg/s) & -717 & 498 \\
tau (ms) & 58 & 71 \\
PFR (mis) & 305 & 295 \\
PER (mis) & 188 & 192 \\
\hline
\end{tabular}

Figure 2: This figure illustrates the hemodynamic changes observed after atrioventricular-block (AVblock) using 2 heart frequencies: continuous paced (CP) from an left ventricular epicardial electrode at $600 \mathrm{~ms}$ (previous sinus rate) and at $1200 \mathrm{~ms}$ (AV-block rhythm) after a stabilization period of 20 minutes. Hemodynamic data are gathered in the table. Doubling of the paced cycle length leads to: 1) increase in stroke volume (SV), 2) decrease in cardiac output (CO) and diastolic volume (EDV), 3) an increase in end-diastolic pressure and volume (EDP and EDV), 4) a decrease in end-systolic pressure and an increase in volume (ESP and ESV), and 5) a right and downward shift of the pressure-volume loop with an increase in the area of the loop, indicating volume overload.

atrioventricular-block, we added 2 dogs that were only tested at 6 weeks of atrioventricular-block. At chronic atrioventricular-block 10 dogs were given $d$-sotalol, of which 9 had adequate monophasic action potentials for electrophysiological analysis $(9 \times 2=18$ monophasic action potentials, table 1). The data will be presented as a group comparison and as a serial comparison for the 5 dogs tested twice with adequate monophasic action potential signalls. 


\section{Group comparison}

After creation of complete atrioventricular-block, a stable idioventricular rhythm evolved with a cycle length of $\pm 1600 \mathrm{~ms}$, which was maintained over time (table 1). At baseline all dogs had smooth monophasic action potential signals ( $n=7$ ); no early afterdepolarizations $(0 / 14)$ and no Torsade de Pointes induction by pacing $(0 / 9$, table 1 in the left column).

Five weeks of chronic atrioventricular-block led to a significant increase in all repolarization parameters (table 1, second column). The $\triangle A P D$ augmented from $40 \pm 35$ to $70 \pm 30$ $\mathrm{ms}$ (non-significant), due to the absolutely and relatively larger increase of the left ventricular action potential duration compared with the right ventricular action potential duration. At chronic atrioventricular-block, one dog had very pronounced repolarization disorders: QTU waves on ECG, early afterdepolarizations in the left ventricle (1/18, table 1), and a large interventricular $\triangle A P D(130 \mathrm{~ms})$. Torsade de Pointes could be induced in this animal $(1 / 10$, table 1$)$.

\section{Effect of d-sotalol}

In half of the experiments directly following atrioventricular-block period, $d$-sotalol increased the cycle of the idioventricular rhythm to such an extent that hemodynamics became compromized (fall in capnograph $\mathrm{CO}_{2}$-value) making pacing at a faster rhythm necessary. We chose to pace at the pre- $d$-sotalol idioventricular rhythm value. Therefore, the values shown can underestimate the slowing of the idioventricular rhythm by $d$-sotalol.

At a comparable cycle length of the idioventricular rhythm, $d$-sotalol increased the QT time and action potential duration in both ventricles (table 1, third column). Action potential durations were prolonged less in comparison to the effect of the electrical remodeling process after atrioventricular-block. The $d$-sotalol induced prolongation occurred uniformly so that no effect on interventricular $\triangle A P D$ was seen. Early afterdepolarizations were visible in $4 / 14$ monophasic action potentials, but no Torsade de Pointes could be induced by pacing $(0 / 9$, table 1$)$.

In the dogs with chronic complete atrioventricular-block the effect of $d$-sotalol on left ventricular action potential duration was much more pronounced than in the dogs with acute complete atrioventricular-block ( $+28 \%$ versus $+15 \%, \mathrm{p}<0.05$, table 1 in the fourth column). Early afterdepolarizations also developed more frequently after $d$-sotalol during chronic atrioventricular-block $(9 / 18, \mathrm{p}<0.05)$. All these changes led to an increased $\triangle A P D$ of $125 \pm 65 \mathrm{~ms}$ and to the induction of Torsade de Pointes in $6 / 10$ animals. 
Table 2: Incidence of early afterdepolarizations (EAD), amount of dispersion of repolarization ( $\triangle A P D)$ and incidence of Torsade de Pointes arthythmias (TdP) at 0 and 5 weeks after $d$ - sotalol in the same dog

\begin{tabular}{|c|c|c|c|c|c|c|c|}
\hline Dog & LV/RY & EAD 0 & $\triangle A P D$ & TdP 0 & EAD 5 & $\triangle \mathbf{A P D}$ & TdP 5 \\
\hline \multirow[t]{2}{*}{1} & LV & + & 75 & - & + & 180 & + \\
\hline & $\mathrm{RV}$ & $t$ & & & + & & \\
\hline \multirow[t]{2}{*}{2} & $L V$ & - & 35 & - & - & 70 & - \\
\hline & RV & . & & & - & & \\
\hline \multirow[t]{2}{*}{3} & $\mathrm{LV}$ & - & 80 & - & + & 150 & + \\
\hline & RV & - & & & + & & \\
\hline \multirow[t]{2}{*}{4} & LV & - & 25 & - & + & 80 & + \\
\hline & RV & - & & & + & & \\
\hline \multirow[t]{2}{*}{5} & LV & + & 40 & - & + & 125 & + \\
\hline & RV & + & & & + & & \\
\hline Total & & $4 / 10$ & $50 \pm 25$ & $0 / 5$ & $8 / 10$ & $120 \pm 45^{*}$ & $4 / 5$ \\
\hline
\end{tabular}

Data are presented as individual data, as mean $\pm \mathrm{SD}(\mathrm{ms})$ or as ratios, *: $\mathrm{P}<0.01,0$ versus 5 weeks $\mathrm{LV}$ : left ventricle, RV: right ventricle, APD: action potential duration, $\triangle \mathrm{APD}$ : $\mathrm{LV}$ APD-RV APD, $0=$ acute ( 0 weeks) and $5=$ chronic atrioventricular-block ( $>5$ weeks)

\section{Serial testing}

When using the dog as its own control, similar results were obtained (table 2). At the time of acute atrioventricular-block, the combination of pacing and $d$-sotalol never resulted in Torsade de Pointes or ectopic beats (figure 1, panel 1). In the chronic phase, this combination resulted in Torsade de Pointes induction in the majority of the dogs (4/5, figure 1 , panel 2). Torsade de Pointes induction was associated with a longer action potential duration, an increased $\triangle A P D(50 \pm 25$ versus $120 \pm 45 \mathrm{~ms}, \mathrm{p}<0.05)$ and more frequent development of early afterdepolarizations after $d$-sotalol ( $4 / 10$ versus $8 / 10$, table 2). The non inducible dog had the smallest $\triangle A P D$ and no early afterdepolarizations. Programmed electrical stimulation was repeatedly interrupted by ectopic beats in dogs with chronic atrioventricular-block while at acute atrioventricular-block this was never the case. After correcting for the somewhat slower heart rate observed in these (inducible) 
$1 \quad$ Absolute LV PESP response $(\mathrm{n}=6)$

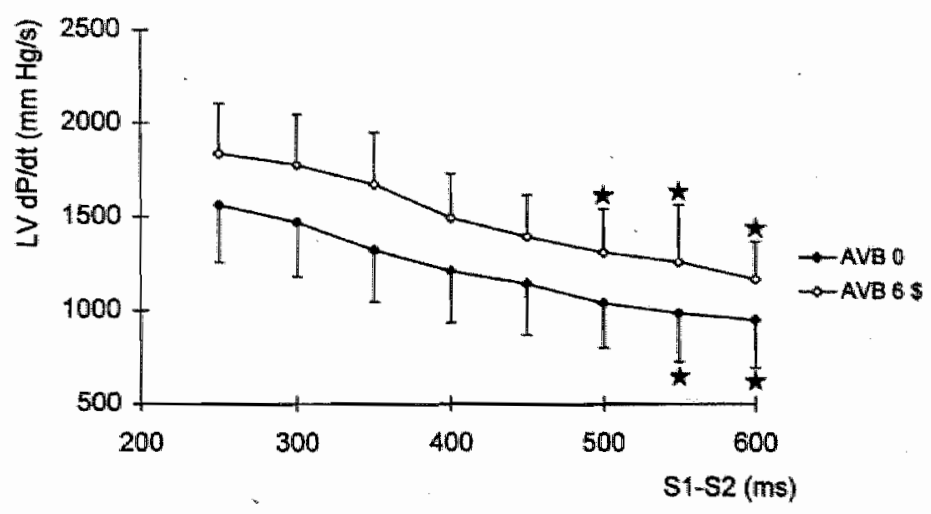

2 Relative LV PESP response ( $\mathrm{n}=6$ )

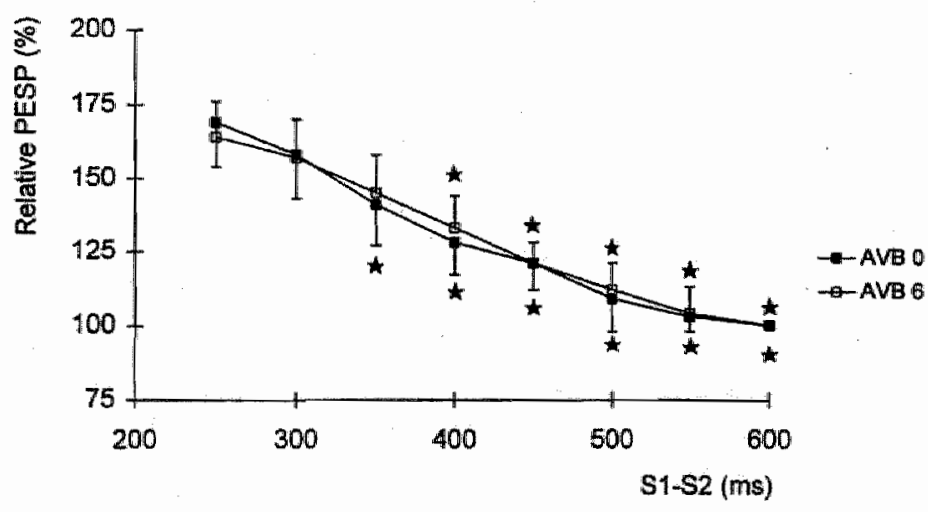

Mean $\pm S D, \star p<0.05$ versus $S 1-S 2=250 \mathrm{~ms}, \$: p<0.05$ versus AVB 0

Figure 3: Post Extra Systolic Potentiation (PESP) at acute and chronic atrioventricular-block (AVB). In panel 1, the absolute left ventricular (LV) PESP response ( $+\mathrm{LV} d P / d t$ on the $y$-axis) is shown when the coupling interval of the extra-stimulus is decreased from $600 \mathrm{~ms}$ (steady state) to $250 \mathrm{~ms}$ ( $\mathrm{x}$-axis). The curve at chronic atrioventricular-block is significantly higher than at acute atrioventricular-block, while the potentiation is present at both times: the value at $250 \mathrm{~ms}$ is significantly higher $\left({ }^{*}\right)$.

In panel 2, these curves are generated using the relative increase in $+\mathrm{LV} d \mathrm{dP} / \mathrm{dt}$, related to steady state 600 ms values. Again shortening of the extra-stimulus interval leads to more potentiation (*). However, the curves are now identical. 


\section{Chaprer 5}

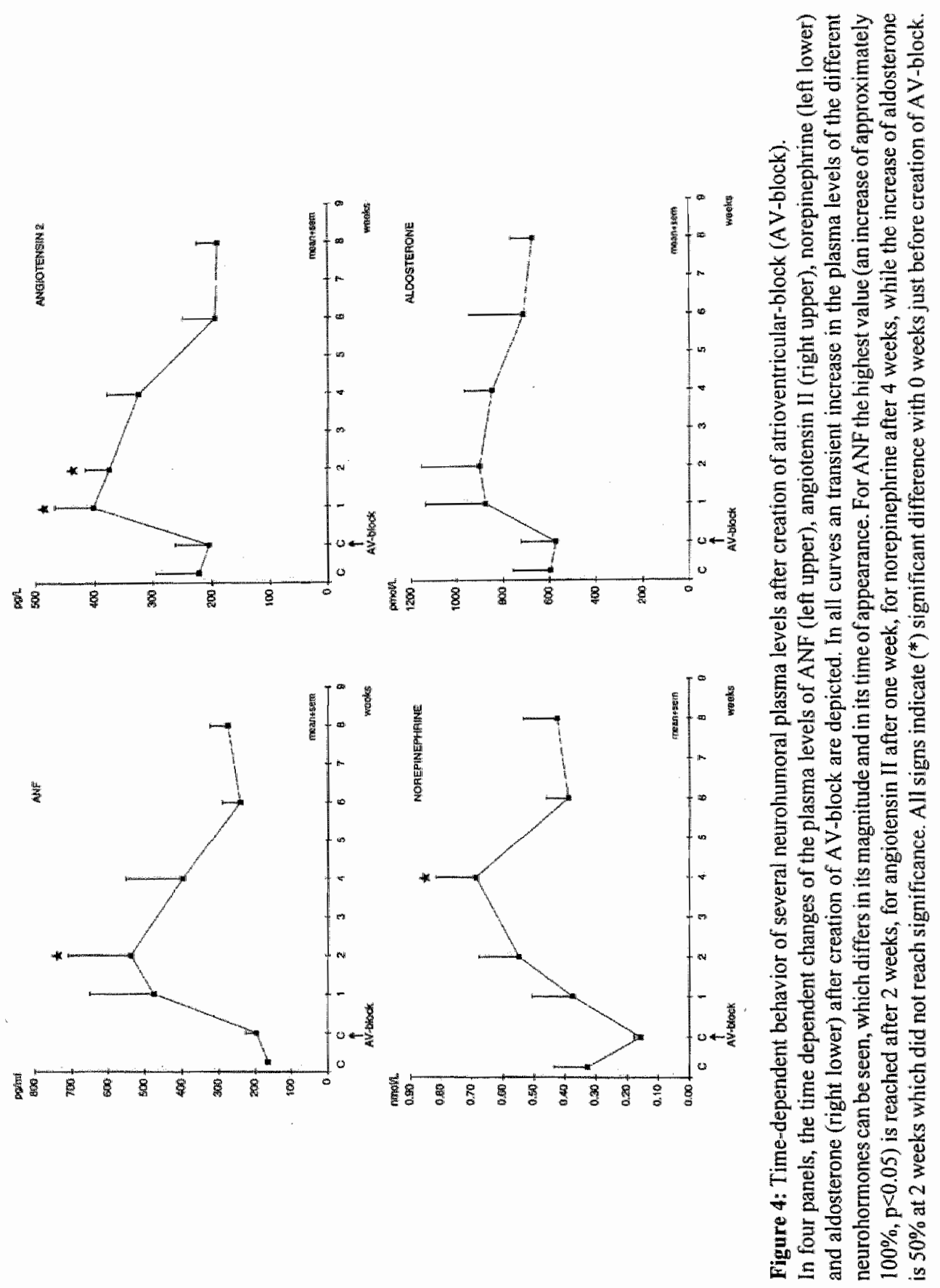


dogs after chronic atrioventricular-block, pacing did not lead to a different response: they remained inducible.

\section{Hemodynamic evaluation of LV function}

\section{Acute state}

Under complete (halothane) anesthesia, the cycle length of the sinus rhythm was $540 \pm 50$ $\mathrm{ms}$. Creation of atrioventricular-block resulted in these dogs in an idioventricular rhythm with a cycle length of $1320 \pm 280 \mathrm{~ms}(p<0.05)$. This decrease in heart rate was accompanied with an increase in left ventricular end-diastolic pressure from $9 \pm 4 \mathrm{~mm} \mathrm{Hg}$ to $16 \pm 4$ $\mathrm{mm} \mathrm{Hg}(\mathrm{p}<0.05)$, while left ventricular end-systolic pressure remained similar $(87 \pm 18$ $\mathrm{mm} \mathrm{Hg}$ during sinus rhythm versus $91 \pm 16 \mathrm{~mm} \mathrm{Hg}$ ). An example of a pressure-volumeloop illustrating bradycardia induced volume overload is shown in figure 2.

\section{Chronic state}

Under anesthesia at a constant paced cycle length of $600 \mathrm{~ms}$, the left ventricular end-systolic pressure was $88 \pm 9 \mathrm{~mm} \mathrm{Hg}$, the end-diastolic pressure $11 \pm 3 \mathrm{~mm} \mathrm{Hg}$ and the $+\mathrm{LV} \mathrm{dP} / \mathrm{dt}$ was $941 \pm 242 \mathrm{~mm} \mathrm{Hg} / \mathrm{s}$ directly after creation of atrioventricular-block. There were no differences in hemodynamic status of the left ventricle after 6 weeks of atrioventricular-block: $93 \pm+5 \mathrm{~mm} \mathrm{Hg}, 8 \pm 4 \mathrm{~mm} \mathrm{Hg}$, and $1145 \pm 212 \mathrm{~mm} \mathrm{Hg} / \mathrm{s}$ respectively. In figure 3 (panel 1), the post-extrasystolic potentiation results are shown for the two time points. The absolute $+\mathrm{LV} \mathrm{dP/dt}$ was a little higher during steady state pacing at 6 weeks of atrioventricular-block $(p<0.05)$. This difference was maintained with the shortening of the extra-stimulus. At both time points a clear increase in post pacing potentiation ( $+\mathrm{LV} \mathrm{dP} / \mathrm{dt}$ ) is visible on shortening of the extra-stimulus $(\mathrm{p}<0.05)$. When post-extrasystolic potentiation was relatively expressed, no difference between the two groups could be detected (figure 3, panel 2). A similar response was also seen after administration of ouabain in a therapeutic dose of $20 \mu \mathrm{g} / \mathrm{kg} / \mathrm{min}$, that increased left ventricular end-systolic pressure with $\pm 6 \%$ (data not shown).

\section{Neurohumoral evaluation}

At two weeks, but not after 6 weeks atrioventricular-block ANF ( $200 \pm 30$ versus $540 \pm 170$ $\mathrm{pg} / \mathrm{ml})$ and norepinephrine were significantly $(\mathrm{p}<0.05)$ elevated compared to control (Figure 4, left panels). Also plasma angiotensin II ( $205 \pm 55$ versus $405 \pm 65 \mathrm{pg} / \mathrm{l}$ ) was transiently increased, while aldosterone did not show a significant increase $(595 \pm 160$ versus $900 \pm 250 \mathrm{pmol} / 1$, figure 4 , right panels). 

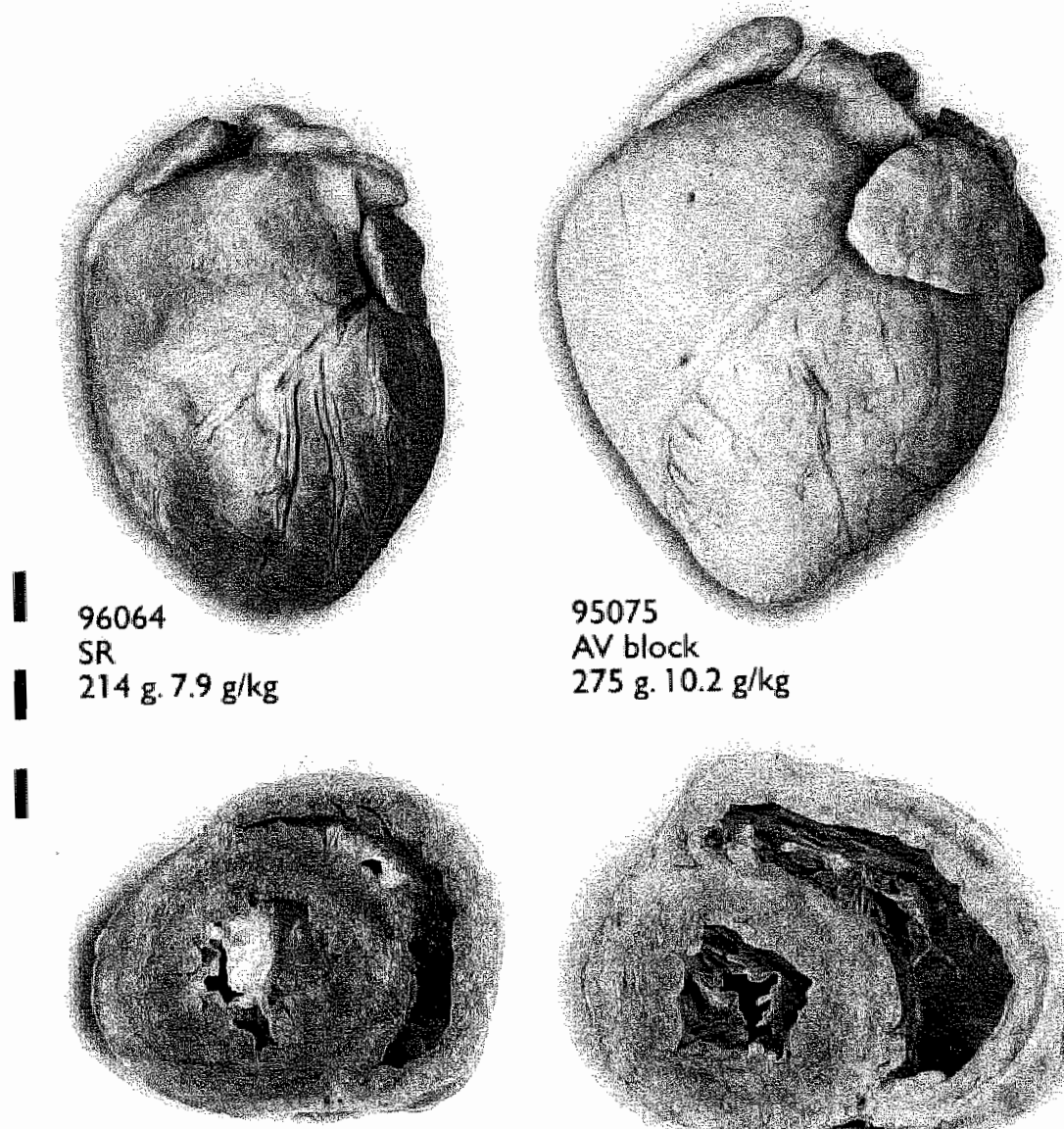

94087

$S R$

$195 \mathrm{~g} .7 .1 \mathrm{~g} / \mathrm{kg}$

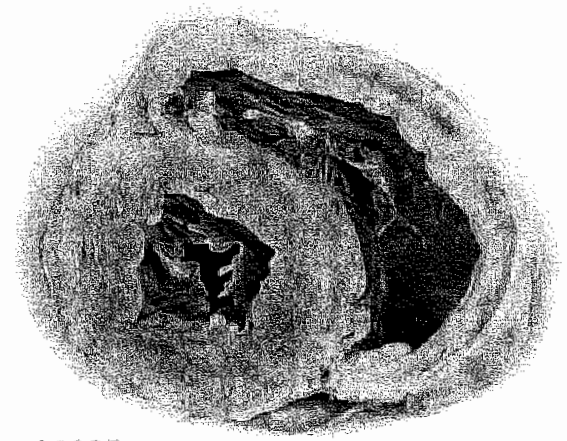

93035

AV block

$265 \mathrm{~g} .10 .1 \mathrm{~g} / \mathrm{kg}$

Figure 5: Biventricular hypertrophy after atrioventricular-block (AV-block).

In the upper part, a frontal view of the heart of two animals with comparable body weight is shown. It is clear that the size of the atrioventricular-block dog heart (right part) is larger and heavier (10.2 g/kg versus $7.9 \mathrm{~g} / \mathrm{kg}$ ) than that of a sinus rhythm dog (left part)

Similar observations can be seen using a transversal view, in which the marked increase in right ventricular size (both cavity and wall thickness) can be appreciated. The left ventricle showed eccentric hypertrophy: predominantly an increase in length of the cavity with similar values of wall thickness. 

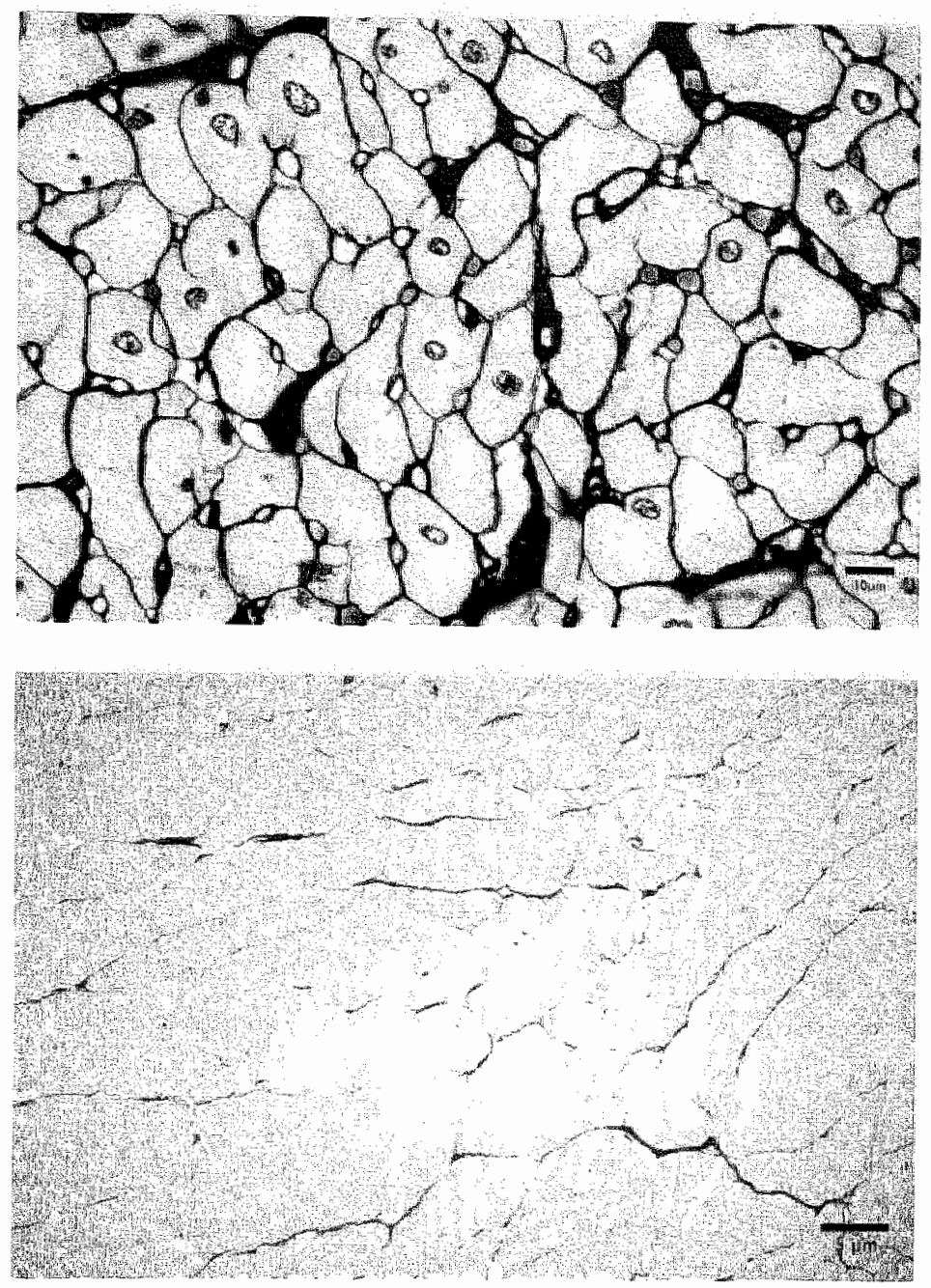

Figure 6: Structural changes.

In the upper panel, a representative photomicrograph of a Jones silver stained plastic section is shown for a sinus rhythm dog (93011). Single cardiomyocytes are indiwidually covered by a silver stained basement membrane. Capillaries are visualized by silver stained basement membranes. These capillaries surround the cardiomyocytes. Occasionally erythrocytes are trapped in the lumen of the capillaries (pink anuclear cells). Scale bar is $10 \mu \mathrm{m}$. In the lower part, a photomicrographic of a Sirius Red stained paraffin section is illustrated for a atrioventricular-block dog (94017). Sirius Red stains fibrillar collagen fibrils red. Note: no replacement fibrosis and no thickening of intercellular collagen fibrils. Scale bar is $4 \mu \mathrm{m}$. 


\section{Heart weights}

Assessment of the heart weight in dogs with chronic atrioventricular-block ( $20 \pm 11$ weeks) revealed a significant increase in mass when compared to the hearts of dogs in sinus thythm with comparable body weight (table 3). This increase is present in the left and right ventricle (table 2), resulting in biventricular hypertrophy. In figure 5 , the heart of a sinus rhythm dog is compared to an atrioventricular-block dog of equal body weight and size (upper panel). The nature of the left ventricular hypertrophy seemed eccentric: the size of the cavity increased while the wall thickness of the septum and the left ventricular free remained similar or somewhat increased (figure 5, lower panel). At the basal part of the right ventricle a clear bulging developed. The size of this cavity increased considerably which seemed also to go along with thickening of the right ventricular free wall. However, no definitive statements concerning volumes and wall thickness can be made using hearts which were not fixed at a given filling pressure or volume.

\section{Collagen and capillary to fiber ratio}

The (corrected) heart weights of the atrioventricular-block dogs used to determine histological changes (table 4), were similarly increased compared to sinus rhythm dogs as the ones reported in table 3 . In the sinus rhythm dogs all capillary to fiber ratios were well over 1. A representative example of a Jones silver methamine staining is shown in the upper part of figure 6 , confirming the literature. ${ }^{30}$ The capillary to fiber ratio of dogs with atrioventricular block was smaller in 2 of the 3 comparisons: for total ventricle and for the left ventricle. But still, the ratios presented for the atrioventricular-block dogs were well over 1 .

Collagen volume fraction did not differ between the two groups, either presented as total ventricular volume or specified to either ventricle. An example of the Sirius Red staining is shown in the lower part of figure 6.

\section{Ventricular expression of ANF mRNA}

In figure 7, the Northern blot is presented for the expression of ANF. As expected, ANF mRNA expression can be identified in the hypertrophied left ventricle of the rat and the right atrium of the dog (control samples, figure 7, right panel). Also in accordance with the literature is the absence of expression of ANF mRNA in the sinus rhythm dogs. This study demonstrates that ANF mRNA was not expressed in the left ventricle of atrioventricular-block dogs, nor in the chronic heart failure dog after atrioventricular-block (CHF atrioventricular-block), nor in a dog with aortic stenosis (AS dog). Similar findings were 
obtained for the right ventricle (left panel), with the exception that the atrioventricularblock dog with congestive heart failure did show a low level of expression of ANF mRNA.

Table 3: Heart weight of dogs in sinus rhythm (SR) compared with dogs with chronic complete atrioventricular-block (AV: 20 weeks, range 10-34)

\begin{tabular}{lll}
\hline & SR, N=11 & AV, N=9 \\
\hline Heart weight (g) & $225 \pm 34$ & $280 \pm 64^{*}$ \\
H/BW $(\mathrm{g} / \mathrm{kg})$ & $7.7 \pm 1.2$ & $11.2 \pm 1.9^{*}$ \\
$\mathrm{LV}(\mathrm{g})$ & $125 \pm 26$ & $145 \pm 32$ \\
$\mathrm{RV}(\mathrm{g})$ & $40 \pm 8$ & $65 \pm 22^{*}$ \\
$\mathrm{LV} / \mathrm{BW}(\mathrm{g} / \mathrm{kg})$ & $4.3 \pm 0.9$ & $5.8 \pm 0.9^{*}$ \\
RV/BW $(\mathrm{g} / \mathrm{kg})$ & $1.4 \pm 0.4$ & $2.6 \pm 0.9^{*}$ \\
$\mathrm{BW}(\mathrm{kg})$ & $29.6 \pm 5.6$ & $25.1 \pm 5.6$ \\
\hline
\end{tabular}

H/BW: Heart/body weight $(\mathrm{g} / \mathrm{kg})$, LV: left ventricular weight $(\mathrm{g})$, RV: right ventricular weight (g), LV/BW: LV/body weight (g/kg), RV/BW: RV/body weight.

*: $\mathrm{p}<0.05$ versus sinus thythm, unpaired $t$-test

\section{Discussion}

\section{Adaptive or physiological hypertrophy}

From clinical and experimental studies it is well known that chronic pressure or volume overload of the heart results in myocardial adaptation (cardiac remodeling). Several canine models of ventricular hypertrophy and/or heart failure have been described ${ }^{6-8,33-}$ 42 which differ markedly in their presentation in regard to 1) heart (myocyte) function (compensated or decompensated), 2) level, nature and location of myocardial growth, 3) cardiac stiffness (interstitial fibrosis), 4) energy supply (capillary to fiber ratio), 5) (re)expression of certain genes, and 6) activation of the plasma or tissue renin-angiotensin-aldosterone system. 
In this study, we confirm that bradycardia as a result of chronic, complete atrioventricular-block leads to biventricular hypertrophy. ${ }^{6-8}$ The increase in the weight of the left ventricle is in accordance with the literature of this model ${ }^{7,8}$ and other forms of volume overload. ${ }^{43}$ In models of pressure overload hypertrophy, ${ }^{15,39-41}$ equal or higher values have been reported. The reported increase in the right ventricular weight is substantial when compared with other models of volume ${ }^{43}$ or pressure overload. ${ }^{39}$ In volume overload, myocytes increase primarily in length ${ }^{44}$ which is in accordance with the eccentric hypertrophy noticed by us, although small increases in wall thickness have been described. ${ }^{8}$ In the atrioventricular-block dog, these findings have been confirmed on the cellular level. ${ }^{21}$

\section{Expression of ANF(Northern Blot)}

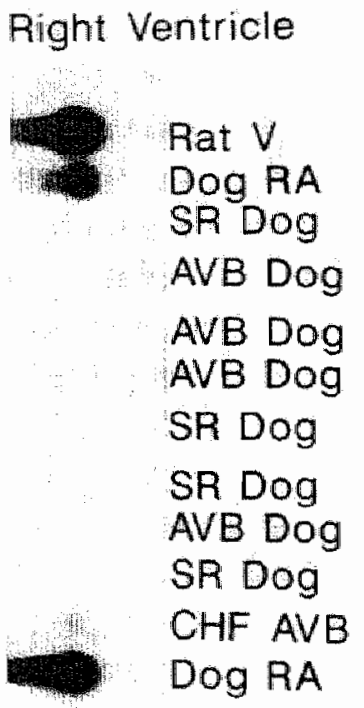

Left Ventricle

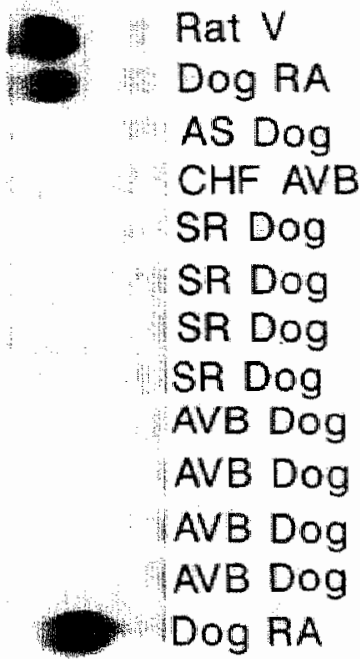

Figure 7" Expression of ANF with Northern Blot

Expression of ANF mRNA is visualized for different tissue obtained in different species. As control, ventricular expression of ANF is shown for the hypertrophied rat heart (rat V). Also expression of ANF can be demonstrated in the dog right atrium $(\operatorname{dog} R A)$. However, in neither of the ventricles of the dogs studied could ANF be detected: not in the right and left ventricle of sinus rhythm dogs (SR dogs), not in the left and right ventricle of normal atriowentricular-block dogs (AVB dogs), not in the left ventricle of a dog with aorta stenosis (AS dog) nor in the left ventricle of an AVB dog with evident congestive heart failure (CHF). Only in the right ventricle of this dog expression of ANF could be seen (left lower part). 
Table 4: Structural changes of the heart after chronic, complete atrioventricular-block

\begin{tabular}{lll}
\hline & SR, $\mathrm{N}=6$ & $\mathrm{AV}, \mathrm{N}=6$ \\
\hline Heart weight $(\mathrm{g})$ & $204 \pm 35$ & $320 \pm 24^{*}$ \\
Body weight $(\mathrm{kg})$ & $26.7 \pm 5.2$ & $26.7 \pm 4.3$ \\
HBW $(\mathrm{g} / \mathrm{kg})$ & $7.7 \pm 0.6$ & $12.2 \pm 1.7^{*}$ \\
Collagen, total $(\%)$ & $2.41 \pm 0.93$ & $1.97 \pm 0.98$ \\
Collagen, LV $(\%)$ & $2.18 \pm 0.84$ & $1.87 \pm 0.80$ \\
Collagen, RV $(\%)$ & $2.87 \pm 0.98$ & $2.16 \pm 1.30$ \\
CF ratio, total & $1.25 \pm 0.15$ & $1.11 \pm 0.10^{*}$ \\
CF ratio, LV & $1.26 \pm 0.08$ & $1.13 \pm 0.09 *$ \\
CF ratio, RV & $1.23 \pm 0.21$ & $1.09 \pm 0.13$ \\
\hline
\end{tabular}

SR: sinus rhythm dogs, AV: atrioventricular-block dogs (10 2 wks), H: heart, BW: body weight, LV: left ventricle, $\mathrm{RV}$ : right ventricle, $\mathrm{CF}$ : Capillary-Fiber, ${ }^{*} \mathrm{p}<0.05$ atrioventricular-block dogs versus sinus rhythm dogs

From the literature, it is unclear to what extent the induced hypertrophy can maintain optimal ventricular function. After an initial decrease, left ventricular performance has been reported not to be depressed at any evaluated state of hypertrophy up to 3 years of atrioventricular-block. $^{6,8}$ Other studies, performed on smaller dogs, reported however the appearance of right and left sided heart failure, either spontaneously or exercise induced. ${ }^{45,46}$ The majority of our dogs did not show any physical sign of decompensation in their daily existence at the slow idioventricular rhythm. Also when measuring pressures under halothane anesthesia, the left ventricle seemed to respond adequately to the increased volume: neither during steady state rhythms of $600 \mathrm{~ms}$, nor during the post-extrasystolic potentiation protocol was there any sign of impaired left ventricular systolic, or diastolic function. This in contrast to the pacing induced heart failure dog in whom impaired cardiac function has been described systematically both at steady state rhythms and during post-extrasystolic potentiation. ${ }^{22,33,35,36}$

Our values of contractility seem to be rather low. We believe that this is due to the applied anesthesic (halothane) regimen. Because this anesthetic regimen was used by us in the 
past when inducing ventricular arrhythmias, ${ }^{3-5}$ we decided not to change this regimen for comparative reasons. Furthermore, we calculated $+\mathrm{LV} d \mathrm{~d} / \mathrm{dt}$ in stead of $\max +\mathrm{LV}$ $\mathrm{dP} / \mathrm{dt}$ due to a limitation in our software programm.

In the different canine models of heart failure, several reports have described huge increases in the plasma levels of neurohumoral parameters (ANF, angiotensin $\mathrm{II}$, renin, catecholamines, and aldosterone) when cardiac failure develops. ${ }^{33-35,39,41,42}$ In time, these values remain elevated or increase further with the progression of the disease, until the situation is reversed or normalized. The return of all the neurohumoral factors to the baseline values in this model (figure 4) indicates normalization of function: compensated hypertrophy.

Expression of ANF mRNA has been suggested to be an early indicator for ventricular decompensation, which increases progressively with development of failure. ${ }^{37}$ Again, the lack of expression of ANF mRNA in the ventricles of the atrioventricular-block dogs indicate absence of heart failure.

There is evidence that pressure overload induced hypertrophy is associated with interstitial fibrosis and deterioration of function while this has not been observed by volume induced hypertrophy. ${ }^{28}$ In canine ventricular myocardium, normal values of the volume content of collagen have been reported to be in the $2.5-4.3 \%$ range ${ }^{28,30}$ In dogs with heart failure, an increased collagen content has been described. ${ }^{28,30}$ This pathogenic state is further characterized by interstitial edema, disnuption of collagen fibers, reduced capillary to fiber ratios and malalignment of muscle fibers. Sabbah et al., ${ }^{30}$ in a study using dogs with heart failure induced by repeated micro-embolization, showed reactive interstial fibrosis in parts of the left ventricle which was associated with a reduced capillary to fiber ratio $(0.92 \pm 0.02)$. In other regions in which no fibrosis was present, normal capillary to fiber ratios around $1.05 \pm 0.03$ were found. In this study, we found ventricular hypertrophy but no evidence for fibrosis nor for a markedly decreased capillary to fiber ratio suggesting a parallel increase in muscle, vessels and connective tissue components.

Information concerning right ventricular function was not obtained in this or other studies. However, based on the above observations (neurohumoral and ANF mRNA data), we believe that also this chamber is well adapted to the increased demand.

Based on 1) the transient increase in the neurohumoral response, which was normalized again at the moment we performed the functional tests for a second time, 2) the inability to detect expression of ANF mRNA in the ventricles, 3) the functional tests and the physical well being of these dogs, and 4) the absence of interstitial fibrosis and a normal 
capillary to fiber ratio, we conclude that the majority of these dogs have compensated, biventricular hypertrophy, which has been referred to as physiological or adaptive hypertrophy.

During these experiments, there was one exception. In this dog not included in the group comparisons, signs of heart failure were clearly seen, aldosterone levels were elevated to above $2000 \mathrm{pmol} / 1$ angiotensin II reached $660 \mathrm{pg} / 1$, fibrosis was present and ANF mRNA expression was seen in the right ventricle (figure 7 ).

\section{Electrical remodeling and increased susceptibility for Torsade de Pointes-arrhythmias}

In contrast to the above described functional, structural and neurohumoral changes, the electrophysiological adaptation has been less well documented, especially in relation to the arrhythmogenic consequences of this remodeling process. ${ }^{13-17}$ The only systematic finding is the prolongation of the duration of the left ventricular action potential, both in vivo (monophasic action potential-recordings or QT-duration) as in vitro. To provide a link between the longer action potentials found in hypertrophied hearts and the arrhythmogenic consequence of this remodeling, we have tested the hypothesis that ellectrical remodeling predisposes the heart for early afterdepolarizations, interventricular dispersion and Torsade de Pointes arrhythmias.

This animal model has been used by us for a long time to study ventricular arrhythmias. Normally, we wait 2 weeks after creation of atrioventricular-block before the experiments are started. After that time point, we 1) have the impression that the animals were able to tolerate the bradycardia well (without the need of a pacemaker, see above) and 2) notice that the ability to induce ventricular tachycardias by different interventions (ouabain or class III drugs) is very reproducible. Ventricular tachycardias are easily induced in the majority of the dogs that have been tested for a maximum period of 3 years, although the majority was kept for 10-20 weeks.

Mild hypertrophy caused by pressure overload has been shown to prolong left ventricular action potential duration by $14 \%$ both in situ, ${ }^{15}$ and in vitro. ${ }^{17}$ The present study shows that chronic atrioventricular-block leads to a prolongation of $L V$ action potential duration with $32 \%$. This different increase in LV action potential duration, despite a similar amount of LV hypertrophy, could be due to differences in cycle length between the two groups: $800 \mathrm{~ms}$ in the pressure ${ }^{15}$ versus $1600 \mathrm{~ms}$ in our dogs. Action potential duration is frequency dependent and when our dogs are paced at comparable rates the prolongation is less pronounced. 
Surprisingly, the increase in right ventricular action potential duration is less severe $(+21 \%)$ than that of the left ventricular action potential duration, despite a similar absolute increase in heart weight and an even higher percent increase of the right ventricular mass. Also these observations have been confirmed at the cellular level. ${ }^{21}$ This discrepancy could indicate that the changes in the electrophysiological parameters are not only related to hypertrophy and/or bradycardia. A comparison with other models is not possible because to our knowledge no report has been published describing the electrophysiologic effects in dogs with right ventricular hypertrophy. An explanation for this difference could be the different hemodynamics in the right and left ventricle. Stretch has the capability to shorten action potential duration, ${ }^{47}$ and this opposing effect may influence the amount of prolongation. Also for the comparison of the action potential duration at the two time points, this stretch induced shortening under acute atrioventricular-block circumstances may be a confounding parameter. The measured action potential duration at control (acute atrioventricular-block) could be shorter as expected on the basis of the bradycardia alone.

A second reason for the difference between the left and right ventricular action potential duration response, could be a different rate response in ventricular cells of the right and left ventricle including epicardium ${ }^{48}$ and $\mathrm{M}$ cells. ${ }^{49}$ At longer cycle lengths the prolongation of the left ventricular action potential duration is much longer than the right ventricular action potential duration. ${ }^{48}$ Studies in dogs and humans have shown the
presence of $M$ cells. 49,50

\section{Consequences of interventricular dispersion}

The consequence of the relative smaller increase in the right ventricular action potential duration compared with the left ventricular action potential duration is an increase in the interventricular dispersion under baseline conditions in the chronic atrioventricularblock dog. Interventricular dispersion is bradycardia dependent, the slower the heart rate the longer the difference between the left and right ventricular action potential duration. ${ }^{5}$ In this study, we have controlled for the cycle length, so that the observed increase in dispersion is not based on the frequency. This increased amount of interventricular dispersion could have important proarrhythmic consequences, because it is one of the recently described. ${ }^{5}$

In this study, Torsade de Pointes induction was again related to the presence of early afterdepolarizations and an impressive amount of interventricular dispersion. Following 
acute atrioventricular-block no early afterdepolarizations were present at baseline and $\triangle A P D$ amounted to $40 \mathrm{~ms}$. Administration of $d$-sotalol led to a lengthening of the repolarization parameters, appearance of early afterdepolarizations, but not to an increase in $\triangle A P D$, and no Torsade de Pointes could be induced. After 5 weeks of atrioventricular-block, $\triangle A P D$ was increased under baseline situation, while early afterdepolarizations were still not present, with one exception. In that dog Torsade de Pointes could be induced during baseline. $d$-Sotalol increased $\triangle \mathrm{APD}$ further because of its more pronounced effect on the left ventricular action potential duration and led to a higher incidence of early afterdepolarizations, and to Torsade de Pointes induction in $60 \%$ of the dogs. This means that not only the baseline values are higher in hypertrophy but that also there is a different ventricular sensitivity for $d$-sotalol. The dogs with a low $\triangle$ APD after $d$-sotalol did not develop Torsade de Pointes.

\section{Role of hypertrophy for the induction of Torsade de Pointes}

Left ventricular hypertrophy and the concomitant increase in the dispersion of intraand/or interventricular repolarization has also been associated with a higher vulnerability to other ventricular arrhythmias. ${ }^{14,20,51-52}$ This increased propensity of the hypertrophied heart is often accompanied by pathological conditions such as fibrosis, ischemia, regional differences in the degree and nature of hypertrophy, and to the functional status of the heart (heart failure). Often the pathologic condition(s) seem to be restricted to the left ventricle, but little information concerning the right ventricle is available in these situations. Therefore, it is important to emphasize that in the atrioventricular-block dog, the increased susceptibility for Torsade de Pointes seems to occur in hypertrophied myocardium in the absence of additional pathologic conditions, in which the heart has been subjected to similar increases in volume.

Regression of hypertrophy concomitant with a decrease of the electrophysiological parameters results in a markedly reduced incidence of arrhythmias to baseline. ${ }^{52}$ It would also be interesting to see whether the changes in electrophysiological parameters in our dogs can be influenced by the prevention or regression of hypertrophy. In this context it is important to mention the study of Kreher et al, ${ }^{53}$ which suggests that the adaptations leading to hypertrophy and electrophysiological changes in the aging rat heart could be two different processes which can operate independently.

Occurrence of Torsade de Pointes in dogs in the acute phase of atrioventricular-block is not impossible. Two studies have shown the occurrence of spontaneous Torsade de Pointes and polymorphic ventricular arrhythmias after administration of class III 
agents. ${ }^{54,55}$ However addition of an $\alpha$-agonist ${ }^{55}$ or a three times higher dose of almokalant was required. ${ }^{54}$ This could imply that the processes which take place in the weeks following creation of complete atrioventricular-block are more a facilitating process than an absolute requirement for the induction of Torsade de Pointes, especially in regard to the increased action potential duration and interventricular dispersion. In this regard it is of interest that in the human heart with complete atrioventricular-block Torsade de Pointes is rarely seen during acute ischemic atrioventricular-block in the setting of myocardial infarction, but much more in chronic fibrotic atrioventricular-block in adults ${ }^{56}$ and children. ${ }^{57}$ It is likely that in the latter adult patient group ventricular hypertrophy is present. ${ }^{55}$

\section{Sensitivity to d-sotalol}

Prolonged repolarization facilitates the development of early afterdepolarizations. ${ }^{20}$ The ionic mechanism of these early afterdepolarizations and of the increase in action potential duration are not completely understood. Several reports show diminishment in outward $\mathrm{K}^{+}$currents. ${ }^{13,18,58} d$-Sotalol, like most class III agents, blocks the outward $\mathrm{K}^{+}$current. Blockade of an already diminished current may explain the higher sensitivity to $d$-sotalol of the left ventricular hypertrophied muscle in atrioventricular-block dogs.

\section{Limitations}

In this study, we did not assess the time dependent behavior of the structural and electrophysiological changes. Because we measured only at two time points, it is possible that the adaptations were not fully completed. Earlier studies comparing dogs at different time intervals with a minimum of 2 weeks, however, showed that there is no relation with duration of atrioventricular-block and total heart weight. ${ }^{45,46}$ We had similar findings comparing the obtained heart weights at 20 weeks with 9 weeks atrioventricular-block. In relation to the electrophysiological effects, the response to $d$-sotalol does not change in time, when we compare the experiments that have been started after 2 weeks atrioventricular-block. ${ }^{4,5}$ This suggests that the alteration responsible for the facilitated Torsade de Pointes induction is already present at two weeks. The time period between 0 and 2 weeks will be the subject of further investigations.

A second limitation could be the (reproducible) quality of the signals and the random placement of the endocardial monophasic action potentials during these experiments. Especially, directly after creating atrioventricular-block it is technically difficult to acquire good monophasic action potential signals. Perhaps this is due to the stretch on 
the ventricular wall. Random placement of the monophasic action potential-catheters ignores possible intraventricular differences in action potential duration. However we have shown that the intraventricular difference at baseline and after $d$-sotalol is always much smaller (only $20 \%$ ) than the interventricular dispersion. ${ }^{5}$

Finally, we have the difficulty that we are not yet able to measure the changes that occur during the pacing train leading to induction of Torsade de Pointes. We know that changes in frequency lead to augmentation of early afterdepolarizations, and to occurrence of triggered ectopic beats. The dynamicity of the changes in $\triangle A P D$ can however not be assessed at this time.

\section{Possible clinical implications}

Extrapolation from data derived from experimental animals to the human setting should always be done with great caution. It is known that many different arrhythmia mechanisms may be generated in the hypertrophied myocardium resulting in symptomatic arrhythmias and death. ${ }^{18-20}$ We can hypothesize that patients with ventricular hypertrophy are more prone to the proarrhythmic effects of the Torsade de Pointes type during treatment with repolarization prolonging agents than patients without hypertrophy. A first step to get better informed about that possibility could be to stratify patients with Torsade de Pointes not only to QT interval, QT dispersion and heart rate but also to localization, degree and/or etiology of ventricular hypertrophy.

\section{General conclusions}

The electrical remodeling (non-uniform ventricular prolongation of action potential duration and different sensitivity for $d$-sotalol) occurring after chronic atrioventricularblock predisposes the heart for acquired Torsade de Pointes, while the structural changes (physiological hypertrophy) are successfully recruited to maintain cardiac function.

\section{Acknowledgments}

This study was supported in part by grants from the Dutch Heart Foundation ( $\$ 94010$ ), the Bekales Foundation, and the Wynand N. Pon Foundation. The authors thank Dr. P Schiffers (Pharmacology), Mr. Th. van der Nagel and Mr. P. Willemsen (Physiology) and Mr. E. Wijers (Pathology) for their contribution in collecting and analyzing the data. The authors also like to thank Bristol Meyers Squibb for providing $d$-sotallol and Bakken Research Center, Medtronic for the epicardial electrodes. 


\section{References}

1. Gorgels APM, Beekman HDM, Brugada P, Dassen WRM, Richards DAB, Wellens HJJ. Extrastimulus related shortening of the first postpacing interval in digitalis induced ventricular tachycardia. JAm Coll Cardiol 1984; 1:840-857.

2. Vos MA, Gorgels APM, Leunissen HDM, Wellens HJJ. Flunarizine allows differentiation between mechanisms of arrhythmias in the intact heart. Circtilation $1990 ; 81: 343-349$.

3. de Groot SHM, Vos MA, Gorgels APM, Leunissen HDM, van der Steld BJ, Wellens HJJ. Combining monophasic action potential recordings with pacing to demonstrate delayed afterdepolarizations and triggered arrhythmias in the intact heart. Value of the diastolic slope. Circulation 1995 ; 92: $2697-2704$.

4. Vos MA, Verduyn SC, Gorgels APM, Lipscei GC, Wellens HJJ. Reproducible induction of early afterdepolarizations and torsade de pointes arrhythmias by $\mathrm{d}$-sotalol and pacing in the dog with chronic atrioventricular block. Cinculation 1995; 91: 864-872.

5. Verduyn SC, Vos MA, Zande van der J, Hulst van der FF, Wellens HJJ. Role of interventricular dispersion of repolarization in acquired Torsade de Pointes arrhythmias: reversal by magnesium. Cardiovasc Res 1997; 34: 453-463.

6. Turina $M$, Bussmann WD, Krayen buhl HP. Contractility of the hypertrophied canine heart in chronic volume overload. Cardiovasc Res 1969; 3: 486-495.

7. Wusten B, Flameng W, Winkler B, Schaper W. Role of cardiac contractility in bypertrophy from chronic volume loading. Cardiovasc Res 1977; 11:132-140.

8. Newman WH. Contractile status of hypertrophied left ventricle in long standing volume overload. Am J Physiol 1978; 234: H88-H93.

9. Kannel WB, Gordon T, Offutt D. Left ventricular hypertrophy by electrocardiogram. Prewalence incidence and mortality in the Framingham study. Am Intern Med 1969; 71: 89-101.

10. Kannel WB and Sorlie P. Left ventricular hypertrophy in hypertension: prognostic and pathogenetic implications. The Fram ingham study in Strauer R ed. 'The heart in hypertension', Berlin: Springer Verlag 1981: 223-242.

11. Messerli FH, Grodzicki T. Hypertension, left ventricular hypertrophy, ventricular and sudden death. Eur Heart J 1992: 13: D66-D69.

12. Klein $\mathrm{RC}$. Ventricular arrhythmias in aortic valve disease: analysis of 102 patients. $A \mathrm{~m} . / \mathrm{Cardiol}$ 1984; $53: 1079-1083$

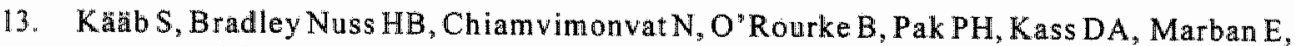
Tomaselli GF. lonic mechan ism of action potential prolongation in ventricular myocytes from dogs with pacing induced heart failure. Circ Res 1996; 78: 262-273.

14. Sabbah HN, Goldberg AD, Schoels W, Kono T, Webb C, Brachmann J, Goldstein S. Spontaneous and ind ucible ventricular arrhythmias in a canine model of chronic heart failure: relation to haemodynamics and sympathoadrenergic activation. Eur Heart $J$ 1992; 13: 1562-1572.

15. Ben-David J, Zipes DP, Ayers GM, Pride HP. Canine left ventricular hypertrophy predisposes to ventricular tachycardia induction by phase 2 early afterdepolarizations after administration of Bay K 8644. J Am Coll Cardiol 1992; 20: 1576-1584.

16. Martins JB, Kim W, Marcus ML. Chronic hypertension and left ventricular hypertrophy facilitate induction of sustained ventricular tacycardia in dogs 3 hours after left circumflex coronary artery acclusion. J Am Coll Cardiol 1989; 14: 1365-1373.

17. Cameron IS, Myerburg RJ, Wong SS, Gaide MS, Epstein K, Alvarez TR, Gelband H, Guse PA, Bassett AL. Electrophysiologic consequences of chronic experimentally induced left ventricular pressure overload. J Am Coll Cardiol 1983; 2: 481-487. 
18. Tomaselli GF, Beuckelmann DJ, Calkins HG, Berger RD, Kesssler PD, Lawrence JH, Kass D, Feldman AM, Marban E. Sudden cardiac death in heart failure. The role of abnomal repolarization. Circulation 1994: $90: 2534-2539$.

19. Pye MP, Cobbe SM. Mechanisms of ventricular arrhythmias in cardiac failure and hypertrophy. Cardiovasc Res 1992; 26: 740-750.

20. Aronson RS, Ming Z. Cellular mechanisms of arrhythmias in hypertrophied and failing myocardium. Circulation 1993; 87: VII76-VII83.

21. Volders PGA, Sipido KR, Vos MA, Kulscár A, Verduyn SC, Wellens HJJ. Cellular basis of biventricular hypertrophy and arrhy thmogenesis in dogs with chronic complete atrioventricular block and acquired torsade de pointes. Accepted for publication in Circulation.

22. Vatner DE, Sato N, Kiuchi $\mathrm{K}$, Shamnon $\mathrm{RP}$, Vatner SF. Decrease in myocardial ryanodine receptors and altered excitation-contraction coupling early in the development of heart failure. Circulation 1994; $90: 1423-1430$.

23. Schreuder JJ, van der Veen FH, van der Velde ET, Delahaye F, Alfieri $O$, Jegaden O, Lorusso R, Jansen IRC, wan Ommen $V$, Finet $G$, Wellens $H J J$. Beat-to-beat analysis of left ventricular pressurevolume relation and stroke volume by conductance catheter and aortic modelflow in cardiomyoplasty patients. Circulation 1995; 91: 2010-2017.

24. Nussberger $J, B$ runner DB, Waeber B, Brunner HR. True versus immunoreactive angiotensin II in human plasma. Hypertension 1985; 7: 11-17.

25. Rosmalen FM, Tan AC, Tan HS, Benraad TJ. A sensitive radioimmunoassay of atrial natriuretic peptide in human plasma, using a tracer with an immobilized glycouril agent. Clinica Chemica 1987; 165: 331-340.

26. Kubasik NP, Warren $\mathrm{K}$, Sine HE. Evaluation of a new commercial radioassay kit for aldosterol using an iodinated tracer. Clin Biochem 1987; 12; 59-61.

27. Van der Hoom $\mathrm{K}$, Boomsma $\mathrm{F}$, Man in "t Veld AJ, Schalekamp MADH. Determination of catecholamines in human plasma by high-performance liquid chromatography: comparison between a new method with fluorescence detection and an established method with electrochemical detection. $J$ Chromatography 1989; 487: 17-28.

28. Weber KT, Pick R, Silver MA, Moe GW, Janicki JS, Zucker IH, Armstrong PW. Fibrillar collagen and remodeling of dilated canine left ventricle. Circulation 1990; 82: 1387-1401.

29. Rakusan $\mathrm{K}$, Flanagan MF, Geva T, Southern J, Va n Praagh R. Morphometry of human coronary capillaries during nomal growth and the effect of age in left ventricular pressure-overload hypertrophy. Circulation 1992; 86: 38-46.

30. Sabbah HN, Sharov VG, Lesch M, Goldstein S. Progression of heart failure: a rolle for interstitial fibrosis. Mol Cell Biochem 1995; 147: 29-34.

31. Sambrook J, Fritsch EF, Maniatis T. Molecular cloning: A Laboratory Manual, 2nd ed. Cold Spring Habor, NY: Cold Spring Harbor Laboratory Press 1989.

32. Feinberg AP, Vogelstein B. A technique for radiolabeling DNA restriction endonuclease fragments to high specific activity. Annal Biochem $1983 ; 132: 6-13$.

33. Holmer SR, Riegger AJG, Notheis WF, Kromer EP, Kochsiek K. Hemodynamic changes and renal plasma flow in early heart failure: implications for renin, aldosterone, norepinephrine, atrial natriuretic peptide and prostacyclin. Basic Res Cardiol 1987; 82: 101-108.

34. Moe GW, Stopps TP, Angus C, Forster C, de Bold A, Armstrong PW. Alterations in serum sodium in relation to atrial natriuretic factor and other neuroendocriene variables in experimental pacinginduced heart failure. J Am Coll Cardiol 1989; 13: 173-179. 
35. Spinale FG, Holzgrefe HH, Mukherjee R, Arthur SR, Child MJ, Powell JR, Koster WH. LV and myocyte structure and function after early recovery from tachycardia induced cardiomyopathy. $A m$ IPhysiol 1995; 268: H836-H847.

36. Perreault $\mathrm{CL}$, Shannon $\mathrm{RP}, \mathrm{K}$ omamura $\mathrm{K}$, Vatner $\mathrm{SF}$, Morgan JP. Abnormalities in intracellular calciurn regulation and contractile function in myocardium from dogs with pacing induced heart failure. I Clin lnvest 1992; 89: 932-938.

37. Williams RE, Kass DA, Kawagoe $Y$, Pak P, Tumin RS, Shah R, Hwang A, Feldman AM. Endomyocardial gene expression during development of pacing tachycardia-induced heart failure in the dog. Circ Res 1994; 75: 615-623.

38. McDonald KM, Francis GS, Carlyle PF, Hauer K, Matthews J, Hunter DW, Cohn JN. Hemodynamic, left ventriuclar structural and hormonal changes after discrete myocardial damage in the dog. IAm Coll Cardiol 1192; 19:460-467.

39. Hittinger $L$, Patrick T, Ihara T, Hasebe N, Shen YT, Kalthof B, Shannon RP, Vatner SF. Exercise induced cardiac dysfunction in both moderated, compensated and severe hypertrophy. Circulation 1994; 89: 2219-2231.

40. Hittinger L, Shannon RP, Bishop SP, Gelpi RJ, Vatner SF. Subendomyocardial exhaustion of blood flow reserve and increased fibrosis in conscious dogs with heart failure. Circ Res 1989; 65:971-980.

41. Hasebe N, Hittinger L, Kohin S, Shen YT, Graham RM, Vatner SF. Effects of hypertrophy on left atrial and ventricular compliance and plasma. ANF levels in conscious dogs. Am J Physiol 1995; 268: H781-H787.

42. Sabbah $\mathrm{HN}_{\text {, Stein }} \mathrm{PD}$, Kono $\mathrm{T}$, Gheorghiade $\mathrm{M}$, Levine TB, Jafr $\mathrm{S}$, Hawkins ET, Goldstein $\mathrm{S}$. A canine model of chronic heart failure produced by multiple sequential coronary microembolisations. Am J Physiol 1991; 260: H1379-1384.

43. Omens JH, Covell JW. Transmural distribution of myocardial tissue growth induced by volume overload hypertrophy in the dog. Circulation 1991; 84: 1235-1245.

44. Urabe Y, Hamada Y, Spinale FG, Carabello BA, Kent RL, Cooper IV G, Mamn DL. Cardiocyte contractile performance in experimental biventricular volume-overload bypertrophy. Am JPhysiol 1993; 264: H1615-H1623.

45. Starzl TE, Gaertner RA. Chronic heart block in dogs. A method for producing experimental heart faillure. Circulation $1955 ; 12: 259-270$.

46. Brockman SK. Cardiodynamics of complete heart block. Am J Cardiol 1965; 16: 72-83.

47. Franz MR, Burkhoff D, Yue DT, Sagawa K. Mechanically induced action potential changes and arthythmia in isolated and in situ canine hearts. Cardiovasc Res 1989; 23:213.223.

48. Di Diego JM, Sun ZQ, Antzelevitch C. Ito and action potential notch are smaller in left versus right canine ventricular epicardium. Am $J$ Physiol 1996; H548-H561.

49. Antzelevitch C, Sicouri S. Clinical relevance of cardiac arrhythmias generated by afterdepolarizations. Role of $M$ cells in the generation of $U$ waves, triggered activity and Torsade de Pointes. $J . A m$ Coll Cardiol 1994; $23: 259-277$.

50. Drouin $\mathrm{E}$, Charpentier F, Gauthier $\mathrm{CL}_{\text {r, }}$ Laurent $\mathrm{K}$, Le Marec $\mathrm{H}$. Electrophysiological characteristics of cells spanning the left ventricular wall of human heart evidence for the presence of M cells. $J A m$ Coll Cardiol 1995; 26: 185-192.

51. Kowey PR, Friehling TD, Sewter J, WU Y, Sokil A, Paul J, Nocella J. Electrophysiological effects of left ventricular hypertrophy. Effect of calcium and potassium channel blockade. Circulation 1991; 83: 2067-2075.

52. Rials SJ, WU Y, Ford N, Pauletto FJ, Abramson SV, Rubin AM, Marinchak RA, Kowey PR. Effect of left ventricular hypertrophy and its regression on ventricular electrophysiology and vulnerability to inducible arrhythmia in the feline heart. Circulation 1995; 91: 426-430. 
53. Kreher $P$, Ristori MT, Verdetti J. Effects of chronic angiotensin I-converting enzyme inhibition on the relations between ventricular action potential changes and myocardial hypertrophy in aging hearts. J Cardiovasc Pharmacal 1995; 25: 75-80.

54. Duker GD, Linhardt GS, Rahmberg M. An animal model for studying class III induced proarthythmias in the halothane-anesthetized dog. J Am Coll Cardiol 1994:326A. (abstract)

55. Buchanan LV, Gibson K. Characterization of monomorphic and polymorphic tachycardia in a caaine model of proarthythmia. Circulation 1994; 90: 248.(abstract)

56. Kurita T, Ohe T, Marui N, Aihara N, Takaki H, Kamakura S, Matsutuisa M, Shimomura K. Bradycardia induced abnormal QT-prolongation in patients with complete AV block with Torsade de Pointes. Am J Cardiol 1992; 69: 628-633.

57. Gladman G, Davies AM, Fogelman R, Hamilton RW, Gow RM. Torsade de Pointes, acquired complete heart block and inappropriately long QT in childhood. Com $J$ Cardiol 1996; 12, 683-685.

58. Furukawa T, Myerburg RJ, Furukawa N, Kimura S, Bassett AL. Metabolic inhibition of ICa L and IK differs in feline left ventricular hypertrophy. Am J Physiol 1994; 266: H1121-HI131. 


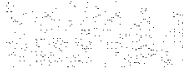

a

a

हो

$\therefore$

$\therefore$

$\therefore \quad \therefore$ 


\section{Chapter 6}

\section{Adaptations to preserve cardiac function predispose the hypertrophied canine heart for delayed afterdepolarization-dependent arrhythmias}

Marieke de Groot

Marc Vos

Jet Leunissen

Marieke Schoenmakers

Hein Wellens

Abstract presented at the American Heart Association 1997 (Circulation 1997: 96: (239-I240)

Submitted for publication 


\section{Abstract}

Introduction: In dogs, chronic complete atrioventricular-block leads to biventricular hypertrophy, prolonged ventricular repolarization and an increased sensitivity to early afterdepolarization-dependent triggered arrhythmias. The effect of atrioventricularblock on susceptibility to delayed afterdepolarizations is not well known. Additionally it is unknown how the occurrence of arrhythmias relates to changes in contractile function, especially when a sudden increase in contractility is required.

Methods: We investigated the effect of ventricular pacing with a variable number (1-68) of stimuli (interval $300 \mathrm{~ms}$ ) on post-pacing potentiaton of left ventricular function $(+\mathrm{LV}$ $\mathrm{dP} / \mathrm{dt}$ ) in relation to induction of delayed afterdepolarizations (registered with a monophasic action potential catheter) and triggered arrhythmias. Ten dogs were tested directly after the creation of complete atrioventricular-block (to), whereas 14 dogs were tested at 6 weeks of atrioventricular-block ( $t 6)$.

Results: During idioventricular rhythm, the $+\mathrm{LV} \mathrm{dP} / \mathrm{dt}$ increased from $992 \pm 284 \mathrm{~mm} \mathrm{Hg} / \mathrm{s}$ at to to $1726 \pm 332 \mathrm{~mm} \mathrm{Hg} / \mathrm{s}$ at $\mathrm{t} 6(\mathrm{p}<0.05)$. Increasing the number of stimuli resulted in an increase in post-pacing-potentiation both at to and $t 6$. Post-pacing $+\mathrm{LV} \mathrm{dP} / \mathrm{dt}$ showed a maximum after $5-13$ stimuli at both times, but was higher at $\mathrm{t} 6(\mathrm{p}<0.05)$. In addition, at $\mathrm{t} 6$, delayed afterdepolarizations occurred in 8 of 10 dogs and triggered ectopic beats, while they were not seen at to. Interestingly, their occurrence also depended on the number of stimuli and occurred at the highest post-pacing $+\mathrm{LV} \mathrm{dP} / \mathrm{dt}$. At t6, increasing the $+\mathrm{LV} \mathrm{dP} / \mathrm{dt}$ (to $2075 \pm 534 \mathrm{~mm} \mathrm{Hg} / \mathrm{s}, \mathrm{p}<0.05$ ) and post-pacing-potentiation with ouabain $(20 \mu \mathrm{g} / \mathrm{kg})$ resulted in an increased severity of arrhythmias, while ryanodine (10 $\mu \mathrm{g} / \mathrm{kg}$ ) decreased $+\mathrm{LV} \mathrm{dP} / \mathrm{dt}$ (from $2327 \pm 618$ to $759 \pm 185 \mathrm{~mm} \mathrm{Hg} / \mathrm{s}, \mathrm{p}<0.05$ ) and post-pacing-potentiation, which suppressed delayed afterdepolarization-dependent triggered arrhythmia. Prolonged rapid ventricular pacing at a fixed rate $(100-120$ beats $/ \mathrm{min}$, for 3 minutes) decreased both pre- and post-pacing $+\mathrm{LV} \mathrm{dP} / \mathrm{dt}$, which was accompanied by suppression of triggered arrhythmias.

Conclusions: Intrinsically enhanced contractile function in chronic, complete atrioventricular-block predisposes the hypertrophied heart to delayed afterdepolarization dependent triggered ventricular arrhythmias. The occurrence of these arrhythmias is promoted by positive inotropic interventions. Interventions that depress contractile function are accompanied by suppression of these arrhythmias. 


\section{Introduction}

Hypertrophy is the adaptive mechanism of the heart to mechanical overload of any cause and aimed at maintaining cardiac function. Independent of its cause and coexisting factors, left ventricular hypertrophy has been associated with an increased incidence of ventricular arrhythmias and sudden (arrhythmic) death. ${ }^{1-3}$

Whether the hypertrophied heart is able to maintain its contractile performance, remains controversial. Experimental studies using pressure overload hypertrophy, have demonstrated depressed, ${ }^{4,5}$ normal ${ }^{6}$ or supranormal ${ }^{7}$ baseline myocardial function. Similar conflicting results have been obtained following volume overload. ${ }^{6,8-13}$ Dynamic cardiac function has been studied, using: frequency potentiation, ${ }^{14-16}$ post-extrasystolic potentiation ${ }^{17-19}$ and mechanical restitution. ${ }^{17,18}$ In vitro, these methods have been coupled to calcium transient recordings. 20,21

The altered contractile function of hypertrophied and failing hearts has been associated with adaptations in intracellular calcium handling. ${ }^{20,21}$ Simultaneously, intracellular calcium overload has been associated with triggered activity arising from delayed afterdepolarizations. ${ }^{22,23}$ Thus, based on intracellullar calcium handling, a common etiology between functional adaptations and arrhythmias can be expected.

In dogs, creation of complete atrioventricular-block leads to biventricular hypertrophy. $9,11-13,24-26$ According to autopsy and neurohumoral data, this process seems to have stabilized within 6 weeks. ${ }^{25}$ Electrophysiologically, chronic complete atrioventricular-block is associated with a prolonged repolarization and an increased sensitivity to early afterdepolarization dependent Torsade de Pointes arrhythmias. ${ }^{26}$

The effect of atrioventricular-block induced hypertrophy on susceptibility to delayed afterdepolarization dependent arrhythmiass is not known. Additionally, it is unknown what relation (if present) exists between changes in contractile requirements during complete atrioventricular-block and delayed afterdepolarization induced arrhythmias. Therefore, we investigated the effect of a variable number of stimuli on post-pacing occurrence of delayed afterdepolarizations and triggered arrhythmias in relation to the post-pacing left ventricular contractile function $(+\mathrm{LV} \mathrm{dP} / \mathrm{dt})$ in the hypertrophied heart of the dog with chronic complete atrioventricular-block. 
Table 1: overview of the dogs used in the study and of the autopsy data

\begin{tabular}{|c|c|c|c|c|c|c|c|c|c|}
\hline Dog \# & Weight & $\begin{array}{l}\text { Heart } \\
\text { Weight }\end{array}$ & $\begin{array}{l}\text { H/BW } \\
\text { ratio }\end{array}$ & $\begin{array}{l}\text { Days } \\
\text { AVB }\end{array}$ & $\begin{array}{l}\text { Heart } \\
\text { weight }\end{array}$ & $\begin{array}{l}\text { LY } \\
\text { Mass }\end{array}$ & $\begin{array}{l}\text { RV } \\
\text { Mass }\end{array}$ & $\begin{array}{l}\mathbf{L V} \\
\mathbf{H} / \mathbf{B W}\end{array}$ & $\begin{array}{l}\text { RV } \\
\text { H/BW } \\
\end{array}$ \\
\hline 10 & 24 & 309 & 12.9 & 41 & 281 & 159 & 66 & 6.6 & 2.8 \\
\hline 2 & 30 & 300 & 10.0 & 40 & & & & & \\
\hline $30, \mathrm{CP}$ & 40 & 390 & 9.8 & 58 & 385 & 165 & 98 & 4.1 & 2.5 \\
\hline $4 \mathrm{O}, \mathrm{CP}$ & 29 & 265 & 9.1 & 47 & & & & & \\
\hline $50, \mathrm{CP}$ & 22 & 265 & 12.0 & 40 & & & & & \\
\hline $60, \mathrm{CP}$ & 33 & 307 & 9.3 & 44 & & & & & \\
\hline $70, \mathrm{CP}$ & 26 & 275 & 10.6 & 50 & & & & & \\
\hline $8 \dagger$ & 26 & 348 & 13.4 & (29) & 338 & 197 & 69 & 7.6 & 2.7 \\
\hline $9+$ & 22 & 225 & 10.2 & (31) & 207 & 121 & 54 & 5.5 & 2.5 \\
\hline $10 \uparrow$ & 29 & 293 & 10.1 & (23) & 286 & 163 & 59 & 5.6 & 2.0 \\
\hline 11 & 25 & 240 & 9.6 & 68 & & & & & \\
\hline 12 & 26 & 275 & 10.6 & 50 & & & & & \\
\hline 13 & 25 & 253 & 10.1 & 67 & & & & & \\
\hline $14 \mathbb{R}$ & 30 & 283 & 9.4 & 69 & 277 & 153 & 64 & 5.1 & 2.1 \\
\hline $15 \mathrm{R}$ & 22 & 306 & 13.9 & 126 & & & & & \\
\hline $16 \mathrm{R}$ & 31 & 376 & 12.1 & 78 & & & & & \\
\hline $17 \mathrm{R}$ & 28 & 320 & 11.4 & 84 & & & & & \\
\hline Mean & 28 & 296 & 10.9 & 56 & 296 & 160 & 68 & 5.8 & 2.4 \\
\hline SD & 5 & 45 & 1.5 & 25 & 60 & 24 & 15 & 1.2 & 0.3 \\
\hline
\end{tabular}

Dogs \#1-7 studied at 0 and 6 weeks of atrioventricular-block (AVB), $†(\# 8-10)$ died before restudy, \#11-17 only at 6 weeks, O: ouabain, CP: continuous pacing, R: ryanodine at 6 weeks, Weight: kg, Heart Weight: $\mathrm{g}, \mathrm{H} / \mathrm{BW}$ ratio: heart to body weight ratio $(\mathrm{g} / \mathrm{kg}), \mathrm{LV} / \mathrm{BW}$ : left ventricle to body weight ratio $(\mathrm{g} / \mathrm{kg}), \mathrm{RV} / \mathrm{BW}$ : right ventricle to body weight ratio $(\mathrm{g} / \mathrm{kg})$, Days AVB: duration of atrioventricular-block at sacrifice or demise 


\section{Methods}

All experiments were performed in accordance with the "Guiding principles in the care and use of laboratory animals" (NIH publication \#86-23, 1996) as approved by the American Physiological Society and under the Dutch Law on Animal Experimentation and The European Directive for the Protection of Vertebrate Animals used for Experimental and other Scientific Purposes. All experiments were approved by the Animal Investigation Committee of the Maastricht University.

\section{General}

A total of 17 anesthetized mongrel dogs of either sex (mean body weight of $28 \pm 5 \mathrm{~kg}$ ) were used for this study (table 1). In 13 of these 17 dogs (\#1 to \#13, table 1) the effect of complete atrioventricular-block on left ventricular function in relation to inducibility of post-pacing ectopic beats and delayed afterdepolarizations was studied at acute and/or chronic ( 6 weeks) atrioventricular-block. For this purpose, 10 of the 13 dogs (\#1 to \#10) were studied immediately after the creation of complete atrioventricular-block and were scheduled to be restudied after 6 weeks. Unfortunately, 3 of these 10 dogs ( $\# 8$ to \#10) died before restudy, of which one suddenly from an unknown cause. Therefore, 3 dogs (\#11 to \#13) with an identical duration of atrioventricular-block, were added to the study and tested at 6 weeks of atrioventricular-block only. In some of these dogs, the effects of ouabain ( $20 \mu \mathrm{g} / \mathrm{kg} / 1$ minute, $\mathrm{n}=6)$ and fixed rate pacing (at the previous sinus rhythm frequency, $n=5$ ) on left ventricular contractile function, ectopic beat and delayed afterdepolarization induction were studied both at the time of acute and chronic complete atriowentricular-block (table 1). An additional 4 dogs (\#14 to \#17) were used to study the effect of ryanodine $(10 \mu \mathrm{g} / \mathrm{kg} / 10$ minutes) on the induced delayed afterdepolarizations and ectopic beats at 6 weeks of atrioventricular-block (table 1 ).

\section{Anesthesia}

After overnight fasting, premedication $(0.2 \mathrm{ml} / \mathrm{kg})$ was given intramuscularly with a drug mixture containing $10 \mathrm{mg}$ oxycodan $\mathrm{HCl}, 1 \mathrm{mg}$ acepromazine and $0.5 \mathrm{mg}$ atropine sulfate per $\mathrm{ml}$. Thirty minutes later, anesthesia was induced by sodium pentobarbital ( $20 \mathrm{mg} / \mathrm{kg}$ I.V.). The dogs were artificially ventilated (Pulmomat respirator, Dräger), through a cuffed endotracheal tube with a mixture of oxygen, nitrous oxide and halothane (vapor concentration $0.5-1.0 \%$ ). Ventilation was adjusted on the basis of continuous monitoring of the carbon dioxide concentration of the expired air. During the experiments, a thermal mattress was used to maintain adequate body temperature. Proper care was taken during 
and after the experiments, including the administration of antibiotics (1000 mg ampicillin) and analgesics $(0.015 \mathrm{mg} / \mathrm{kg}$ buprenorfine I.M. postoperatively). Drugs were administered through a cannula in a cephalic vein.

\section{Creation of complete atrioventricular-block}

To induce complete atrioventricular-block, a right-sided thoracotomy was performed through the fourth or fifth intercostal space and the pericardium was opened. An epicardial screw-in electrode (Bakken Research Center, Maastricht, The Netherlands) was placed at the apex of the left ventricular. The lead of the epicardial electrode was tunneled subcutaneously and exteriorized in the neck of the animal. After the creation of complete atrioventricular-block by injecting formaldehyde (37\%) in the AV-node, ${ }^{27}$ the thorax was closed.

\section{Experiments}

All experiments were performed under sterile conditions. During the experiments a six channel ECG was recorded. The dogs were catheterized to record left ventricular and right ventricular monophasic action potential-signals and left ventricular pressure curves. All signals were simultaneously registered on a monitor and stored on the hard disc of the computer. With a computerized QRS detection system, the values of the RR intervals were instantaneously visualized on a monitor.

The monophasic action potential catheters (Steerable Franz ${ }^{\mathrm{TM}}$ Combination Catheter, EP technologies) were randomly placed in the ventricles through the femoral artery and vein, under fluoroscopic guidance. ${ }^{28}$ The monophasic action potential-signals were amplified using a custom built DC-coupled differential amplifier with a $20 \mathrm{mV}$ calibration pulse. The offset of this amplifier is variable and can be adjusted to the recorded signal. A detailed description of the methodology was previously published. ${ }^{29}$ The monophasic action potential signals were checked for stability, a constant configuration and shape, and a minimally accepted amplitude of $15 \mathrm{mV}$ before the experiment started. $^{28,29}$

The left ventricular pressure curve was recorded using a single micromanometer transducer catheter ( $7.5 \mathrm{~F}$, Sentron, Roden, The Netherlands), ${ }^{30}$ which was positioned in the left ventricle via the carotid artery with the aid of fluoroscopy. Prior to insertion, the pressure sensors were emerged in a $37^{\circ} \mathrm{C} 0,9 \% \mathrm{NaCl}$ solution to minimize temperature drift. The Sentron interface (Type No 811-000/A) was used for calibration of the pressure transducer. ${ }^{30}$ All signals were sampled at a rate of $1 \mathrm{kHz}$ per signal. 
After completion of the experiments, all catheters were withdrawn and the vessels were sutured. The animals were allowed to recover from anesthesia and returned to their quarters.

\section{Pacing protocol}

After complete instrumentation of the dog, programmed electrical stimulation was performed. The ventricle was stimulated from the right ventricular monophasic action potential with a programmable stimulator having a synchronizing circuit. The heart was stimulated at $1 \mathrm{~mA}$ (being approximately 4 times diastolic threshold), with an interstimulus interval of $300 \mathrm{~ms}$. The pacing trains consisted of $1,3,5,8,10,13,33$ and 68 stimuli $(n=8)$. Between the pacing trains, the heart was allowed enough time to regain its prepacing rhythm. The effect of this pacing protocol on left ventricular function and the inducibility of delayed afterdepolarizations and ectopic beats was studied during: 1) sinus rhythm, 2) acute atrioventricular-block and 3) chronic ( 6 weeks of) complete atrioventricular-block. During sinus rhythm, programmed stimulation was performed only during control circumstances, while both at acute and chronic atrioventricular-block the protocol was repeated during different interventions.

\section{Interventions}

1) Ouabain: After the pacing protocol was performed during control (non-drug) circumstances, a 1 minute bolus of $20 \mu \mathrm{g} / \mathrm{kg}$ ouabain was administered intravenously in 6 dogs who were tested both during acute and chronic atrioventricular-block (table 1 , dog \#1, \#3 to \#7). The pacing protocol was repeated 10 minutes after the bollus of ouabain.

2) Fixed rate pacing: After the pacing protocol was performed during idioventricular rhythm, the above described pacing protocol was repeated during a continuously paced, fixed rate rhythm with a paced cycle length of approximately $100-120$ beats per minute, equal to the previously existing sinus rhythm, both at acute and chronic complete atrioventricular-block (table 1, dogs \#3 to \#7). For this purpose, the ventricle was paced from the epicardial electrode at twice diastolic threshold with a pulse width of $2 \mathrm{~ms}$. Fixed rate pacing was interrupted by the short pacing trains (1-33 stimuli, interval 300 ms) and restarted immediately after this short pacing train with a recovery interval equaling the cycle length of fixed rate pacing.

3) Ryanodine: At chronic atrioventricular-block a 10 minute bolus of ryanodine (10 $\mu \mathrm{g} / \mathrm{kg}$ ) was administered in 4 selected dogs (table $1, \# 14$ to \#17) that showed delayed afterdepolarizations and triggered arrhythmias. This was done to investigate afterdepo- 
larizations as the mechanism underlying the ectopic beats, to exclude the registration of artifacts with the monophasic action potential and to study the relation between alterations in cardiac contractile function and arrhythmias.

\section{Data analysis}

Using a software program, ${ }^{29}$ the data were analyzed off line. To correlate the functional adaptations after creation of complete atrioventricular-block with the occurrence of triggered activity, the following parameters were measured in each experiment: 1) cycle length before pacing and of the first post-pacing interval, 2) left ventricular end systolic pressure, left ventricular end diastolic pressure and the duration of the left ventricular and right ventricular action potential at $100 \%$ repolarization before each pacing train, 3) the $+\mathrm{LV} \mathrm{dP} / \mathrm{dt}$ max before each pacing train and of 3 post-pacing beats being spontaneous, ectopic or paced.

In all experiments, the presence of delayed afterdepolarizations in the monophasic action potential signals was quantified, before, during and after pacing. The presence of a delayed afterdepolarization in the monophasic action potential was defined as a depolarizing afterpotential starting after the complete repolarization. ${ }^{29,31,32}$ Only delayed afterdepolarizations with a diastolic slope of $\geq 10 \mathrm{mV} / \mathrm{s}$ were considered in our analysis. ${ }^{29}$ Monophasic action potential signals were analyzed based on stability and quality of the signal. For the purpose of this study, a ventricular ectopic beat was defined as a ventricular activation with a post-pacing interval shorter than $600 \mathrm{~ms}$. Ventricular tachycardia was defined as the presence of $\geq 5$ consecutive ectopic beats.

Figure 1 at the right page: The effect of increasing the number of stimuli on postpacing potentiation $\left(P P P_{*}+L V d P / d t\right)$ and inducibility of ectopic beats (EBs) at sinus rhythm, acute and chronic atrioventricular-block (AV-block) during control circumstances.

In all panels, the number of ventricular stimuli ( $\mathrm{nVs}$, interstimulus interval $300 \mathrm{~ms}$ ) are indicated on the horizontal axis. In panels 1 and 2, the postpacing $+\mathrm{LV} \mathrm{dP/dt}$ is given on the vertical axis, while in panel 3 the percentage of pacing trains that were followed by the occurrence of $\geq 1$ ectopic beat is indicated. Data are presented as mean $\mathrm{SEM}, * \mathrm{p}<0.05$, compared to $\mathrm{nVs}=1$.

Panel 1 illustrates the effect of the varaible $n V s$ on postpacing $+L V \mathrm{dP} / \mathrm{dt}$ when pacing is performed during sinus thythm. Initially, an increase in $\mathrm{nV}$ s is accompanied by an increase in postpacing $\mathrm{LV} \mathrm{dP} / \mathrm{dt}$, which reaches a maximum after 8 stimuli and than declimes.

In panel 2 similar curves are depicted, but now pacing is performed during idioventricular thythm at acute AV-block and chronic AV-block. The postpacing $+\mathrm{LV}$ dP/dt curve at chronic AV-block is significantly higher that at acute AV-block. This coincides with the occurrence of EBs when pacing is performed at chronic AV-block (panel 3). The highest incidence of EBs corresponds with the moment postpacing +LV $\mathrm{dP} / \mathrm{dt}$ was expected to be maximal (between $5-13$ stimuli). 


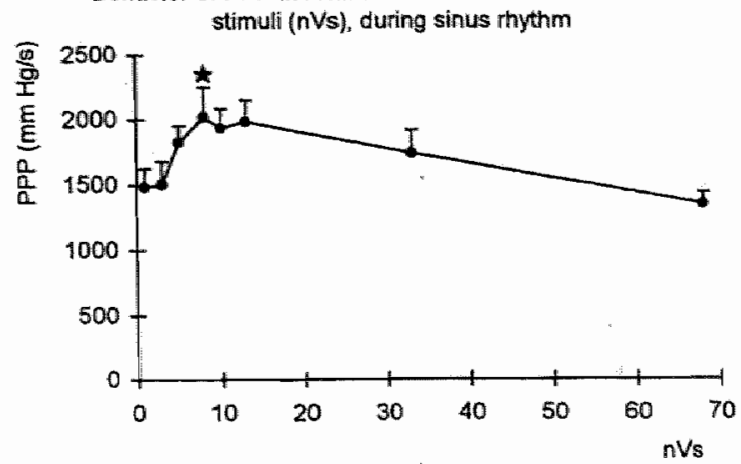

2 Behavior of PPP in relation to the number of stimuli (nVs)

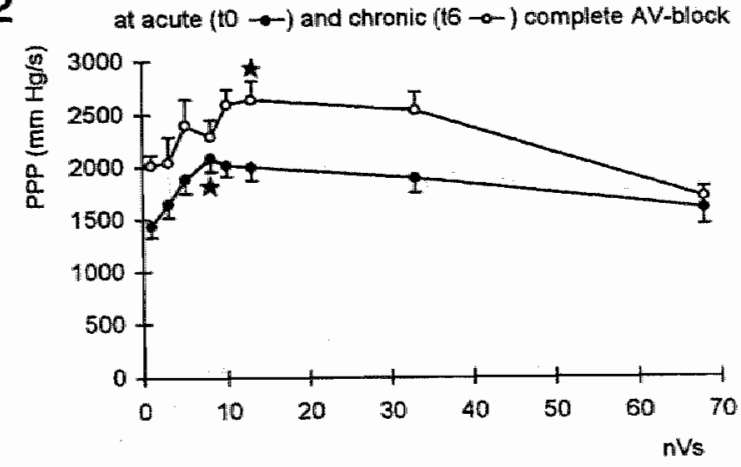

3 Postpacing Incidence of EBs in relation to the number of

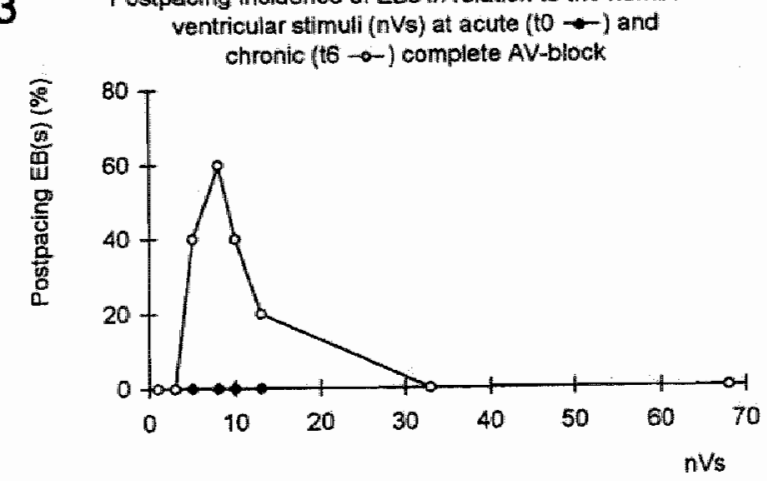


To ensure accuracy of our analysis and guarantee uniform interpretation of our results, a number of experiments were analyzed twice. One of these analyses was done by an independent observer without knowledge of the experimental circumstances.

\section{Additional data analysis in dogs used for fixed rate pacing}

In addition to the above described parameters, we determined the rate of decay of post-pacing-potentiation during idioventricular rhythm and fixed rated pacing, in the 5 dogs in whom fixed rate pacing was performed. This rate of decay of potentiation is thought to index the percent of calcium recycled through the sarcoplasmic reticulum, termed the recirculation fraction. ${ }^{17,19,33}$ After the initial post-pacing-potentiation, subsequent beats display a gradual geometric decline in contractility to baseline when all post-pacing intervals are of equal cycle length. To obtain the recirculation fraction, the magnitude of potentiation $\left(R_{n}\right)$ was determined for the first 6 beats post extrastimulation:

$$
\mathrm{R}_{\mathrm{n}}=\left[\mathrm{dP} / \mathrm{d} \mathrm{t}_{\max }(\mathrm{n}) / \mathrm{dP} / \mathrm{d} \mathrm{t}_{\max }(\mathrm{ss})\right]-1
$$

where the numerator and the denominator are the values of $d P / d t_{\max }$ for beat $n(n=1$ to 6) and the fully decayed state (steady state; ss), respectively. $R_{n}$ follows a geometric decay, that is, $R_{n}=\kappa^{n} * R_{1}$, were $\kappa$ is the recirculation fraction $(\kappa<1.0)$. It follows that $R_{n+1}=R_{n} * \kappa$, and applying linear regression to the $R_{n}$ versus the $R_{n+1}$ points yields the value for $\kappa .{ }^{17,19,33}$

\section{Heart Weight}

After a final experiment ( $56 \pm 25$ days after creation of atrioventricular-block, table 1), the heart weight of the dogs used in this study was determined and compared with previous observations, ${ }^{25,26}$ to confirm the presence of cardiac hypertrophy. After excision, the hearts were rinsed with water and the total heart weight was assessed and related to body weight. Six of the 17 hearts were stored in a formaldehyde solution for at least 2 weeks. In these hearts, the weight of the right and left ventricle (including septum) was determined and related to body weight (table 1).

\section{Statistics}

All data are presented as mean \pm standard deviation (SD), unless otherwise stated. To determine statistical significance, repeated measures analyses of variance (ANOVA) followed by Bonferroni's $t$-test, two-tailed Student's $t$-test for paired events (in case of 
two group comparison) and Chi-square testing were used. $\mathrm{P}$ values $<0.05$ were considered significant.

Table 2: Electrophysiological and hemodynamic adaptations induced by the creation of complete AV-block $(\mathrm{N}=10)$

\begin{tabular}{|c|c|c|c|}
\hline & Sinus Rhythm & $\begin{array}{l}\text { Acute AV-block } \\
\text { (t0) }\end{array}$ & $\begin{array}{l}\text { Chronic } \\
\text { AV-block (t6) }\end{array}$ \\
\hline $\mathrm{CL}$ & $527 \pm 70$ & $1250 \pm 365^{*}$ & $1538 \pm 330^{*}$ \\
\hline LV APD & $219 \pm 16$ & $287 \pm 18^{*}$ & $395 \pm 68 * n$ \\
\hline RV APD & $203 \pm 20$ & $256 \pm 18^{*}$ & $331 \pm 59 * "$ \\
\hline $\mathrm{Vs}-\mathrm{V}$ & $635 \pm 192$ & $1235 \pm 707^{*}$ & $1209 \pm 609^{*}$ \\
\hline LV ESP & $88 \pm 15$ & $90 \pm 12$ & $104 \pm 10$ \\
\hline LV EDP & $12 \pm 2$ & $15 \pm 3^{*}$ & $10 \pm 6^{\prime \prime}$ \\
\hline $\mathrm{LV} \mathrm{dP} / \mathrm{dt}$ & $1109 \pm 330$ & $992 \pm 284$ & $1726 \pm 332 * *$ \\
\hline PP LV dP/dt, 1 th beat & $1777 \pm 515^{\$}$ & $1865 \pm 459^{\$}$ & $2398 \pm 576^{* 11}$ \\
\hline $\mathrm{PP} L V \mathrm{dP} / \mathrm{dt}$, 2nd beat & $1498 \pm 592$ & $1563 \pm 452$ & $2183 \pm 585^{* \prime \prime}$ \\
\hline PP LV dP/dt, 3th beat & $1393 \pm 514$ & $1372 \pm 432$ & $1989 \pm 452^{* \prime \prime}$ \\
\hline Inducible dogs & & $0 \backslash 10$ & $6110^{*}$ \\
\hline $\mathrm{DAD}$ & & $0 \backslash 10$ & $8 \backslash 10^{*}$ \\
\hline VT & & $0 \backslash 10$ & $1 \backslash 10$ \\
\hline $\mathrm{nEB}$ & - & 0 & $12 \pm 23$ \\
\hline
\end{tabular}

AV-block: atrioventricular-block, CL: cycle length (ms), LV: left ventricle, RV: right ventricle, APD: action potential duration at $100 \%$ repolarization, Vs-V; first post-pacing interval, ESP: end systolic pressure, EDP: end diastolic pressure, DAD: delayed afterdepolarization, VT: ventricular tachycardia, $\mathrm{nEB}$ : mean number (n) of post-pacing induced ectopic beats per dog. All data mean $\pm \mathrm{SD}$ :*: $\mathrm{p}<0.05$, compared to sinus rhythm," $\mathrm{p}<0.05$, compared to acute AV-block, ${ }^{\$}: \mathrm{p}<0.05$, compared to pre-pacing $+\mathrm{LV} \mathrm{dP} / \mathrm{dt}$ 

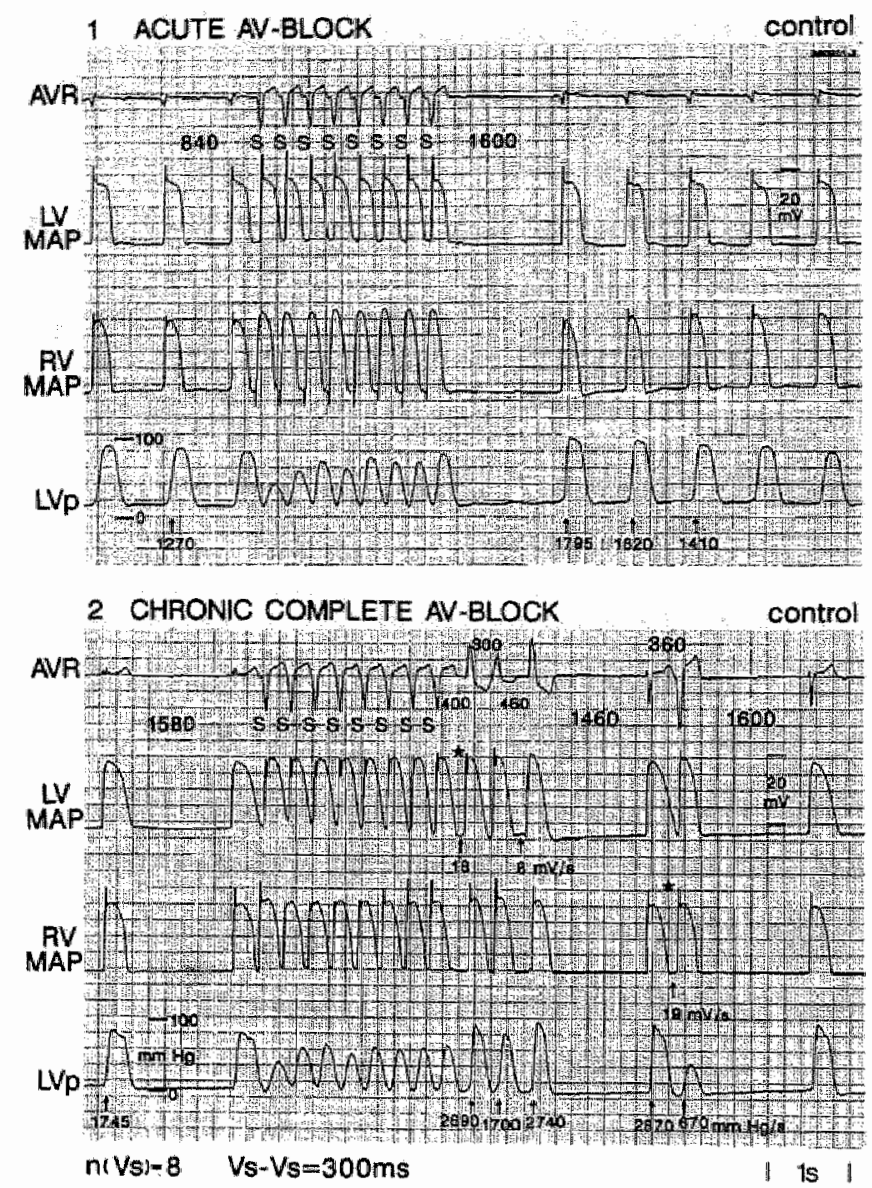

Figure 2: The effect of pacing during control circumstances at acute and chronic atrioventricular-block (AV-block) in the same dog.

In each panel ECG lead AVR, a left ventricular (LV) and right ventricular (RV) monophasic action potential (MAP) recording and the LV pressure curve ( $L V p)$ are shown.

In panel 1 , the idlioventricular thythm (cycle length $840 \mathrm{~ms}$ ) is interrupted by the start of a pacing train consisting of 8 stimuli (S) paced from the RV MAP electrode with an interstimulus interval (Vs-Vs) of $300 \mathrm{~ms}$. After this pacing train, no ectopic beats (EBs) are induced, nor are delayed afterdepolarizations (DADs) visualized in either of the MAPs. Before pacing, the $+\mathrm{LV} \mathrm{dP} / \mathrm{dt}$ equals $1270 \mathrm{~mm} \mathrm{Hg} / \mathrm{s}$, which clearly increases postpacing. In the following beats, the $+\mathrm{LV} \mathrm{dP} / \mathrm{dt}$ declines to prepacing values.

In the same dog, after 6 weeks of complete AV-block (panel 2), the idioventricular cycle length and LV and RV APD had prolonged The prepacing $+\mathrm{LV} \mathrm{dP} / \mathrm{dt}$ has increased to $1745 \mathrm{~mm} \mathrm{Hg} / \mathrm{s}$. At this time, the pacing protocol resulted in the induction of 3 EBs immediately postpacing. which coincide with the occumence of DADs in the LV MAP recording (indicated by the asterisk). Postpacing, the +LV dP/dt has increased to $2690 \mathrm{~mm} \mathrm{Hg} / \mathrm{s}$ which declines gradually. 


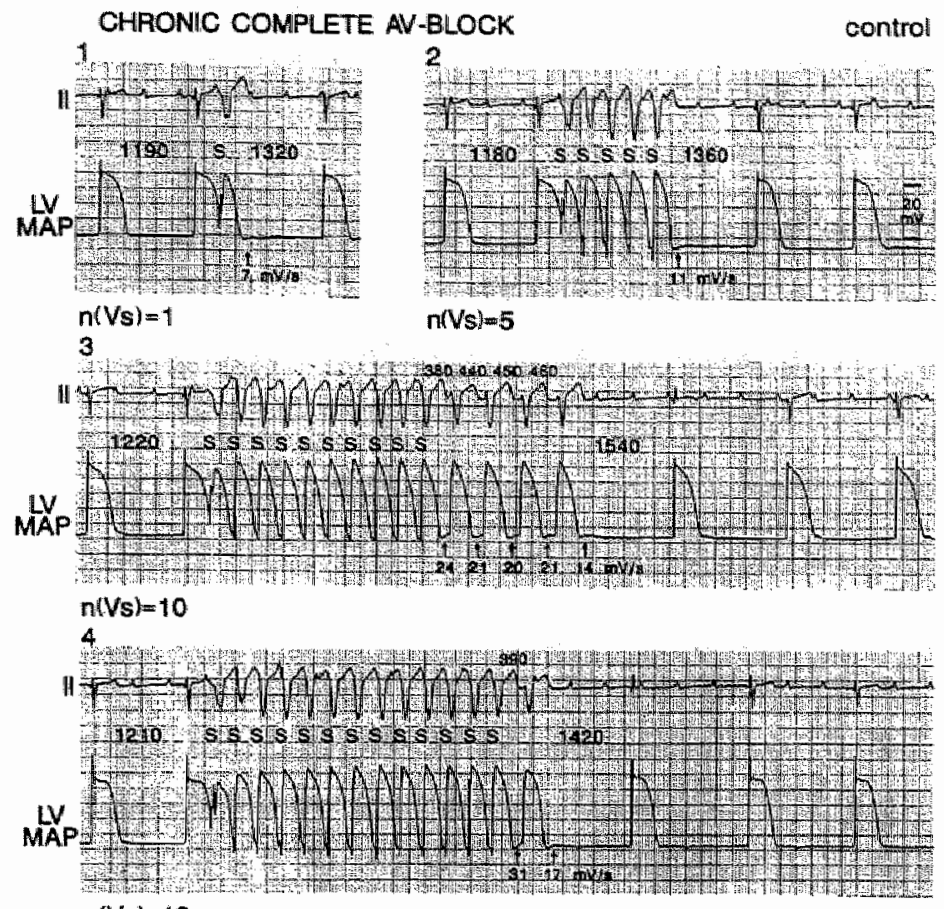
$n(V s)=13$

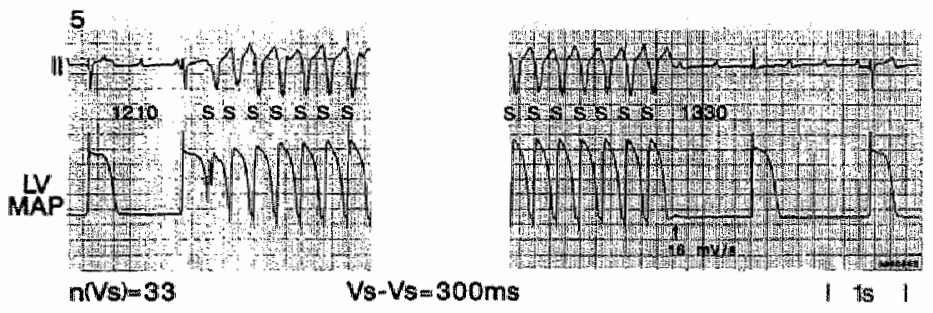

Figure 3: The effect of different durations of pacing on the induction of ectopic beats (EBs) in a specific dog.

In each panel ECG lead II and a left ventricular (LV) monophasic action potential (MAP) recording are shown. In panel 1 , the idioventricular rhythm (cycle length $1190 \mathrm{~ms}$ ) is interrupted by pacing with one stimulus (S) with an interstimulus interval (Vs-Vs) of $300 \mathrm{~ms}$. This is not followed by the occurrence of a delayed afterdepolarization (DAD) postpacing. The diastolic slope remains below $1.0 \mathrm{mV} / \mathrm{s}$ (arrow). In panel 2, pacing with 5 stimuli with an identical interstimulus interval results in a small DAD with a diastolic slope of $11 \mathrm{mV} / \mathrm{s}$. Further increasing the number of stimuli to 10 stimuli resulted in a higher slope of the DADs $(20 \mathrm{mV} / \mathrm{s}$ ) and in 4 triggered EBs post-pacing (panel 3). Increasing the number of stimuli to 13 (panel 4) and 33 stimuli (panel 5), results in a decreased induction of EBs and in a decreased slope of the DADs. 


\section{Results}

\section{Hypertrophy}

The heart weight at autopsy after $56 \pm 25$ days of complete atrioventricular-block, confirmed the presence of hypertrophy in all dogs studied, with a mean heart to body weight ratio of $10.9 \pm 1.5 \mathrm{~g} / \mathrm{kg}$ (range $9.1-13.9 \mathrm{~g} / \mathrm{kg}$, table 1). The subgroup analysis confirmed the involvement of both ventricles in the hypertrophy process with a left ventricle to body weight ratio of $5.8 \pm 1.2$ and a right ventricle to body weight ratio of $2.4 \pm 0.3 \mathrm{~g} / \mathrm{kg}$. Electrical and functional adaptations during spontaneous rhythm

During sinus rhythm, the ventricular cycle length was $527 \pm 70 \mathrm{~ms}$ (table 2). Directly after creation of complete atrioventricular-block the ventricular cycle length prolonged which was accompanied by an increase in both the left and right ventricular action potential duration (table 2, $\mathrm{p}<0.05$, for both). Both the left and right ventricular action potential duration prolonged further after 6 weeks of atrioventricular-block (table $2, p<0.05$, for both).

Hemodynamically, creation of complete atrioventricular-block was associated with a significant increase in left ventricular end diastolic pressure (from $12 \pm 2$ to $15 \pm 3 \mathrm{~mm} \mathrm{Hg}$, table $2, \mathrm{p}<0.05$ ), while the left ventricular end systolic pressure and $+\mathrm{LV} \mathrm{dP} / \mathrm{dt}$ remained similar to sinus rhythm values (table 2). At 6 weeks of chronic complete atrioventricular-block, the left ventricular end diastolic pressure had declined to $10 \pm 6 \mathrm{~mm} \mathrm{Hg} / \mathrm{s}$ (table $2, \mathrm{p}<0.05$ ), which was comparable to the sinus rhythm value. During chronic atrioventricular-block, the $+\mathrm{LV} \mathrm{dP/dt} \mathrm{had} \mathrm{increased} \mathrm{to} 1726 \pm 332$ as compared to $1109 \pm 330$ during sinus rhythm $992 \pm 284 \mathrm{~mm} \mathrm{Hg} / \mathrm{s}$ at acute atrioventricular-black ( $\mathrm{p}<0.05$, for both, table 2), indicating that the intrinsic contractility is increased.

\section{Post-pacing potentiation}

\section{General}

When all pacing protocols ( $\mathrm{n}=80,10$ dogs) were analyzed together, programmed electrical stimulation resulted in an increase of the first post-pacing $+\mathrm{LV} \mathrm{dP} / \mathrm{dt}$ during sinus rhythm, as well as during atrioventricular-block (table 2). By an comparable first post-pacing interval, the post-pacing $+\mathrm{LV} \mathrm{dP/dt} \mathrm{was} \mathrm{higher} \mathrm{at} \mathrm{chronic} \mathrm{atrioventricular-}$ block than at acute atrioventricular-block (2398 \pm 576 versus $1865 \pm 59 \mathrm{~mm} \mathrm{Hg} / \mathrm{s}$, table 2, $\mathrm{p}<0.05$ ). Within 3 beats, most of the post-pacing potentiation disappeared and the $+\mathrm{LV}$ $\mathrm{dP} / \mathrm{dt}$ returned to pre-pacing values (table 2). 


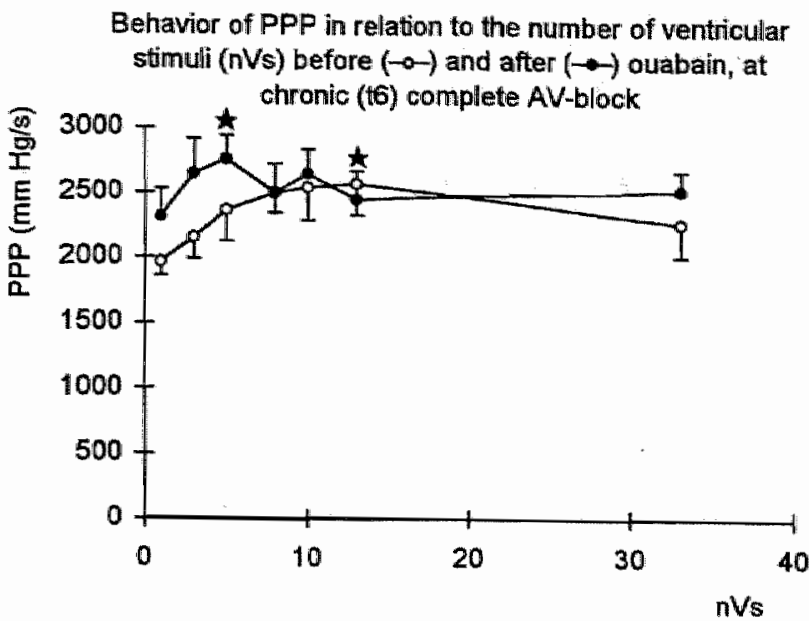

2

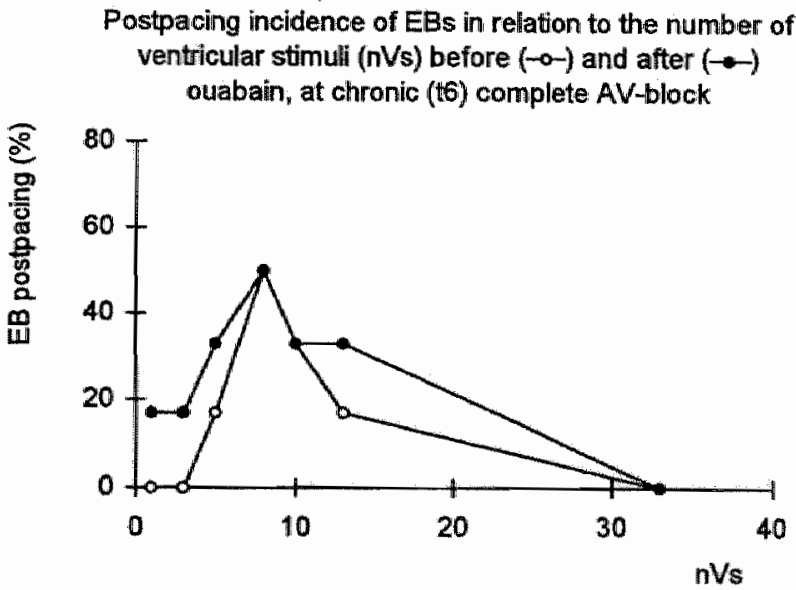

Figure 4: The effect of increasing the number of stimuli (nVs: interstimulus interval $300 \mathrm{~ms}$ ) on postpacing potentiation $(\mathrm{PPP}:+\mathrm{LV} \mathrm{dP} / \mathrm{dt}$ ) and inducibility of ectopic beats (EBs) at chronic complete AV-block, before and after ouabain administration.

In both panels on the horizontal axis $n \mathrm{Vs}$, in relation to the postpacing $+\mathrm{LV} \mathrm{dP} / \mathrm{dt}$ (panel 1, mean $\pm \mathrm{SEM}$ ) or the percentage of pacing trains that were followed by the occurrence of $\geq 1 \mathrm{~EB}$ (panel 2) on the vertical axis, before and after ouabain. Data are represented as mean $\pm S E M, *: p<0.05$, compared to $n V s=1$

Before ouabain administration, the initial increase in $\mathrm{nVs}$ is accompanied by an increase in postpacing $+L V d P / d t$. After ouabain, the amount of postpacing $+L V d P / d t$ is higher (panell). This is accompanied by a broader ranch over which EBs can be induced (panel 2). 
1 CHRONIC COMPLETE AV-BLOCK control
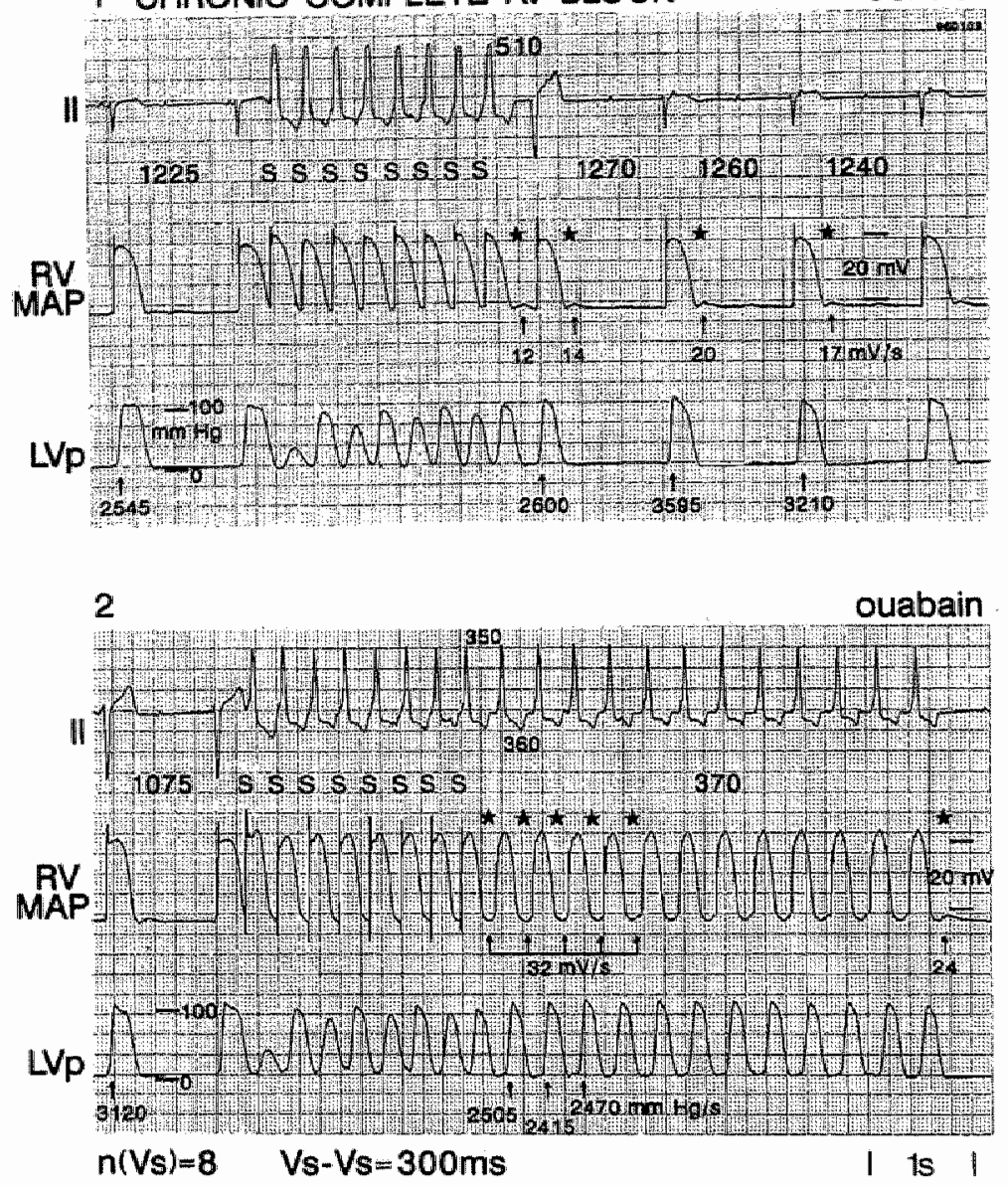

Figure 5: Illustration of the effect of ouabain on the inducibility of ectopic beats (EBs) at chronic complete AV-block in an individual dog.

In each panel, ECG lead II, a right ventricular (RV) monophasic action potential (MAP) recording and the left ventricular pressure ( $L V p)$ curve are shown.

In panel 1, under control conditions, the idioventricular rhythm (cycle length $1225 \mathrm{~ms}$ ) is interrupted by the start of a pacing train, consisting of 8 stimuli ( $S$, interstimulus interval ( $\mathrm{Vs}-\mathrm{V}_{\mathrm{s}}$ ) of $300 \mathrm{~ms}$ ). This is followed by one delayed afterdepolarization (DAD) related EB. The DADs are indicated by an asterisk, while their slope is illustrated below the RV MAP with arrows. The $+\mathrm{LV} \mathrm{dP} / \mathrm{dt}$ is increased after pacing: especially the second beat shows a marked increase. Both the DADs and the postpacing $+\mathrm{LV} d \mathrm{~d} / \mathrm{dt}$ diecline during the following beats.

After ouabain, the prepacing $+\mathrm{LV} \mathrm{dP/dt} \mathrm{is} \mathrm{higher} \mathrm{than} \mathrm{during} \mathrm{baseline} \mathrm{circumstances} \mathrm{(panel} \mathrm{2).} \mathrm{The} \mathrm{same}$ pacing train results in the occurrence of a VT consisting of 12 beats. During this VT, the DADs are visible as a steep upgoing slope in the MAP. 


\section{Effects of the variable number of stimuli}

During SR the amount of increase in post-pacing $+\mathrm{LV} \mathrm{dP} / \mathrm{dt}$ depended strongly on the number of stimuli and demonstrated a biphasic response (figure 1, panel 1). Initially, a concordant relation is present between the increase in number of stimuli and increase in post-pacing-potentiation, showing a maximal value after 5-13 stimuli (figure 1, panel 1). This biphasic potentiation response post-pacing was similar during acute and chronic atrioventricular-block (figure 1, panel 2). However, the curve at chronic atrioventricular-block was shifted to a higher $+\mathrm{LV} \mathrm{dP/dt}$ (figure 1, panel 2, $\mathrm{p}<0.05$ ).

\section{Triggered ventricular arrhythmias}

\section{General}

Following acute atrioventricular-block, programmed electrical stimulation never resulted in the induction of any ectopic beats, nor in the of delayed afterdepolarizations in the monophasic action potentials (table 2 and figure 2, panel 1). In contrast, 6 weeks later, the same stimulation protocol resulted in the induction of single or multiple ectopic beats in $6 / 10$ dogs and in VT in $1 / 10$ dogs (table 2, $p<0.05$ for inducibility). The occurrence of ectopic beats coincided with the visualization of delayed afterdepolarizations in the left ventricular and/or right ventricular monophasic action potential recordings in $8 / 10$ dogs (table $2, \mathrm{p}<0.05$ ). For 1 individual dog, the effect of pacing at acute and chronic atrioventricular-block is illustrated in figure 2 .

\section{Effects of the variable number of stimuli}

Like the amount of post-pacing-potentiation, also the inducibility of ectopic beats varied with the duration of pacing (figure 3). As can be seen in figure 1 (panel 3), most ectopic beats were induced after 5-13 stimuli. As illustrated in panel 2 of figure 3, this corresponded with the highest post-pacing $+\mathrm{LV} \mathrm{dP} / \mathrm{dt}$.

\section{Interventions}

\section{Ouabain}

Both at acute and chronic atrioventricular-block, the idioventricular cycle length, left and right ventricular action potential duration, the first post-pacing interval and left ventricular end diastolic pressure remained similar after the administration of ouabain, while the left ventricular end systolic pressure and pre- and post-pacing $+\mathrm{LV} \mathrm{dP/dt}$ 
increased (table 3). At both times, the biphasic relation between post-pacing $+\mathrm{LV} \mathrm{dP/dt}$ and the number of stimuli was not affected by the low dose of ouabain.

Table 3: Effect of ouabain $(20 \mu \mathrm{g} / \mathrm{kg})$ on baseline characteristics

\begin{tabular}{lllll}
\hline & \multicolumn{2}{c}{ Acute AVB (A) } & Chronic AVB & (B) \\
\hline & Control & Ouabain & Control & Ouabain \\
CL & $1437 \pm 354$ & $1563 \pm 368$ & $1586 \pm 284$ & $1506 \pm 355$ \\
LV APD & $266 \pm 16$ & $276 \pm 38$ & $381 \pm 75$ & $356 \pm 59$ \\
RV APD & $256 \pm 33$ & $251 \pm 18$ & $335 \pm 42$ & $316 \pm 42$ \\
Vs-V & $1447 \pm 887$ & $1460 \pm 496$ & $1436 \pm 557$ & $1484 \pm 950$ \\
& & & & \\
LV ESP & $83 \pm 12$ & $88 \pm 13^{*}$ & $102 \pm 10$ & $108 \pm 10^{*}$ \\
LV EDP & $15 \pm 4$ & $15 \pm 4$ & $8 \pm 5$ & $7 \pm 3$ \\
LV dP/dt & $823 \pm 232$ & $1004 \pm 346^{*}$ & $1686 \pm 285$ & $2075 \pm 534^{*}$ \\
PP +LV dP/dt, & $1674 \pm 252^{\$}$ & $1793 \pm 311^{*} \$$ & $2340 \pm 224^{\$}$ & $2557 \pm 146^{*} \$$ \\
1 th beat & & & & \\
& & & 316 & 416 \\
Inducible dogs & $0 \backslash 6$ & 016 & 416 & 516 \\
DAD & 016 & 016 & 016 & 116 \\
VT & $0 \backslash 6$ & 016 & $3 \pm 1$ & $9 \pm 10$ \\
nEB & 0 & 0 & & \\
\hline
\end{tabular}

AVB: atrioventricular-block, CL: cycle length (ms), LV: left ventricle, RV: right ventricle, APD: action potential duration at $100 \%$ repolarization, $V_{s-V}$; first post-pacing interval, ESP: end systolic pressure, EDP: end diastolic pressure, DAD: delayed afterdepolarization, VT: ventricular tachycardia, nEB: number $(\mathrm{n})$ of post-pacing induced ectopic beats per dog. All data mean $\pm \mathrm{SD}$ *: $\mathrm{p}<0.05$, compared to control, $5: \mathrm{p}<0.05$, compared to pre-pacing $+\mathrm{LV} \mathrm{dP} / \mathrm{dt}$ 

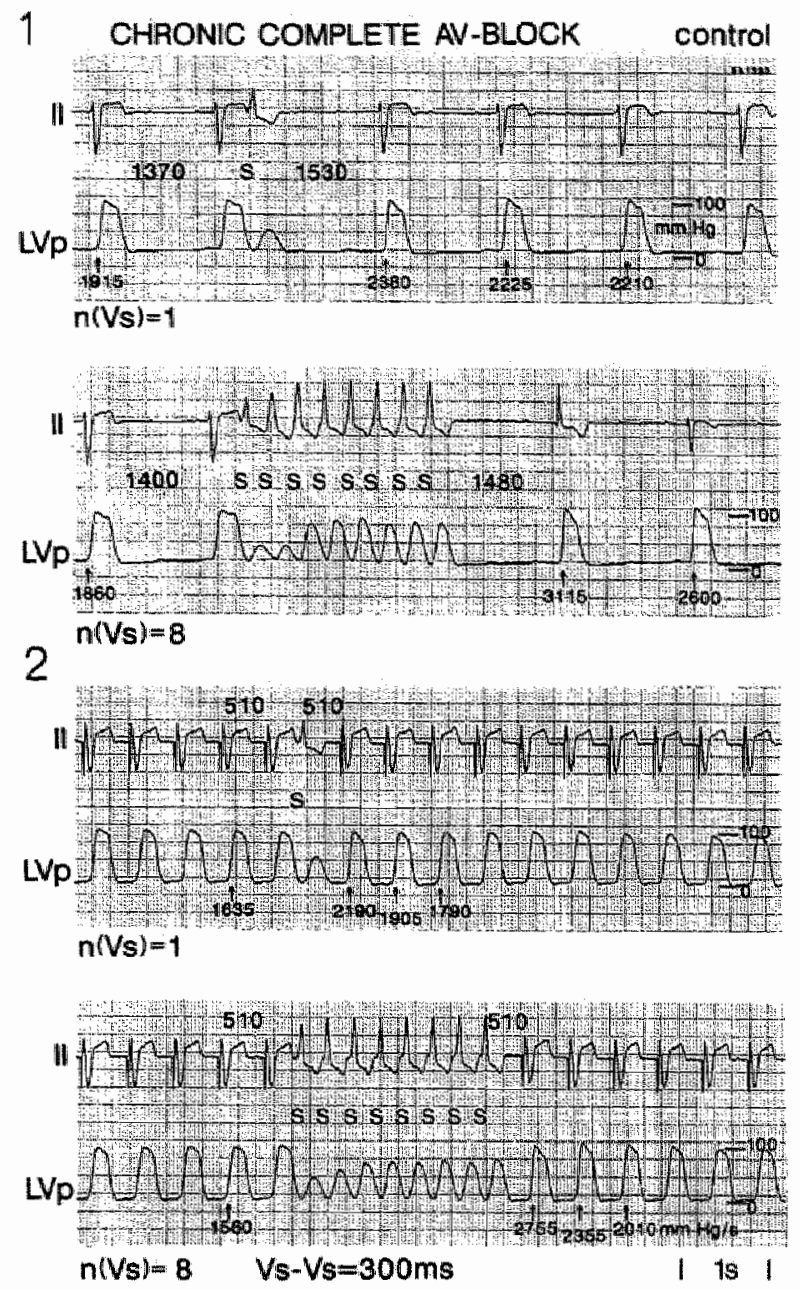

Figure 6: Controlling the pre- and postpacing intervals on postpacing potentiation ( $P P P:+L V d P / d t)$. In each panel ECG lead II and the left ventricular pressure $\left(L V_{p}\right)$ curve is depicted.

In panel 1 the effect of pacing with 1 and 8 extrastimuli ( $\mathrm{S}$, interval of $300 \mathrm{~ms}$ ) an postpacing $+\mathrm{LV} \mathrm{dP} / \mathrm{dt}$ is illustrated. The postpacing $+\mathrm{LV} \mathrm{dP} / \mathrm{dt}$ is higher after 8 than after 1 extrastimulus. However, the pre- and postpacing interval and focus are different.

In panel 2, a similar effect of pacing on the postpacing $+\mathrm{LV} \mathrm{dP} / \mathrm{dt}$ is visible, when the same pacing trains are repeated during fixed rate pacing from the epicardial electrode with an interval of $510 \mathrm{~ms}$ (equal to the sinus rhythm frequency). As in panel $\mathrm{l}$, the postpacing $+\mathrm{LV} \mathrm{dP/dt}$ increases when the number of extrastimuli is increased from 1 to 8 .

Additionally, this figure illustrates that increasing the heart rate by ventricular pacing results in a decrease in pre- and postpacing $+\mathrm{LV}$ dP/dt at 6 weeks of complete atrioventricular-block 

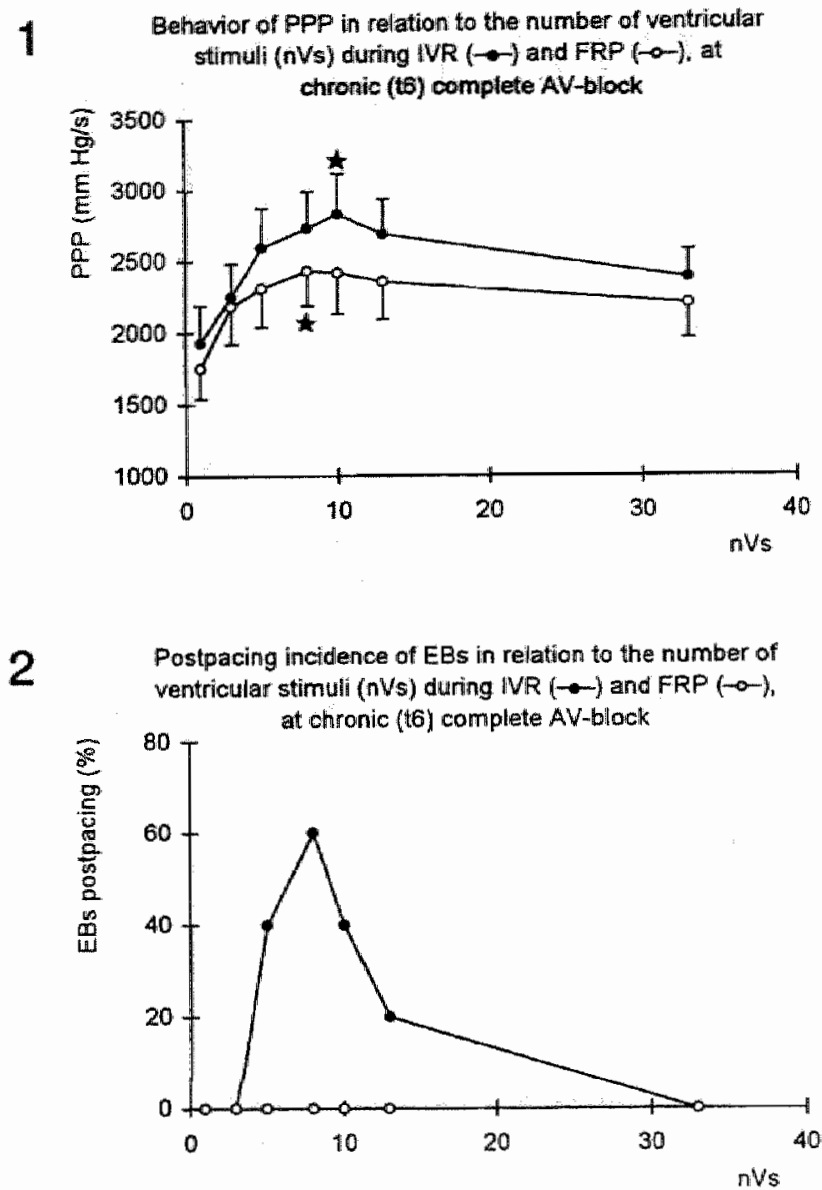

Figure 7: The effect of fixed rate ventricular (FRP) pacing at a sinus rhythm frequency on postpacing potentiation (PPP: $+\mathrm{LV} \mathrm{dP} / \mathrm{dt}$ ) and inducibility of ectopic beats (EBs) at 6 weeks of complete atrioventricular-block (AV-block).

In both panels the number of ventricular stimuli ( $\mathrm{nVs}$, interstimulus interval $300 \mathrm{~ms}$ ) on the horizontal axis, in relation to the postpacing $+\mathrm{LV} \mathrm{dP/dt}$ (panel 1 , mean $\pm \mathrm{SEM}$ ) or the percentage of pacing trains that was followed by $\geq \mathbb{E B}$ (panel 2) on the vertical axis, during idioventricular rhythm (TVR) and during FRP at $505 \pm 49 \mathrm{~ms}$ (equal to pre-existent sinus rhythm). Data are presented as mean $\pm S E M_{,}^{*} * \mathrm{p}^{*}<0.05$, compared to $n \mathrm{~V} s=1$.

At both times, the initial increase in stimuli is accompanied by an increase in postpacing $+\mathrm{LV} d \mathrm{~d} / \mathrm{dt}$. However, during IVR the amount of postpacing $+\mathrm{LV} d \mathrm{dP} / \mathrm{dt}$ is significantly higher than during FRP (pane) $1)$. The decrease in postpacing $+\mathrm{LV} \mathrm{dP/dt}$ during FRP is accompanied by complete suppression of pacing induced EBs. 
Table 4: Effect of fixed rate pacing (FRP) on hemodynamics, absolute and relative post-pacing potentiation and recirculation fraction

\section{Acute AVB (A)}

TVR

\section{ESP}

LV EDP

$\mathrm{LV} \mathrm{dP} / \mathrm{dt}$

$95 \pm 12$

$16 \pm 3$

$914 \pm 222$

$1713 \pm 343$

$\mathrm{PP}+\mathrm{LV} \mathrm{dP} / \mathrm{dt}$,

1th beat

$$
\kappa
$$$$
\mathrm{R}_{\mathrm{n}}
$$

\section{FRP}

$98 \pm 14$

$9 \pm 4 *$

$1096 \pm 386$

$1900 \pm 516^{*}$

$0.81 \pm 0.21$

$0.66 \pm 0.10$
Chronic AVB

IVR

$98 \pm 14$

$9 \pm 5$

$1680 \pm 428$

$2368 \pm 502$

$0.45 \pm 0.21^{\$}$

$0.66 \pm 0.10$

\section{FRP}

$99 \pm 17$

$6 \pm 3^{*}$

$1343 \pm 323 *$

$2172 \pm 502$

$0.59 \pm 0.12$

$0.62 \pm 0.09$

\begin{tabular}{lllll} 
Inducible dogs & $0 / 5$ & $0 / 5$ & $4 / 5$ & $0 / 5$ \\
DAD & $0 / 5$ & $0 / 5$ & $4 / 5$ & $0 / 5$ \\
VT & $0 / 5$ & $0 / 5$ & $0 / 5$ & $0 / 5$ \\
nEB & 0 & 0 & $3 \pm 1$ & 0 \\
\hline
\end{tabular}

AVB: atrioventricular-block, IVR: idioventricular rhythm, FRP: fixed rate pacing, LV ESP: left ventricular end-systolic pressure, LV EDP: left ventricular end-diastolic pressure, $\mathbb{R}_{n}$ : relative potentiation, $\kappa:$ recirculation fraction, DAD: delayed afterdepolarization, VT: ventricular tachycardia, nEB: mean number ( $\mathrm{n}$ ) of post-pacing induced ectopic beats per dog. Fixed rate pacing was performed as an intervall of $520 \pm 40$ ms. All data mean $\pm \mathrm{SD},{ }^{*}: \mathrm{p}<0.05$, compared to IVR at the same time (acute or chronic atrioventricular-block), $\$$ : $<<0.05$ compared to IVR at acute atriowentricular-block.

Although the pre- and post-pacing $+\mathrm{LV} \mathrm{dP} / \mathrm{dt}$ increased after ouabain at acute atrioventricular-block, they remained lower than the baseline $+\mathrm{LV} \mathrm{dP/dt}$ at 6 weeks (table 3). This increase in pre-and post-pacing $+\mathrm{LV} \mathrm{dP} / \mathrm{dt}$ was not accompanied by the occurrence of ectopic beats.

In contrast, increasing the pre- and post-pacing $+\mathrm{LV} \mathrm{dP/dt}$ at chronic atrioventricularblock with ouabain (from $2340 \pm 224$ to $2557 \pm 146 \mathrm{~mm} \mathrm{Hg} / \mathrm{s}, \mathrm{p}<0.05$, table 3 (side B), figure 4 , panel 1) resulted in 1) an increased frequency of ectopic beat occurrence $(p<0.05$, figure 4 , panel 2) and 2) an increased number of induced ectopic beats (table 3 , 

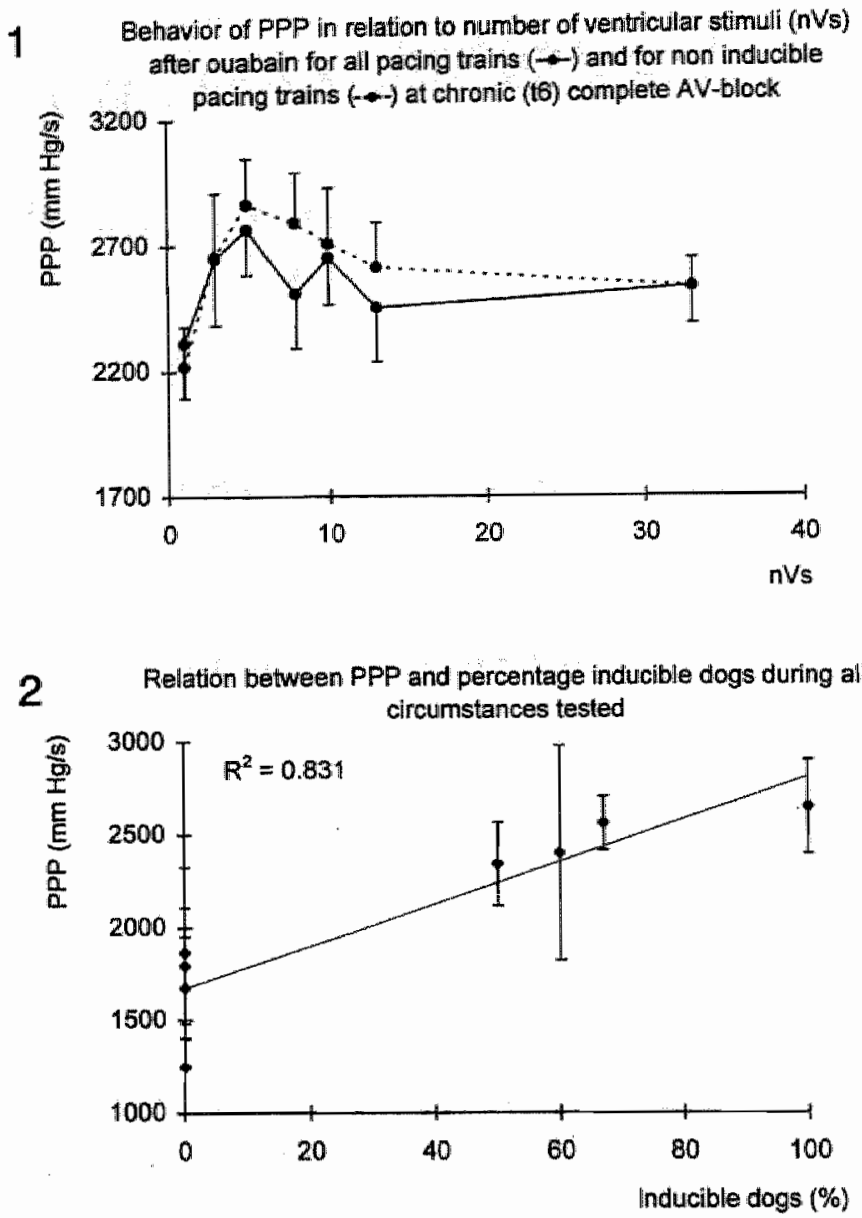

Figure 8: In panel 1, the relation between ectopic beats (EBs) and the degree of postpacing potentiation (PPP: $+\mathrm{LV} \mathrm{dP/dt}$ ) and in panel 2 the relation between the amount of PPP and inducibility of EBs.

In panel 1, the effect of EBs on the degree of PPP is illustrated for the curve before and after ouabain at 6 weeks of atrioventricular-block. This curve was previously shown in figure 4 . From this figure it becomes clear that PPP is very irregular after ouabain (solid line). The dotted line indicates the PPP curve after ouabain, when all pacing trains that were followed by EBs were excluded. This resulted in a more smoothened, and higher PPP curve, which suggests that the EBs are responsible for the irregularities. In panel 2 , the inducibility of EBs for each group of dogs is related to the postpacing $+\mathrm{LV} \mathrm{dP} / \mathrm{dt}$ of those dogs (mean $\pm \mathrm{SD}$ ). This figure illustrates that the post-pacing $+\mathrm{LV}$ dP/dt shows a linear relation to inducibility. When postpacing $+\mathrm{LV} \mathrm{dP} / \mathrm{dt}$ is low, the chances of inducing EBs by pacing are low. In contrast, when the postpacing $+\mathrm{LV} \mathrm{dP} / \mathrm{dt}$ is high, all dogs becone inducible. 
side B). Due to the limited number of dogs studied, this increase in number of ectopic beats induced did not reach statistical significance. However, the importance of this change for an individual dog is illustrated in figure 5. Without ouabain, pacing only results in the induction of a single ectopic beat, while after a low dose ouabain the same pacing protocol results in the occurrence of VT.Fixed rate pacing

At acute atrioventricular-block, continuous pacing at a fixed rate of $520 \pm 40 \mathrm{~ms}$ (similar to the sinus rhythm interval), reduced the left ventricular end diastolic pressure and had no effect on left ventricular end systolic pressure and $+\mathrm{LV} \mathrm{dP/dt} \mathrm{(table} \mathrm{4).} \mathrm{In} \mathrm{contrast,}$ fixed rate pacing at chronic atrioventricular-block, resulted in a decrease of the $+\mathrm{LV}$ $\mathrm{dP} / \mathrm{dt}$ (from $1680 \pm 428$ to $1343 \pm 323, \mathrm{p}<0.05$ ), while both left ventricular end systolic pressure and end diastolic pressure pressures remained similar (left ventricular end systolic pressure idioventricular thythm: $98 \pm 14$ versus $99 \pm 17 \mathrm{~mm} \mathrm{Hg}$ during fixed rate pacing, left ventricular end diastolic pressure idioventricular rhythm: $9 \pm 5$ versus $6 \pm 3$ $\mathrm{mm} \mathrm{Hg}$ during fixed rate pacing, table 4). The initial increase in post-pacing $+\mathrm{LV} \mathrm{dP/dt}$ on increasing the number of stimuli that was visible during idioventricular thythm, remained present during fixed rate left ventricular epicardial pacing (figure 6). This results in an identical shape of the post-pacing $+\mathrm{LV} \mathrm{dP/dt} \mathrm{curve} \mathrm{during} \mathrm{fixed} \mathrm{rate} \mathrm{pacing,}$ although the curve was overall lower (figure 7, panel 1, $<<0.05$ compared to idioventricular rhythm). This was accompanied by the inability to induce delayed afterdepolarizations and triggered ectopic beats (figure 7, panel 2). Although, the post-pacing-potentiation curve at 6 weeks of atrioventricular-block declined during fixed rate pacing, it remained higher than the acute atrioventricular-block fixed rate pacing and idioventricular rhythm curves $(\mathrm{p}<0.05$ ). Although absolute post-pacing-potentiation was higher at chronic atrioventricular-block, both during idioventricular rhythm and fixed rate pacing, there were also differences in the pre-pacing $+\mathrm{LV} \mathrm{dP/dt}$ (table 4). Correcting for this difference resulted in a higher relative post-pacing-potentiation at acute atrioventricular-block in comparison to chronic atrioventricular-block, both during idioventricular rhythm and fixed rate pacing (table 4). However, the decline of potentiation was similar during all circumstances, resulting in equal recirculation fractions ( $\kappa$, table 4).

\section{Ryanodine}

After ryanodine, the cycle length of the idioventricular rhythm shortened from $1315 \pm 220$ to $1034 \pm 200 \mathrm{~ms}(\mathrm{p}<0.05)$, which was accompanied by a shortening of both the left ventricular action potential duration from $404 \pm 67$ to $322 \pm 64 \mathrm{~ms}$ and right ventricular 
action potential duration from $323 \pm 44$ to $298 \pm 47 \mathrm{~ms}$ ( $\mathrm{p}<0.05$, for both, table 5). Ryanodine severely depressed cardiac contractile function. left ventricular end systolic pressure and pre- and post-pacing $+\mathrm{LV} \mathrm{dP} / \mathrm{dt}$ decreased, while the left ventricular end diastolic pressure increased ( $p<0.05$, for all, table 5): The biphasic response was maintained, while the negative inotropic action of the drug was accompanied by total suppression of ectopic beats and delayed afterdepolarizations (table 5).

Table 5: Effect of ryanodine on hemodynamic and electrophysiological parameters

\begin{tabular}{lll}
\hline & Control & Ryanodine \\
\hline CL & $1315 \pm 220$ & $1034 \pm 200^{*}$ \\
LV APD & $404 \pm 67$ & $322 \pm 64^{*}$ \\
RV APD & $323 \pm 44$ & $298 \pm 47^{*}$ \\
Vs-V & $1363 \pm 285$ & $968 \pm 194^{*}$ \\
& & \\
LV ESP & $116 \pm 9$ & $85 \pm 14^{*}$ \\
LV EDP & $11 \pm 5$ & $17 \pm 5^{*}$ \\
LV dP/dt & $2327 \pm 618$ & $759 \pm 185^{*}$ \\
PP LV dP/dt & $2643 \pm 252^{\$}$ & $1247 \pm 249^{*} \$$ \\
Inducible dogs & & \\
DAD & $4 / 4$ & $0 / 4^{*}$ \\
VT & $3 / 4$ & $0 / 4$ \\
nEB & $0 / 4$ & $0 / 4$ \\
\hline
\end{tabular}

CL: cycle length (ms), LV: left ventricle, RV: right ventricle, APD: action potential duration at $100 \%$ repolarization, Vs-V; first post-pacing interval, ESP: end-systolic pressure, EDP: end-diastolic pressure, DAD: delayed afterdepolarization, VT: ventricular tachycardia, $\mathrm{nEB}$ : mean number (n) of induced ectopic beats (EB) post-pacing per dog. All data mean \pm SD.

*: $\mathrm{p}<0.05$, compared to control, $\$:<\mathrm{p} 0.05$, compared to pre-pacing $+\mathrm{LV} \mathrm{dP/dt}$ 


\section{Relation between inotropic state and the occurrence of triggered arrhythmias}

To describe the post-pacing-potentiation curve, we determined the post-pacing $+\mathrm{LV}$ $\mathrm{dP} / \mathrm{dt}$ of any first post-pacing beat, independent of their idioventricular, ectopic or paced origin. Because there could be a different relation between ectopic beats and post-pacing-potentiation in comparison to normal idioventricular beats and post-pacing-potentiation, we analyzed the pacing trains on the basis of inducibility. The pacing trains not leading to ectopic beats showed a higher post-pacing-potentiation, explaining the saw tooth appearance. In figure 8, panel 1, this is illustrated for the post-pacing-potentiation curve after ouabain administration at 6 weeks of complete atrioventricular-block. Although less outspoken, the baseline post-pacing-potentiation curves at chronic atrioventricular-block were similarly affected.

Because both post-pacing-potentiation and induction of ectopic beats behaved similariy in response to pacing, we also investigated the relation between post-pacing-potentiation and inducibility. This linear relation $\left(\mathrm{R}^{2}=0.83\right)$ is illustrated in figure 8 , panel 2. When post-pacing-potentiation is low, the chances to induce ectopic beats in any of the dogs is low, while when post-pacing-potentiation is high, these chances are increased.

\section{Discussion}

The results of the present study indicate that steady state cardiac contractile function at 6 weeks of chronic atrioventricular-block is enhanced, which when challenged (potentiation) predisposes the heart for the development of delayed afterdepolarization dependent triggered ventricular arrhythmias.

\section{Structural adaptations}

In this study, we confirm that chronic complete atrioventricular-block, results in cardiac hypertrophy. ${ }^{9,12,13,25,26}$ The heart to body weight ratio found in the present study is higher than the body weight ratio of non hypertrophied dog hearts $5,12,25,26,34$ and similar to those previously reported in this model. ${ }^{9,12,13,25,26}$ The left and right ventricular to body weight ratios found, confirm the involvement of both ventricles in the hypertrophy process and are similar to those previously reported in this ${ }^{12,25,26}$ and other canine hypertrophy models. ${ }^{5,10,34,35}$ 


\section{Functional adaptations after the creation of complete atrioventricular-block}

\section{Preservation of left ventricular function during idioventricular rhythm}

In accordance with previous observations, we found an increase in left ventricular end diastolic pressure after the creation of atrioventricular-block. ${ }^{12,13}$ In canine chronic complete atrioventricular-block, some studies have reported spontaneous or exercise induced development of congestive heart failure, ${ }^{11,13}$ while others described the absence of heart failure in a follow up period from 2 weeks to 3 years of atrioventricularblock. ${ }^{9,12,24}$ In our experience, chronic complete atrioventricular-block in dogs only seldom results in overt congestive heart failure. This observation is supported by our finding of a normal left ventricular end diastolic pressure at 6 weeks of atrioventricularblock. Additional support for a, at least, maintained ventricular function at 6 weeks of atrioventricular-block, is suggested by the marked increase in $+\mathrm{LV} \mathrm{dP/dt}$ at decreased left ventricular end diastolic pressure and similar left ventricular end systolic pressure. This is a remarkable difference in comparison to the observations done in canine models of congestive heart failure, were a depressed $+\mathrm{LV} \mathrm{dP} / \mathrm{dt}$ is consistently found despite increases in preload. ${ }^{18,36,37}$ Although an increased contractile function can be suggested from the increased $+\mathrm{LV} \mathrm{dP} / \mathrm{dt}$, this has to be interpreted with caution since we did not study alterations in diastolic volume or pressure volume relations.

\section{Potentiation}

In the normal myocardium, potentiation of contraction following a premature extrasystole, is a well known phenomenon. ${ }^{17-19,33,38-40}$ It has been described that the degree of potentiation increases with shortening of the coupling interval of the extrasystole. ${ }^{18,33,38,39}$ Additionally, in the rat heart, a relation between the degree of post-pacing-potentiation and the number of interposed extrastimuli has been suggested. $^{39}$

We studied the effect of an increasing number of stimuli on post-pacing $+\mathrm{LV} \mathrm{dP/dt}$ in the intact dog heart during sinus rhythm and atrioventricular-block. At both time points, post-pacing potentiation was clearly present. Additionally, our data demonstrates that post-pacing-potentiation has a duration dependency: with an initial increase on increasing the number of stimuli to \pm 13 stimuli, followed by attenuation of post-pacing-potentiation with further increasing the number of stimuli (biphasic response). To our knowledge, we are the first to describe such an phenomenon in vivo. This response was not unique for the acute or chronic atrioventricular-block situation, but was also present 
during sinus rhythm. We excluded alterations in ventricular filling secondary to variations in the post-pacing interval as the cause for the initial increase in post-pacing potentiation of contraction, by repeating the extrastimulation during fixed rate pacing. Since the biphasic pattern of post-pacing-potentiation remained similar during fixed rate pacing, it can be suggested that this pattern is intrinsic to the myocardium.

Absolute post-pacing-potentiation is highest at chronic complete atrioventricular-block when the pacing trains are performed during idioventricular rhythm. However, at acute and chronic complete atrioventricular-block, there were differences in starting level, as indicated by the higher pre-pacing $+\mathrm{LV} \mathrm{dP} / \mathrm{dt}$ in the dog with chronic complete atrioventricular-block. When calculating the relative potentiation (table 4), it is clear that there is less potentiation at chronic complete atrioventricular-block, suggestive for less contractile reserve. However, when evaluating the recirculation fraction as indicator for differences in decline of potentiation between hypertrophied (chronic atrioventricularblock) and non-hypertrophied (acute atrioventricular-block) hearts, no differences were found. These observations are in contrast with previous observations on hypertrophied and failing hearts, were an enhanced relative increase in extrasystolic potentiation and a more rapid decay of potentiation were found. ${ }^{17,19,33}$ Since these alterations have been ascribed to abnormalities in calcium transients and calcium cycling, additional studies will be needed to evaluate calcium homeostasis.

\section{Electrophysiological adaptations}

\section{Action potential duration}

At acute atrioventricular-block, both the left and right ventricular action potential duration was prolonged, presumably due to the dramatic decrease in heart rate (table 2). In time, the action potential durations increase further, possibly exceeding the expected lengthening based on further slowing of the heart rate. This lengthening might be related to the structural adaptations (biventricular hypertrophy) that take place. This synergistic effect can explain our marked increase in action potential duration, which exceeds those reported previously in dogs with left ventricular hypertrophy. ${ }^{34}$

\section{Triggered ventricular arrhythmias}

The pacing protocol resulted not only in potentiation of contraction, but also provoked delayed afterdepolarizations and ventricular triggered arrhythmias. ${ }^{29,41}$ In the present study, we were able to induce ectopic beats with pacing in the majority of dogs at chronic atrioventricular-block, while at acute atrioventricular-block, no arrhythmias could be 
induced. The occurrence of ectopic beats coincided with the registration of delayed afterdepolarizations with the monophasic action potential, thereby suggesting delayed afterdepolarizations as the underlying mechanism. We have previously reported the induction of delayed afterdepolarization related ectopic beats after short-lasting pacing trains with short interstimulus intervals in conscious dogs with chronic complete atrioventricular-block. ${ }^{42}$ As in the present study, longer duration of pacing resulted in the suppression of ectopic beats and delayed afterdepolarizations. ${ }^{42}$

An increased propensity of hypertrophied myocardium to delayed afterdepolarization dependent triggered activity, has been previously described in vitro, but to our knowledge not in vivo. ${ }^{22,43-46}$ In these in vitro studies, the incidence of delayed afterdepolarizations and triggered activity with stimulation alone was rather low. Since our study was performed in intact animals, activity of the autonomic nervous system might have caused the higher incidence of delayed afterdepolarizations and related ectopic beats. This is supported by the in vitro studies, where the hypertrophied tissue appeared more susceptible to the development of delayed afterdepolarization dependent triggered activity after adrenergic stimulation. ${ }^{4-46}$

\section{Relation between challenged inotropic state, delayed afterdepolarizations and triggered arthythmias}

Remarkably, both past-pacing-potentiation and the inducibility of ectopic beats relate similarly to increasing the number of stimuli, resulting in a maximal post-pacing-potentiation and a maximal inducibility after 5-13 stimuli. However, ectopic beats are only induced at 6 weeks of complete atrioventricular-block, when post-pacing-potentiation is highest. This suggests the existence of a relation between the degree of post-pacing-potentiation and the occurrence of delayed afterdepolarizations and triggered ectopic beats. Since delayed afterdepolarizations specifically occur in the setting of intra-cellular calcium overload, ${ }^{47-49}$ the common etiology between the increased inotropic state (increased pre- and post-pacing $+\mathrm{LV} \mathrm{dP} / \mathrm{dt}$ ) and the increased sensitivity to delayed afterdepolarizations in chronic atrioventricular-block could relate to altered intracellular calcium handling. From the biphasic response of both post-pacing-potentiation and inducibility of ectopic beats, it is suggested that pacing results in dynamic alterations in intracellular calcium loading. At chronic atrioventricular-block, these dynamic changes may temporarily create an arrhythmogenic window of calcium overload, during which the delayed afterdepolarizations and ectopic beats occur. To further elucidate this 
relation, we studied the effect of different interventions that modulate the inotropic state; 1) ouabain, 2) fixed rate pacing, and 3) ryanodine.

1) Partial inhibition of the $\mathrm{Na}^{+} / \mathrm{K}^{+}$pump by ouabain results in a positive inotropic effect. ${ }^{50}$ This is attributed to an increase intracellular calcium loading via an increased intracellular sodium due to (partial) inhibition of the $\mathrm{Na}^{+} / \mathrm{K}^{+}$pump. ${ }^{50}$ When ouabain administration results in intracellular calcium overload, the sarcoplasmic reticulum oscillatory releases calcium in the cytosol, thereby eliciting a transient inward current that is responsible for the delayed afterdepolarization. ${ }^{47,50}$ The therapeutic dosage of ouabain used in this study, resulted in a positive inotropic effect (increase in pre- and post-pacing $+\mathrm{LV} \mathrm{dP} / \mathrm{dt}$ ) both at acute and chronic atrioventricular-block.

However, at 6 weeks of complete atrioventricular-block, the arrhythmogenic window increased after administration of this therapeutic dosage of ouabain, i.e. more pacing trains resulted in the induction of delayed afterdepolarizations and ectopic beats. This supports the hypothesis that the hypertrophied heart is more susceptible to interventions aimed at modulating intracellular calcium.

2) During fixed rate pacing at chronic atrioventricular-block, we found a decrease in $+\mathrm{LV}$ $\mathrm{dP} / \mathrm{dt}$ at similar left ventricular end diastolic pressure and a decrease in post-pacing-potentiation. This corresponded with suppression of all ectopic beats and delayed afterdepolarizations, thereby suggesting that intracellular calcium decreases during fixed rate pacing. In normal myocardium, increasing the heart rate is associated with an increase in contractile force. ${ }^{14,16,21}$ In contrast, the decrease in contractile force during fixed rate pacing at a relative rapid heart rate which we found in the present study, shows similarities to the reversed force frequency relation in failing myocardium. ${ }^{14,16,21}$ Using aequorin measurements, Pieske et al demonstrated that the positive force frequency relation results from frequency dependent increases in intracellular calcium transients, while the negative force frequency relation in failing hearts are associated with a decrease in free intracellular calcium concentration. ${ }^{21}$ However, it has to be noted that in heart faifure, the overall contractile function is severely depressed, while we find at chronic atrioventricular-block higher pre- and post-pacing $+\mathrm{LV} \mathrm{dP} / \mathrm{dt}$ both during idioventricular thythm and during fixed rated pacing. An extensive investigation to the force frequency relation in these dogs is currently undertaken.

3) Ryanodine has been suggested to specifically block the sarcoplasmic reticulum calcium release channel, ${ }^{51}$ causing a negative inotropic action. ${ }^{52,53}$ Additionally, blockade of the SR calcium release channel will abolish delayed afterdepolarizations and thus eliminate related ectopic beats. ${ }^{51}$ In selected dogs with triggered arrhythmias, the 
pronounced negative inotropic effect of ryanodine depressed post-pacing-potentiation and abolished all ectopic activity and delayed afterdepolarizations.

\section{Clinical implications}

Extrapolation from data derived from experimental animals to the human setting should always be done with great caution. It is known that patients with left ventricular hypertrophy have an increased incidence of arrhythmias. ${ }^{1-3}$ We can hypothesize that these patients are more prone to pro-arrhythmic effects when patients are treated with positive inotropic agents. This seems to be supported by the results of the Digitalis Investigation Group, ${ }^{54}$ who found a trend to a decreased mortality attributed to worsening heart failure, but no change overall mortality, possibly related to an increased mortality due to sudden (arrhythmic) death. ${ }^{54}$

Our results also support the intriguing possibility that in the hypertrophied heart ventricular arrhythmias based on other mechanisms like reentry may induce delayed afterdepolarization-dependent triggered arrhythmias.

\section{Limitations}

In this study, we only measured at 2 time points and we do not know to what extent the observed phenomena relate to structural and neurohumoral adaptations in time. Previous studies have indicated that there is no relation between the amount of hypertrophy and the duration of complete atrioventricular-block after the first 2 weeks of atrioventricular-block. ${ }^{11,13}$ We have found similar results. With regard to neurohumoral changes, we have found a transient elevation of plasma ANF, norepinephrine and angiotensin II. However, at 6 weeks of atrioventricular-block, all these parameters had returned to control values (chapter 5). ${ }^{25}$

Although we speculated about alterations in intracellular calcium handling, and modulating intracellular calcium with fixed rate pacing, ouabain and ryanodine, we did not measure calcium transients to prove changes in intracellular calcium.

In addition to alterations in intracellular calcium handling at the level of the cardiomyocyte, other factors can play a role in the alterations in contractile function including the sensitivity of the cardiac contractile proteins and the interstitial tissue of the myocardium.

\section{General conclusions}

The dog with chronic complete atrioventricular-block seems able to maintain ventricular contractile function during idioventricular rhythm. However, the increased inotropic state predisposes the hypertrophied heart to an increased susceptibility to delayed 
afterdepolarization related triggered arrhythmias during positive inotropic interventions.

\section{Acknowledgments}

This study was supported in part by a grant from the Wynand N. Pon Foundation, Leusden, The Netherlands and by a grant from the Bekales Foundation, Vaduz, Liechtenstein. The authors would like to thank the Bakken Research Center, Maastricht, The Netherlands for supplying the electrodes.

\section{References}

1. Levy D. Echocardiogaphically detected left ventricular hypertrophy: prevalence and risk factors. Ann Int Med 1988; 108: 7-13.

2. Kannel WB, Gordon T, Offutt D. Left ventricular hypertrophy by electrocardiogram. Prevalence, incidence and mortality in the Framingham Study. Amm Int Med 1969; 71: 89-105.

3. Swynghedauw B, Chevalier B, Charlemagne D, Mansier P, Carré F. Cardiac hypertrophy, arthythmogenicity and the new myocardial phenotype. II. The cellular adaptational process. Cardiovasc Res 1997; 35: 6-12.

4. Gaasch WH, Zile MR, Hoshino PK, Apstein CS, Blaustein AS. Stress-shortening relations and myociardial blood flow in compensated and failing canine hearts with pressure overload hypertrophy. Circulation 1989; 79: 872-883.

5. Hittinger L, Patrick $T$, thara $T$, Hasebe $N$, Shen $Y T$, Kalthof $B$, Shannon RP. Exercise induces cardiac dysfunction in both moderate, compensated and severe hypertrophy. Circulation 1994; 89 : 2219-2231.

6. Carrabello BA, Zile MR, Tanaka R, Cooper IV G. Left ventricular hypertrophy due to volume overload versus pressure overload. Am J Physiol 1992; 263: H1137-H1144.

7. Spann JF, Buccino RA, Sonnenblick EH, Braunwald E. Contractile state of the cardiac muscle obtained from cats with experimentally produced right ventricular hypertrophy. Circ Res 1967;21: 341-354.

8. Wusten B, Flameng W, Wirkler B, Schaper W. Role of cardiac contractility in hypertrophy from chronic volume loading. Cardiovasc Res 1977; 11: 132-140.

9. Turina $\mathrm{M}_{3}$ Bussman WD, Krayenbuhl HP. Contractility of the hypertrophied canine heart in chronic vollume overload. Cardiovasc Res 1969; 3: 486-495.

10. Urabe $Y$, Mann DL, Kent RL, Nakano K, Tomanek RJ, Carabello B, Cooper IV G. Cellular and ventricular contractile dysfunction in experimental canine mitral regurgitation. Circulation Res 1992 ; 70: 131-147.

11. Starzl TE, Gaertner RA, Baker RR. Acute complete heart block in dogs. Circulation 1955; 12: 8289.

12. Newman WH. Contractile state of hypertrophied left ventricle in long-standing volume overload. Am J Physiol 1978; 234: H88-H93.

13. Brockman SK. Cardiodynamics of complete heart block. Am I Cardiol 1965; 16:72-83.

14. Hasenfuss $G$, Holubarsch $C$, Hermann HP, Astheimer K, Pieske B, Just H. Influence of the force frequency relationship on haemodynamics and left ventricular function in patients with non-failing hearts and in patients with dilated cardiomyopathy. Eur Heart J 1994; 15: 164-170. 
15. Fujii AM, Gelpi $\mathbb{R J}$, Mirsky I, Vatner SF. Systolic and diastolic dysfunction during atrial pacing in conscious dogs with left ventricular hypertrophy. Circ Res 1988; 62: 462-470.

16. Feldman MD, Alderman JD, Aroesty JM, Royal HD, Ferguson JJ, Owen RM, Grossman W, McKay RG. Depression of systolic and diastolic myocardial reserve during atrial pacing tachycardia in patients with dilated cardiomyopathy. J Clin Invest 1988; 82: 1661-1669.

17. Liu CP, Ting CT, Lawrence W, Maughan L, Chang M-S, Kass DA. Diminished contractile response to increased heart rate in intact human left ventricular hypertrophy. Circulation 1993;88: $1893-1906$.

18. Vatner DE, Sato $N$, Kiuchi K, Shannon RP, Vatner SF. Decrease in myocardial ryanodine receptors and altered excitation-contraction coupling early in the development of heart failure. Circulation $1994 ; 90: 1423-1430$.

19. Seed WA, Noble MIM, Walker JM, Miller GAH, Pidgeon J, Redwood D, Wanless R, Franz MR, Schoettler M, Schaeffer J. Relationships between beat-to-beat interval and the strength of contraction in the healthy and the diseased human heart. Circulation 1984; 70:799-805.

20. Morgan JP, Erny RE, Allen PD, Grossman W, Gwathmey JK. Abnormal intracellular calcium handling as a major cause of systolic and diastolic dysfunction in ventricular myocardium from patients with heart failure. Circulation 1.990; 81: III21-11132.

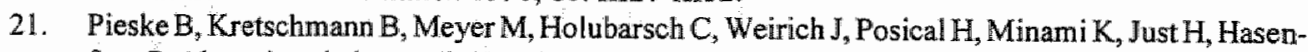
fuss $\mathrm{G}$. Alterations in intracellular calcium handling associated with the inverse force-frequency relation in human dilated cardiomyopathy. Circulation 1995; 92: 1169-1178.

22. Aronson RS, Ming Z. Cellular mechanisms of arrhythmias in the hypertrophied and failing myocardium. Circulation 1993; 87: VII76-VII83.

23. Marban E, Robinson SW, Wier GW. Mechanisms of arrhythmogenic delayed and early afterdepolarizations in ferret ventricular muscle. J Chin Invest 1986; 78: 1185-1192.

24. Wusten $B$, Flameng $W$, Schaper W. Cardiac function in the chronically volume-overloaded canine heart. Basic Res Cardiol 1977; 72: 172-177.

25. Vos MA, van der Zande J, Verduyn SC, de Groot SHM, Kulcsár A, Cleutjens A, van Bilsen M, Daemen $M$, Wellens HJJ. Physiologic hypertrophy after creation of complete atrioventricular block (abstract). Eur Heart J 1996; 17: 549.

26. Verduyn SC, Vos MA, Van der Zande J, De Groot SHM, Allessie MA, Wellens HJJ. Biventricular hypertrophy facilitates the occurence of acquired torsade de pointes arrhythmias in dogs (abstract). Circulation 1995; 92: I504-I505.

27. Scherlag BJ, Kosowsky BD, Damato AN. Technique for ventricular pacing from the His bundle of the intact heart. J Appl Physiol 1967; 22: 584-587.

28. Franz MR, Chin MC, Sharkey HR, Griffin JC, Scheinman MM. A new single catheter technique for simultaneous measurement of action potential duration and refractory period in vivo. $J \mathrm{Am} \mathrm{Coll}$ Cardiol 1990; 16: 878-886.

29. De Groot SHM, Vos MA, Gorgels APM, Leunissen JDM, Van der Steld BJ, Wellens HJJ. Combining monophasic action potential recordings with pacing to demonstrate delayed afterdepolarizations and triggered arrhythmias in the intact heart. Circulation 1995; 92: 2697-2704.

30. Aubert AE. In vivo comparison between two tip pressure transducer systems. Int $J$ Clin Monitor Computing 1995; 12:77-83.

31. Cranefield PF, Aronson R: Cardliac arrhythmias: The role of triggered activity and other mechanisms. New York., Nt Kisco, New York, Futura publishing Co,, 1988.

32. Levine JH, Spear JF, Gaurnieri T, Weisfeldt ML, de Langen CDJ, Becker LC, Moore EN. Cesium chloride-induced long QT syndrome: demonstration of afterdepolarizations and triggered activity
in vivo. Circulation 1985; 72:1092-1103. 
33. Schouten VJA, Vliegen HW, van der Laarse A, Huysmans HA. Altered calcium handling at normal contractility in hypertrophied rat heart. $J$ Mol Cell Cardiol $1990 ; 22: 987-998$.

34. Ben-David I, Zipes DP, Ayers GM, Pride HP. Canine left ventricular hypertrophy predisposes to ventricular tachycardia induction by phase 2 early afterdepolarizations after administration of BAY K 8644.J Am Coll Cardiol 1992; 20: 1576-1584.

35. Laks MM, Morady F, Garner D, Swan HJC. Relation of ventricular volume, compliance, and mass in normal and pulmonary arterial banded canine heart. Cardiovasc Res 1972; 6: 187-198.

36. Sabbah HN, Stein PD, Kono T, Gheorghiade M, Levine TB, Jafri S, Hawkins ET, Goldstein S. A canine model of chronic heart failure produced by multiple sequential coronary microemboliza tions. Am J Physial 1991; 260: H1379-H1384.

37. Perreault CL, Shannon RP, Komamura K, Vatner SF, Morgan JP. Abnormalities in intracellular calcium regulation and contractile function in myocardium from dogs with pacing-induced heart faillure. J Clin Invest 1992; 89: 932-938.

38. Cooper MW. Postextrasystolic potentiation: do we really know what it means and how to use it? Circulation 1993; 88: 2962-2971.

39. ter Keurs HEDJ, Schouten VJA, Bucx JJ, Mulder BM, de Tombe PP. Excitation-contraction coupling in myocardium: implications of calcium release and $\mathrm{Na}+\mathrm{Ca} 2+$ exchange. $\mathrm{Can} J \mathrm{Physiol}$ 1987; 65: 619-626.

40. Meijler FJ, van den Boogaard F, van der Tweel LH, Durrer D. Postextrasystolic potentiation in the isolated rat heart. Am J Physiol 1962; 202: 631-635.

41. Rosen MR. Is the response to programmed electrical stimulation diagnostic of mechanisms for arrhythmias? Circulation 1986; 73: II18-1127.

42. Vos MA, Gorgels AP, de Wit B, Drenth JP, van Deursen RT, Leunissen JD, Wellens HJ. Premature escape beats. A model for triggered activity in the intact heart? Circulation 1990; 82:213-224.

43. Aronson RS. Afterpotentials and triggered activity in hypertrophied myocardium from rats with renal hypertension. Circ Res 1981; 48: 720-727.

44. Charpentier F, Bandet $S$, Le Marec $H$. Triggered activity as a possible mechanism for arrhythmias in ventricular hypertrophy. $P A C E 1991 ; 14: 1735-1741$

45. Samson RA, LeeH-C. Delayed afterdepolarizations and triggered arrhythmias in hypertrophic cardiomyopathic hearts. J Lab Clin Med 1994; 124: 242-248.

46. Vermeulen JT, McGuire MA, Opthof T, Coronel R, de Bakker JM, Klopping C, Janse MJ. Triggered activity and automaticity in ventricular trabeculae of failing human and rabbit hearts. Cardiovasc Res 1994; 28 : 1547-1554.

47. Rosen MR. Cardiac arrhythmias and antiarrhythmic drugs: recent advances in our understanding of mechanism. J Cardiovasc Electrophysiol 1995; 6: 868-879.

48. Fedida $D$, Noble $D$, Rankin $A C$, Spindler AJ. The arrhythmogenic transient inward current lti and related contraction in isolated guinem-pig ventricular myocytes. J Physiol (Lond) 1987; 392: 523 542.

49. Berlin JR, Cannell MB, Lederer WJ. Cellular origins of the transient inward current in cardiac myocytes. Role of fluctuations and waves of elevated intracellular calcium. Circ Res $1989 ; 65: 1$ IS126.

50. Smith TW. Digitalis. Mechanisms of action and clinical use. $N$ Engl J Med 1988; $318: 358-365$.

51. Marban E, Wier WG. Ryanodine as a tool to determine the contributions of calcium entry and calcium release to the calcium transient and contraction of cardiac Purkinje fibers. Circ Res 1985; 56 : 133-138.

52. Hadju S, Leonard E. Action of ryanodine on mammalian cardiac muscle. Effects on contractility, and reversal of digitalis induced ventricular arrhythmias. Circ Res 1961; 9:1291-1298. 


\section{Chapter 6}

53. de Groot $M$, Vos $M$, Leunissen J, Wellens $H$. The effect of ryanodine determines the rate of delayed afterdepolarization dependent ventricular tachycardia. (abstract). PACE 1996; 16: 604.

54. The Digitalis Investigation Group. The effect of digoxin on mortality and morbidity in patients with heart failure. New Engl J Med 1997; 336:525-533. 


\title{
Chapter 7
}

\section{Hypertrophy in the canine heart leads to an attenuated force-frequency relation with no signs of left or right ventricular pump failure}

\author{
Marieke de Groot \\ Marc Vos \\ Jan Schreuder \\ Mirella Molenschot \\ Jet Leunissen \\ Hein Wellens
}




\section{Abstract}

Introduction: In dogs, chronic complete atrioventricular block leads to biventricular hypertrophy. Many features of this hypertrophy process still remain unresolved, especially it is unclear to what extent the hypertrophied heart is able to maintain its pump function during chronotropic stress.

Methods: In anesthetized dogs, we serially ( 0 and 6 weeks of atrioventricular block) determined the left and right ventricular function during baseline and chronotropic stress (force-frequency relation).For this purpose, we recorded pressure signals in both ventricles and determined cardiac output at paced cycle lengths varying between 1250 and 300 ms.

Results: During idioventricular rhythm, the cardiac output was higher at 6 weeks of complete atrioventricular block than at acute atrioventricular block $(2.4 \pm 0.5$ versus $2.0 \pm 0.3 \mathrm{l} / \mathrm{min}$ respectively). Both left and right ventricular end-diastolic pressure had decreased at 6 weeks of atrioventricular-block, while both the rate of pressure-rise $(+\mathrm{dP} / \mathrm{dt})$ and the end-systolic pressures had increased in both ventricles. At 6 weeks of atrioventricular block, the force frequency relation was inversed, being highest at a paced cycle length of $1250 \mathrm{~ms}$. However, the force frequency relation was not depressed and remained higher at all paced cycle lengths.

Conclusion: The dogs with chronic atrioventricular block show an inversed but not depressed force frequency relation.

\section{Introduction}

In the normal myocardium, an increase in heart rate usually results in an increase in contractile force, i.e. a positive inotropic response. This phenomenon, known as "forcefrequency relation", "positive staircase", or "treppe", was originally described by Bowditch over 120 years ago. ${ }^{1}$ Subsequently this observation was confirmed by other investigators in cardiac muscle strips and isolated ventricular myocytes, as well as in whole hearts from most mammals, including humans. ${ }^{2-10}$

In contrast, in the failing myocardium, frequency potentiation of contractile force is absent, attenuated, or even reversed. ${ }^{2,4,6-10}$ In the failing left ventricle, the contractile force was highest at low stimulation rates and decreased at higher frequencies. $2,4,7-10$ This reversal of the force-frequency relation is related to the stage of heart failure, being most pronounced in severe congestive heart failure. ${ }^{11}$ 
Although the left ventricular force-frequency relation has been extensively studied in normal and failing myocardium, limited studies have focused on the frequency potentiation of hypertrophied hearts with normal baseline left ventricular function. ${ }^{12}$ Additionally, when compared to the left ventricle, the right ventricular force-frequency relation remains poorly characterized, ${ }^{6,13}$ particularly in vivo.

In dogs, chronic complete atrioventricular-block leads to biventricular hypertrophy. ${ }^{14-20}$ Although some authors described the development of heart failure in these dogs, 14,16 others, including us, found at least a maintained left ventricular function at any evaluated state of hypertrophy up to 3 years of complete atrioventricular-block. ${ }^{15,17,18,20,21}$ However, we noticed in previous experiments that the left ventricular rate of pressure rise $(+\mathrm{LV} \mathrm{dP} / \mathrm{dt})$ decreased when the heart was paced at $100-120$ beats per minute, ${ }^{21}$ possibly suggesting an altered left ventricular force-frequency relation. Therefore, it was the purpose of this study, to evaluate the effect of biventricular hypertrophy induced by atrioventricular-block on the force-frequency relation of both ventricles.

\section{Methods}

All experiments were performed in accordance with the "Guiding principles in the care and use of laboratory animals" (NIH publication \#86-23, 1996) as approved by the American Physiological Society and under the Dutch Law on Animal Experimentation and the European Directive for the Protection of Vertebrate Animals used for Experimental Purposes. Additionally, all experiments were approved by the Animal Investigation Committee of the Maastricht University.

\section{General}

The studies were performed in 10 anesthetized adult mongrel dogs (mean body weight of $27 \pm 5 \mathrm{~kg}$ ) of either sex. Eight dogs were studied at the time of acute atrioventricularblock and after 6 weeks of chronic complete atrioventricular-block. Four of these dogs were studied at both times.

After overnight fasting, premedication $(0.2 \mathrm{ml} / \mathrm{kg}$ ) was given intramuscularly using a drug mixture containing $10 \mathrm{mg}$ oxycodan $\mathrm{HCl}, 1 \mathrm{mg}$ acepromazine and $0.5 \mathrm{mg}$ atropine sulfate per $\mathrm{ml}$. Anesthesia was induced by intravenous sodium pentobarbital $(20 \mathrm{mg} / \mathrm{kg}$ i.v., Nembutal ${ }^{\circledR}$, Sanofi) The dogs were artificially ventilated (Puimomat respirator, Dräger), through a cuffed endotracheal tube with a mixture of oxygen, nitrous oxide and halothane (vapor concentration 0.5-1\%). Ventilation was adjusted on the basis of continuous monitoring of the carbon dioxide concentration of the expired air. Proper care 

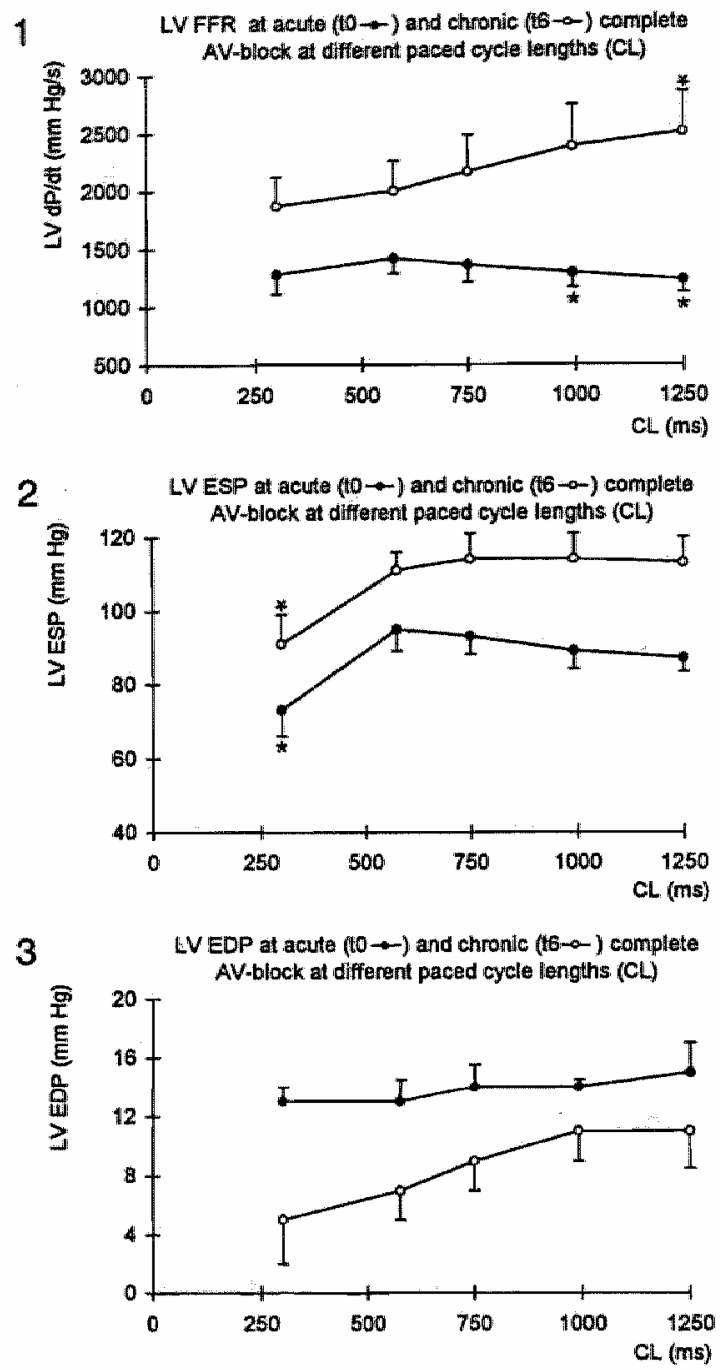

Figure 1: The effect of different paced cycle lengths (CL) on left ventricular rate of pressure rise ( $+\mathrm{LV}$ $\mathrm{dP} / \mathrm{dt}$, panel 1), left ventricular end-systolic pressure (LV ESP, panel 2) and left ventricular end-diastolic pressure (LV EDP, panel 3) at acute and chronic atrioventricular-block (AV-block). Data presented as mean \pm SEM, * indicates $\mathrm{p}<0.05$ in comparison with $\mathrm{CL} 575 \pm 25 \mathrm{~ms}$.

During chronic AV-block the force-frequency-relation (FFR) is significantly higher than at acute AV-black. At both times, the LV ESP behaves concordant to the LV dP/dt. In contrast the LV EDP curve has significantly decreased at chronic AV-block and declines during faster pacing. 
was taken during and after the experiments, including an antibiotic ( $1000 \mathrm{mg}$ ampicillin) pre- (i.v.) and post-operatively (i.m.) and analgesics $(0.015 \mathrm{mg} / \mathrm{kg} \mathrm{i.m}$. buprenorfine,

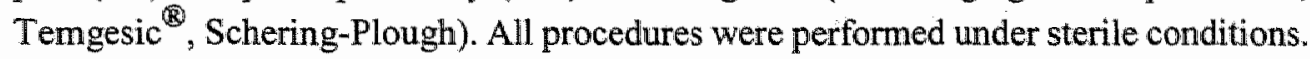
A thermal mattress was used to maintain body temperature at approximately $37^{\circ} \mathrm{C}$.

\section{Atrioventricular-block}

To create atrioventricular-block, a right sided thoracotomy was performed through the fourth or the fifth intercostal space. During this procedure an epicardial screw-in electrode (Bakken Research Center, Medtronic, Maastricht) was placed on the apex of the left ventricle. Thereafter, complete atrioventricular-block was made by injecting $37 \%$ formaldehyde in the atrioventricular-node ${ }^{22}$ and the thorax was closed.

\section{Experimental procedures and hemodynamic measurements}

During the experiments, six surface ECG leads were simultaneously registered and the values of RR intervals were instantaneously displayed on a monitor screen using a computerized QRS-complex detecting system.

To evaluate the effect of different pacing rates on left and right ventricular pressure and peak rate of pressure rise $(\mathrm{dP} / \mathrm{dt} \max )$, the left and right ventricular pressures were measured with a single micromanometer transducer catheter $(7.5$ and $7 \mathrm{~F}$ respectively, Sentron, Roden, The Netherlands). Prior to insertion the pressure sensors were emerged in a $37^{\circ} \mathrm{C} 0.9 \% \mathrm{NaCl}$ solution to minimize temperature drift. The Sentron interface (Type No 811-000/A) was used for calibration of the pressure transducer. The pressure catheter was positioned in the left ventricle via the carotid artery and in the right ventricle via the jugular vein.

Cardiac output determination with the thermodilution method was repeated 3 times and the average value was used. ${ }^{23}$ For this purpose a Swan-Ganz catheter was positioned in the pulmonary artery, just above the valves, via the external jugular vein. All catheters were placed under fluoroscopic control. All signals were sampled at a rate of $1 \mathrm{kHz}$ per signal and stored on the hard disc of the computer. Ventricular pressure curves and cardiac output were obtained during 1) sinus rhythm, 2) acute atrioventricular-block and 3) chronic atrioventricular-block.

In 2 dogs, a conductance catheter ( $7.5 \mathrm{~F}$, Webster Labs) was placed in the apex of the left ventricle at acute and chronic ( 6 weeks) atrioventricular-block, through the carotid artery. The conductance catheter was connected to a Leycom Sigma-5DF signal conditioner processor (CardioDynamics, Zoetermeer, the Netherlands) to measure left ven- 
tricular volume. A correct position of the catheter was assumed if the signals of at least the 4 most distal segments displayed a typical left ventricular volume tracing. ${ }^{24}$ Conductance catheter stroke volume was calibrated by thermodilution stroke volume (SwanGanz through the jugular vein). Pressure-volume relations were determined at 2 paced cycle lengths: $460-600 \mathrm{~ms}$ (equal to the pre-existent sinus rhythm cycle length) and 1200 $\mathrm{ms}$ (slightly faster than the idioventricular rhythm). At the completion of all procedures, all catheters were removed and the vessels were sutured.

\section{Determination of the frequency adaptation}

To study the force-frequency relation, the heart was paced at different cycle lengths from the left ventricular epicardial electrode with a custom build programmable stimulator (Maastricht University), that delivers unipolar stimuli synchronous to the QRS complexes with a pulse width of $2 \mathrm{~ms}$ and a stimulus strength of twice the diastolic threshold. As indifferent electrode a needle was placed through the skin.

The pacing protocol used cycle lengths of $300,575 \pm 25,750,1000$ and $1250 \mathrm{~ms}$. At all cycle lengths, pacing was performed for at least 3 minutes to obtain a steady state. The right and left ventricular pressure and the cardiac output were determined at each cycle length. Stroke volume was determined by dividing the cardiac output by the heart rate.

\section{Data analysis}

For off-line analysis of the recorded signals a special software program (ECGVIEW) was used. The positive $\mathrm{dP} / \mathrm{dt} \max (\mathrm{mm} \mathrm{Hg} / \mathrm{s})$, end-diastolic pressure and end-systolic pressure were measured for both ventricles during sinus rhythm, idioventricular rhythm and the different paced rhythms. The values were calculated as the average of 5 consecutive beats.

\section{Statistics}

All data are presented as mean \pm standard error of the mean (SEM). Repeated measures ANOVA followed by Bonferroni's t-test (for more than two-group analysis) and twotailed t-test (for two-group comparison) were used to determine statistical significance. A p-value $<0.05$ was accepted as statistically significant. 


\section{Results}

\section{Sinus rhythm}

The cycle length during sinus rhythm was $571 \pm 17 \mathrm{~ms}$ with a cardiac output of $2.7 \pm 0.2$ $\mathrm{V} / \mathrm{min}$, resulting in a stroke volume of $26 \pm 5 \mathrm{ml}$. At that time, the $+\mathrm{LV} \mathrm{dP} / \mathrm{dt}$ was $1306 \pm 88$ $\mathrm{mm} \mathrm{Hg} / \mathrm{s}$, the left ventricular end-diastolic pressure $11 \pm 1 \mathrm{~mm} \mathrm{Hg}$ and the left ventricular end-systolic pressure was $98 \pm 4 \mathrm{~mm} \mathrm{Hg}$. For the right ventricle the $\mathrm{dP} / \mathrm{dt}$ was $303 \pm 20$ $\mathrm{mm} \mathrm{Hg} / \mathrm{s}$, the end-diastolic pressure $3 \pm 1 \mathrm{~mm} \mathrm{Hg}$ and the end-systolic pressure $18 \pm 1 \mathrm{~mm}$ $\mathrm{Hg}$.

\section{Acute atrioventricular-block}

\section{Baseline}

After acute complete atrioventricular-block the cycle length of the idioventricular rhythm was $1686 \pm 84 \mathrm{~ms}(p<0.05)$. Although stroke volume increased to $44 \pm 4 \mathrm{ml}$ after the creation of atrioventricular-block, the cardiac output declined to $2 \pm 0.3$ liter $/ \mathrm{min}(\mathrm{p}<0.05$, for both). This was accompanied by an increased end-diastolic pressure of both ventricles (left ventricle: $15 \pm 2$, right ventricle: $7 \pm 1 \mathrm{~mm} \mathrm{Hg}, \mathrm{p}<0.05$, for both), while all other parameters remained similar (table 1).

\section{Left ventricular force-frequency relation}

Decreasing the paced cycle length from 1250 to $575 \pm 25 \mathrm{~ms}$, resulted in an increase in the $+\mathrm{LV} \mathrm{dP} / \mathrm{dt}$ (from $1232 \pm 112$ to $1420 \pm 134 \mathrm{~mm} \mathrm{Hg} / \mathrm{s}$; table 1, figure 1; panel 1), while the left ventricular end-diastolic did not change (figure 1; panel 3).

Further decreasing the paced cycle length to $300 \mathrm{~ms}$ resulted in a depression of $+\mathrm{LV}$ $\mathrm{dP} / \mathrm{dt}$ (to $1281 \pm 168 \mathrm{~mm} \mathrm{Hg} / \mathrm{s}$ ) and the left ventricular end-systolic pressure (to $73 \pm 7 \mathrm{~mm}$ $\mathrm{Hg}$, table 1 and figure 1 ; panel 1 and 2). Consequently, the $\mathrm{dP} / \mathrm{dt}$ showed a biphasic adaptation response to the increase in heart rate (figure 1; panel 1).

\section{Right ventricular force-frequency relation}

As in the left ventricle, decreasing the paced cycle length from 1250 to $575 \pm 25 \mathrm{~ms}$ resulted in an increase in the right ventricular rate of pressure rise ( $+\mathrm{RV} \mathrm{dP} / \mathrm{dt}$; from $218 \pm 11$ to $314 \pm 34 \mathrm{~mm} \mathrm{Hg} / \mathrm{s}$, table 1, $\mathrm{p}<0.05$, figure 2; panel 1). The end-diastolic pressure decreased from $6 \pm 2$ at paced cycle length $1250 \mathrm{~ms}$ to $4 \pm 2 \mathrm{~mm} \mathrm{Hg}$ at paced cycle length $575 \pm 25 \mathrm{~ms}$, while the end-systolic pressure remained unaltered (table 1, figure 2; panel 2 and 3 ). 
Table 1: Overview of the effect of different heart rates on left and right ventricular hemodynamics during sinus rhythm and all acute and chronic atrioventricular-block

\begin{tabular}{|c|c|c|c|c|c|c|c|}
\hline \multicolumn{4}{|c|}{ Sinus Rhythm } & \multicolumn{3}{|c|}{$\leftarrow$ Acute AV-block $\rightarrow$} & \multirow[b]{2}{*}{ CL 1250} \\
\hline & & IVR & CL 300 & CL 575 & CL 750 & CL 1000 & \\
\hline LVEDP & $11 \pm 1$ & $15 \pm 2$ & $13 \pm 1$ & $13 \pm 2$ & $14 \pm 2$ & $14 \pm 1$ & $15 \pm 2$ \\
\hline LV ESP & $98 \pm 4$ & $89 \pm 4$ & $73 \pm 7$ & $95 \pm 6$ & $93 \pm 5$ & $89 \pm 5$ & $87 \pm 4^{*}$ \\
\hline $\mathrm{LV} d \mathrm{P} / \mathrm{dt}$ & $1306 \pm 88$ & $1327 \pm 143$ & $1281 \pm 168$ & $1420 \pm 134$ & $1366 \pm 151$ & $1291 \pm 128$ & $1232 \pm 112^{*}$ \\
\hline RV EDP & $3 \pm 1$ & $7 \pm 1$ & $3 \pm 1$ & $4 \pm 2$ & $4 \pm 2$ & $5 \pm 2$ & $6 \pm 2^{*}$ \\
\hline RV ESP & $18 \pm 1$ & $22 \pm 2$ & $20 \pm 1$ & $0 \pm 2$ & $21 \pm 2$ & $21 \pm 2$ & $22 \pm 2$ \\
\hline $\mathrm{RV} \mathrm{dP} / \mathrm{dt}$ & $303 \pm 20$ & $302 \pm 51$ & $378 \pm 29^{*}$ & $314 \pm 34$ & $273 \pm 30$ & $245 \pm 17^{*}$ & $218 \pm 11$ \\
\hline $\mathrm{CO}$ & $2.7 \pm 0.2$ & $2 \pm 0.3$ & $2 \pm 0.3^{\circ}$ & $3 \pm 0.2$ & $2 \pm 0.2$ & $2 \pm 0.2^{*}$ & $2 \pm 0.2^{*}$ \\
\hline \multirow[t]{3}{*}{ SV } & $26 \pm 5$ & $44 \pm 4$ & $10 \pm 11^{*}$ & $26 \pm 2$ & $30 \pm 2$ & $37 \pm 2^{*}$ & $38 \pm 3^{*}$ \\
\hline & & \multicolumn{6}{|c|}{$\leftarrow$ Chronic AV-block $\rightarrow$} \\
\hline & & IVR & CL 300 & CL 575 & CL 750 & CL 1000 & CL 1250 \\
\hline LV EDP & & $11 \pm 2.5$ & $5 \pm 3^{\text {F }}$ & $7 \pm 2$ & $9 \pm 2$ & $11 \pm 2$ & $11 \pm 2.5$ \\
\hline LV $\mathbb{E S P}$ & & $12 \pm 7^{4 t}$ & $91 \pm 8$ & $111 \pm 5$ & $114 \pm 7$ & $114 \pm 7$ & $113 \pm 7^{*}$ \\
\hline $\mathrm{LV} \mathrm{dP} / \mathrm{dt}$ & & $2700 \pm 319^{\prime \prime}$ & $1876 \pm 252$ & $2008+255^{4}$ & $2171 \pm 317^{\#}$ & $2385 \pm 356^{\#}$ & $2510 \pm 346^{*}$ \\
\hline RV EDP & & $4 \pm 1$ & $-2 \pm 1^{1 / 4}$ & $1 \pm 1^{\#}$ & $0.3 \pm 1$ & $2 \pm 1$ & $3 \pm 1$ \\
\hline RV ESP & & $31 \pm 2^{* * *}$ & $21 \pm 1$ & $23 \pm 1$ & $24 \pm 2$ & $27 \pm 2^{*}$ & $29 \pm 2^{* * *}$ \\
\hline $\mathrm{RV} \mathrm{dP} / \mathrm{dt}$ & & $620 \pm 44$ " & $536 \pm 43^{*}$ & $581 \pm 74^{*}$ & $563 \pm 68^{*}$ & $563 \pm 56^{\#}$ & $558 \pm 53^{\prime}$ \\
\hline $\mathrm{CO}$ & & $2.4 \pm 0.5$ & $3 \pm 1$ & $3.8 \pm 0.6$ & $3.5 \pm 0.5^{\mathrm{Z}}$ & $3 \pm 0.4^{*}$ & $2.3 \pm 0.2$ \\
\hline SV & & $55 \pm 7.4^{*}$ & $15 \pm 3^{*}$ & $33 \pm 4.4$ & $44 \pm 6.2^{*}$ & $49.6 \pm 7.5^{*}$ & $53 \pm 6.7^{*}$ \\
\hline
\end{tabular}

LV: left ventricular, RV: right ventricular, EDP: end-diastolic pressure (mm Hg), ESP: end-systolic pressure $(\mathrm{mm} \mathrm{Hg}), \mathrm{dP} / \mathrm{dt}$ : rate of pressure rise $(\mathrm{mm} \mathrm{Hg} / \mathrm{s}), \mathrm{CO}$ : cardiac output ( $(\mathrm{min}), \mathrm{SV}$ : stroke volume $(\mathrm{ml})$. IVR: idioventricular rhythm (ms), CL: cycle length (ms). Data presented as mean $\pm S E M, *$ : $p<0.05$ when compared to CL $575 \pm 25 \mathrm{~ms}$.

In contrast to the left ventricle, further decreasing the paced cycle length to $300 \mathrm{~ms}$ resulted in a further increase in $\mathrm{dP} / \mathrm{dt}$, accompanied by a further decline in end-diastolic pressure. Therefore, no biphasic adaptation curve was seen (figure 2; panel 1 and 3 ).

\section{Rate adaptation of stroke volume, cardiac output and pressure-volume relations}

When the heart was paced at a cycle length of $575 \pm 25 \mathrm{~ms}$, cardiac output increased and stroke volume decreased, both normalizing to the sinus rhythm value (table 1 and figure 3). Further acceleration of the heart rate by decreasing the paced cycle length to $300 \mathrm{~ms}$ resulted in a decline of both cardiac output and stroke volume (table 1 and figure 3 ). The pressure-volume relations illustrated that an acute decrease in heart rate from 100-130 to 50 beats per minute, resulted in an increased left ventricular end-diastolic and 

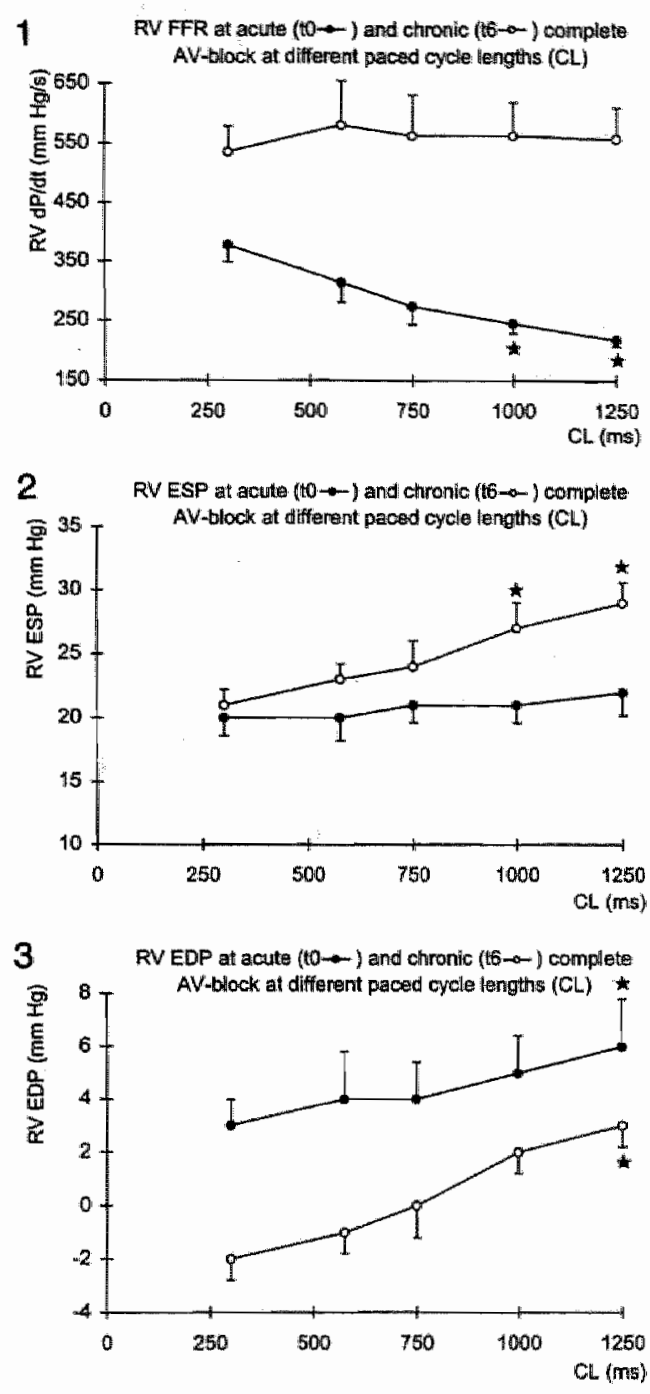

Figure 2: The effect of different paced cycle lengths (CL) on right ventricular rate of pressure rise $(+\mathrm{RV}$ $\mathrm{dP} / \mathrm{dt}$, panel 1), right ventricular end-systolic pressure (RV ESP, panel 2) and right ventricular end-diastolic pressure (RV EDP, panel 3) at acute and chronic atrioventricular-block (AV-block).

Data presented as mean $\pm S E M, *$ : indicates $p<0.05$ in comparison with $\mathrm{CL} 575 \pm 25 \mathrm{~ms}$.

In comparison to acute AV-block, the force-frequency-relation (FFR) is significantly higher at chronic AV-block. At both times, the RV ESP behaves in correspondence with the RV dP/dt. At chronic $A V$-block, the RV EDP curve is significantly decreased and declines during faster pacing. 
end-systolic volume (figure 4). Also in these 2 dogs, the left ventricular end-diastolic pressure was high ( 24 and $16 \mathrm{~mm} \mathrm{Hg}$, figure 4 ) at the paced cycle length of $1200 \mathrm{~ms}$.

\section{Chronic atrioventricular-block}

\section{Baseline}

The cycle length of the idioventricular rhythm at 6 weeks of complete atrioventricularblock was similar to the cycle length at acute complete atrioventricular-block $(1581 \pm 122$ versus $1686 \pm 84 \mathrm{~ms}$, respectively). Now, the end-diastolic pressure of both ventricles had decreased to values similar to those measured during sinus rhythm (table 1). Both the $+\mathrm{LV}$ and the $+\mathrm{RV} \mathrm{dP} / \mathrm{dt}$ had increased, as had the left and right ventricular end-systolic pressure (table 1, $<<0.05$ for all). Finally, at idioventricular thythm, the cardiac output had increased to sinus rhythm values (table 1).

\section{Left ventricular force-frequency relation}

In contrast to acute atrioventricular-block, decreasing the paced cycle length from 1250 to $575 \pm 25 \mathrm{~ms}$ at chronic atrioventricular-block resulted in a depression of the $\mathrm{dP} / \mathrm{dt}$ (table $1, \mathrm{p}<0.05$, figure 1 ; panel 1). This was accompanied by a significant decrease in left ventricular end-systolic pressure, while the left ventricular end-diastolic pressure remained similar (table 1, figure 1; panel 2 and 3).

Again, further decreasing the paced cycle length to $300 \mathrm{~ms}$ resulted in a further decline of the $\mathrm{dP} / \mathrm{dt}$, which was accompanied by a pronounced decrease in end-systolic pressure (table 1 and figure 1; panel 1 and 2). Consequently, the $+\mathrm{LV} \mathrm{dP/dt}$ no longer showed a biphasic adaptation response; instead it was inversed, i.e. it was highest at the longest paced cycle length. Remarkably, the $+\mathrm{LV} \mathrm{dP} / \mathrm{dt}$ and left ventricular end-systolic pressure were significantly higher at chronic atrioventricular-block in comparison to their values at acute atrioventricular-block, while the left ventricular end-diastolic pressure had significantly decreased (table 1 ).

\section{Right ventricular force-frequency relation}

Decreasing the paced cycle length from 1250 to $300 \mathrm{~ms}$ no longer resulted in an increase in $\mathrm{dP} / \mathrm{dt}$ (figure 2; panel 1). At this frequency range, the end-systolic and end-diastolic pressure decreased significantly (from $29 \pm 2$ to $21 \pm 1 \mathrm{~mm} \mathrm{Hg}$ and from $3 \pm 1$ to $-2 \pm 1 \mathrm{~mm}$ $\mathrm{Hg}$, respectively; $\mathrm{p}<0.05$ for both, table 1 , figure 2; panel 2 and 3 ). As for the left ventricle, also in the right ventricle the $\mathrm{dP} / \mathrm{dt}$ was significantly higher at chronic atrioventricularblock in comparison to acute atrioventricular-block. 

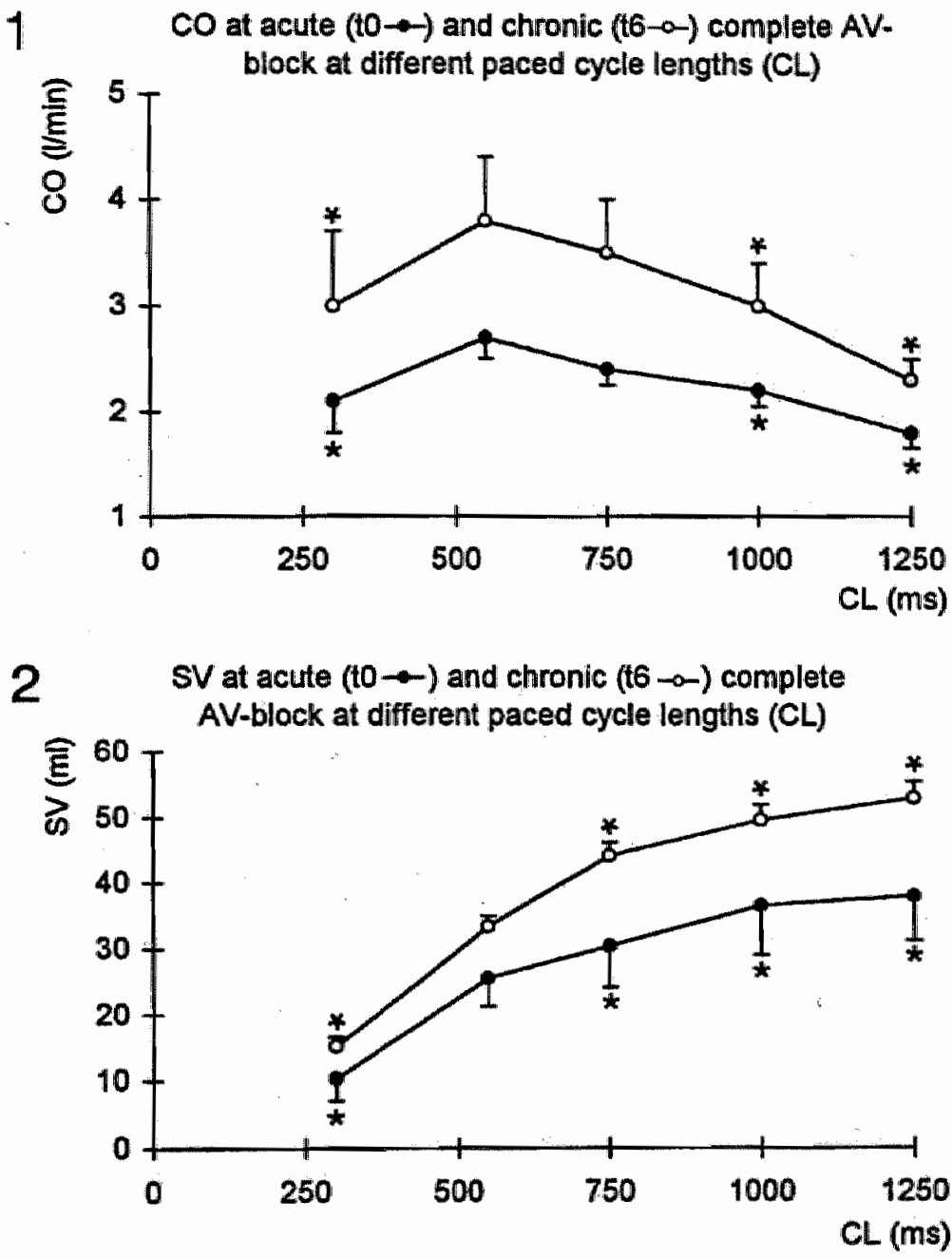

Figure 3: The effect of different paced cycle lengths (CL) on cardiac output ( $\mathrm{CO}$, panel 1) and stroke volume (SV, panel 2) at acute and chronic atriowentricular block (AV-block). Data are presented as mean $\pm S E M, *: p<0.05$, compared to paced CL. $575 \pm 25 \mathrm{~ms}$.

At acute AV-block decreasing the paced cycle length from 1250 to $575 \pm 25 \mathrm{~ms}$ is accompanied by an increase in $C O$. Further decreasing the paced $\mathrm{CL}$ to $300 \mathrm{~ms}$ is associated with a decline in $\mathrm{CO}$. At chronic complete AV-block, the $C O$ behaves similarly but is higher at all times ( $p<0.05$ ).

Both at acute and chronic complete AV-block a decrease in paced cycle length from 1250 to $300 \mathrm{~ms}$ is accompanied by a decrease in SV. However, at chronic AV-block SV is higher at all times $(p<0.05)$. 
ACUTE COMPLETE AV-BLOCK

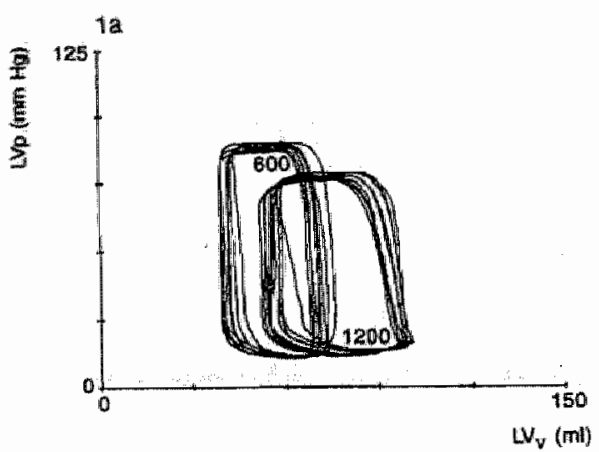

CHRONIC COMPLETE AV-BLOCK

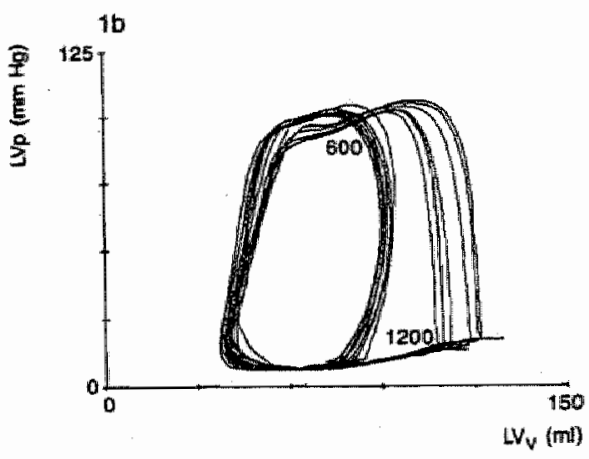

\begin{tabular}{lcc|cc}
\hline & \multicolumn{2}{c|}{ Acute AV-block } & \multicolumn{2}{c}{ Chronic AV-block } \\
\cline { 2 - 5 } Paced CL (ms) & 600 & 1200 & 600 & 1200 \\
\hline ESP (mm Hg) & 88 & 75 & 96 & 89 \\
EDP (mm Hg) & 11 & 16 & 8 & 15 \\
SV (ml) & 32 & 41 & 45 & 62 \\
CO ( (min) & 3.2 & 2.1 & 4.5 & 3.1 \\
EDV (ml) & 70 & 99 & 90 & 114 \\
ESV (mi) & 39 & 58 & 45 & 52 \\
pos LV dP/dt (mm Hg/s) & 1022 & 669 & 1435 & 1604 \\
neg LV dP/dt (mm Hg/s) & -717 & -498 & -1335 & -1178 \\
tau (ms) & 58 & 71 & 30 & 34 \\
PFR (m/s) & 305 & 295 & 391 & 561 \\
PER (m/s) & 188 & 192 & 366 & 372 \\
\hline
\end{tabular}

Figure 4: Pressure-volume relations illustrating the effect of changes in paced cycle length (CL) from 460-600 ms (equal to sinus thythm frequency) to $1200 \mathrm{~ms}$, at acute (panels 1a and 2a) and chronic atrioventricular-block ( $\mathrm{AV}$-block, panels $1 \mathrm{~b}$ and $2 \mathrm{~b}$ ). In the tables under the pressure-volume-loops, the accompanying data are given.

ESP: end systolic pressure, EDP: end diastolic pressure, SV: stroke volume, CO: cardiac output, EDV: end diastolic volume, ESV: end systolic volume, PFR: peak filling rate, PER: peak ejection rate, 460, 600 and $1200 \mathrm{~ms}$ indicate the paced cycle length. 

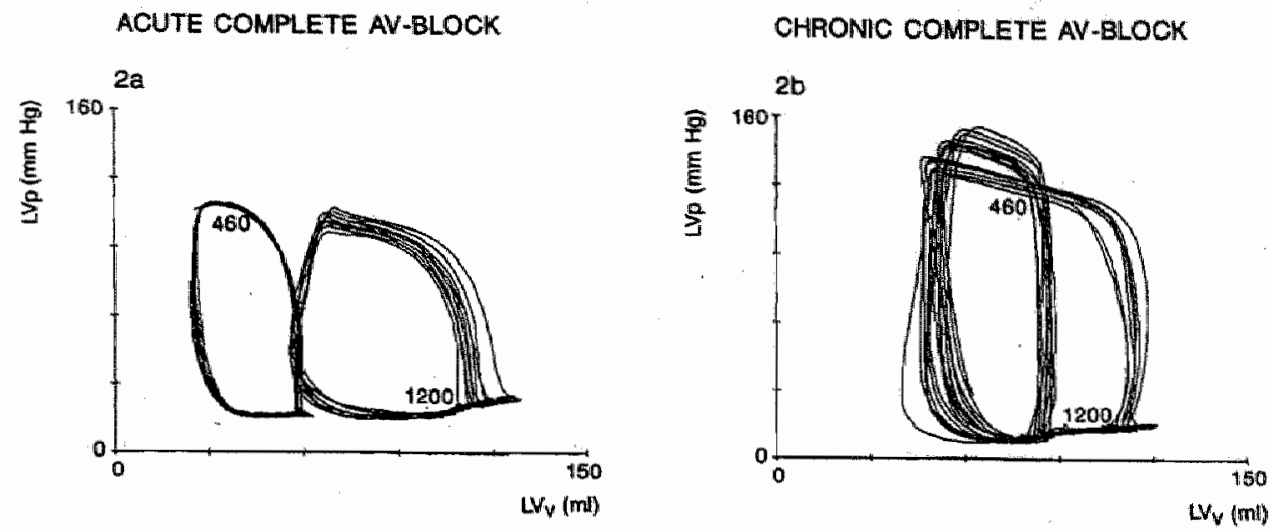

\begin{tabular}{lcc|cc}
\hline & \multicolumn{2}{c|}{ Acute AV-black } & \multicolumn{2}{c}{ Chronic AV-black } \\
\cline { 2 - 5 } Paced CL (ms) & 460 & 1200 & 460 & 1200 \\
\hline ESP (mm Hg) & 113 & 105 & 149 & 137 \\
EDP (mm Hg) & 18 & 24 & 12 & 15 \\
SV (mi) & 33 & 52 & 30 & 62 \\
CO ( $(\mathrm{min})$ & 4.3 & 2.6 & 3.9 & 3.1 \\
EDV (ml) & 81 & 118 & 87 & 112 \\
ESV (ml) & 28 & 66 & 57 & 50 \\
pos LV dP/dt (mm Hg/s) & 1433 & 1270 & 2062 & 2157 \\
neg LV dP/dt (mm Hg/s) & -1380 & -1204 & -2167 & -1912 \\
tau (ms) & 35 & 39 & 31 & 31 \\
PFR (mils) & 427 & 670 & 531 & 656 \\
PER (m/s) & 392 & 515 & 485 & 720 \\
\hline
\end{tabular}

Figure 4 continued: At acute AV-block, decreasing the heart rate is accompanied by: 1 ) an increase in $S V$, 2) a decrease in $C O, 3$ ) an increase in ESV, EDV and EDP and 4) a decrease in ESP. At chronic AV-block, the $\mathrm{CO}, \mathrm{SV}, \mathrm{ESP}$ and $+\mathrm{dP} / \mathrm{dt}$ have increased. Increasing the heart rate from 50 beats per minute to 100-130 beats per minute is accompanied by a marked decrease in EDV while ESV remains similar. 


\section{Rate adaptation of stroke volume, cardiac output and pressure-volume relations}

Comparable to acute atrioventricular-block, also at chronic atrioventricular-block, decreasing the paced cycle length from 1250 to $575 \pm 25 \mathrm{~ms}$ resulted in an increase in cardiac output. At all frequencies, both cardiac output and stroke volume were highest at chronic atrioventricular-block (table $\mathbb{1}$ and figure 3 ).

Also the pressure-volume relations illustrated that systolic ventricular function at the slow heart rate had improved; peak ejection rate, $\mathrm{dP} / \mathrm{dt}$, stroke volume and ejection fraction increased (figure 4). At the paced cycle length of $1200 \mathrm{~ms}$ also the diastolic properties, i.e. $-\mathrm{dP} / \mathrm{dt}$, tau and end-diastolic pressure were improved. When the heart rate was increased from 50 to 100-130 beats per minute, the end-diastolic volume decreased, while the end-systolic volume remained similar (figure 4).

\section{Discussion}

For many years it is known that chronic complete atrioventricular-block in dogs leads to biventricular hypertrophy. ${ }^{14-20}$ The development of biventricular hypertrophy in these dogs is accompanied by functional, structural, electrophysiological and neurohumoral changes. $15,17-21$ Whether the hypertrophied heart is able to maintain its contractile function, has been a matter of dispute; while some authors found the development of heart failure, ${ }^{14,16}$ others found a compensated cardiac function. ${ }^{15,17,18,20,21}$ Since a compensated baseline cardiac function does not necessarily indicate a compensated function during increased demands, this study has focused on biventricular contractile function during the chronotropic stress of ventricular pacing.

The results of the present study in dogs with chronic ( 6 weeks) complete atrioventricular-block, indicate for the first time that during baseline the ventricular performance of both ventricles is well compensated. In addition, we show an inversed, but not depressed, force-frequency relation of both ventricles, indicating no deterioration of cardiac performance.

\section{Acute transition from sinus rhythm to idioventricular rhythm}

Immediately after the creation of complete atrioventricular-block, the heart is not able to maintain its pump function. Although the heart increases its stroke volume, cardiac output declines at the slow idioventricular rate.

The decrease in heart rate is associated with an acute volume loading of both ventricles: like others ${ }^{14,15}$ we have found an increase in both the right and the left ventricular end-diastolic pressure after the creation of atrioventricular-block. This is supported by 
the pronounced increase in left ventricular end-diastolic volume that was found when the paced cycle length was increased from $460-600$ to $1200 \mathrm{~ms}$ at acute atrioventricularblock. Presumably, it is this increased volume loading that is responsible for development of biventricular hypertrophy found in the dog with chronic complete atrioventricularblock. $^{14,16-20,25}$

\section{Transition from acute to chronic complete atrioventricular-block}

Six weeks after the creation of complete atrioventricular-block, the heart seems adapted to the bradycardia with the cardiac output restored to sinus rhythm values. This is accomplished by an increase in stroke volume. From the pressure-volume relations, it can be concluded that the heart increases its stroke volume by increasing the ejection fraction.

When compared with acute atrioventricular-block, the heart seems to function at a higher inotropic state during bradycardia (idioventricular rhythm or a paced cycle length of 1200 or $1250 \mathrm{~ms}$ ). The peak ejection rate increases despite an increased afterload, the $+\mathrm{dP} / \mathrm{dt}$ increases by an approximately equal preload and increased afterload and the ejection fraction increases resulting in an increased stroke volume, all indicating an improved systolic left ventricular performance. Although preliminary, due to the limited number of dogs, also the diastolic properties, i.e. - $\mathrm{dP} / \mathrm{dt}$, tau and end-diastolic pressure do not suggest a depressed function.

\section{Frequency potentiation during acute and chronic complete atrioventricular-block} In the normal myocardium, increasing the frequency of contraction exerts a positive inotropic effect. ${ }^{2-10}$ We also found a positive force-frequency relation in both ventricles using the $\mathrm{dP} / \mathrm{dt}$ when testing a range of paced cycle lengths from 1250 to $575 \pm 25 \mathrm{~ms}$ at acute atrioventricular-block. When the heart was paced at a shorter cycle length, the $+\mathrm{LV}$ $\mathrm{dP} / \mathrm{dt}$ decreased, giving the left ventricular force-frequency relation a biphasic appearance. This effect is not present in the right ventricle. Since left ventricular end-diastolic pressure shows no significant change, this does not seem to be caused by a decreased filling of the left ventricle, but appears intrinsic to the left ventricle.

During chronic atrioventricular-block, the force-frequency relation of both the left and the right ventricle seems inversed when looking to the $\mathrm{dP} / \mathrm{dt}$. However, at that time the force-frequency relation is determined at a higher starting point: the $+\mathrm{dP} / \mathrm{dt}$, end-systolic pressure and cardiac output are higher at all paced frequencies and the end-diastolic pressure is lower, which indicates that the function of both ventricles has improved. 
During the pacing induced rate acceleration, both the left and the right ventricular end-diastolic pressure markedly decrease. This suggests that reduced diastolic cardiac filling underlies the inversed force-frequency relation. The large differences in left ventricular end-diastolic volume between the paced cycle length of 460-600 ms and paced cycle length $1200 \mathrm{~ms}$ with equal end-systolic volumes determined with the conductance catheter support this hypothesis.

For the left ventricle, our observations are in contrast to the observations in dogs with left ventricular hypertrophy due to aortic banding with normal baseline left ventricular function $^{12}$ and in patients with heart failure. ${ }^{7-10}$ No information regarding the force-frequency relation in the intact hypertrophied right ventricle is available from other animal models or the intact human heart in heart failure.

\section{Conclusions}

These data show that in our dog model an inversed, but not depressed, force-frequency relation in both the left and the right ventricle exists without evidence of heart failure. On the contrary, during chronic complete atrioventricular-block, the dogs have adequately improved the performance of both ventricles.

\section{Acknowledgments}

This study was financially supported by a grant from the Wynand N. Pon Foundation, Leusden, The Netherlands.

\section{References}

1. Bowditch HP, Uber die Eigenthamlichkeiten der Reizbarkeit, welche die Muskelfasern des Herzens zeigen. Verh K Sachs Ges Wiss Leipzig 1871; 23: 652-689.

2. Buckley NM, Penefsky $Z Y$, Litwak RS. Comparative force frequency relationship in human and other mammalian ventricular myocardium. Pflugers Arch 1972; 332; 259-270.

3. Anderson PAW, Rankin JS, Arentzen CE, Anderson EW, Johnson EA. Evaluation of the force-frequency relationship as a descriptor of the inotropic state of canine left ventricullar myocardium. Cimc Res 1976; 39: 832-839.

4. Gwathmey $\mathrm{KK}$, Slawsky MT, Hajiar RJ, Briggs GM, Morgan JP. Role of intracellular calcium handling in force-interval relationships of human ventricular myocardium.J Clin Invest 1990; 85: $1599-1613$.

5. Pidgeon J, Lab M, Seed A, Elzinga G, Papadoyannis D, Noble MIM. The contractile state of cat and dog heart in relation to the interval between beats. Circ Res 1980; 47:559-567.

6. LiK, Rouleau JL. Tension-frequency relationships in normal and cardiomyopathic dog and hamster myocardium. J Mol Cell Cardiol 1995; 27 : 1251-1261. 
7. Schwinger RHG, Böhm M, Müller-Ehmsen J, Uhlmann R, Schmidt U, Stäblein A, Uberfuhr P, Kreuzer E, Reichant B, Eissmer JJ, Erdmann E. Effect of inotropic stimulation on the negative forcefrequency relationship in the failing human heart. Circulation 1993; 88: 2267-2276.

8. Pieske B, Kretschmann B, Meyer M, Holubarsch C, Weirich J, Posical H, Minami K, Just H, Hasenfuss $\mathrm{G}$. Alterations in intracellular calcium handling associated with the inverse force-frequency relation in human dilated cardionyopathy. Circulation 1995; 92:1169-1178.

9. Hasenfuss $\mathrm{G}$, Holubarsch $\mathrm{C}$, Hermann $\mathrm{HP}$, Astheimer $\mathrm{K}$, Pieske B, Just $\mathrm{H}$. Influence of the force frequency relationship on haemodynamics and left ventricular function in patients with non-failing hearts and in patients with dilated cardiomyopathy. Eur Heart $J 1994 ; 15: 164-170$.

10. Feldman MD, Alderman JD, Aroesty $\mathrm{MM}$, Royal HD, Ferguson JJ, Owen RM, Grossman W, McKay RG. Depression of systolic and diastolic myocardial reserve during atrial pacing tachycarditi in patients with dilated cardiomyopathy. $J$ Clin Imest 1988; 82:1661-1669.

11. Schmidt U, Schwinger RH, Böhm M, Erdmann E. Alterations of the force-frequency relation depending on stages of heart failure in humans. Am J Cardiol 1994; 74: 1066-1068.

12. Fujii AM, Gelpi RJ, Mirsky I, Vatner SF. Systolic and diastolic dysfunction during atrial pacing in conscious dogs with left wentricular hypertrophy. Circ Res 1988; 62: 462-470.

13. Schmidt HD, Hoppe H, Müller KD. The effect of changes in cardiac frequency on left and right ventricular dP/At max at different contractile states of the myocardium. Eur $J$ Appl Physial 1979; 42: 183-198.

14. Brockman SK. Cardiodynamics of complete heart block. Am J Cardiol 1965; 16: 72-83.

15. Newman WH. Contractile state of hypertrophied left ventricle in long-standing volume overload. Am I Physiol 1978; 234: H88-H93.

16. Starzl TE, Gaertner RA, Baker RR. Acute complete heart block in dogs. Circulation 1955; 12: 8289.

17. Turina M, Bussman WD, Krayenbuhl HP. Contractility of the hypertrophied canine heart in chronic volume overload. Cardiovasc Res 1969; 3: 486-495.

18. Vos MA, Van der Zande J, Verduyn SC, de Groot SHM, Kulcsár A, Cleutjens A, Van Bilsen M, Daemen M, Wellens HJJ. Physiologic hypertrophy after creation of complete atrioventricular block (abstract). Eur Heart J 1996; 17: 549.

19. Verduyn SC, Vos MA, Van der Zande J, De Groot SHM, Allessie MA, Wellens HJJ. Biventricular hypertrophy facilitates the occurence of acquired torsade de pointes arrhythmias in dogs (abstract). Circulation 1995; 92: 1504-1505.

20. Wusten $B, F$ lameng $W$, Schaper $W$. Cardiac function in the chronically volume-averloaded canine heart. Basic Res Cardiol 1977; 72: 172-177.

21. de Groot SHM, Vos MA, Leunissen JDM, Schoenmakers M, Wellens HJJ. Adaptations to preserve cardiac function predispose the hypertrophied canine heart for delayed afterdepolarization-dependent arrhythmias. Submitted.

22. Scherlag BJ, Kosowsky BD, Damato $\mathrm{AN}$. Technique for ventricular pacing from the His bundle of the intact heart. J Appl Physial 1967; 22: 584-587.

23. Conway $J$, Lund-Johansen $P$. Thermodilution method for measuring cardiac output. Eur Hearl $J$ 1990; 11: I17-I20.

24. Schreuder JJ, van der Veen FH, van der Velde ET, Delahaye F, Alfieri O, Jegaden $\mathrm{O}$, Larusso R, Jansen JRC, van Ommen V, Finet G, Wellens HJJ. Beat-to-beat analysis of left ventricular pressurevolume relation and stroke volume by conductance catheter and aortic model flow in cardiomyoplasty patients. Circulation 1995; $91: 2010-2017$.

25. Wusten B, Flameng W, Winkler B, Schaper W. Role of cardiac contractility in hypertrophy from chronic volume loading . Cardiovasc Res 1977; 11: 132-140. 


\section{Chapter 8}

General discussion 


\subsection{Introduction}

For more than 15 years, our group has been studying ventricular arrhythmias based on the mechanism of triggered activity. ${ }^{1}$ Initially, the emphasis was on delayed afterdepolarizations, ${ }^{1-14}$ but later we also studied early afterdepolarization-dependent Torsade de Pointes arrhythmias. ${ }^{14-16}$

To study the ventricular arrhythmias without interference of conducted supraventricular beats, a complete atrioventricular-block model was used. Following the creation of complete atrioventricular-block, the first experiment was initially delayed for at least 2 weeks: 1) to allow the dogs to recover from the thoracotomy, 2) to allow the dogs to become adapted to bradycardia (compensation phase?) and 3) to be sure of the development of a stable idioventricular rhythm. After this time, the dogs tolerated bradycardia well and the inducibility of triggered ventricular arthythmias became very reproducible.

In conscious dogs, assumed delayed afterdepolarization-dependent sustained ventricular tachycardias were initially induced using programmed stimulation and ouabain. The characteristics of these arrhythmias were studied using programmed electrical stimulation and multiple anti-arrhythmic drugs. ${ }^{4-6,8,12-14}$ Although this method was useful to develop mechanism specific interventions, a more direct approach to identify delayed afterdepolarizations with more certainty remained desirable. For that purpose, monophasic action potential catheter recordings seemed to be the most logical approach. Therefore, we evaluated the use of the monophasic action potential technique for the identification and quantification of delayed afterdepolarizations and related triggered arrhythmias (chapters 2,3 and 4). To introduce the catheters, general anesthesia was required, but otherwise the model remained the same.

\subsection{Use and limitations of the diastolic slope of the monophasic action potential to identify delayed afterdepolarizations}

Our primary goal was to identify delayed afterdepolarizations as the mechanism underlying both the initiation and the perpetuation of ventricular arrhythmias. We demonstrated the importance of the diastolic slope during pacing (chapters 2 and 3 ) in relation to arrhythmia initiation, and during ventricular tachycardia (chapters 3 and 4) in relation to arrhythmia perpetuation and termination. 
In vitro, when delayed afterdepolarizations are induced by pacing and cardiac glycoside administration, an increase in the diastolic slope of the action potentials has been observed before the induction of triggered delayed afterdepolarizations. ${ }^{23}$ In line with this observation we showed, during circumstances suspect for the development of delayed afterdepolarizations, that the diastolic slope of the monophasic action potential at the end of a pacing train can be used as a marker for the inducibility of delayed afterdepolarizations and related triggered ectopic beats (chapters 2 and 3). By testing the effect of different cycle lengths and lidocaine on the diastolic slope, we found that the slope behaved concordant with the occurrence of ectopic activity: an increase in the slope at the end of pacing was associated with an increase in ectopic activity, while a decrease in the slope was associated with the inability to induce arrhythmias. This dynamic behavior of the diastolic slope was further evaluated by analyzing its behavior during the pacing train. It was found that during pacing the diastolic slope seen in the monophasic action potential recordings varies (dynamic behavior), which relates to the arrhythmogenic outcome post-pacing: induction of (multiple) ectopic beats when the slope is high and overdrive suppression when it is low.

When studying delayed afterdepolarizations it becomes clear that a complete delayed afterdepolarization is only visualized when it does not trigger a new action potential. During sustained triggered activity, the delayed afterdepolarization is only visible as a steep diastolic upslope. Therefore, we quantified the behavior of the diastolic slope during ventricular tachycardia before spontaneous or drug induced termination using lidocaine (chapter 3) and ryanodine (chapter 4). We found, both in the left and right ventricular monophasic action potential, that a decline in the slope was indicative of ventricular tachycardia termination. Therefore, it seems that both during pacing and during ventricular tachycardia, changes in the slope can be used to identify and quantify delayed afterdepolarization-dependent triggered arrhythmias. This can be a helpful tool to identify delayed afterdepolarizations as the cause for arrhythmias in the intact human heart.

In vivo documentation of delayed afterdepolarizations as the cause of arrhythmias has been limited. ${ }^{24-30}$ The registration of delayed afterdepolarizations using the monophasic action potential technique is not generally accepted, even after its use was validated by interventions that are extensively described for this purpose in vitro, i.e. pacing, catecholamines and cardiac glycosides. This has delayed the use of this technique for in vivo identification of delayed afterdepolarizations under pathological circumstances. In part, this is caused by methodological problems, including 1) the stability of the monophasic 
Heart-10-body-weight ratios (H/BW, mean $\pm s d)$ at different duration

of chronic complete AV block (AVB; in weeks)
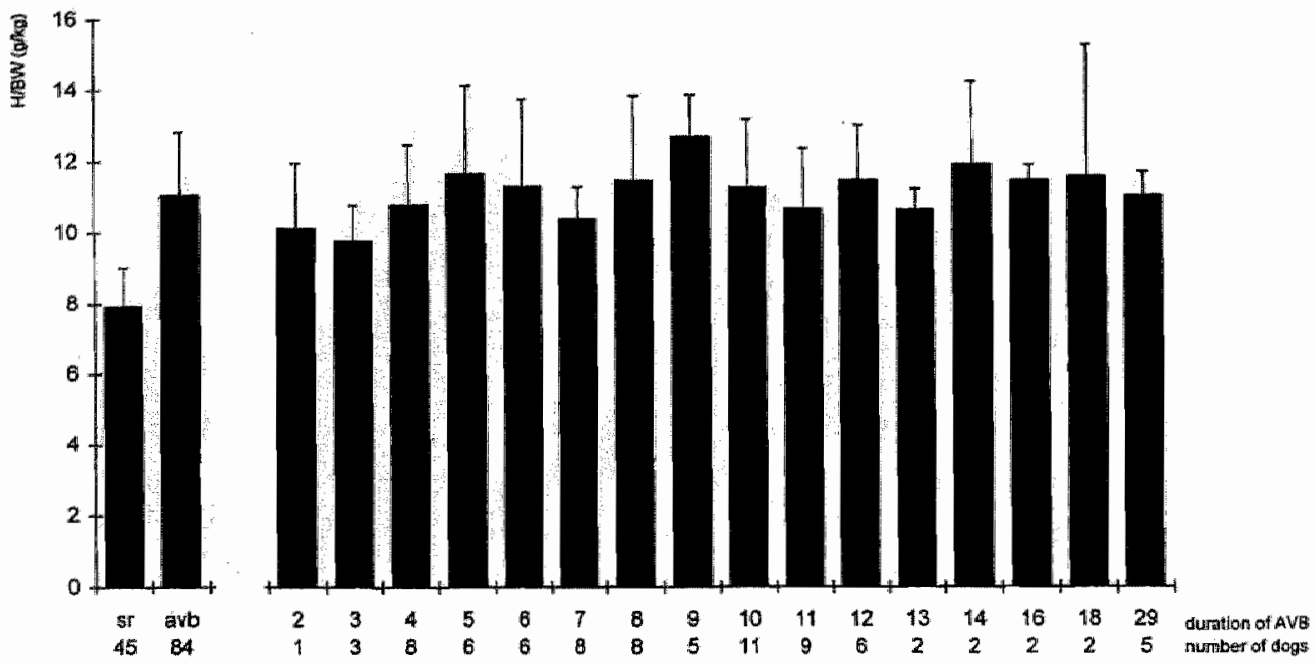

Figure 1: The effect of chronic complete atrioventricular-block (AVB) on heart to body-weight ratio (H/BW: $\mathrm{g} / \mathrm{kg}$ ).

At the left side of the bar chart the significant $(p<0.05)$ difference in $\mathrm{H} / \mathrm{BW}$ ratio (mean \pm sd) between dog with conducted sinus rhythm (SR) and chronic complete atrioventricular-block is indicated. At the right side the group atrioventricular-block dogs has been subdivided according to the different duration of complete atrioventricular-block. Below each bar, the duration of atrio-ventricular-block is given in weeks and the number of dogs represented in each bar is illustrated. This bar chart illustrates that H/BW ratio is stable after 2 weeks of atrio-ventricular-block.

action potential signal, 2) the possible registration of artifacts and 3) the proper selection of the recording site in relation to the site of afterdepolarization origin. To minimize the influence of these problems in registering afterdepolarizations, we have suggested criteria which, in our opinion, must be fulfilled to register afterdepolarizations correctly: 1) monophasic action potential-catheters should be placed by experts to record a stable monophasic action potential with the appropriate shape, 2) the equipment used to record and analyze monophasic action potential signals has to be of high quality, and 3) afterdepolarizations should be modifiable by interventions, i.e. pacing or drugs. At 
present, we have registered both early and delayed afterdepolarizations in the intact canine heart, using these criteria and we feel comfortable to use this technique under baseline circumstances and pathological as hypertrophy (chapter 6). To our knowledge, we are the first group able to show delayed afterdepolarizations reproducibly, provoked and suppressed with interventions, in the intact hypertrophied heart.

During our extensive use of the monophasic action potential, we have identified additional limitations of the monophasic action potential technique that need to be investigated. Firstly, with the monophasic action potential, we only study limited areas of the heart, which do not necessarily correspond with the site of origin of the afterdepolarizations or the arrhythmia and not necessarily reflect the whole myocardium. We therefore started using multiple monophasic action potential recordings to more accurately evaluate local differences in action potential duration and occurrence of afterdepolarizations. ${ }^{16}$ Secondly, we are still in the process of evaluating if the afterdepolarizations which we record are truly reflecting cellular events in underlying tissue or reflect electrotonic signals. Thirdly, the depth of the monophasic action potential registration is not known. Fourthly, when we used the diastolic slope of the monophasic action potential during pacing under non-drug circumstances, we noticed that the slope could not be calculated at long action potential durations. This problem was encountered when performing the experiments for chapter 6 , when it was not possible to quantify the diastolic slope at 6 weeks of atrioventricular-block during pacing in the left ventricular monophasic action potential. Finally, for reasons yet unknown, we are not able to obtain and quantify monophasic action potential signals in all dogs at all selected sites.

\subsection{Increased susceptibility to arrhythmias at chronic atrioventricular- block}

Despite the extensive use of dogs with chronic complete atrioventricular-block to study ventricular tachycardias, we were not informed about the adaptation processes after atrioventricular-block. All previous studies describing the consequences of complete atrioventricular-block in dogs reported the development of biventricular hypertrophy, ${ }^{17}$ 22 indicating the presence of such processes. Therefore, we wanted to get insight in the relation between the changes occurring after the creation of atrioventricular-block and the susceptibility to triggered arrhythmias (chapters 5,6 and 7). 


\section{Neurohumoral and structural adaptations}

In this thesis, we confirm that chronic complete atrioventricular-block leads to the development of biventricular hypertrophy as in severe volume overload (chapters 5 and 6). From 1989 to 1997 , we calculated the heart to body weight ratios for a total of 84 dogs, with a duration of complete atrioventricular-block that varied between 2 and 29 weeks (figure 1). When we compare the heart to body weight ratios for the different dogs at different durations of complete atrioventricular-block, we do not find differences in heart weight after the first 2 weeks (figure 1). During the period from the creation of atrioventricular-block to the day of sacrifice or demise, the body weight of the dogs remained similar.

In contrast to the observations made in pressure overload hypertrophy, we found normal collagen volume fraction and capillary fiber ratios in the myocardium, which suggests a balanced hypertrophy process with parallel increases in muscle, connective tissue and vessels (chapter 5). Also the lack of expression of ventricular mRNA ANF during chronic atrioventricular-block and the transient increase in plasma neurohumoral levels seem to indicate that the hypertrophy process is generally balanced. This is in marked contrast to the observations in canine models of congestive heart failure, in which all changes show a progressive nature. ${ }^{31-36}$ Of course, not all dogs behaved similarly. In the studied population, 1 dog showed clear physical symptoms of congestive heart failure. In this dog an increased collagen volume fraction, higher angiotensin II and aldosterone levels were found, together with right ventricular mRNA ANF expression (chapter 5, figure 7). This suggests that structural and neurohumoral derangements can occur, when cardiac function cannot be maintained. Consequently, the adaptations that occur in the majority of the dogs suggest an adaptive or physiological hypertrophy process, although it is initiated by an unphysiological action.

\section{Functional adaptations}

\section{Adaptations in steady state function}

To obtain insight in the functional adaptation of the dogs, we used several approaches. Initially we started using the left ventricular pressure signal during steady state spontaneous or paced rhythms (chapters 5 and 6 ). Later these experiments were extended with right ventricular pressure measurements, cardiac output measurements and in a limited number of dogs with pressure-volume relations (chapter 7). At all times, the dogs were 
serially tested before and immediately after the creation of permanent complete atrioventricular-block and 6 weeks later. In view of the plasma neurohumoral levels and the time dependency of the hypertrophy process this seemed the appropriate time for restudy (chapter 5, figure 4).

Immediately after the creation of atrioventricular-block, we found increased end-diastolic pressures, corresponding with increased volume loading of the heart, which was accompanied by an acute deterioration of cardiac pump function (chapter 7). After 6 weeks pump function was improved and the dogs had completely adapted, having an optimal pump function at the slow idioventricular rhythm. During chronotropic stress, the dogs were able to improve their pump function at both times. This observation is in marked contrast to the observations in dogs with aortic bandage and normal baseline left ventricular function. ${ }^{37}$ We only tested the heart during relative short periods of time and do not know the long term effect of relatively rapid heart rates in the hypertrophied hearts. It remains to be determined how the heart reacts to a dramatic alteration in heart rate for the second time (towards sinus rhythm frequency). At present it is under investigation whether this is accompanied by complete regression of hypertrophy with preservation of cardiac function (NHS 95.043, F. Prinsen and M. Peschar)

\section{Adaptations in dynamic function}

To get more insight in the dynamic function of the hypertrophied heart, which has been suggested to be representative for alterations in excitation-contraction coupling, ${ }^{38-41}$ we determined the post-extra-systolic potentiation by interposing a single extrastimulus (with variable coupling interval) during fixed rate pacing (chapter 5). This seemed a logical approach, since we had always used pacing to induce delayed afterdepolarization-related ectopic beats with the idea that they would occur after reaching sufficiently high levels of intracellular calcium. Like in delayed afterdepolarizations, the potentiation of the post-extrasystolic beat has been equated to calcium. More specifically, to the extra amount of calcium, made available by the extrasystoles. ${ }^{38-41}$ We had expected to induce delayed afterdepolarization-related ectopic activity with this post-extrasystolic potentiation protocol, but this turned it out not to be the case. Therefore, we extendled the number of interposed stimuli, in an attempt to increase calcium loading and thereby to increase either the post-pacing potentiation or the likelihood to induce ectopic activity (chapter 6). 
We were able to demonstrate that the hypertrophied left ventricle is still able to potentiate both after one or multiple extrastimuli and that the decline of potentiation (relaxation) is similar at acute and chronic complete atrioventricular-block, suggesting that the left ventricular sarcoplasmic-reticulum function is maintained in our dogs at the time tested. Also we were able to induce delayed afterdepolarization-dependent triggered activity with the multiple extrastimuli protocol, suggesting alterations in intracellular calcium handling in the dogs with chronic complete atrioventricular-block.

In collaboration with the Department of Physiology of the Free University of Amsterdam (NHS M 93008, C. van Hardeveld, A. Muller and M. Zuidweg) we attempted to obtain additional information regarding expression of calcium-regulating genes and proteins. In part due to large interindividual variations, possibly caused by differences in race and duration of atrioventricular-block, results have been confusing. Future experiments, using serial biopsies from individual dogs will be needed.

Relation between the relative increase in APD and $\mathrm{dP} / \mathrm{dt}$ in acute and chronic complete AV-block, in the $\operatorname{RV}(0)$ and $\operatorname{LV}(\bullet)$

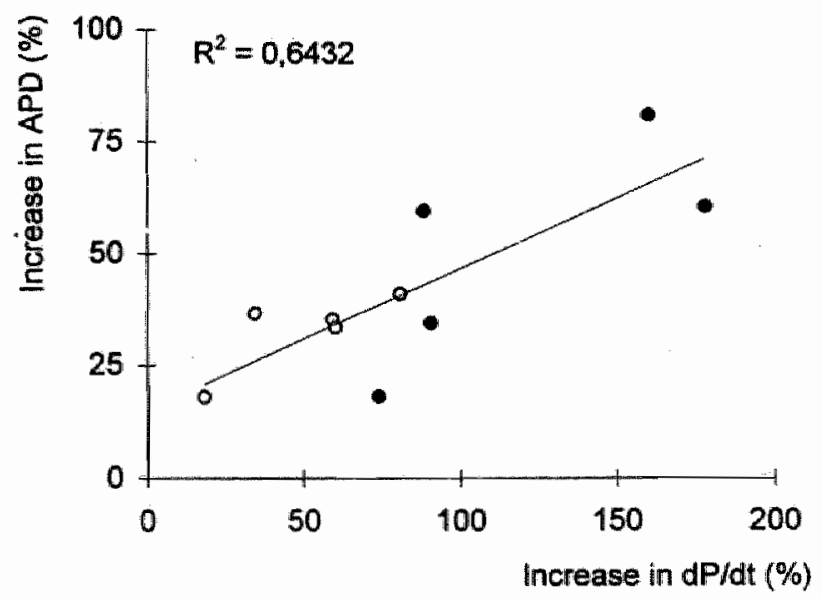

Figure 2: Graph illustrating the relation between the relative increase in action potential duration (APD) and positive $\mathrm{dP} / \mathrm{dt}$ in acute and chronic complete atrio-ventricular-block in the right and the left ventricle (RV respectively LV).

At the vertical horizontal axis, the relative increase in APD from acute to chronic (6 weeks) atrioventricularblock and at the horizontal axis the relative increase in $\mathrm{dP} / \mathrm{dt}$ in this period. It is illustrated that the increase in $\mathrm{dP} / \mathrm{dt}$ relates to the APD prolongation. The relative increase in $\mathrm{dP} / \mathrm{dt}$ is smaller in the $\mathrm{RV}$ when compared to the LV, which corresponds with less APD prolongation in the RV. 
Another way to more conclusively study the role of intracellular calcium (dys)handling in the relation between functional adaptations and triggered arrhythmias could be provided by cellular electrophysiological and functional studies (K.R. Sipido and P.G.A. Volders).

\section{Electrophysiological adaptations}

In contrast to functional, structural and neurohumoral adaptations, the electrophysiological alterations caused by hypertrophy have been less extensively described in the different canine models. ${ }^{42-47}$ The only systematic finding is the prolongation of the repolarization, both in vivo (monophasic action potential recordings and QT) and in

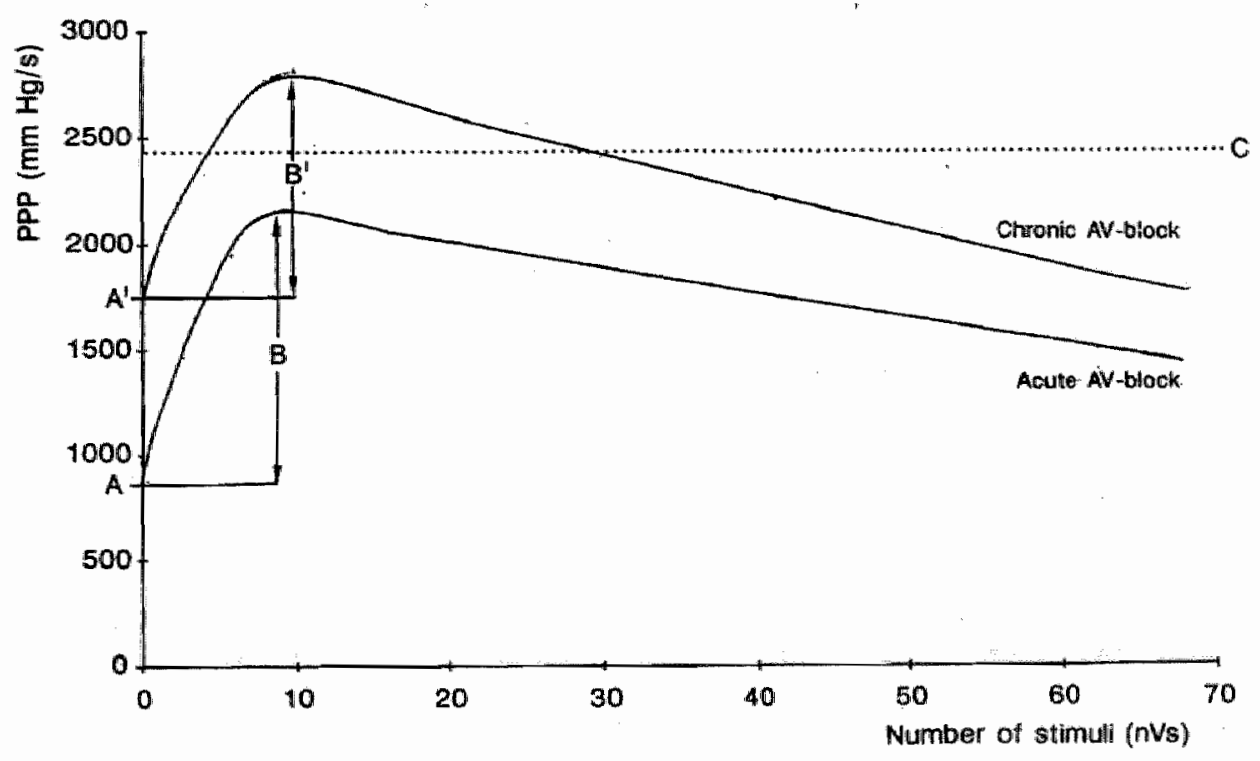

Figure 3: Hypothetical graph illustrating the possible ways by which pacing with different number of stimuli leads to the highest post-pacing potentiation (PPP: $\mathrm{mm} \mathrm{Hg/s}$ ) and to the induction of triggered arrhythmias at chronic complete atrio-ventricular-block.

When the amount of post-pacing potentiation reaches a certain level, an arrhythmogenic window occurs. This level is illustrated by the $C$. This level can be reached by a certain baseline inotropic state (indicated by the A) or by a certain amount of potentiation (indicated by the B). At chronic complete atrioventricular-block the baseline inotropic state has increased from $A$ to $A$; while the amount of potentiation has decreased from $B$ to $B$ '. This results in the frequent occurrence of arrhythmias at chronic atrioventricular-block, suggesting an important role for the baseline inotropic state in the genesis of these arrhythmias. The rolle of the level of potentiation reached and its variation after atrioventricular-block remains to be determined. 
vitro. ${ }^{44,46,47}$ Also in our atrioventricular-block model, we found a pronounced action potential prolongation of $32 \%$ in the left ventricle and $21 \%$ in the right ventricle (chapter 5). This increase in action potential duration is more marked than in other models of left ventricular hypertrophy, which could be caused by a mutual effect of both hypertrophy and bradycardia. Possibly, bradycardia alone could be responsible for altered electrophysiological properties of the myocardium as has been observed previously in tachycardia-induced electrical remodeling. ${ }^{48}$ Future experiments will be directed to distinguish between the consequences of hypertrophy and of bradycardia.

That the degree of left ventricular action potential prolongation exceeds that of the right ventricle, while hypertrophy was largest in the right ventricle, was a remarkable observation. Apparently, action potential duration prolongation is determined by more than hypertrophy and/or bradycardia alone. A possible explanation could be a difference in rate response of the left and right ventricle, which seems supported by the more pronounced increase in left than right ventricular action potential duration at longer cycle lengths. ${ }^{49}$ Another possibility could be a difference in adaptation to volume overload between both ventricles. In retrospect, we evaluated the relative increase in left and right ventricular $\mathrm{dP} / \mathrm{dt}$ between acute and chronic ( 6 weeks of) complete atrioventricular-block and related this to the relative increase in action potential duration in the left and right ventricle in 5 dogs, from whom we had these data available (figure 2). Although preliminary, the average increase in left ventricular $\mathrm{dP} / \mathrm{dt}$ is larger than the increase in right ventricular $\mathrm{dP} / \mathrm{dt}$, which corresponds with a larger increase in left than right ventricular action potential duration. There was no correlation between the right or left ventricular action potential duration and the cycle length of the idioventricular rhythm. This analysis suggests that the larger augmentation in ventricular function, the larger the increase in action potential duration. However, the true mechanisms underlying the difference in action potential duration response remain to be determined.

Due to the relatively smaller action potential prolongation in the right ventricle when compared to the left ventricle, the interventricular dispersion is increased after 5 weeks of atrioventricular-block (chapter 5). After administration of $d$-sotalol this interventricular action potential duration dispersion increased further, resulting in increased inducibility of Torsade de Pointes arrhythmias. A large interventricular dispersion is one of the prerequisites for the induction of these arrhythmias in our animal model. ${ }^{16}$ In addition to interventricular dispersion, the initiation of Torsade de Pointes arrhythmias has been related to triggered activity and early afterdepolarizations. 
Besides an increased susceptibility to class-III-induced early afterdepolarization-related Torsade de Pointes arrhythmias, the dogs with chronic complete atrioventricular-block also exhibited an increased propensity to delayed afterdepolarizations and related triggered ectopic beats, when the heart was provoked by protocols using short lasting pacing (chapter 6). Although an increased propensity to develop delayed afterdepolarizationdependent triggered activity in hypertrophied myocardium has been described in vitro, ${ }^{50-}$ 54 we are the first to report this in vivo. The increased sensitivity to delayed afterdepolarizations in the hypertrophied heart could be related to adaptations in intracellular calcium handling, but could also relate to neurohumoral changes at the level of the myocardium.

\subsection{Relation between potentiation, delayed afterdepolarizations and triggered arrhythmias}

During short lasting pacing protocols, we found that both the amount of potentiation and the inducibility of delayed afterdepolarization-related ectopic beats relate similarly to an increase in number of stimuli. This resulted in a maximal post-pacing potentiation and a maximal inducibility after 5-13 stimuli (chapter 6, figure 1). However, ectopic beats are only induced at 6 weeks of complete atrioventricular-block, when the level of post-pacing potentiation is highest. Further increasing the amount of post-pacing potentiation with therapeutic dosages of ouabain increases the inducibility of ectopic beats at 6 weeks of atrioventricular-block, but not at acute atrioventricular-block. This suggests the existence of an arrhythmogenic window at a certain level of potentiation. In figure 3 , we have hypothesized how increasing potentiation can lead to a temporarily present arrhythmogenic window. Either the baseline inotropic state (from $A$ to $A^{\prime}$ ), or the absolute increase in potentiation (indicated by $\mathrm{B}$ and $\mathrm{B}^{\prime}$ ) can be increased, or both can increase. In addition, the level of potentiation at which arrhythmias will occur (indicated by C) may vary. At present, we cannot indicate the exact level at which ectopic beats will occur. Since the absolute amount of potentiation decreases from acute to chronic atrioventricular-block (indicated by $B$ and $B^{\prime}$ ), it seems that the increase in baseline ventricular performance at 6 weeks of atrioventricular-block is at least in part responsible for the predisposition to arrhythmias. However, even when provoked by ouabain, pacing (rate changes?) is necessary to reveal the altered arrhythmia propensity. 


\subsection{Implications of the predisposition to triggered arrhythmias}

The increased propensity of the hypertrophied heart to develop ventricular arrhythmias, has often been related to pathophysiological conditions like 1) fibrosis, 2) ischemia, 3) regional differences in the degree and nature of hypertrophy and 4) functional decompensation of the heart. ${ }^{55,56}$ It is important to emphasize that in the dog with chronic complete atrioventricular-block, the predisposition to develop triggered arrhythmias, whether early- or delayed afterdepolarization-related, seems to occur in hypertrophied myocardium in the absence of additional pathological conditions. In contrast, the arrhythmias occur in dogs with an improved cardiac function. Whether similar arrhythmias can be induced or occur in patients with left ventricular hypertrophy remains to be determined.

All arrhythmias described in this thesis were provoked by ventricular pacing (sometimes in combination with drugs) in anesthetized dogs. Using Holter recordings and telemetry we observed spontaneously accurring arrhythmias in the conscious dog with chronic complete atrioventricular-block. Additionally, we noticed that approximately $10 \%$ of our atrioventricular-block dogs died suddenly in their stable, not related to an experiment in the preceding 24 hours. How the increased propensity to develop ectopic activity and arrhythmias during anesthesia relates to the arrhythmias and/or sudden death occurring in conscious dogs, will be the topic of future research.

\section{References}

1. Gorgels AP, Beekman HD, Brugada P, Dassen WR, Richards DA, Wellens HJ. Extrastimulus-related shortening of the first postpacing interval in digitalis-induced ventricular tachycardia: Observations during programmed electrical stimulation in the conscious dog. $t$ Am Coll Cardiol 1983; 1 : $840-857$.

2. Gorgels APM, de Wit B, Beekman HDM, Dassen WR, Wellens HJJ. Triggered activity induced by pacing during digitalis intoxication observations during programmed electrical stimulation in the conscious dog with chronic complete atrioventricular block. PACE 1987; 10: 1309-1321.

3. Gorgels APM, de Wit B, Beekman HDM, Dassen WRM, Wellens HJJ. Effects of different modes of stimulation on the morphology of the first QRS-complex following pacing during digitalis induced ventricular tachycardia. Observations in the conscious dogs with chronic complete atrioventricular block. PACE 1986; 9: 842-859.

4. Vos MA, Fazekas T, Gorgels AP, Leunissen JD, Wellens $\mathrm{H}$. Action of MgSO4 differs from moricizine and verapamil on ouabain-induced ventricular tachycardia in normomagnesemic conscious dogs. J Cardiovasc Pharmacol 1994; 23: 252-258.

5. Vos MA, Gorgels AP, Leunissen-Beekman JD, Brugada P, Wellens HJ. The effect of an entrainment protocol on ouabain-induced ventricular tachycardia. PACE 1989; 12:1485-1493. 
6. Vos MA, Gorgels AP, Drenth JP, Leunissen JD, Wellens HJ. Termination of ouabain-induced ventricular tachycardia by flunarizine in conscious dogs. Eur $J$ Pharmacol 1989; 165: 139-145.

7. Vos MA, Gorgels AP, de Wit B, Drenth IP, van Deursen RT, Leunissen JD, Wellens HJ. Premature escape beats. A model for triggered activity in the intact heart? Circulation 1990;82:213-224.

8. Vos MA, Gorgels AP, Leunissen JD, Wellens HJ. Flunarizine allows differentiation between mechanisms of arrhythmias in the intact heart. Circulation 1990; 81:343-349.

9. Vos MA, Gorgels AP, Leunissen JD, Wellens. HJ. The in vivo response of ouabain-induced arrhythmias to pacing acceleration instead of termination. Am Heart $J 1990 ; 120: 604-611$.

10. Vos MA., Gorgels APM, Leunissen JD, van Deursen RT, Wellens HJJ. Significance of the number of stimuli to initiate ouabain-induced arrhythmias in the intact heart. Circ Res 1991; 68; 38-44.

11. Vos MA, Gorgels APM, Leunissen JD, van der Nagel T, Halbertsma FJ, Wellens HJJ Further observations to confirm the arrhythmia mechanism-specific effects of flunarizine $J$ Cardiovasc Pharmacol 1992; 19: 682-690.

12. Verduyn SC, Vos MA, van der Zande J, Kulscàr A, Wellens HJJ. Further observations to elucidate the role of interventricular dispersion of repolarization and early afterdepolarizations in the genesis of acquired Torsade de Pointes arrhythmias. A comparison between almokalant and $d$-sotolol using the dog as its own control. J Am Coll Cardiol 1997; 30: 1575-1584.

13. van Deursen RT, Gorgels AP, Leunissen JD, Wellens HJ. R56865, an antiarrhythmic drug with class III effects that terminates ouabain induced ventricular tachycardia in an inverse rate dependent manner. Cardiovase Res 1993; 27: 1491-1497.

14. Vos MA, Gorgels AP, Lipcsei GC, De Groot SH, Leunissen JD, Wellens HJ. Mechanism-specific antiarrhythmic effects of the potassium channel activator levcromakalini against repolarization-dependent tachycardias. J Cardiovasc Electrophysiol 1994; 5: 731-742.

15. Vos MA, Verduyn SC, Gorgels APM, Lipesei GC, Wellens HJJ. Reproducible induction of early afterdepolarizations and torsade de pointes arrhythmias by d-sotalol and pacing in dogs with chronic atrioventricular block. Circulation 1995; 91 : 864-872.

16. Verduyn SC, Vos MA, van der Zande J, van der Hulst FF, Wellens HJJ. Role of interventricular dispersion of repolarization in acquired Torsade de Pointes arrhythmias. reversal by magnesium. Cardiovasc Res 1997; 34: 453-463.

17. Brockman SK. Cardiodynamics of complete heart block. Am J Cardiol 1965; 16: 72-83.

18. Newman WH. Contractile state of hypertrophied left ventricle in long-standing volume overload. Am J Physiol 1978; 234: H88-H93.

19. Starzl TE, Gaertner RA, Baker RR. Acute complete heart block in dogs. Circulation 1955; 12: 82 89.

20. Turina $M_{3}$ Bussman WD, Krayenbuhl HP. Contractility of the hypertrophied canine heart in chromic volume overload. Cardiovasc Res $1969 ; 3: 486-495$.

21. Wusten B, Flameng W, Winkler B, Schaper W. Role of cardiac contractility in hypertrophy firom chronic volume loading. Cardiovasc Res 1977; 11: 132-140.

22. Wusten $B$, Flameng W, Schaper W. Cardiac function in the chronically volume-owerloaded canine heart. Basic Res Cordiol 1977; 72: 172-177.

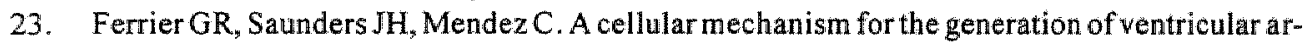
rhythmias by acetylstrophanthidin. Circ Res 1973;32:600-609.

24. Patterson E, Szabo B, Scherlag BJ, Lazzara R. Early and delayed afterdepolarizations associated with cesium chloride-induced arrhythmias in the dog. JCardiovasc Pharmacol 1990; 15:323-331.

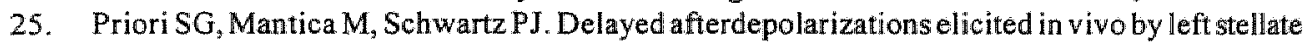
ganglion stimulation. Circulation 1988; 78: 178-185. 
26. Furukawa $T$, Kimura S, Castellanos A, Bassett AL, Myerburg RI. In vivo induction of "focal" triggered wentricular arthythmias and responses to overdrive pacing in the canine heart. Circulation $1990 ; 82: 549-559$.

27. Chen SA, Chiang CE, Yang CJ, Cheng CC, Wu TJ, Wang SP, Chiang BN, Chang MS. Sustained atrial tachycardia in adult patients. Electrophysiological characteristics, pharmacological response, possible mechanisms, and effects of radiofrequency ablation. Circulation 1994;90: 1262-1278.

28. Xu J, Hurt CM, Pelleg A. Digoxin-induced ventricular arthythmias in the guinea pig heart in vivo: Evidence for a role of endogenous catecholamines in the genesis of delayed afterdepolarizations and triggered activity. Heart and Vessels 1995; 10:119-127.

29. Xu J, Pelleg A. A novel guinea pig heart model for studying atrioventricular-nodal conduction and triggered activity in vivo. Am I Physiol 1996; 270: H1850-H1857.

30. Xu J Zaim $\mathbf{S}$, Pelleg A. Effects of pinacidil, verapamil, and heart rate on afterdepolarizations in the guinea-pig heart in vivo. Heart and Vessels 1996; 11: 289-302.

31. Sabbah HN, Sharov VG, Lesch $M$, Goldstein S. Progression of heart failure: A role for interstitial fibrosis. Mol Cell Biochem 1995; 147: 29-34.

32. Sabbah HN, Stein PD, Kono T, Gheorghiade M, Levine TB, Jafri S, Hawkins ET, Goldstein S. A canine model of chronic heart failure produced by multiple sequential coronary microem bolizations. Am J Physiol 1991; 260 : H1379-H1384.

33. Hasebe N, Hittinger L, Kohin $S$, Shen YT, Graham RM, Vatner SF. Effects of hypertrophy on left atrial and ventricular compliance and plasma ANF levels in conscious dogs. Am J Physiol 1995; 268: $\mathrm{H} 781-787$.

34. Spinale FG, Holzgreve HH, Mukherjee R, Arthur SR, Child MJ, Powell JR, Koster WH. Left ventricular and myocyte function after early recovery from tachycardia induced cardiomyopathy. $A m$ J Physiol 1995; 268: H836-H847.

35. Weber KT, Pick R, Silver MA, Moe GW, Janicki JS, Zucker IH, Amstrong PW. Fibrillar collagen and remodeling of the dilated canine left ventricle. Circulation 1990;82:1387-1401.

36. Moe GW, Stopps TP, Angus C, Forster C, De Bold AJ, Amstrong PW. Alterations in serum sodium in relation to atrial natriuretic factor and other neuroendocrine variables in experimental pacing-induced heart failure. $J$ Am Coll Cardiol 1989; 13: 173-179.

37. Fujii AM, Gelpi RJ, Mirsky I, Vatner SF. Systolic and diastolic dysfunction during atrial pacing in conscious dogs with left ventricular hypertrophy. Circ Res 1988; 62: 462-470.

38. Vatner DE, Sato N, Kiuchi K, Shannon RP, Vatner SF. Decrease in myocardial ryanodine receptors and altered excitation-contraction coupling early in the development of heart failure. Circulation $1994: 90: 1423-1430$.

39. ter Keurs HEDI, Schouten VJA, Bucx JJ, Mulder BM, de Tombe PP. Excitation-contraction coupling in myocardium: implications of calcium release and $\mathrm{Na}^{+}-\mathrm{Ca}^{2+}$ exchange. Can $J$ Physiol 1987 ; 65: $619-626$.

40. Schouten VJA, Vliegen HW, van der Laarse A, Huysmans HA. Altered calcium hand ling at normal contractility in hypertrophied rat heart. $J$ Mol Cell Cardiol 1990; 22: 987-998.

41. Cooper MW. Postextrasystolic potentiation: do we really know what it means and how to use it? Circulation 1993; 88: 2962-2971.

42. LiHG, Jones DL, Yee R, Klein GJ. Electrophysiologic substrate associated with pacing-induced heart failure in dogs: potential value of programmed stimulation in predicting sudden death. $J \mathrm{Am}$ Coll Cardiol 1992; 444-449.

43. LiHG, Jones DL, Yee R, Klein GJ. Arrhythmogenic effects of catecholamines are decreased in heart failure induced by rapid pacing in dogs. Am J Physial 1993; 265: H1654-H1662. 
44. Pak PH, Nuss HB, Tunin RS, Kääb S, Tomaselli GF, Marban E, Kass DA. Repolarization abnormalities, arrhythmia and sudden death in canine tachycardia-induced cardiomyopathy. IAm Coll Cardiol 1997; 30: 576-584.

45. Sabbah HN, Goldberg DG, Schoels W, Kono T, Webb C, Brachmann J Goldstein S. Spontaneous and inducible ventricular arrhythmias in a canine model of chronic heart failure: relation to haemodynamics and sympathoadrenergic activation. Eur Heart J 1992; 13: 1562-1572.

46. Ben-David J, Zipes DP, Ayers GM, Pride HP. Canine left ventricular hypertrophy predisposes to ventricular tachycardia induction by phase 2 early afterdepolarizations after administration of BAY K 8644. J Am Coll Cardiol 1992; 20: 1576-1584.

47. Kääb S, Nuss HB, Chaimvimonvat N, O'Rourke B, Pak PH, Kass DA, Marban E, Tomaselli GF. Ionic mechanism of action potential prolongation in ventricular myocytes from dogs with pacinginduced heart failure. Circ Res 1996; 78: 262-273.

48. Wijffels MCEF. Atrial fibrillation begets atrial fibrillation. An experimental study in chronically instrumented goats. Thesis Maastricht University, 1996.

49. Di Diego JM, Sun Z-Q, Antzelevitch C. Ito and the action potential notch are smaller in left versus right canine ventricular epicardium. Am J Physiol 1996; 271 : H548-H561.

50. Vermeulen JT, McGuire MA, Opthof T, Coronel R, de Bakker JM, Klopping C, Janse MJ. Triggered activity and automaticity in ventricular trabeculae of failing human and rabbit hearts. Cardiovase Res $1994 ; 28: 1547-1554$.

51. Aronson RS. Afterpotentials and triggered activity in hypertrophied myocardium from rats with renal hypertension. Circ Res 1981; 48: 720-727.

52. Aronson RS, Ming Z Cellular mechanisms of arrhythmias in the hypertrophied and failing myocardium. Circulation 1993; 87: VII76-VII83.

53. Charpentier $\mathrm{F}$, Baudet $\mathrm{S}$, Le Marec $\mathrm{H}$. Triggered activity as a possible mechanism for arrhythmias in ventricular hypertrophy. PACE 1991; 14:1735-1741.

54. Samson RA, Lee H-C. Delayed afterdepolarizations and triggered arrhythmias in hypertrophic cardiomyopathic hearts. JLab Clin Med 1994; 124: 242-248.

55. Gorgels AP, Vos MA, Smeets JL, Wellens HJ. Ventricular arrhythmias in heart failure $A, m J$ Cardial 1992; 70: 37C-43C.

56. Assayag P, Carré F, Chevalier B, Delcayre C, Mansier P, Swynghedauw B. Compensated cardiac hypertrophy: arrhythmogen icity and the new myocardial phenotype. I. Fibrosis. Cardiovasc Res 1997; 34: 439-444. 


\section{Summary}

Cardiac hypertrophy is the adaptive mechanism of the heart to mechanical overload of any cause, including hypertension, myocardial infarction and valvular heart disease. The prevalence of left ventricular hypertrophy in the total adult population is $10-20 \%$ and increases with age. Left ventricular hypertrophy has been associated with an increased susceptibility to ventricular arrhythmias and sudden arrhythmic death. This risk seems to exceed the risk attributable to coexisting arrhythmia-promoting factors, like ischemia or congestive failure. However, the exact relation between ventricular arrhythmias, sudden death and functional status of the heart has not been studied properly. Insight in the different disease stages (compensated or decompensated hypertrophy) together with a better understanding of the arrhythmogenic mechanisms involved, could be important to develop appropriate therapies and to determine the timing of the interventions. To unravel the complex nature of the disease process, animal models of hypertrophy, with or without subsequent development of heart failure could provide useful alternatives to clinical studies. In this thesis, the susceptibility of the hypertrophied canine heart to triggered ventricular arthythmias based on early or delayed afterdepolarizations is studied and related to histological and functional adaptations which accompany the hypertrophy process.

For this purpose, cardiac hypertrophy is induced in adult mongrel dogs through the creation of chronic complete atrioventricular-block. By the injection of a small amount of formaldehyde in the atrioventricular-node area, the conduction between the atria and the ventricles is interrupted permanently. This results in a pronounced slowing of the heart rate, which is accompanied by increased volume loading of the ventricles. Most likely, this volume overloading of the ventricles triggers the development of biventricular hypertrophy.

In chapter 1 a review of the literature is given. This review describes the clinical background of the thesis and the possible arrhythmogenic mechanisms that could be involved in (de)compensated hypertrophy. In addition, the different canine models of hypertrophy and/or heart failure are reviewed. Until now, the majority of these models have not been developed to study arrhythmogenesis in hypertrophy. Therefore, no studies are available to relate arrhythmogenesis to functional (de)adaptations.

To be able to identify and quantify delayed afterdepolarizations as the mechanism underlying both the initiation and the perpetuation of ventricular arrhythmias in the intact heart, we evaluated the use of the monophasic action potential (chapters 2,3 and 4). In 
circumstances suspect for the development of delayed afterdepolarizations (ouabain intoxication), it was demonstrated that the diastolic slope of the monophasic action potential recording during ventricular pacing can be used as a marker for the ability to induce delayed afterdepolarizations and related ventricular arrhythmias (chapters 2 and 3). Using different durations of pacing, it was found that the diastolic slope of the monophasic action potential recording varies during pacing, which relates to the arrhythmogenic outcome post-pacing: induction of (multiple) ectopic beats when the slope is high and overdrive suppression when it is low. Similarly, during ouabain induced ventricular tachycardia, a decline in the diastolic slope of the monophasic action potential recording was indicative for termination of the arrhythmia (chapters 3 and 4 ).

After the validation of the monophasic action potential catheter to register afterdepolarizations, this technique was applied in the hypertrophied heart to evaluate its susceptibility to triggered arrhythmias (chapters 5 and 6). In addition, the electrophysiological, histological and functional adaptation processes that occur after the creation of atrioventricular-block were studied (chapters 5,6 and 7). Using group comparisons, the results of this thesis confirm that chronic (more than 2 weeks) complete atrioventricular-block results in biventricular hypertrophy. The type of hypertrophy that occurs is characterized by normal interstitial fibrosis and normal capillary fiber ratios, which suggests a balanced hypertrophy process with parallel increases in muscle, connective tissue and vessels (chapter 5). In contrast to other canine models of cardiac hypertrophy, the dogs with complete atrioventricular-block showed no physical signs of congestive heart failure. This is in agreement with a transient increase in plasma neurohormones and the absence of mRNA ANF expression in both the hypertrophied left and right ventricle. During steady state, idioventricular or paced rhythms, cardiac function improved when acute atrioventricular-block was compared with 6 weeks of chronic complete block. (chapter 7). Also the dynamic function of the hypertrophied left ventricle, tested by post-extrasystolic potentiation or post-stimulation potentiation, remained normal after 6 weeks of complete atrioventricular-block (chapters 5 and 6).

Although the histological, neurohumoral and functional adaptations indicate adequate cardiac function, the heart with chronic complete atrioventricular-block is predisposed for acquired Torsade de Pointes arrhythmias (chapter 5) and delayed afterdepolarization-dependent triggered arrhythmias (chapter 6). The increased susceptibility to acquired Torsade de Pointes arrhythmias seems related to a non-uniform prolongation of the action potential duration and changed sensitivity for $d$-sotalol, while the occurrence 
of delayed afterdepolarization-dependent triggered arrhythmias is related to an increased contractile state of the heart.

Finally, our findings and relevant information from the literature are discussed in chapter 8. 


\section{Samenvatting}

Het hart is een holle spier die in rust ongeveer 70 keer per minuut samentrekt om bloed. door het lichaam te pompen. De functie van het hart kan worden bemoeilijkt door een te hoge bloeddruk, een lekkende of vernauwde hartklep, of door een hartspierbeschadiging na een hartinfarct. Om onder deze omstandigheden toch voldoende bloed door het lichaam te pompen, neemt de spiermassa van het hart toe (hypertrofie). Soms kan het hart hierdoor zijn pompfunctie handhaven en spreken wij van een "gecompenseerde" functie. In andere gevallen is de pompfunctie van het hart, in rust of tijdens inspanning, onvoldoende of gedecompenseerd en is er sprake van hartfallen.

Ongeveer 10 tot $20 \%$ van de totale volwassen bevolking heeft een hypertroof hart. Dit gaat gepaard met een groter risico op het krijgen van kamerritmestoomissen en plotse hartdood. Het mechanisme dat hieraan ten grondslag ligt, is nog onbekend. Ook is het moment van optreden nog niet duidelijk: als de pompfunctie van het hart goed is, als deze heel slecht is, of in een hiertussen liggende overgangsfase. Om meer inzicht te verkrijgen in dit complexe ziekteproces kunnen diermodellen van cardiale hypertrofie, eventueel gecombineerd met hartfalen, gebruikt worden. In dit proefschrift is de gevoeligheid van het hypertrofe hondenhart voor het ontwikkelen van kamer-ritmestoornissen, gebaseerd op getriggerde activiteit, bestudeerd. Getriggerde activiteit kan het gevolg zijn van zowel vroege als late nadepolarizaties op basis van functionele en electrofysiologische veranderingen ten gevolge van de hypertrofie.

Om hypertrofie te induceren, wordt bij volwassen honden een blijvend compleet atrioventriculair geleidingsblok gemaakt. Dit resulteert in een vertraging van het hartritme, waardoor de hartkamers per slag een veel groter volume bloed moeten verplaatsen. Deze grotere volumebelasting veroorzaakt hypertrofie van beide hartkamers.

Naast de klinische reden van het proefschrift worden in hoofdstuk 1 de mechanismen die een rol spelen bij het ontstaan van ritmestoornissen in het hypertrofe hart besproken. Ook wordt een overzicht gegeven van de verschillende hondenmodellen die in gebruik zijn om kamerhypertrofie en/of hartfalen te induceren. In de meeste van deze modellen worden geen ritmestoornissen bestudeerd, zeker niet in relatie tot functionele (de)adaptaties.

Voor de identificatie van late nadepolarizaties als verantwoordelijk mechanisme voor zowel de initiatie als het in stand houden van ritmestoornissen, is de monofasische 
actie-potentiaal catheter gebruikt (hoofdstukken 2, 3 en 4). In de geanestheseerde hond werden met behulp van oubaine (een hartglycoside) en ventriculair pacen late nadepolarizaties opgewekt. De steilheid van de diastolische helling van het monofasische actie-potentiaal-signaal tijdens kortdurende stimulatie protocollen bleek bruikbaar als maat voor de induceerbaarheid van late nadepolarizaites en de daarop gebaseerde ritmestoornissen (hoofdstukken 2 en 3). Tijdens de stimulatieprotocollen varieerde de steilheid van de diastolische helling van het monofasische actie-potentiaal-signaal. Dit beînvloedde de induceerbaarheid van de ritmestoomissen: bij een hoge steilheid werd ectopische activiteit geïnduceerd, terwijl er geen ritmestoomissen geïnduceerd werden als de diastolische helling vlak was. Ook tijdens ventriculaire tachycardie bleek een afname van de steilheid van de diastolische helling indicatief voor terminatie van de ritmestoornis (hoofdstukken 3 en 4).

$\mathrm{Na}$ validatie van de monofasische actie-potentiaal-techniek voor de registratie van late nadepolarizaties tijdens ouabaine toediening, is de techniek gebruikt om de gevoeligheid van het hypertrofe hart voor getriggerde activiteit te bestuderen (hoofdstukken 5 en 6). Deze studies zijn gecombineerd met studies naar de veranderingen in electrofysiologie, functie en histologie van het hart ten gevolge van compleet atrioventriculair block (hoofdstukken 5, 6 en 7). Groepsvergelijkingen tussen honden met chronisch compleet AV-block (met hypertrofie) en honden met een normaal hartritme (zonder hypertrofie) toonden geen veranderingen in fibrose en capillair/myociet ratio. Dit suggereert een gelijkmatige toename in spiermassa, bindweefsel en bloedvaten (hoofdstuk 5). De honden met chronisch compleet atrioventriculair blok tonen geen lichamelijke symptomen van hartfalen. De normalisatie van plasma neurohormonen de afwezigheid van mRNA ANF expressie op weefselniveau en de verbeterde functie van het hart na 6 weken AV-blok bevestigen deze observatie (hoofdstukken 5,6 en 7).

Ondanks het feit dat de histologische, neurohumorale en functionele data aangeven dat het hypertrofe hart met compleet atrioventriculair blok adequaat is aangepast om de functie wan het hart te kunnen handhaven, is dit hart gevoeliger voor 1) het optreden van Torsade de Pointes ritmestoornissen (hoofdstuk 5) en 2) op late nadepolarizaties gebaseerde getriggerde ritmestoornissen (hoofdstuk 6). De verhoogde gevoeligheid voor Torsade de Pointes lijkt hierbij gerelateerd aan de niet-uniforme verlenging van de actie-potentiaal duur en een toegenomen gevoeligheid voor $d$-sotalol, terwijl het ontstaan van op late nadepolarizaies gebaseerde ritmestoornissen verband lijken te houden met 
een verhoogde contractiele staat van het hart. Tenslotte worden in hoofdstuk 8 de bevindingen uit deze studie besproken in relatie tot bevindingen uit de literatuur. 


\section{Dankwoord}

Inmiddels is het al meer dan 6 jaar geleden dat ik voor de eerste keer "mijn hoofd om de deur stak" bij de cardiologie in het enige echte Bio Medisch Centrum. Nog volstrekt onwetend van wat mij allemaal te wachten stond, vroeg ik Marc en Jet "wat zo'n student-assistentschap nu eigenlijk inhield". Natuurlijk had ik nooit kunnen voorspellen dat dit het begin zou zijn van dit proefschrift. Bijna aan het einde van dit boekje is het de hoogste tijd om het meest gelezen hoofdstuk te schrijven en om iedereen te bedanken die een bijdrage heeft geleverd aan dit resultaat.

Marc Vos, mijn begeleider en co-promotor, wil ik als eerste bedanken. Beste Marc, jouw dagelijkse begeleiding en ideeën vormen de basis van dit proefschrift. Door je onuitputtelijke inzet en positieve instelling, vind je zelfs in de meest uitzichtloze situaties een lichtpuntje. De afgelopen jaren werden gekenmerkt door talloze discussies, die overigens niet alleen beperkt bleven tot wetenschappelijke onderwerpen. Ons, maar met name jouw, eigenwijze gedrag zullen mij altijd bijblijven. Ook mijn eerste kennismaking met de United States, inclusief Basketball (uitverkocht), Football ("sportsbar"), Bier (weinig), Nachos (veel) en gevaarlijke Pizza (Atlanta 1994) zal ik nooit vergeten. Ondanks je omscholings-pogingen zal ik toch een echte sport-analfabeet blijven.

Mijn promotor, Prof. dr. Hein Wellens wil ik bedanken voor zijn belangstelling voor het onderzoek, de vruchtbare discussies en het kritisch lezen van de manuscripten, waarmee ik $u$ zelfs thuis mocht belagen. Uw enthousiasme en opbeurende woorden, waren een stimulans om verder te gaan. Tevens wil ik $\mathbf{u}$ bedanken voor de mogelijkheid te leren van uw enorme cardiologische kennis.

De leden van de beoordelingscommissie, Prof. dr. H.A.J. Struijker Boudier, Prof. dr. M.A. Allessie, Prof. dr. E. Carmeliet en Prof. dr. H.E.D.J. ter Keurs, wil ik bedanken voor het kritisch doorlezen van het manuscript en voor de bereidheid op de verdediging van mijn proefschrift aanwezig te zijn. Also, I would like to thank Prof. dr. M.R. R.osen for reviewing the manuscript and his willingness to be present at my thesis defense.

Waarschijnlijk ben ik een van de weinige promovendi, wie gesteund wordt door twee paranimfen èn één "associate-paranimf". Jet, samen hebben we vele uren in de O.K. doorgebracht. Afhankelijk van de "spanning" van het experiment werden naast dlata ook heel veel ècht belangrijke zaken besproken. Met een ontembare inzet heb je gewerkt aan 
de analyse van de data en de fraaie illustraties in dit proefschrift. I $k$ hoop dat we nog veel samen kunnen ondernemen.

Paul, jou wil ik niet alleen bedanken voor de vele discussie ower de resultaten van dit proefschrift, maar ook voor de culinaire estafette (knoflook is toch zo'n fijne smaakmaker), de handige huis-tips en de gezellige uitstapjes met als hoogtepunt "De Provence". Natuurlijk heeft Ilja bij dit alles een essentiële rol gespeeld. Paul en Jet, mede door jullie niet te stoppen, nachtelijke inzet (zelf had ik er lang het bijltje bij neer gegooid), is dit boekje toch nog op tijd bij de drukker gekomen. Ik ben erg blij dat jullie als mijn paranimfen aan mijn zijde willen staan.

Mijn "associate-paranimf", Dr. Simon Braat, wil ik bedanken voor je succesvolle inzet bij het verwerven van de broodnodige subsidies en voor het gebruik van zijn exclusieve computer-apparatuur.

Behalve Jet en Paul, wil ik de rest van de "experimentele cardiologie clan" bedanken: Karin Sipido voor haar commentaar op het manuscript, Cora voor jouw encyclopedie, Roel voor het scannen en de excell plaatjes, Jérôme voor je weerwoord, Ferenc voor je nimmer aflatende belangstelling en de vele liftjes naar huis, Henny voor het relativeren en Jurren voor de betere grappen. Marc Hermans, Marieke Schoenmakers en Mirella Molenschot zijn als student-assistent bij het onderzoek betrokken geweest. Tijdens de periode van deadlines hebben Marieke en Mirella fantastisch gewerkt om alles af te krijgen. Daarnaast is het ze gelukt om het ook nog gezellig te houden: hiervoor ben ik jullie zeer erkentelijk.

Samen met Jan Schreuder hebben we de gezellige en zeer steriele (op zijn Jan's) pressure-volume experimenten uitgevoerd. Voor verwerking en interpretatie van deze data was zijn steun onontbeerlijk. Ook Theo van der Nagel's hulp bij deze en andere experimenten heeft vele "kleine" problemen voorkomen. Ruud Kruger, ik weet dat je me niet meer gellooft, maar eigenlijk doe ik hele gemakkelijke experimenten. Bedankt voor je hulp. Jo Habets, ondanks het feit dat je mijn naam niet meer wist, wil ik je toch bedanken voor alles.

De Centrale Proefdier Voorzieningen onder leiding van Ton van den Boogaard wil ik bedanken voor het verzorgen van de honden. Huub Simons, jouw betrokkenheid bij het wel en wee van "onze" honden is in de laatste jaren alleen maar gegroeid. Hierdoor heb je jezelf onmisbaar gemaakt. De Instrumentele dienst van de Universiteit Maastricht, in 
het bijzonder Léon Dohmen, en de Audio-Visuele dienst van het Academisch Ziekenhuis, wil ik bedanken voor hun hulp.

Het secretariaat van de vakgroep cardiologie wil ik bedanken voor hun belangstelling en medeleven bij de afronding van dit proefschrift. Kleine Vivian en Viviane voor hun hulp bij het typen van brieven en het vinden van adressen. Birgit voor de "gouden promotietips". De "automatiserings- en informatievoorziening Cardiologie", alias Rob en Berto voor jullie hulp bij computerproblemen.

In de vrije tijd is het belangrijk gesteund te worden door een harde kern van echte vrienden. Garcia, Nicole en Simone, bedankt voor jullie steun en begrip, de lol en de nieuwe energie. Paul en Ilja (nogmaals) voor de vacantie, de drank, het eten en het gelach, Marc en Maya voor de bijvoeding, Lucas voor het voorbeeld, Esther, Bart, Carolijn, Roelie en Janice voor al het andere en tenslotte het Tennis Team van Captain Broeders voor de lichaamsbeweging en mijn afwezigheid.

Mijn kleine zusjes Harmke en Judith (ook al zijn jullie inmiddels al wat groter) hebben altijd geluisterd naar mijn verhalen, ook al raakte deze soms kant nog wal. Harmke, ik vind het fantastisch dat je de opmaak van dit boekje hebt verzorgd, het ziet er zeer fraai uit. Onze hot-line kan nu voor dit doel worden opgeheven. Claudia Volders wil ik uiteraard bedanken voor het verzorgen van de illustratie op de omslag. Hopelijk vind jij deze net zo mooi als ik!

Mijn ouders kan ik in deze paar regels onmogelijk bedanken voor alles wat ze voor me hebben gedaan en nog steeds doen. Papa en mama, jullie hebben me van kinds af aan gesteund bij alles wat ik deed. Ook bij mijn studie stimuleerden jullie mij een eigen weg te vinden. De afronding van dit proefschrift is mede aan jullie te danken.

Liefste Maurits, na de drukke tijd van jouw promotie zijn we bijna ongemerkt in de drukte van mijn promotie gekomen. Ondanks het feit dat ik jou en ons nieuwe huis volledig heb verwaarloosd, heb je me onvoorwaardelijk gesteund en zo veel mogelijk geholpen. Nu kunnen we ons samen storten op het volgende project! 


\section{Publications}

\section{Articles:}

M.A. Vos, A.P.M. Gorgels, G.C. Lipcsei, S.H.M. de Groot, H.D.M. Leunissen, H.J.J. Wellens. Mechanism specific anti-arrhythmic effects of the potassium channel activator levcromakalim against repolarization-dependent tachycardias. $J$ Cardiovasc Electrophysiol 1994; 5: 731-742.

S.H.M. de Groot, M.A. Vos, H.D.M. Leunissen, A.P.M. Gorgels, H.J.J. Wellens. Combining monophasic action potential recordings with pacing demonstrates delayed afterdepolarizations and triggered arrhythmias in the intact heart; the value of the diastolic slope. Circulation 1995; 92: 2697-2704.

M.A. Vos, S.H.M. de Groot, S.C. Verduyn, J. van der Zande, H.D.M. Leunissen, J. Cleutjens, M. van Bilsen, M. Daemen, J. Schreuder, M.A. Allessie, H.J.J. Wellens. Enhanced susceptibility for acquired Torsade de Pointes arrhythmias in the dog with chronic, complete AV-block is related to cardiac hypertrophy and electrical remodeling. Accepted for publication in Circulation

\section{Abstracts:}

M. Vos, A. Gorgels, H. Leunissen, G. Lipcsei, S. de Groot, H. Wellens. Specific anti-arrhythmic effects of the $\mathrm{I}_{\mathrm{k}}$-activator Lemakalim in vivo. Eur Heart $J 1992 ; 13 ; 61$.

M.A. Vos, M. de Groot, G.E. Lipcsei, J.D. Leunissen, A.P. Gorgels. Lemakalim demonstrates mechanism specific anti-arrhythmic properties without proarrhythmogenity. Circulation 1992; 86: I-560.

S. Marieke de Groot, Marc A. Vos, Anton P.M. Gorgels, Jet D. Leunissen, Bert J. van der Steld, Hein J.J. Wellens. The Diastolic Slope as a Parameter to Demonstrate Delayed Afterdepolarizations and Triggered Arrhythmias in Vivo: Validation with Lidocaine. $J$ Am Coll Card 1994; 466A. 
Marieke de Groot, Marc Vos, Anton Gorgels, Jet Leunissen, Hein Wellens. Delayed Afterdepolarization Dependent Ventricular Tachycardia; Quantification of Behavior Prior to Termination. PACE 1994; 17: II-121.

S. de Groot, M. Vos, A. Gorgels, H. Leunissen, H. Wellens. Delayed afterdepolarization dependent ventricular tachycardia; prediction of termination by the diastolic slope. Eur Heart J 1994; 15: P1058.

Marieke de Groot, Marc Vos, Marc Hermans, Jet Leunissen, Anton Gorgels, Hein Wellens. The height of the diastolic slope of the monophasic action potential is related to duration of pacing and determines the arrhythmic response postpacing. PACE 1995; 18: 344 .

M.A. Vos, S.H.M. de Groot, J. van der Zande, S.C. Verduyn, H.J.J Wellens. Electrophysiological changes observed after creation of AV-block in the dog. PACE 1995; 18: P1094 (part II).

S.C. Verduyn, M.A. Vos, J. van der Zande, S.H.M.A. de Groot, H.J.J. Wellens. Biventricular hypertrophy increases the arrhythmogenic effects of D-sotalol in dogs. Pftügers Archiv 1995; 430: R163.

S. Cora Verduyn, Marc A. Vos, Jolanda van der Zande, Marieke de Groot, Maurits A. Allessie, Hein J. Wellens. Biventricular hypertrophy facilitates the occurence of acquired torsade de pointes arrhythmias in dogs. Circulation 1995; 92: 1504-I505.

Verduyn SC, Vos MA, van der Zande J, de Groot SHMA, Allessie MA, Wellens HJJ. Biventricular Hypertrophy induced prolongation Facilitates Acquired Torsade de Pointes Arrhythmias in Dogs. Cardiologie 1995; 2: 444.

Marieke de Groot, Marc Vos, Jet Leunissen, Hein Wellens. The effect of ryanodine determines the rate of delayed afterdepolarization dependent ventricular tachycardia. PACE 1996; 16: 604 . 
S.H.M. de Groot, M.A. Vos, H.D.M. Leunissen, H.J.J. Wellens. The behavior of ouabain induced delayed afterdepolarization dependent ventricular tachycardia is related to cardiac function. Pflügers Archiv (Eur J Physiol) 1996; 432: R3.

M.A. Vos, J. van der Zande, S.C. Verduyn, S.H.M. de Groot, A. Kulcsár, J. Cleutjens, M. van Bilsen, M. Daemen, H.J.J. Wellens. Physiologic hypertrophy after creation of complete atrioventricular block. Eur Heart J 1996: 17: 549.

Groot de M, Vos M, Muller A, Hardeveld van C, Zuidwijk M, Leunissen J, Verduyn C, Wellens $\mathrm{H}$. Compensated biventricular hypertophy facilitates the induction of both early and delayed afterdepolarizations. Cardiologie 1996: 3; 375.

M. de Groot, M.A. Vos, A. Muller, C. van Hardeveld, J. Leunissen, M. Schoenmakers, M. Zuidwijk, C. Verduyn, H.J.J. Wellens. Increased susceptibility for triggered arrhythmias in biventricular hypertrophy due to complete atrioventricular block. Heart failure: $1997 ; 349$.

M.C.E.F. Wijffels, R. Dorland, C.J.H.J. Kirchhof, C. Garrat, S.H.M.A. de Groot, M.A. Allessie. Mechanisms of arrhythmogenesis: Electrical remodeling in atrial fibrillation. $J$ Mol Cell Cardiol 1997: 29; A38.

S.H.M. de Groot, M.A. Vos, M. Schoenmakers, J.D. Leunissen, H.J.J. Wellens. Adaptations in contractile function at chronic complete AV-block make the heart more susceptible to triggered arrhythmias. Circulation 1997: 96; 1239-1240

L.M. Rodriguez, C. Timmermans, D. Donker, J. Smeets, M. de Groot, H.J.J. Wellens. Evidence of atrial remodeling in patients with chronic atrial fibrillation: Observations following internal cardioversion. $J$ Am Coll Cardiol 1998 in press 


\section{Curriculum Vitae}

\section{Marieke de Groot}

10 november 1968 1987

1988-1992

$1991-1992$

$1992-1995$

1 februari 1998
Geboren te Boxmeer.

V.W.O. diploma aan het Kruisheren Kollege te Uden

Doctoraalexamen Geneeskunde, Rijksuniversiteit Limburg, Maastricht.

Student-assistentschap bij de vakgroep Cardiologie, Academisch Ziekenhuis Maastricht, Maastricht. Vervolg studie geneeskunde voor artsexamen gecombineerd met promotie onderzoek, artsexamen behaald in september 1995 . Aanvang opleiding tot cardioloog 